
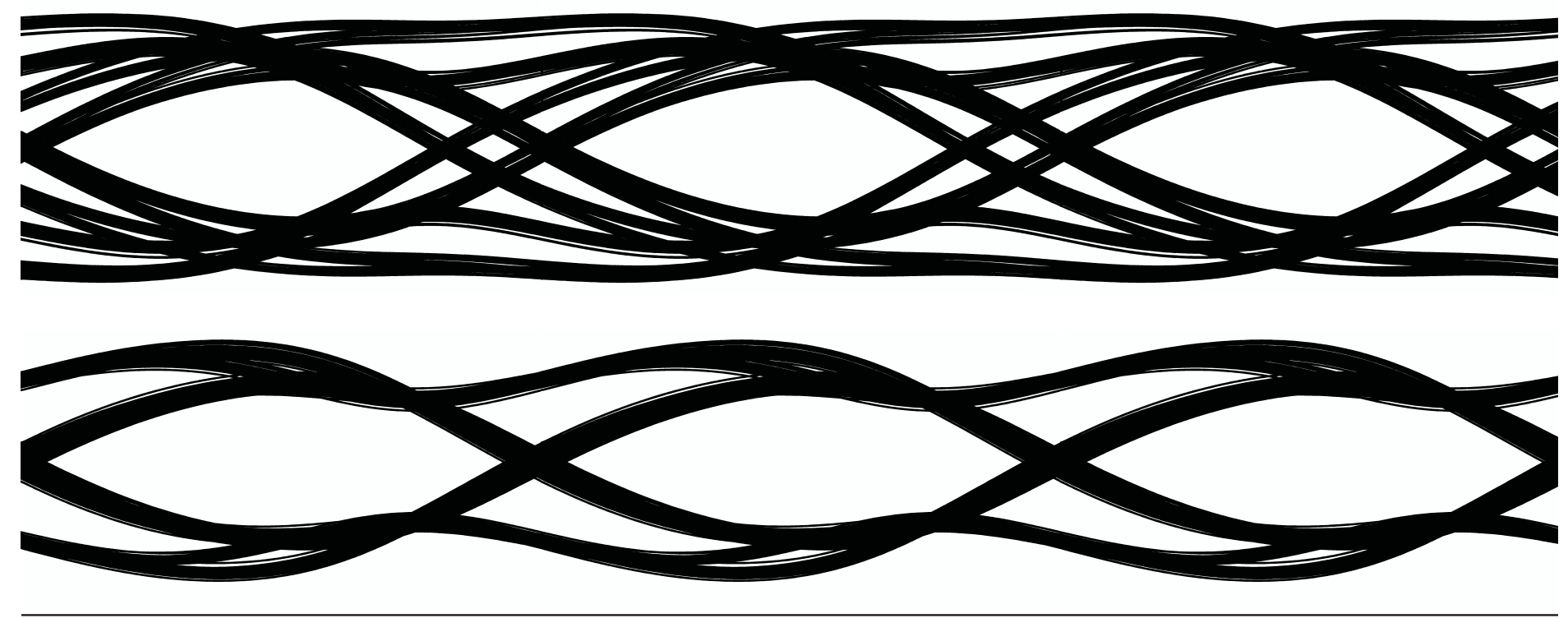

\title{
Wireline Equalization using Pulse-Width Modulation
}

\section{J.H.R. Schrader}

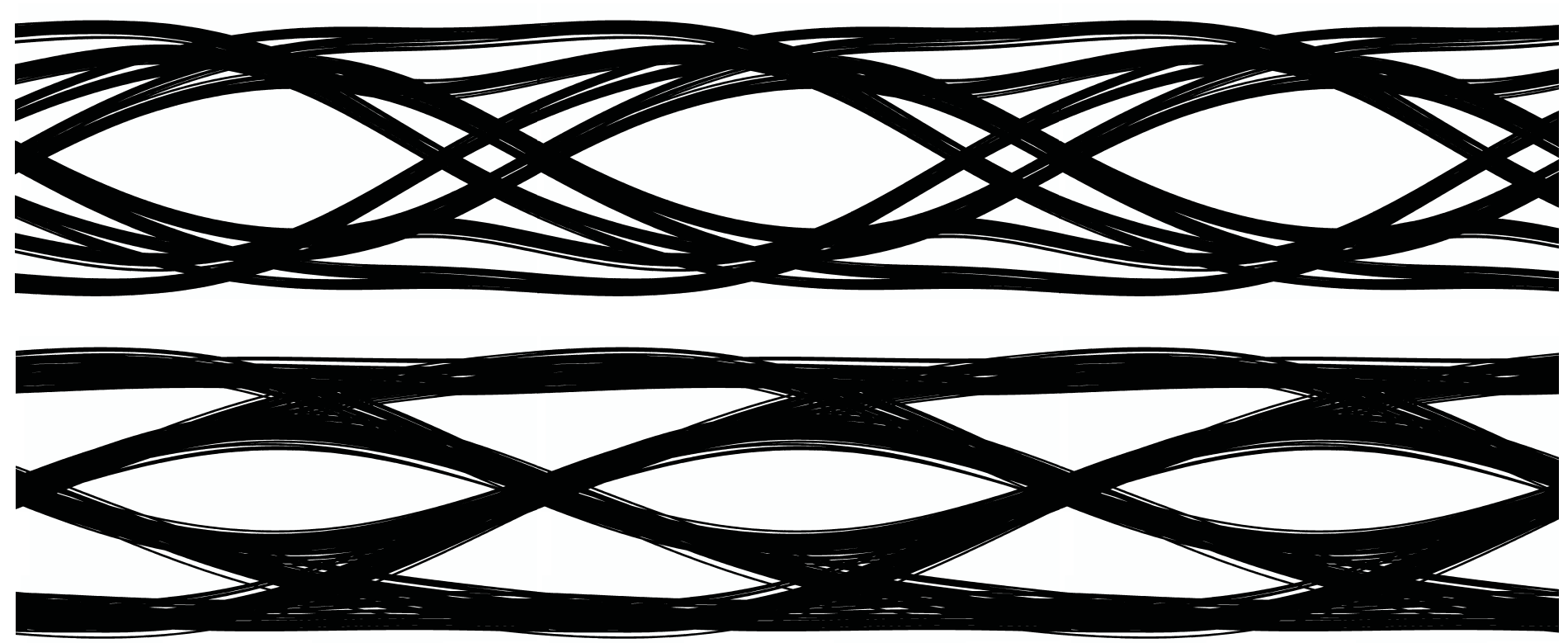





\section{Wireline Equalization using Pulse-Width Modulation}

Jan-Rutger Schrader 
Samenstelling promotiecommissie:

Voorzitter:

prof.dr.ir. A.J. Mouthaan

Universiteit Twente

Secretaris:

prof.dr.ir. A.J. Mouthaan

Universiteit Twente

Promotor:

prof.dr.ir. B. Nauta

Universiteit Twente

Assistent-promotor:

dr.ing. E.A.M. Klumperink Universiteit Twente

Referenten:

dr. J.L. Visschers

NIKHEF Amsterdam

dr. M. Kuijk

VU Brussel

Leden:

prof.dr.ir. W.C. van Etten

Universiteit Twente

prof.ir. A.J.M. van Tuijl

Universiteit Twente

prof.dr.ir. A.H.M. van Roermund

TU Eindhoven

CTIT Ph.D. thesis Series No. 07-104

Centre for Telematics and Information Technology (CTIT)

Contr for rolematics and
Intromation technology P.O. Box 217 - 7500 AE Enschede - The Netherlands

ISSN 1381-3617

This work is part of the research programme of the 'Stichting voor Fundamenteel Onderzoek der Materie (FOM)', which is financially supported by the 'Nederlandse Organisatie voor Wetenschappelijk Onderzoek (NWO)'.

(C) 2007 J.H.R. Schrader (http://www.schrader-ic.com)

Cover design: Tineke Schrader-Florijn

Printed by Gildeprint

ISBN 978-90-365-2567-1 
WIRELINE EQUALIZATION USING PULSE-WIDTH MODULATION

\section{PROEFSCHRIFT}

ter verkrijging van

de graad van doctor aan de Universiteit Twente,

op gezag van de rector magnificus, prof.dr. W.H.M. Zijm,

volgens besluit van het College voor Promoties

in het openbaar te verdedigen

op donderdag 11 oktober 2007 om 16.45 uur

door

Johan Hendrik Rutger Schrader

geboren op 27 september 1976

te Enkhuizen 
Dit proefschrift is goedgekeurd door:

de promotor prof.dr.ir. B. Nauta

de assistent-promotor dr.ing. E.A.M. Klumperink 
"Knowledge is a matter of piling up facts; wisdom lies in their simplification."

--Martin H. Fischer (1879-1962)

Aan mijn familie 



\section{Table of contents}

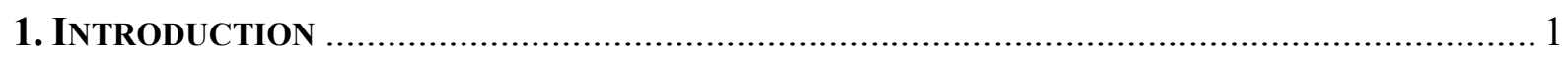

1.1. High speed data-communication over wires ................................................... 1

1.2. Types of copper interconnects and their behavior in the frequency domain .......... 3

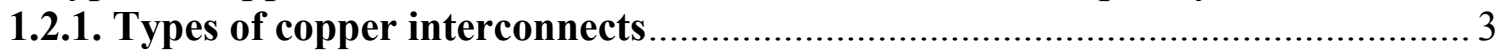

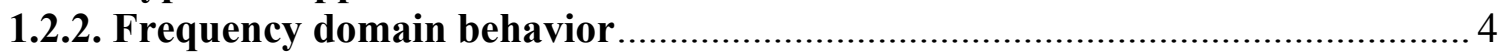

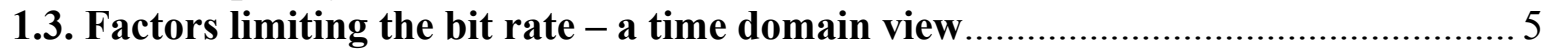

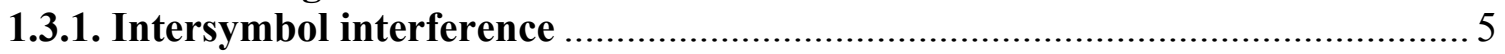

1.3.2. Reflections

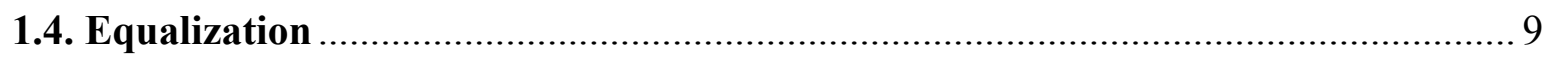

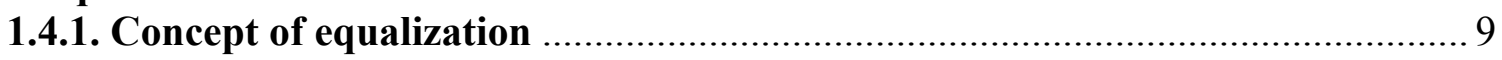

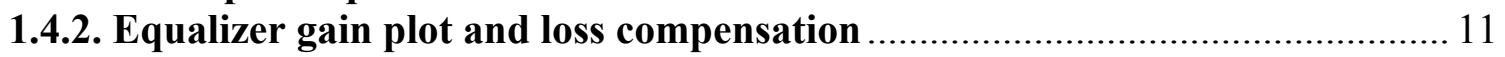

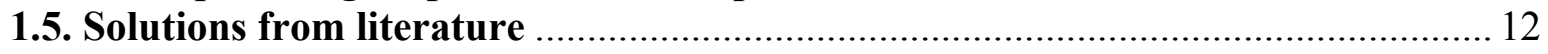

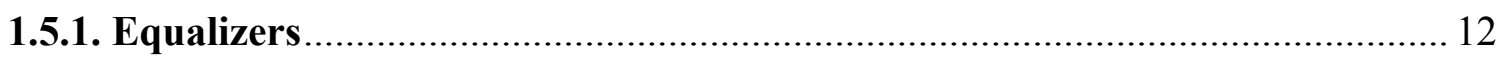

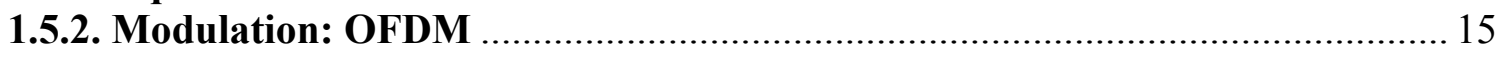

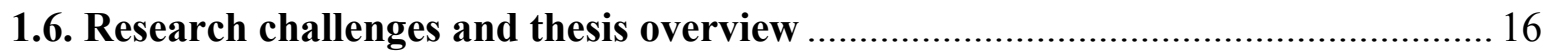

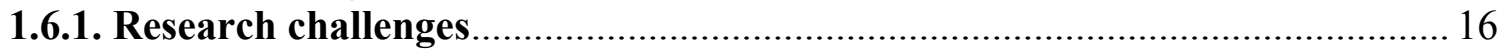

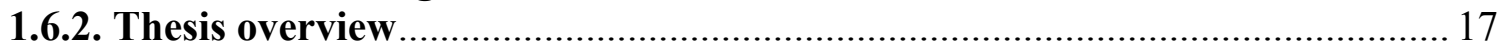

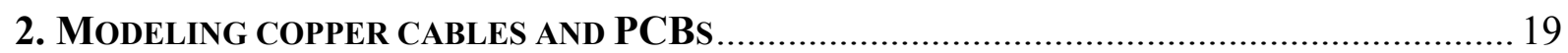

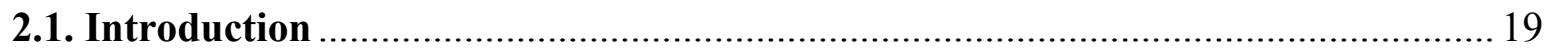

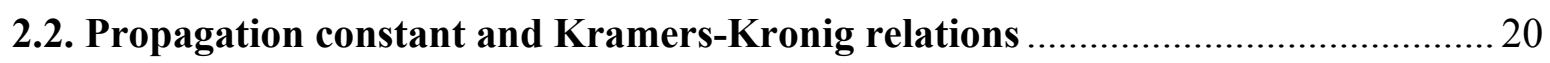

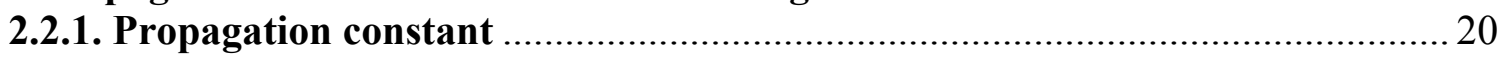

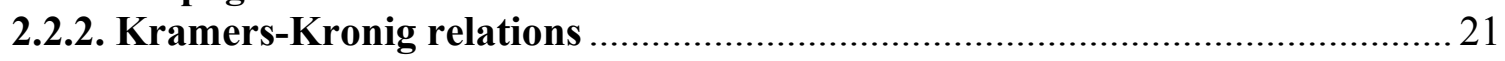

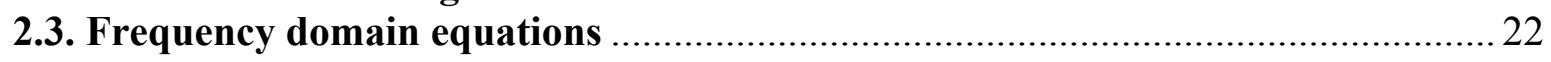

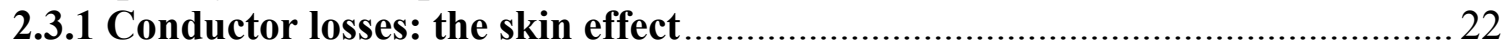

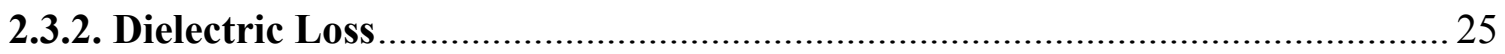

2.3.3. Isolated magnitude transfer function for skin loss and dielectric loss ........... 28

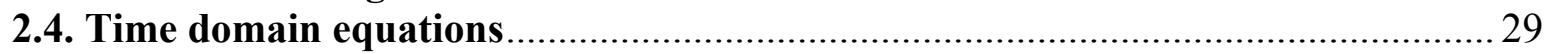

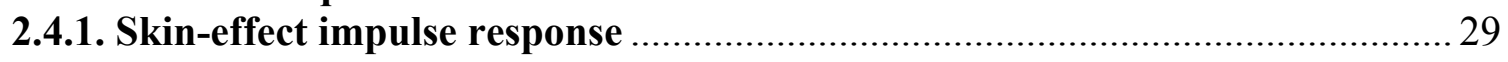

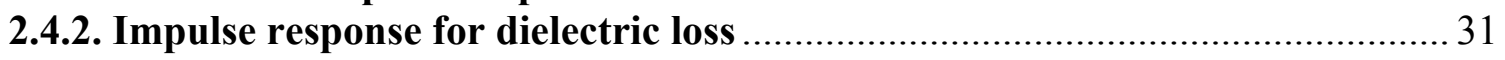

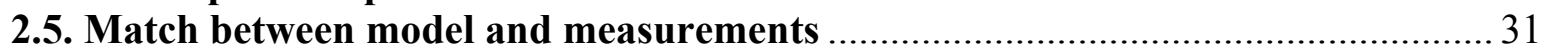

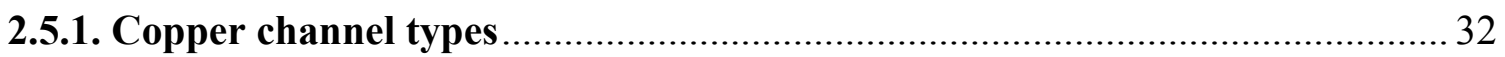

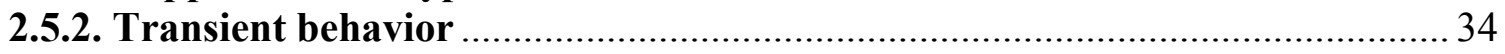

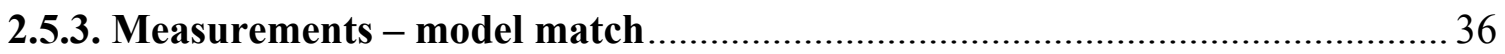

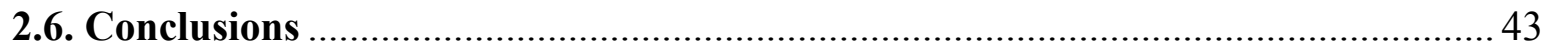

3. EFFECT OF TIMING NON-IDEALITIES ON AN ANALOG MULTICARRIER SYSTEM ............... 45

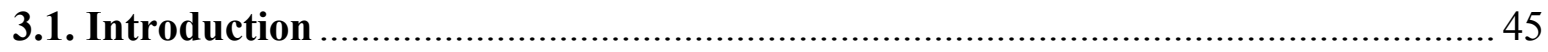

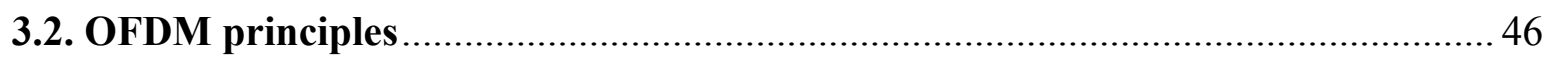

3.3. Practical proposal for 'analog OFDM' system architecture ............................... 46

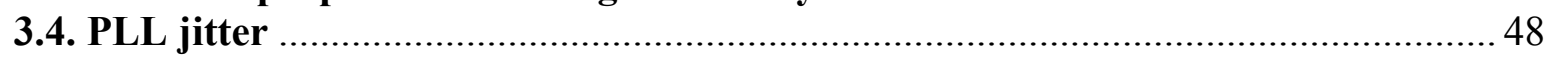

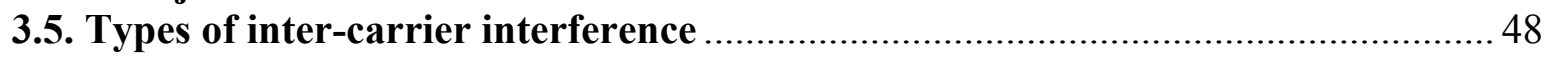

3.6. Impact of jitter on crosstalk between in-phase and quadrature signal at a given frequency 


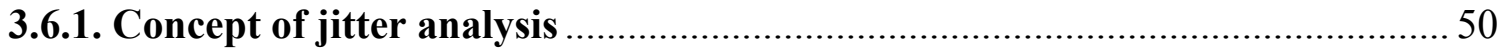

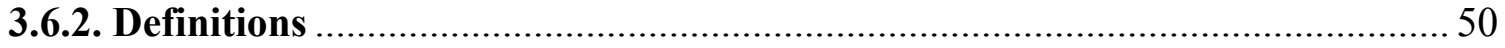

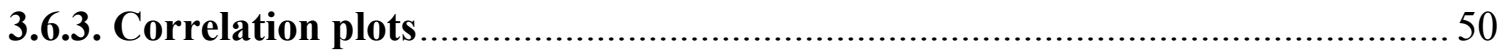

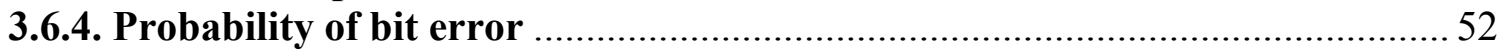

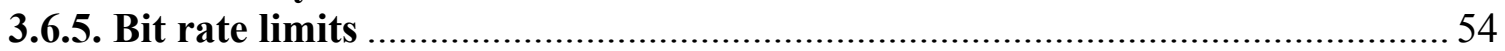

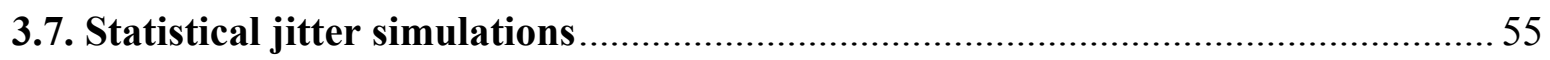

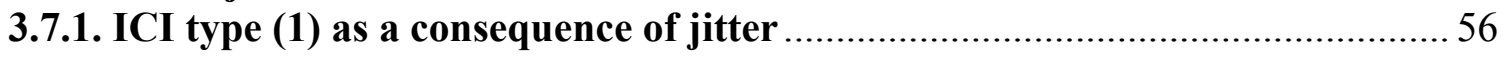

3.7.2. Impact of duty-cycle variations on carrier orthogonality ..............................5

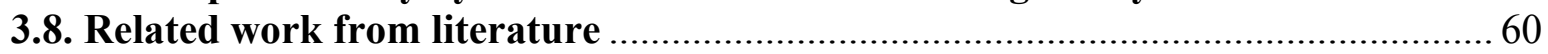

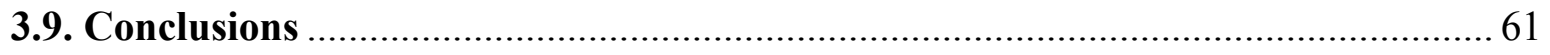

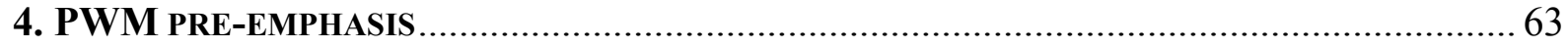

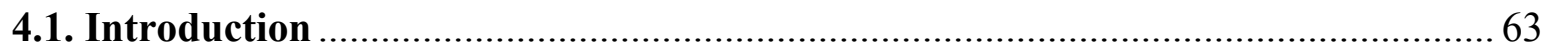

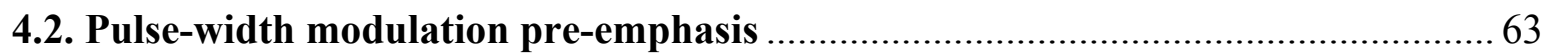

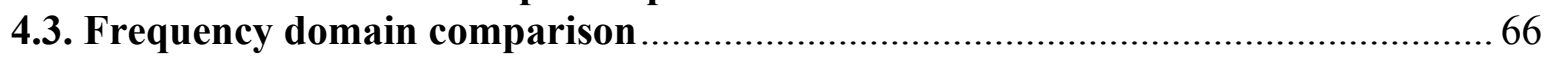

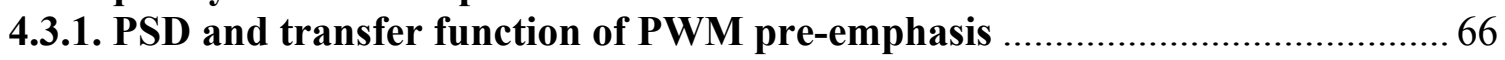

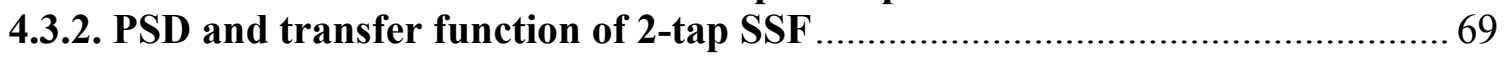

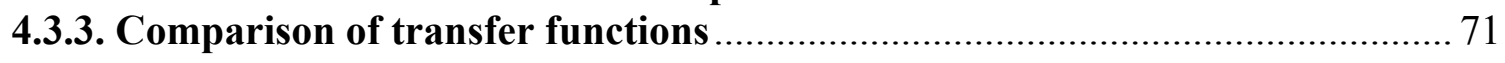

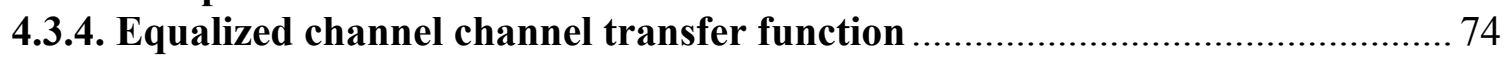

4.3.5. Filter DC behavior: move power to higher frequencies ................................... 75

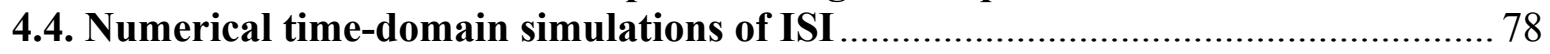

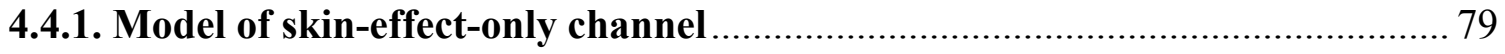

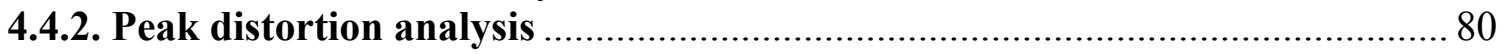

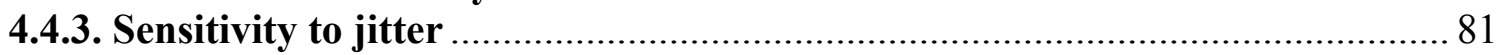

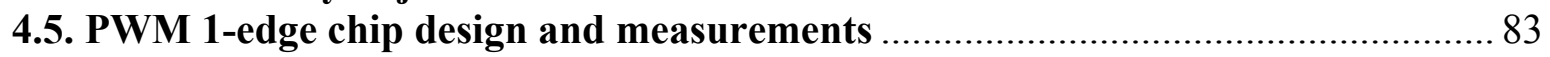

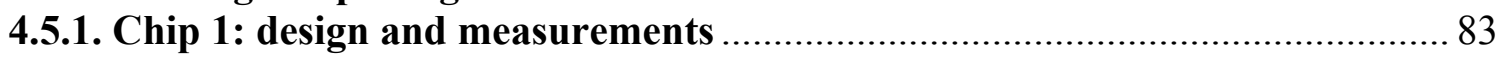

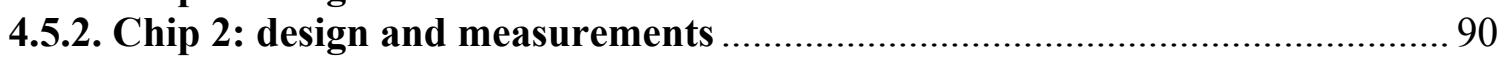

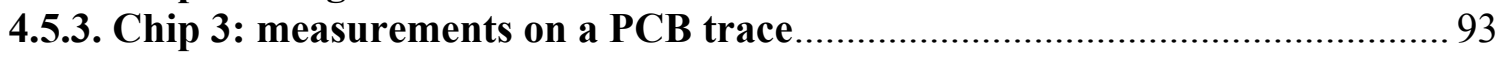

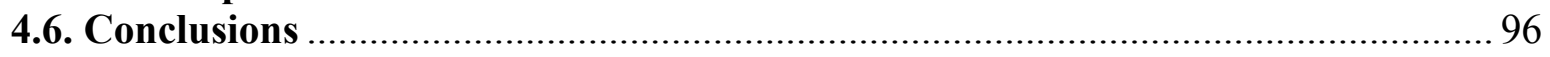

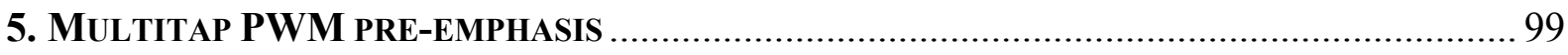

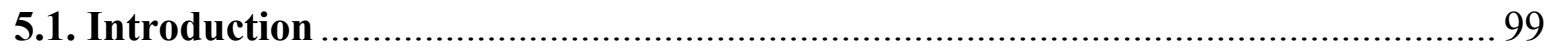

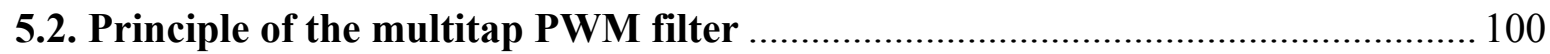

5.2.1. Same-area approach to translate FIR filter to PWM filter........................... 100

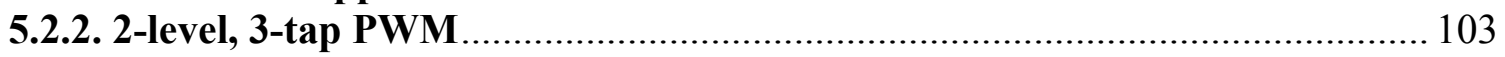

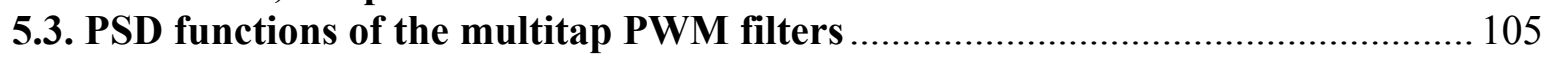

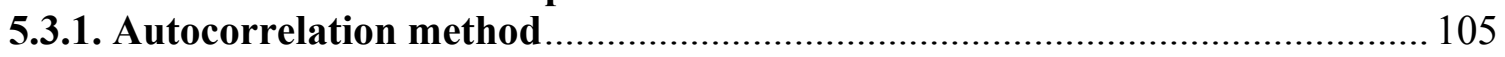

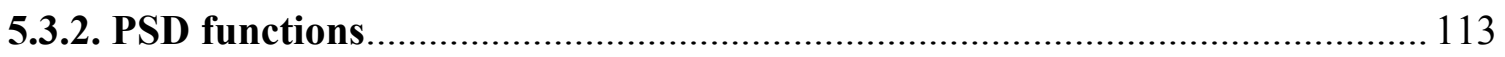

5.4. Reducing the number of transitions in the 2PWM scheme ................................ 115

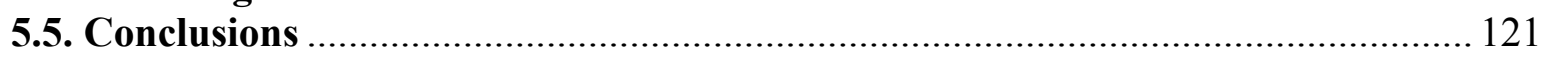

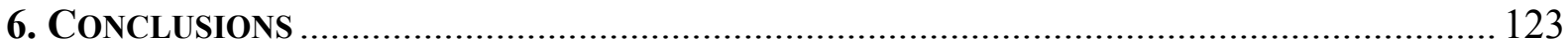

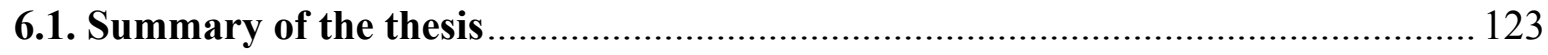

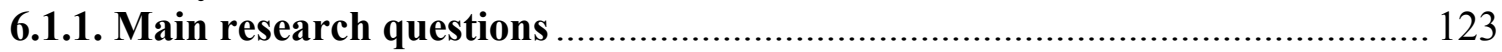

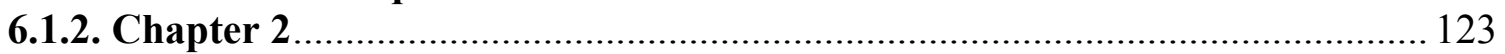

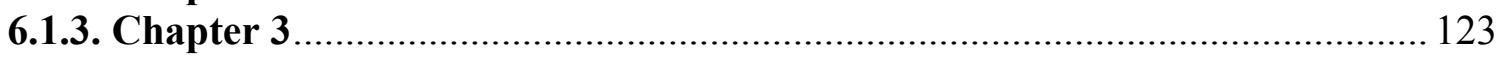

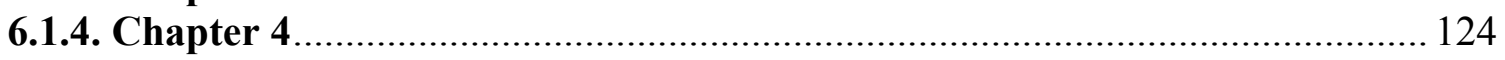

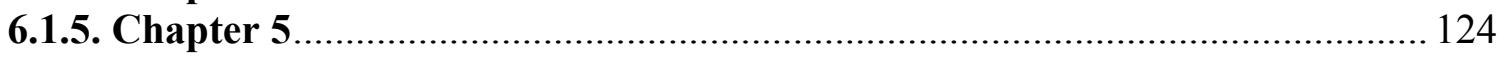

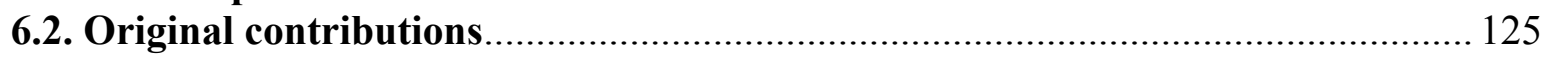

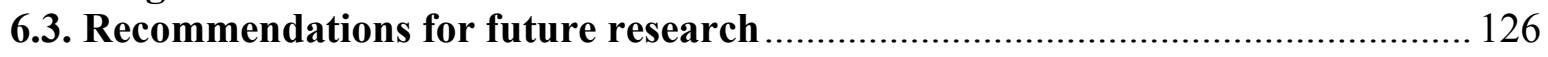


APPENDIX A. DIFFUSION EQUATION FOR SKIN EFFECT

APPENDIX B. JITTER DURING THE INTEGRATION PERIOD 129

APPENDIX C. TABLES FOR AUTOCORRELATION CALCULATIONS..

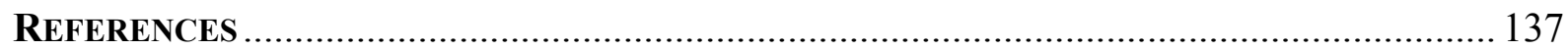

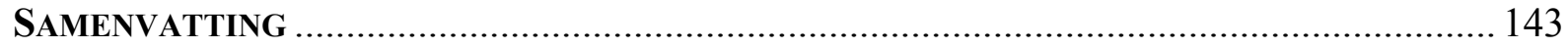

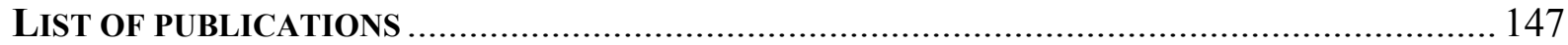

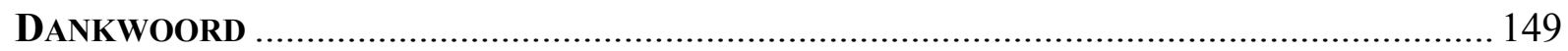

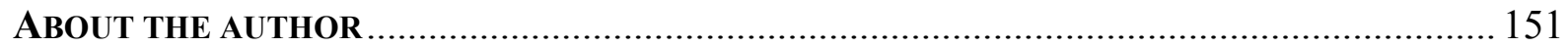





\section{Chapter 1}

\section{Introduction}

\subsection{High speed data-communication over wires}

Information and communication technologies are becoming ever more pervasive in our society. The growth rate of the Internet is exponential, with traffic doubling every year [Odlyzko]. There is a wealth of devices available that can be connected to a network or to each other.

These information and communication technologies are largely based on electronics, storing, processing and transmitting information in the form of bits (digital information units that can have a value of ' 1 ' or ' 0 ') and bytes (=8 bits). After the invention of the transistor, and subsequently the integrated circuit (IC), semiconductor technology also started to follow an exponential growth curve, known as 'Moore's law'. As is well known, IC scaling according to Moore's Law means that every new IC process will have more transistors per chip (and therefore smaller transistors) than the previous generation. Since the electrical capacitance of the individual transistors decreases, the switching speed increases, and so ever more bits per second can be processed in electronic circuits. This is the 'engine' driving the growth in the electronics, computer and Internet industries over recent decades.

At some point, bits and bytes need to be transferred from device to device using either cables (optical or electrical) or wireless connections. Although wireless connections can sometimes be more convenient, the maximum achievable bit rate that they offer is lower than for wired connections. This is because using wired connections, we are better able to focus and contain the electromagnetic field energy.

Several applications of wired connections which drive the need for ever higher communication speeds are given below.

In datacenters where information is stored and then distributed over the Internet, interconnections need to be made between servers, switches and routers. With the growth of the Internet, ever more information needs to be processed, and as a result it is essential to increase the bit rates on these interconnections.

Another demand for higher communication speeds is imposed by the home computer user, who (for example) wants to share a video with his family or friends over the Internet. He or she then needs to make a connection between the video camera and the PC to be able to upload the video. The quality of video available to home users is ever increasing (for example, in terms of resolution). Recently, HDTV quality has become available to the home user. The number of bits needed to encode an hour of video is much higher than with older video standards. The home computer user wants to wait seconds rather than minutes for the video to transfer to their PC. This means that the speed of such short interconnects between devices and the PC needs to be increased. 


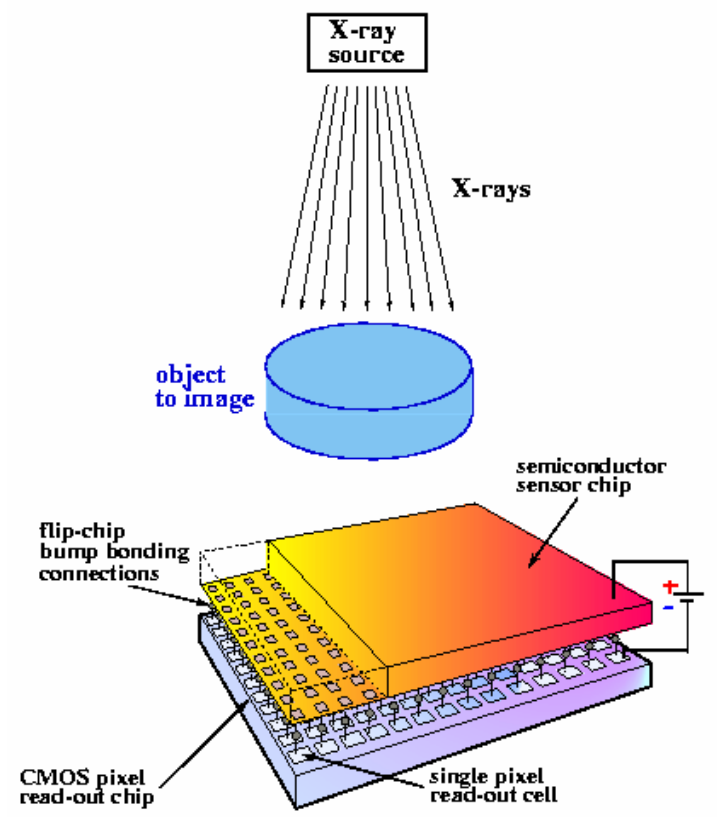

Fig 1. The Medipix2 setup [Llopart].

Inside the PC itself there are also many wired connections. For example, between the harddisk and the motherboard there is a high-speed interconnect. A current interconnect standard, Serial ATA (SATA), offers the advantages of thinner cables compared to old parallel ATA (PATA) interface. As a result, the ventilation though the PC casing is improved. SATA cables are also cheaper than cables for PATA. The decrease in cable thickness is achieved by serializing the data. This means that the number of channels is reduced (and the speed per channel is increased). Furthermore, the hard-disk read/write speeds are also increasing, and therefore the data rates over the interconnections between the hard-disk and the motherboard are also increasing.

Another interconnect inside the PC is that from the memory to the microprocessor. This connection is made using a printed circuit board (the motherboard). Processor speeds and bus clock rates are increasing so that ever more data can be processed per second. The speed at which this data needs to be fetched from and read into the memory is therefore also increasing.

This PhD project originally started by addressing the need for a high-speed interconnect that would connect the Medipix CMOS X-ray detector to a data storage system. This Medipix detector is a CMOS X-ray imaging chip [Llopart]. It is bump-bonded to a detector chip, such as high resistivity silicon, which is used to convert X-rays into hole-electron pairs. This is illustrated in Fig. 1. In future digital X-ray imaging systems, very high data rates will be needed. For some applications, e.g. protein crystallography at synchrotron beams, data rates in the order of gigabits per second are anticipated. The present trend for such systems is to move from a parallel data bus towards a high-speed serial link. Therefore, more bits per second need to be transmitted from the chip to a storage system.

All these wired interconnections can be formed by copper cables or glass fibers. Glass fiber offers a higher bandwidth-distance product, and therefore outperforms copper over long distances at high data rates. However, a high speed laser driver is more expensive than a straightforward connection of copper to an electronic circuit. Also the coupling of the light into a high speed fiber demands high precision. With the dominance of microchips as the 
primary means of signal processing, all the signals are produced in the electrical domain. Using copper interconnects offers the advantage that that no electro-optical conversion is needed before transmission. Copper can provide a transmission speed of the same order and at high reliability for interconnect lengths up to approximately $100 \mathrm{~m}$, provided that high quality cables are used.

Our area of study is limited to electronic circuits for high speed serial links over copper interconnects. In this thesis, we analyze, implement and test techniques to increase the data rate of communication over copper channels.

This Introduction is organized as follows. In section 1.2, an overview is provided of the different types of copper interconnects and of their behavior in the frequency domain. Next, in section 1.3, it is shown how the problem of intersymbol interference limits the achievable bit rate. In section 1.4 it is explained how an equalizer can be used to increase the bit rate. After that, in section 1.5, the advantages and disadvantages of specific equalizer implementations and modulation schemes from the literature are discussed. At the end of this chapter (section 1.6) the research challenges are listed and an overview of the thesis is provided.

\subsection{Types of copper interconnects and their behavior in the frequency domain}

In this section we discuss some of the most frequently used types of copper interconnections available (subssection 1.2.1), and look at their electrical behavior and capacity limit from a frequency domain point of view (subsection 1.2.2).

\subsubsection{Types of copper interconnects}

There are several types of copper cable and copper interconnect available for high speed links, as shown in Fig. 2. One type is the coaxial copper cable, which consists of a center conductor and an outer conductor, separated by an insulating dielectric, as shown in Fig. 2(a). This dielectric is typically polyethylene or Teflon, but can also be of another type, for example a low-loss dielectric. A plastic jacket is fitted to the outside. Another type, the twisted pair cable, consists of two twisted conductors, each surrounded by an insulating dielectric. Depending on the presence or absence of a metal shield, this cable is either called shielded twisted pair (STP) or unshielded twisted pair (UTP). The latter is commonly used for local area networks (LANs) in buildings. Again, a jacket is fitted to the outside, as shown in Fig. 2(b).

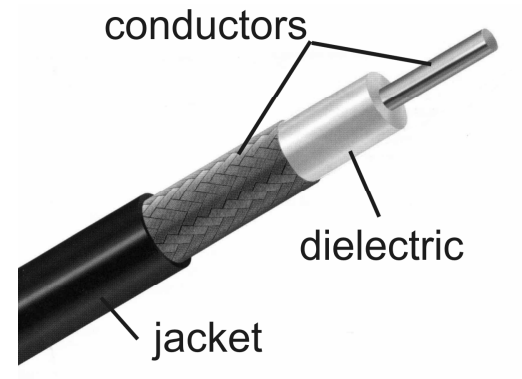

(a)

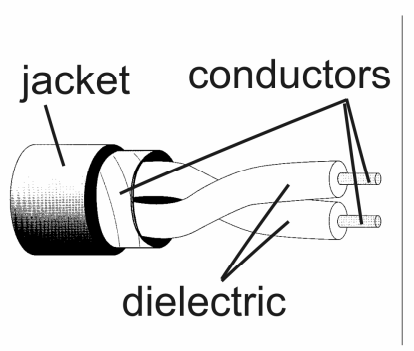

(b)

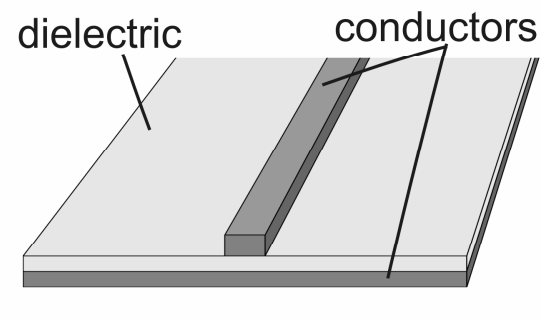

(c)

Fig. 2. Copper channels. (a) Coaxial copper cable. (b) Twisted pair copper cable.

(c) Microstrip on printed circuit board. 


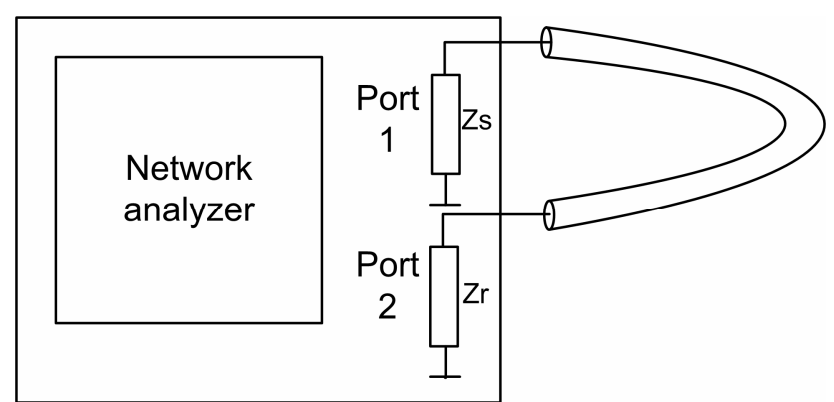

Fig. 3. Setup for loss measurement.

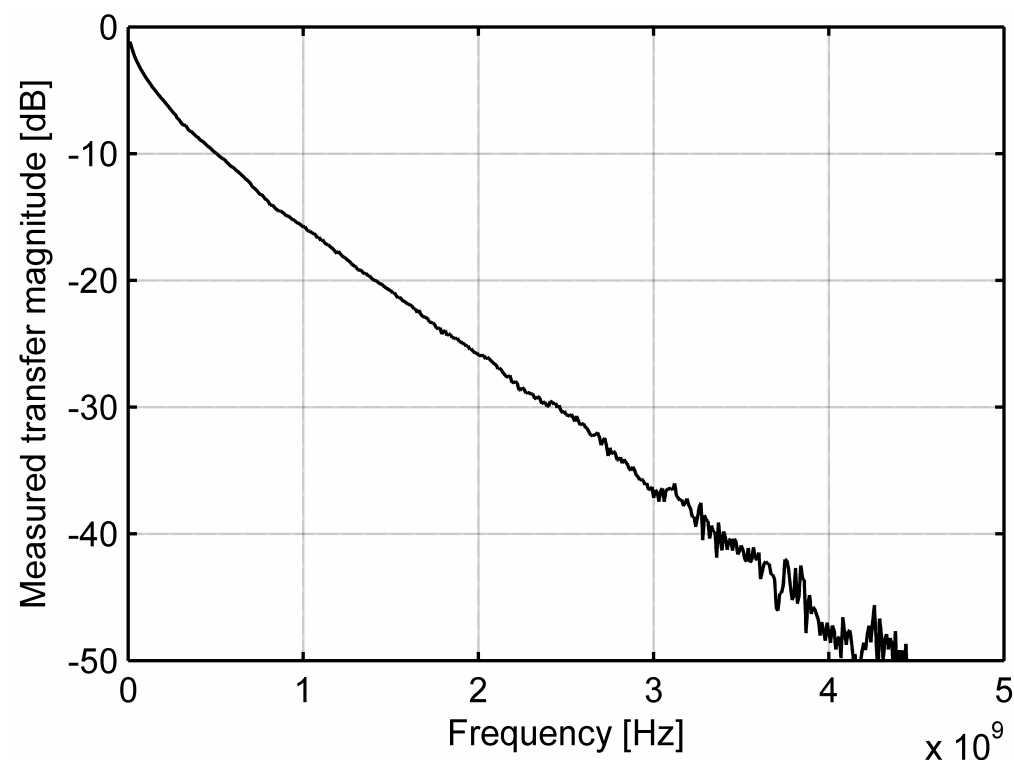

Fig. 4. Measured magnitude of transfer function of $25 \mathrm{~m} \mathrm{RG}-58 \mathrm{CU}$ coaxial cable.

A third type of copper interconnect, the printed circuit board (PCB), is commonly used to connect components. Examples are the motherboard inside a PC and the PCB inside a mobile phone. These boards are a sandwich of copper layers and dielectric layers. Fig. 2(c) shows a PCB with a single dielectric layer. The copper tracks can be etched using lithography. Several types of dielectric are available, the most common being the green-colored glass-epoxy FR4. More expensive, low-loss dielectrics are also available. See Fig. 2(c) for an illustration of a controlled-impedance microstrip over a groundplane.

\subsubsection{Frequency domain behavior}

We now look into the electrical behavior of these three copper channel types. How do they behave and what limits does this behavior impose on the achievable bit rate? Frequency domain measurements are shown, made using a network analyzer, to show the channel loss.

Clearly, practical conductors and dielectric materials fall short of the ideal. They exhibit undesirable attenuation ('loss'), because inside the conductor and dielectric, energy is converted from the electrical domain to other domains, for example to heat. They are also dispersive, which means that the signal delay between input and output varies with frequency.

To measure cable loss in the frequency domain, we use a network analyzer. Using the measurement setup shown in Fig. 3, we measure the channel loss (also known as magnitude of the transfer function, or the attenuation) as a function of the frequency for a $25 \mathrm{~m} \mathrm{RG}-58 \mathrm{CU}$ coaxial cable. Both ports of the network analyzer are terminated with the characteristic 


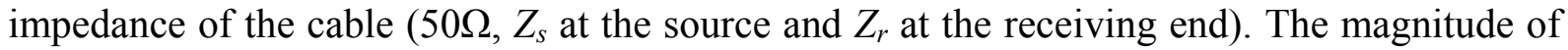
the transfer function $S_{21}$ is shown in Fig. 4. We plot the loss against a linear frequency axis.

\subsection{Factors limiting the bit rate - a time domain view}

Clearly the cable shows a loss that increases with frequency. To understand how this limits the achievable bit rate on copper channels, we change our point of view and look at the problem in the time domain. This section describes intersymbol interference caused by channel loss and dispersion (subsection 1.3.1) and reflections (subsection 1.3.2).

\subsubsection{Intersymbol interference}

The attenuation and dispersion effects can be seen in the time domain as intersymbol interference (ISI). In this subsection we illustrate ISI with the commonly known and widely used 2PAM modulation method for high-speed baseband transmission over copper channels [Dally], [Lee], [Kudoh].

The 2PAM modulation method simply uses one voltage to transmit a binary ' 1 ' and another voltage to transmit a binary ' 0 '. When these levels are opposite this is called polar NonReturn to Zero (polar NRZ). This modulation method represents the most straightforward, 'natural', off-chip output of the on-chip digital signals, after serialization. (Later, in Fig. 8, a 2PAM signal is shown).

We look at the ISI problem using the pulse response (1.3.1.1), the transient response (1.3.1.2) and the eye diagram (1.3.1.3). Important definitions of the Nyquist frequency and the bit error rate (BER), which are used throughout the thesis, are given along the way.

\subsubsection{Pulse response}

To understand ISI, we can look at the cable response to a single PAM pulse. The width of this pulse is equal to the bit length $T_{s}$ (which in turn is equal to the inverse of the bit rate $R$ ). In Fig. 6, our measurement setup is shown, consisting of a pattern/pulse generator, the channel,

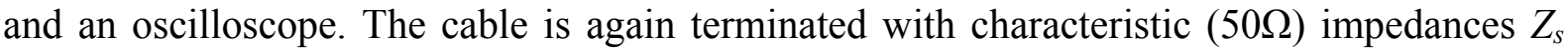
and $Z_{r}$ at the transmit and receive sides respectively.

The measured response of $25 \mathrm{~m}$ RG-58CU coaxial cable to a single $2 \mathrm{PAM}$ pulse of length $T_{s}=200 \mathrm{ps}$ (bit rate $5 \mathrm{~Gb} / \mathrm{s}$ ) is shown in Fig. 7. The cable output is time-shifted to compensate for the propagation delay. In the figure, the sampling point of the received pulse (the cursor) is indicated with a triangle and the sampling points of the next and previous symbols are indicated with circles. The circles with a nonzero value add to the ISI. A long tail on the right side can be seen, causing a high level of "post-cursor" ISI. There is also one "pre-cursor" ISI point. There is a clear asymmetry in the pulse response, which is indicative of dispersion. 


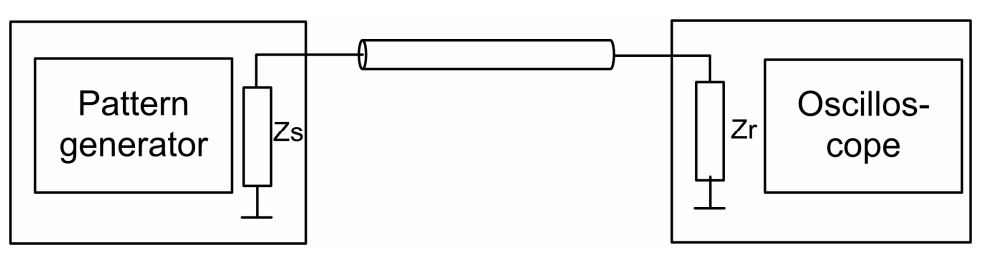

Fig. 6. Time domain measurement setup.

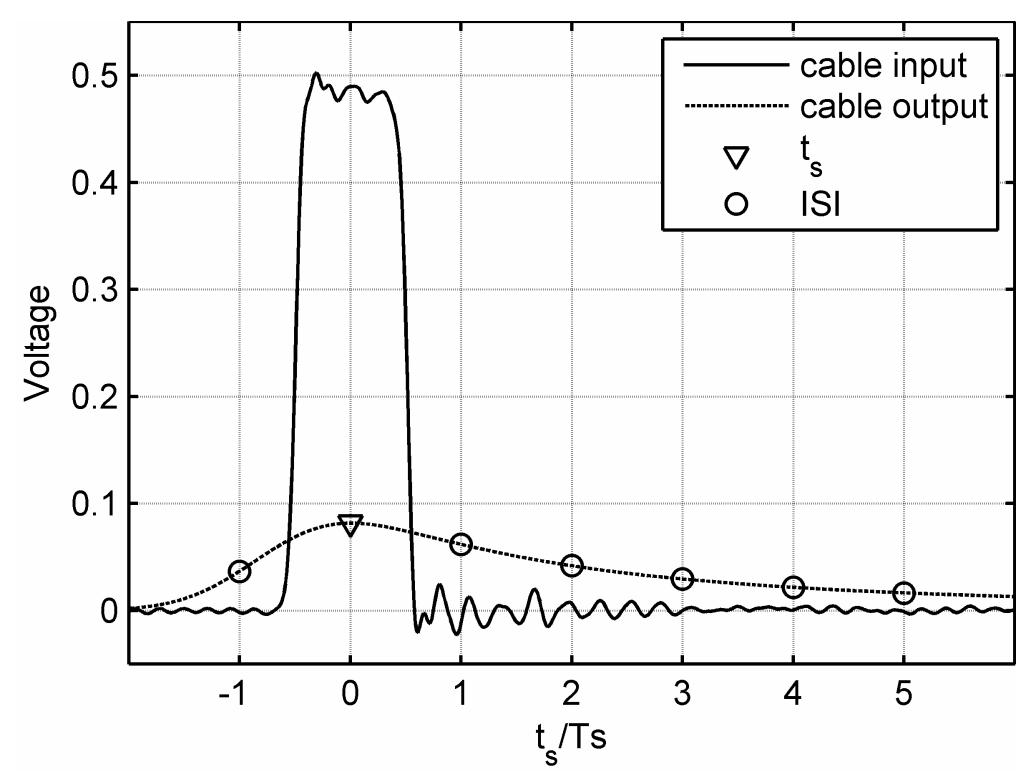

Fig. 7. Measured pulse response of 25m RG58CU (time shifted), and measured input pulse.

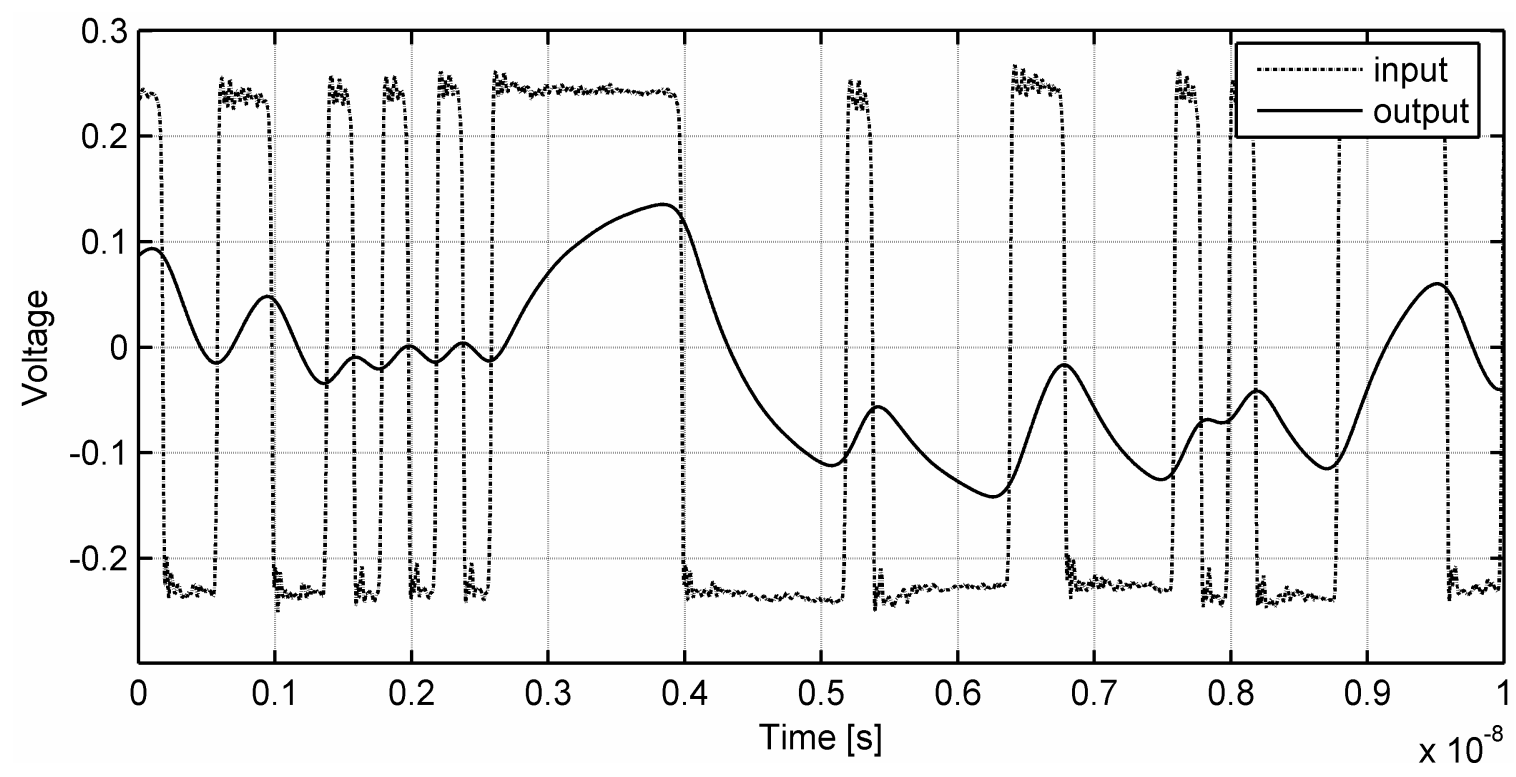

Fig. 8. Measured transient response of $25 \mathrm{~m} \mathrm{RG}-58 \mathrm{CU}$ coaxial cable. $5 \mathrm{~Gb} / \mathrm{s}$ data input and output. 


\subsubsection{Transient response}

When a string of 2PAM modulated bits is transmitted over the cable using the pattern generator, we obtain the result shown in Fig. 8. Clearly, the channel response is severely distorted by intersymbol interference (ISI). All the tails of the separate pulse responses are summed up. It is no longer possible to simply place a threshold at $0 \mathrm{~V}$ and decide whether the measured signal is above or below it. The channel has a low-pass transfer function, and therefore the high frequency (HF) components are attenuated more than the low frequency (LF) components. As a result, a 'baseline wandering' effect can be seen in Fig. 8.

\section{The Nyquist frequency}

The Nyquist frequency $f_{N}$ of a 2PAM signal is defined as half the bit rate. In our example, with a bit rate of $5 \mathrm{~Gb} / \mathrm{s}$, the Nyquist frequency is $2.5 \mathrm{GHz}$.

From the transient response shown in Fig. 8, we can roughly estimate the channel attenuation at $f_{N}$ by looking at the fastest transitions, e.g. from $1.5 \mathrm{~ns}$ to $2.5 \mathrm{~ns}$. Their amplitude is approximately $20 \mathrm{mV}$. Seeing that the amplitude of the cable input signal is approximately $0.5 \mathrm{~V}$, the channel attenuation at $f_{N}$ is $20 \mathrm{~m} / 0.5=0.04$, which corresponds to $28 \mathrm{~dB}$ loss. (Compare this with the frequency-domain measurement of $30 \mathrm{~dB}$ in Fig. 4.)

The significance of the Nyquist frequency for the design of equalizers is that we can use it to define a figure-of-merit for equalizers, as is shown later.

\subsubsection{Eye diagram}

The quality of the received signal can be accurately assessed using an eye diagram. An eye diagram is made by setting the oscilloscope $\mathrm{x}$-axis to one single bit time, so that all bits are plotted on top of each other. Effectively, the oscilloscope is now triggered by the bit clock. This results in an 'eye' shape, as illustrated in Fig. 9. Fig. 9(a) shows the input signal, with a clearly open eye, and Fig 9(b) shows the cable output, with a closed eye. The eye opening tells us a lot about the signal integrity. The eye opening and eye width can be used to estimate the voltage margin and timing margin respectively. This can be understood as follows. The receiver needs to sample each bit, so the sampling interval is $T_{s}$. The sampling position within the interval $\left(-T_{s} / 2, T_{s} / 2\right)$ is denoted as $t_{\text {sam. }}$. There is a certain allowance for deviation of the sampling position from $t_{\text {sam }}$, which is given by the timing margin. Furthermore, the receiver needs to decide whether the transmitted bit was either a one or a zero, by measuring its value relative to a threshold $v_{t h}$. The eye height tells us how large the voltage margin is. We need to have a certain margin because when noise (e.g. thermal noise) is added to the signal, there is a chance that the signal crosses the threshold and the bit will be detected wrongly, resulting in a bit error.

\section{Jitter}

Jitter (timing noise) in the receiver limits the symbol rate at which the receiver can reliably detect data. We can understand this using the eye diagram. When the sampling time $t_{\text {sam }}$ varies due to jitter, the timing margin in the eye diagram determines the allowable variation for $t_{\text {sam }}$ to prevent bit errors from occurring. 


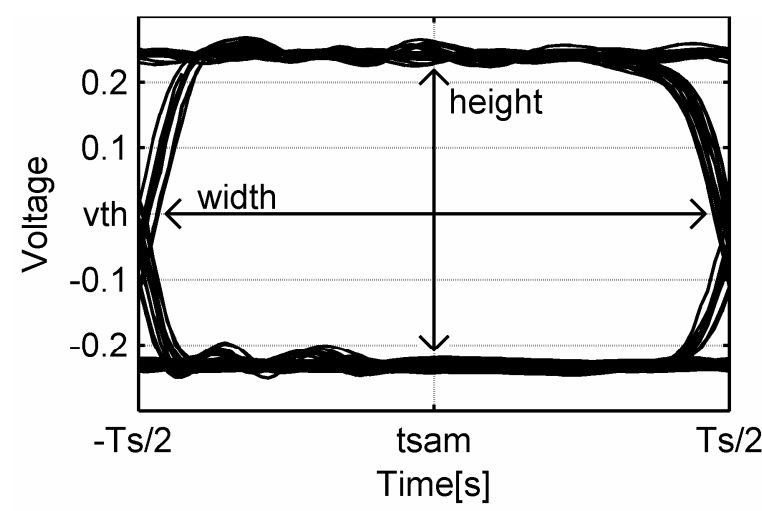

(a)

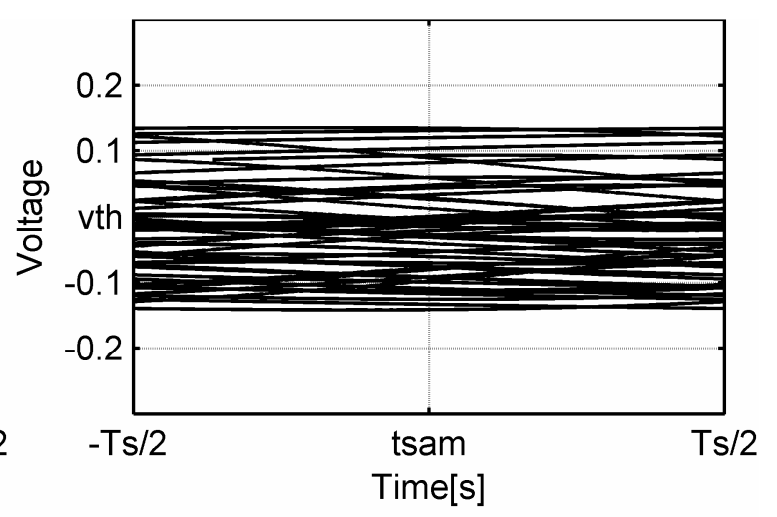

(b)

Fig. 9. Eye diagrams of measurements on $25 \mathrm{~m} \mathrm{RG}-58 \mathrm{CU}$ coaxial cable ( $\left.R=5 \mathrm{~Gb} / \mathrm{s}, T_{s}=200 \mathrm{ps}\right)$. (a) Cable input. (b) Cable output: eye completely closed by ISI.

The origin of this jitter depends on the system implementation. Often, the sampling time $t_{\text {sam }}$ is determined by a phase locked loop (PLL) via a clock and data recovery (CDR) loop. This PLL shows jitter, for instance because the thermal noise in the transistor channel translates into timing noise in the Voltage Controlled Oscillator (VCO). This happens for example when a noisy signal triggers a next stage in a ring oscillator or if the threshold of a comparator or logic gate is noisy due to thermal noise. The slew rate of the signal then determines the translation coefficient of the noise amplitude to timing noise [Abidi].

\section{Bit error rate}

When the receiver makes an incorrect decision about the received bit, a bit error occurs. This can be quantified using a statistical metric called the bit error rate (BER). This metric is defined as the ratio of the number of bits incorrectly received to the total number of received bits. For high-speed links, the target BER is typically in the order of $10^{-12}$ [Farjad-Rad].

\subsubsection{Reflections}

Another potential limit on the achievable bit rate comes from signal reflections.

At the connection of the transmission line to the circuit there are usually bondwires, and possibly a short section of another transmission line. At the boundaries between these lines there are often impedance discontinuities. Furthermore, connectors in the signal path might pose additional impedance discontinuities. When a signal traveling through a transmission line arrives at an impedance discontinuity, part of the signal will be reflected. This is undesirable, because reflections can distort the received bit pattern.

In the past, computer buses operating at low speeds would simply be designed to reflect $100 \%$ of the signal energy so as to double the received voltage. In $\mathrm{Gb} / \mathrm{s}$ serial links, this is not the usual approach. At $\mathrm{Gb} / \mathrm{s}$ speeds, the length of one bit traveling through a copper cable is only a few centimeters. For example, the length of one single bit in a $10 \mathrm{~Gb} / \mathrm{s} 2 \mathrm{PAM}$ signal traveling over a copper cable at a propagation speed of $2 / 3$ times the light-speed is only $2 \mathrm{~cm}$. This means that a $10 \mathrm{~m}$ long cable will typically have $\sim 500$ bits 'in transit'. 


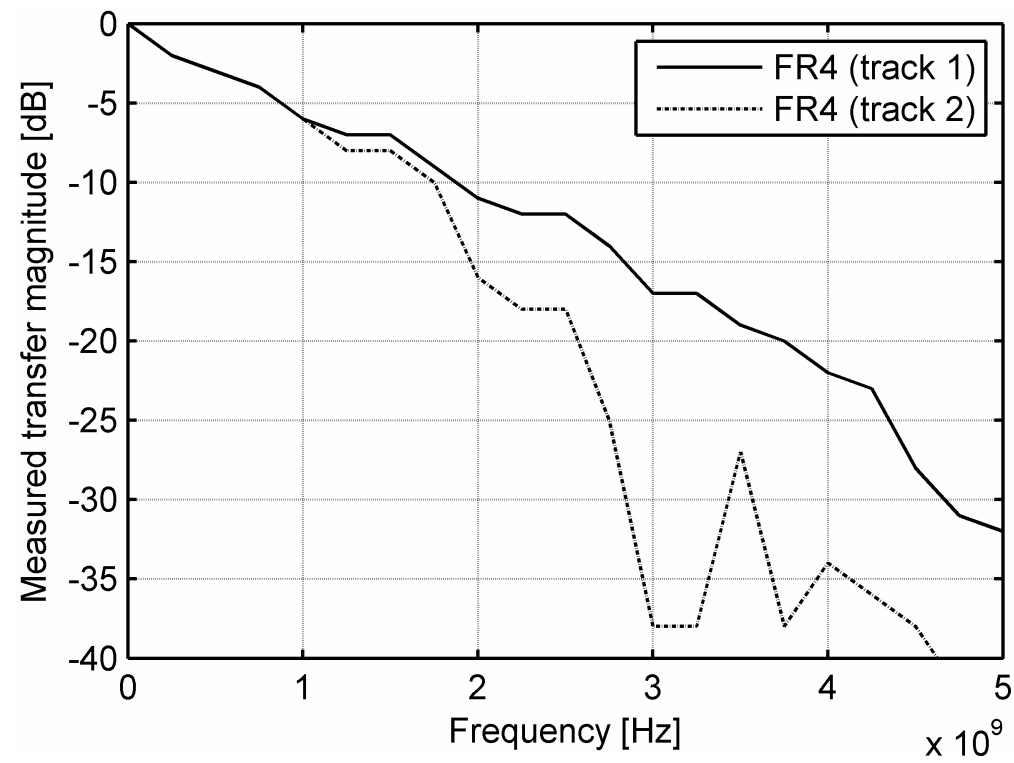

Fig. 10. Measured magnitude of transfer function of two 20" FR4 backplane tracks.

By using double termination, both at the transmitter and receiver end, the maximum signal integrity is achieved. This is called incident-wave signaling [Dally-2]. For good quality cables, reflections can be decreased to below $10 \%$, with well-controlled characteristic impedance of the cable and proper matching of the termination impedances - plus well designed connectors. Even so, a 10\% reflection added to the received signal will limit the effective SNR to $20 \mathrm{~dB}$.

For printed circuit boards, the problem of impedance discontinuities is harder to avoid than for cables. There are usually vias (connections through the dielectric) along the track which cause impedance discontinuities. Also connectors pose additional impedance discontinuities. This can lead to severe notches (spectral nulls) in the channel transfer function, as illustrated in Fig. 10.

\subsection{Equalization}

The previously discussed problems of ISI and reflections can be partially solved by using equalization. In this section, we explain the concept of equalization (subsection 1.4.1). A figure-of-merit (FoM) for equalizers is given next: the loss compensation (subsection 1.4.2).

\subsubsection{Concept of equalization}

Provided that the SNR is high enough, it is possible to reconstruct a PAM signal that is lowpass filtered by the channel. To achieve this, an equalizing filter is placed in series with the channel. This filter can be placed either at the transmitter side, where it is called a TX pre-/deemphasis filter, or at the receiver side, where the technique is called RX equalization. (Illustration in Fig. 11.)

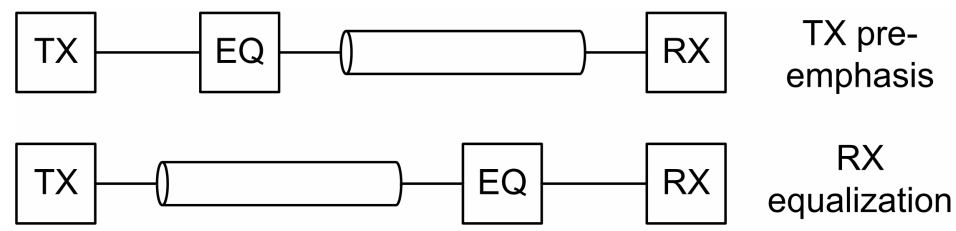

Fig. 11. Transmit (TX) pre-emphasis versus receiver (RX) equalization. 
This filter's transfer function is the inverse of that of the channel, so that the effects of attenuation and dispersion are compensated. Below, we illustrate the concept of an equalizer from a frequency domain point of view. First, we assume that the channel to be equalized has a first order low-pass characteristic, given by

$$
H_{1 s t}(j \omega)=\frac{1}{1+\frac{j \omega}{\omega_{c h}}} .
$$

By placing an equalizing filter in series with the channel, which has the transfer function

$$
H_{e q}(j \omega)=\frac{1+\frac{j \omega}{\omega_{c h}}}{1+\frac{j \omega}{\omega_{p, e q}}},
$$

we can equalize the channel and the equalized channel has the following transfer function:

$$
H_{t o t}=H_{1 s t} \cdot H_{e q}(j \omega)=\frac{1}{1+\frac{j \omega}{\omega_{p, e q}}} .
$$

Thus we can extend the channel bandwidth, provided that $\omega_{p, e q}$ is larger than $\omega_{c h}$. Before equalization, the channel was band-limited to $\omega_{c h} \mathrm{rad} / \mathrm{s}$. After equalization, the $-3 \mathrm{~dB}$ bandwidth is increased to $\omega_{p, e q} \mathrm{rad} / \mathrm{s}$. In the time domain, this has the effect of resolving the ISI. This is discussed in more detail in Chapter 4.

The effect of an equalizer is illustrated in Fig. 12. In Fig. 12(a), the equalizer gain plot is given. In Fig. 12(b), the unequalized and equalized $1^{\text {st }}$ order low pass channel are plotted. In the example, we have chosen the values $\omega_{c h}=150 \mathrm{MHz}$ and $\omega_{p, e q}=2.5 \mathrm{GHz}$. Clearly, the equalized channel is flat over a much wider frequency range, so that the $-3 \mathrm{~dB}$ bandwidth is increased.

For comparison, the measured loss of the $25 \mathrm{~m}$ RG-58CU cable is also plotted. (Note that the frequency axis is now logarithmic instead of linear as before.) The $-3 \mathrm{~dB}$ bandwidth of the cable is only $60 \mathrm{MHz}$. The cable has many poles in the high frequency (HF) range.

When the transfer function of the channel that we want to equalize exhibits deep notches in the signal band (as in Fig. 10), equalization is less useful. An equalizer for such a channel would need to have a very high gain near the notches. It is then unavoidable that the equalizer also amplifies the noise within that frequency range to a high level. A possible solution is to use modulation schemes that avoid putting information at those locations, as we discuss later in this chapter. 


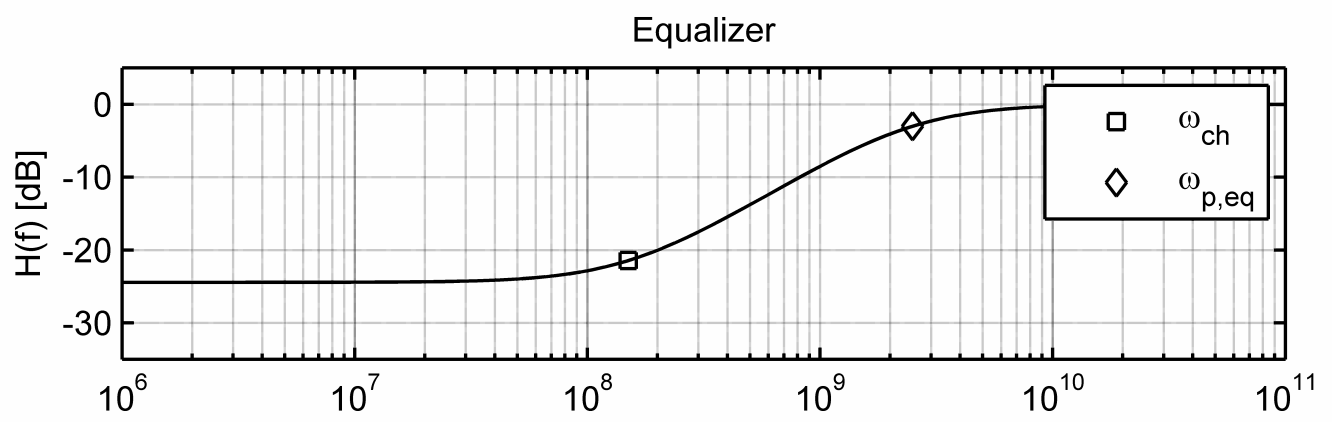

(a)

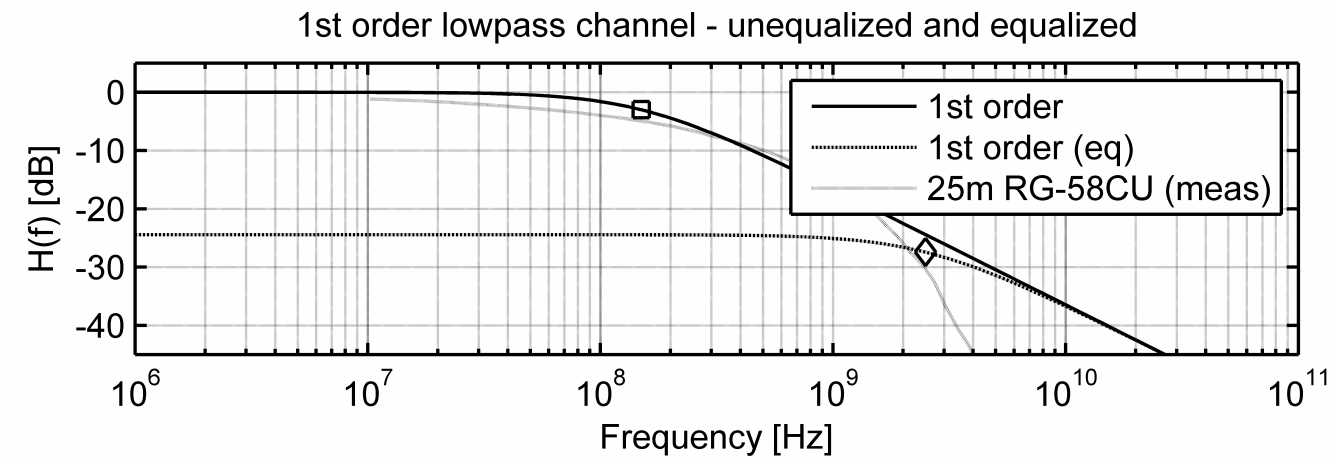

(b)

Fig. 12. Effect of equalizer. (a) Equalizer transfer function. (b) Transfer function of unequalized channel and equalized channel, which is flat over a wider frequency range. Measured loss of a $25 \mathrm{~m} \mathrm{RG-58CU}$ cable is also shown.

\subsubsection{Equalizer gain plot and loss compensation}

From the equalizer gain plot we can read the level of gain (in $\mathrm{dB}$ ) that the equalizer provides at the highest frequency, relative to its DC gain. In the example in Fig. 12(a), the equalizer gain is approximately $24 \mathrm{~dB}$. This gain is often adjustable so that the equalizer can be adapted to the channel.

A figure-of-merit for equalizers that we use throughout this thesis is the loss compensation. It is closely related to the equalizer gain. The loss compensation of the equalizer is equal to the measured channel loss at the Nyquist frequency, under the following conditions. We take a channel of a certain type and length such that the equalizer needs to be set to its maximum gain, while a still acceptable BER is measured at the receive side. This BER is, of course, dependent on the eye opening. A certain eye closure is acceptable as long as the eye closure does not degrade the BER below the acceptable level. (As mentioned above, in general the acceptable BER for high speed links is set very low at $\left.<10^{-12}\right)$.

The loss compensation figure is not dependent on the bit rate that can be achieved by using an equalizer, because of its definition as the channel loss at the Nyquist frequency. We could, for example, have two different equalizers: one for high bit rates and one for low bit rates, with completely different pole and zero locations yet with the same loss compensation. It would only mean that the equalizer for the slower bit rate could transmit information over a longer cable (or over a cable type with more loss per meter). For this reason, it is also important to specify the bit rate that an equalizer can handle. 


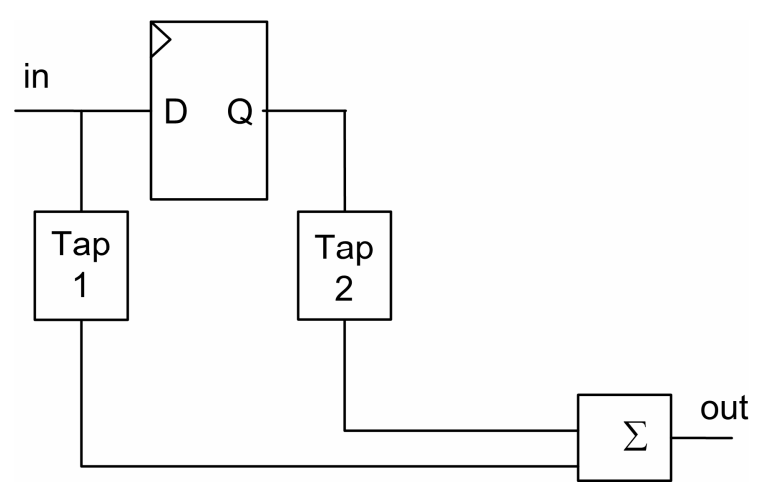

(a)

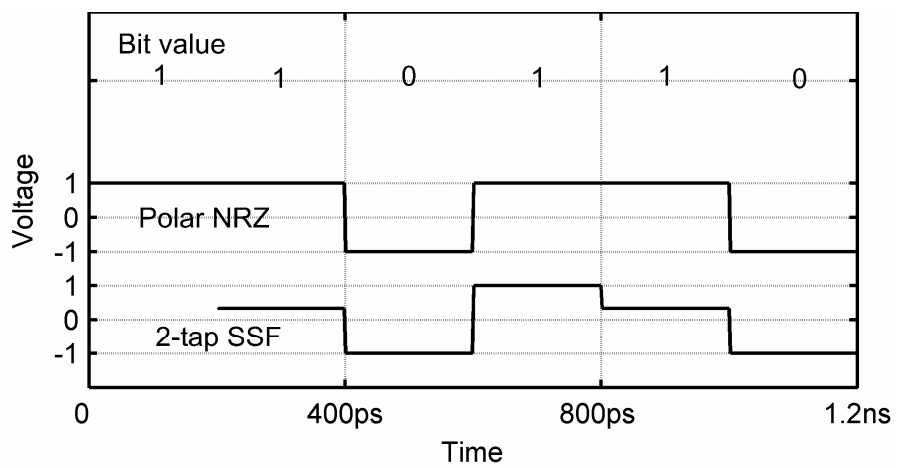

(b)

Fig. 13. A 2-tap symbol spaced FIR pre-emphasis filter and its output. (a) 2-tap SSF filter architecture. (b) Time domain signals for 2PAM (polar NRZ), and for the output of the 2-tap SSF filter.

\subsection{Solutions from the literature}

In this section, we discuss solutions from the literature to the attenuation, dispersion and reflection problems introduced above. Equalization schemes (subsection 1.5.1) and modulation schemes (subsection 1.5.2) are discussed that allow us to approach the theoretical capacity limits of the copper channels.

\subsubsection{Equalizers}

In high-speed serial PAM systems, equalizers are often used to increase the bit rate. In this subsection we discuss the main advantages and disadvantages of proposed equalizer solutions from the literature, to place the work of this thesis in context. There are currently several methods available for achieving a desired equalizer function. First, in 1.5.1.1, the commonly used transmit-side FIR pre-emphasis (PE) is discussed. Next, in 1.5.1.2, receive-side equalizers are discussed.

\subsubsection{FIR PE}

Finite impulse response (FIR) filter-based transmit-side equalization can be implemented in a straightforward way by a FIR-DAC (digital-to-analog converter). Such a FIR-DAC has a digital input and an analog output. In Fig. 13(a), a typical FIR-DAC implementation of a 2-tap symbol-spaced FIR (SSF) pre-emphasis filter is shown. A flip-flop is used to delay the signal with one bit time, and the input and output of this flip-flop are weighed and added to form the 
output. The output is shown in Fig. 13(b), together with the input 2PAM signal. It can be seen that the filter gives a maximum output when there is a transition in the bit stream, either from ' 0 ' to ' 1 ' or vice versa, and when there are no transitions the output is attenuated. This has the effect of attenuating the lower frequencies. Because the transitions are emphasized, this filter is termed a pre-emphasis filter. Actually, de-emphasis is a better word because the equalizer attenuates the lower frequencies to make the transfer function flatter.

Methods described in the literature for equalization of coaxial cables, twisted pair cables and PCBs are commonly based on this FIR-DAC implementation [Lee], [Farjad-Rad], [Dally], [Gai], [Balan], [Stonick], [Zerbe]. Usually the filters are 2-tap symbol-spaced FIR (SSF) filters. However these 2-tap filters typically offer only $10 \mathrm{~dB}$ of loss compensation, e.g. [Lee] at $4 \mathrm{~Gb} / \mathrm{s}$ (2PAM), up to a maximum of approximately $18 \mathrm{~dB}$, e.g. [Kudoh] at $5 \mathrm{~Gb} / \mathrm{s}$ (2PAM).

In [Gai] a 5-tap symbol-spaced equalizer is described that can handle $30 \mathrm{~dB}$ of channel loss at the Nyquist frequency, at $3.125 \mathrm{~Gb} / \mathrm{s}$ (2PAM). Thus higher loss compensation is possible with 5 manually tuned FIR taps. However, the 5-tap implementation imposes strict demands on transistor matching. Deterministic jitter can arise as a result of mismatches. Also, the large number of taps introduces many degrees of freedom that could make it harder for an adaptation algorithm such as LMS to adjust the equalizer to the channel. (For example in [Stonick], $60 \%$ of the $1 \mathrm{~W}$ power budget is spent on the digital blocks that implement the adaptation algorithm.) Furthermore, the number of voltage levels at the output increases by a factor of two with every tap added and linearity demands on the output stage become progressively stricter.

We conclude that 2-tap SSF pre-emphasis, while straightforward to implement, can not provide more than $\sim 20 \mathrm{~dB}$ of loss compensation. Furthermore, we conclude that $30 \mathrm{~dB}$ loss compensation is only achievable using more complex circuits, but which have more parameters that need to be tuned to match the equalizer to the channel.

\subsubsection{RX side Equalizers}

Receiver side equalizers have the advantage that the information to adapt the filter coefficients is readily available. The feedback loop is short and a quick adaptation can be made to changing channel conditions if necessary. We categorize the receiver-side equalizers into two groups: continuous-time equalizers and discrete-time equalizers.

\section{Continuous-time equalizers}

A continuous-time equalizer is - unlike discrete time equalizers - not based on sampling but implements the desired transfer function using an analog time-continuous circuit. We discuss two types of continuous-time equalizers: second-order derivative equalizers and equalizers using RC-degenerated stages.

The second order derivative equalizer works by adding three processed versions of the input [Gai]:

1) the input itself;

2) the derivative of the input;

3) the second order derivative of the input. 


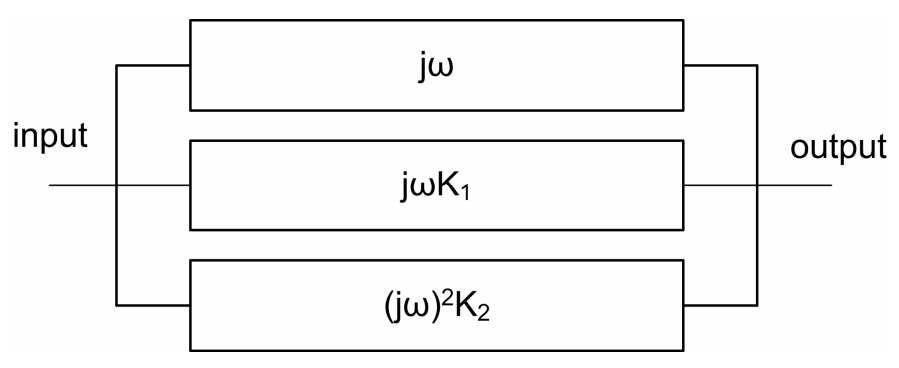

Fig. 14. Second order derivative equalizer.

All these signals are scaled with constants, such that the total output signal can be described as:

$$
H(j \omega)=K_{0}+j \omega K_{1}+(j \omega)^{2} K_{2}
$$

By choosing the optimum constants, a transfer function can be made that closely approximates the inverse of that of the copper interconnect. In each path there are variable gain amplifiers (VGAs) to provide the scaling $K$. Fig. 14 is a conceptual illustration of such a second order derivative equalizer.

Using a second order derivative equalizer, up to $30 \mathrm{~dB}$ of loss compensation is achieved at $3.125 \mathrm{~Gb} / \mathrm{s}$ in [Gai]. However, matching the latencies through the three paths is not straightforward and the circuitry is complex. Furthermore, care has to be taken that the input stage does not become saturated, because in that case the received input signal would show clipping and information would be lost.

An implementation which avoids the use of separate paths is the 'RC-degenerated' implementation [Choi]. Such an architecture uses an RC impedance for source degeneration. Fig. 15 shows a degenerated differential pair. $Z_{2}$ forms the source degeneration. The impedance $Z_{1}$ is usually just a resistor, but $Z_{2}$ incorporates a larger impedance network, which contains one or more poles and sometimes also a zero.

By cascading multiple stages of such RC-degenerated differential pairs, in [Maillard] 30dB of equalizer gain was achieved in $0.18 \mu \mathrm{m}$ CMOS, at a speed of $3 \mathrm{~Gb} / \mathrm{s}$. To obtain this result five stages were needed. Each stage has a $Z_{2}$ impedance that can be switched, to enable adaptation of the equalizer to the channel transfer function. However, this involves a large number of carefully matched poles and zeros (of which a certain number needs to be switched on depending on the channel parameters). Also, the switching order needs to be taken into account. Saturation of the input needs to be prevented, as in the second order derivative equalizer. (This is a general point for receiver equalizers.)

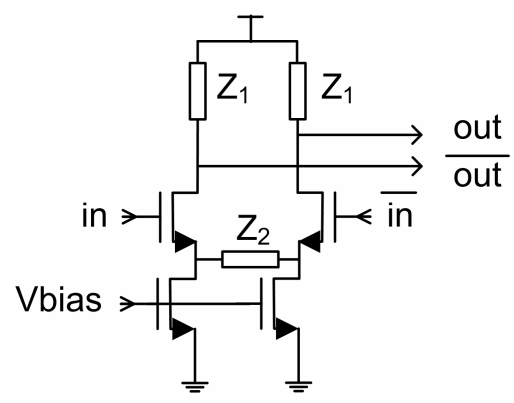

Fig. 15. Single RC-degenerated stage. 
In conclusion, a loss compensation of $30 \mathrm{~dB}$ has been demonstrated at the receiver side, but only with a second order derivative equalizer and with a 5-stage $\mathrm{RC}$ degenerated equalizer.

\section{Discrete-time equalizer: decision feedback equalization}

A discrete-time receive-side equalizer architecture is the decision feedback equalizer (DFE). The concept is illustrated in Fig. 16. In a DFE, decisions of previously detected symbols are subtracted from the input to remove ISI in the current symbol. This has the advantage that the DFE rejects high-frequency noise, because of the 'threshold' decision process [Proakis]. This makes the DFE a good candidate for the noisy environment of 'backplane' PCBs (e.g. those suffering from crosstalk) [Stojanović], [Balan], [Zerbe].

However, the DFE is sensitive to error propagation: when the value of a bit is incorrectly decided upon, it will influence future decisions, because of the feedback [Balan]. Furthermore, at very high bit rates it is challenging to feed back the decisions quickly enough to allow implementation of the first filter tap. Loop unfolding techniques can be used to mitigate this [Zerbe], but this unfortunately also introduces unwanted loading in the critical signal and clock paths, and therefore makes the clock and data recovery (CDR) circuit design more complicated [Stojanović].

A further limitation of the DFE architecture is that only post-cursor ISI can be cancelled, because the bit decisions have to be available to be fed back. For these reasons the achievable loss compensation with DFEs is generally low, and so they are often used in combination with FIR transmit equalizers.

\subsubsection{Modulation: OFDM}

As was shown in Fig. 10, an FR4 'backplane' PCB can exhibit deep spectral nulls in its transfer function, caused by impedance discontinuities. When using PAM for these copper channels, any equalizer would need to have a very high gain at the frequencies with destructive interference, also amplifying the noise to a high level in the process. The information at those frequencies is needed to restore the original signal.

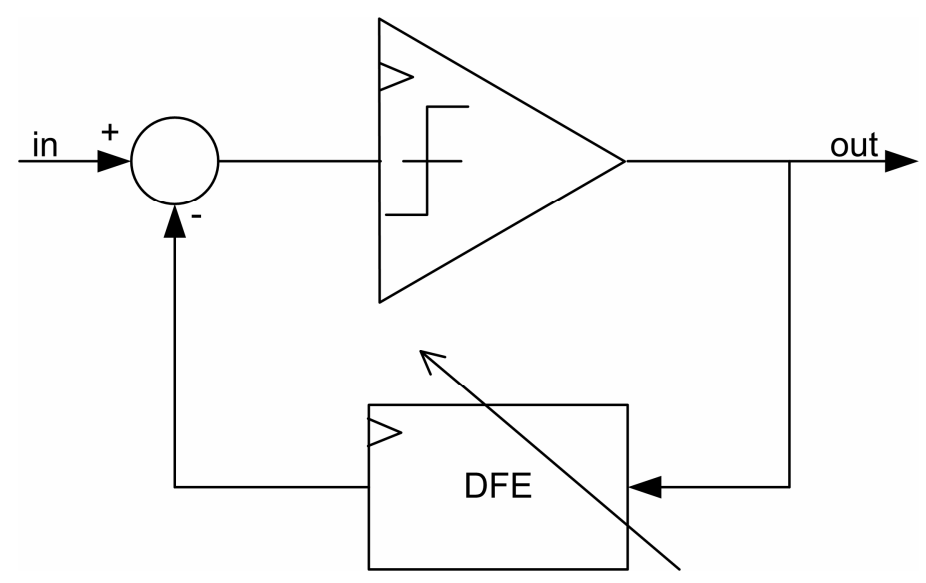

Fig. 16. Decision feedback equalization. 
While we have seen above that DFEs can offer a solution to this 'noise amplification' problem, this is limited because a high enough noise level will eventually cause incorrect decisions in the DFE. In severe cases, it might actually be better to use a modulation method that avoids putting information at those frequencies where notches occur in the channel transfer function. Orthogonal Frequency Division Multiplexing (OFDM) [Weinstein], [Bingham] is, in principle, well suited to this task.

The required data rates in the backplane environment are much higher than those in commonly used and proven digital multi-tone (DMT) implementations of OFDM. For example, data rates achieved over digital subscriber lines (DSL) are around $10 \mathrm{Mb} / \mathrm{s}$. However, to achieve a data rate of $10 \mathrm{~Gb} / \mathrm{s}$ using a DMT implementation would require an extremely high bandwidth, high resolution ADC, and digital signal processing that is currently just not feasible. Therefore the experimental high-speed analog OFDM implementations need to use analog mixers and filters to combine and separate the subcarriers. Solutions of this form are being investigated, e.g. a so-called analog multi-tone (AMT) system [Amirkhany-1]. However, when the local oscillator signals for the mixers experience jitter and duty-cycle deviations, the orthogonality constraints of OFDM are violated. We analyze the impact of such timing nonidealities in this thesis.

\subsection{Research challenges and thesis overview}

In this section the research challenges are summarized (subsection 1.6.1) and an overview of the thesis is provided (subsection 1.6.2).

\subsubsection{Research challenges}

The main goals of this work are:

- to achieve an increase in the bit rate over lossy, dispersive copper channels, over the maximum currently possible,

- to maintain a low Bit Error Rate (BER),

- to implement the above using simple circuits that are feasible to implement in future high-speed CMOS processes.

We have concluded in this introduction that the main problems in communication over copper channels are attenuation, dispersion and reflections, which result in intersymbol interference in the time domain.

Concerning the reflections, we have seen that, for channels with many impedance discontinuities (which lead to deep spectral nulls in the transfer function) an analog OFDM system might offer a solution. However, the high bandwidth necessitates an analog implementation, and we need to investigate what the effect of this implementation is on the OFDM channel orthogonality. Special attention needs to be given to timing non-idealities.

Concerning the attenuation and dispersion arising from conductor and dielectric losses, we have seen that equalization offers a solution. However, equalizer implementations described in the literature that achieve a very high equalizer gain / loss compensation (up to $30 \mathrm{~dB}$ ) can typically only be implemented using complex circuits with multiple degrees of freedom to adjust the equalizer transfer function to the channel. We would like to develop a simpler equalizer that decreases this number of parameters while still providing a high loss compensation. 
Finally, for effective numerical computer simulations of the new techniques, we need an accurate time domain model of the channels.

\subsubsection{Thesis overview}

In Chapter 2, we review existing channel models, with a focus on transient (time-domain) simulations using copper cables and PCBs. We look into recent publications to find a model for the dielectric loss which improves on those described in current textbooks (which typically give the frequency dependent loss tangent only at certain points). Special attention is given to causality, so as to provide an accurate impulse response for use in high speed transient simulations. The selected model is fitted with and compared to measurements.

In Chapter 3, we analyze whether OFDM is a useful modulation method for data transmission over channels that suffer from severe reflections. An analog high-bandwidth OFDM system is described and its performance is analyzed. We focus on the sensitivity to timing non-idealities such as jitter and duty-cycle deviations. It is expected that these timing non-idealities may cause serious inter-carrier interference (ICI) in OFDM systems, which limits the achievable bit rate.

In Chapter 4, we look at alternative equalization techniques to increase the bit rate over copper channels. It is shown that a simple pulse-width modulation (PWM) pre-emphasis technique works well on copper channels, achieving up to $30 \mathrm{~dB}$ loss compensation. We analyze PWM pre-emphasis in the time and frequency domains and compare the loss compensation to that achieved using a conventional FIR pre-emphasis filter. The design of three proof-of-concept transmitter chips is described. Measurement results are given for both coaxial and differential cables, and for a printed circuit board.

In Chapter 5, the PWM pre-emphasis technique is extended to a multitap version. As with a multitap FIR filter this enables us to make more complex transfer functions. The power spectral density functions of the multitap PWM filters are calculated using their autocorrelation functions, and time domain simulation results are given.

In Chapter 6, the thesis is summarized, the original contributions are listed, and suggestions are given for future research topics. 


\section{Chapter 2}

\section{Modeling copper cables and PCBs}

\subsection{Introduction}

A proper channel model is a good basis for a correct understanding of any transmission system. The goal of this chapter is to identify an accurate time-domain model with a focus on high-speed time-domain (transient) simulations. Both conductor and dielectric loss need to be modeled. We compare the model with measurements on cables and printed circuit boards (PCBs) in the time and frequency domains to be able to assess its accuracy.

Well-known textbooks [Gardiol], [Grivet], [Chipman], [Ramo] typically describe accurate models for the skin-effect magnitude and phase shift, and some also describe an analytical skin-effect step response. However, the modeling of dielectric loss at high frequencies is still a developing field and has received much attention recently. For high-speed serial links over printed circuit boards, accurate modeling is especially important. Modeling the complex dielectric permittivity and the loss tangent as frequency-independent values leads to noncausal results, which is especially problematic when accurate time-domain simulations are the goal [Hall], [Djordjević]. Therefore we focus on identifying a causal time-domain model. As a basis, we use the Kramers-Kronig relations [Ramo], which express a mathematical relationship between real and imaginary parts of the response functions. In this chapter, we use a recently published model for dielectric loss in printed circuit boards [Djordjević] to model the dielectric part of the cable loss. It is shown that the combination of the skin-effect model and this dielectric model gives a causal time-domain impulse response, using a minimum of model parameters.

Models only have meaning if they can accurately predict measurement results, so we need to thoroughly check the model predictions. We describe two types of measurements in this chapter: network analyzer measurements and time-domain Transmissometry (TDT) measurements. Each type of measurement has its particular use. A magnitude transfer plot gives direct insight into the channel loss at a certain frequency, for example at the Nyquist frequency. The time domain impulse response, on the other hand, gives a direct intuitive insight into intersymbol interference (ISI).

Concerning the frequency domain, measurement of the magnitude is more straightforward than of the phase. Due to the long propagation delay of the long cables that we use, and the high signal frequencies, the phase rotates very rapidly. Using this data to obtain the cable impulse response using an inverse Fourier transform can be hard, since the data needs to be corrected for noise and measurement artifacts. We can, of course, construct the 'minimumphase' as described in [Bode] but without measuring we cannot be sure whether the cable is actually a minimum-phase network (after subtraction of the propagation delay). Therefore, we wish to avoid the need for accurate phase measurements. Our aim is to find a model which can be matched with only the measured magnitude, and then, after fitting the model, to obtain the phase from the model. This magnitude and phase information is then input to the inverse Fourier transformation. 
Concerning the time domain, wideband $(10 \mathrm{GHz}$ and higher) time-domain equipment has recently become available that enables direct measurement of the step response. Through differentiation, the impulse response can be obtained. The differentiation adds noise to the measurements, and the generated step has a limited rise time. This limits the accuracy of the time domain measurements so it is important to have frequency domain measurements as well. We fit the model with the measured frequency domain magnitude data and check it in the time domain against the measured impulse response. Measurements in both domains give more certainty in assessing the model's accuracy.

In this chapter, we model and measure coaxial cables, twisted pair cables, and printed circuit boards. It is shown that both the skin effect equations and the dielectric equations obey the Kramers-Kronig relations, guaranteeing causality.

In section 2.2 we first define the transfer function and the complex propagation constant in the RLGC form, and the constraints imposed on it by causality. Next, in section 2.3, all the RLGC parameters, and then the complete frequency domain transfer function (magnitude and phase) are calculated by analyzing both the skin effect and the dielectric loss. The subject of section 2.4 is the time domain impulse responses. Next, in section 2.5, measurement results are given for cables and printed circuit boards, and compared to the model.

\subsection{Propagation constant and Kramers-Kronig relations}

\subsubsection{Propagation constant}

The frequency domain transfer function $H(j \omega)$ of a perfectly matched lossy copper transmission line is given by:

$$
H(j \omega)=e^{-\gamma l},
$$

where $l$ is the length of the cable, and $H(j \omega)$ is defined as the ratio between the output and input voltages of the cable ( $V_{\text {out }}$ and $V_{\text {in }}$ respectively):

$$
H(j \omega)=\frac{V_{\text {out }}(j \omega)}{V_{\text {in }}(j \omega)} .
$$

The complex propagation constant $\gamma$ is defined as [Gardiol], [Grivet], [Chipman], [Ramo]:

$$
\gamma=\sqrt{(R+j \omega L)(G+j \omega C)},
$$

where $\mathrm{R}$ is the distributed series resistance $(\Omega / \mathrm{m}), \mathrm{L}$ is the distributed inductance $(\mathrm{H} / \mathrm{m}), \mathrm{G}$ the distributed parallel conductance $(\mathrm{S} / \mathrm{m})$, and $\mathrm{C}$ the distributed capacitance $(\mathrm{F} / \mathrm{m})$, as shown in Fig. 1.

The actual values of the RLGC parameters are determined in the next sections, by analyzing the skin effect and the dielectric loss. 


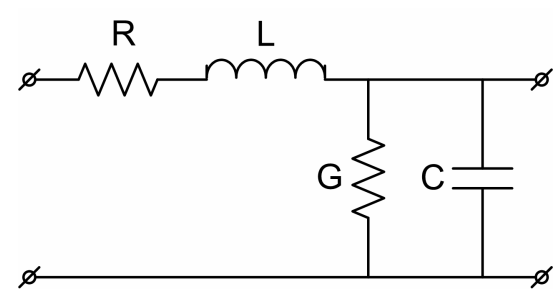

Fig. 1. RLGC representation of an infinitesimally small section of the transmission line.

\subsubsection{Kramers-Kronig relations}

The transfer function $H(\omega)$ is subject to a strict set of rules, because it is the Fourier transform of the transmission line's impulse response. This transmission line is a physical system, which imposes the two constraints described below.

(1) A physical system can only have a causal impulse response (no reaction before an action), so the following needs to be true:

$$
h(t)=0 \quad t<0
$$

This causality requirement for $h(t)$ enforces a strict relation between the real and imaginary parts of its Fourier transform. This Fourier transform is a complex analytic function in the upper half plane.

(2) The impulse response of a physical system is a real function. As a result of this, its Fourier transform $H(\omega)$ has a special property. The values at positive frequencies are the complex conjugate of the values at negative frequencies:

$$
H(-\omega)=H^{*}(\omega) .
$$

When condition (1) is valid, the Kramers-Kronig relations apply to the real and imaginary parts of the Fourier transform [Ramo]. These mathematical relations connect the real and imaginary parts of a complex analytic function in the upper half plane for the response function [Arabi], [Djordjević]. They allow us to calculate the imaginary part of the response, knowing only the real part, and vice versa. They are a special case of the Hilbert transform [Djordjević]. The symmetry implied by condition (2) simplifies the Kramers-Kronig relations such that their integration interval runs from zero to plus infinity, instead of from minus infinity to plus infinity. For the complex function:

$$
X(\omega)=X_{r}(\omega)+i \cdot X_{i}(\omega)
$$

the simplified Kramers-Kronig relations are [Ramo]:

$$
X_{r}(\omega)=\frac{2}{\pi} P V \int_{0}^{\infty} \frac{\omega^{\prime} X_{i}\left(\omega^{\prime}\right)}{\omega^{\prime 2}-\omega^{2}} d \omega^{\prime}
$$

and 


$$
X_{i}(\omega)=-\frac{2}{\pi} P V \int_{0}^{\infty} \frac{\omega X_{r}\left(\omega^{\prime}\right)}{\omega^{\prime 2}-\omega^{2}} d \omega^{\prime}
$$

where PV denotes the Cauchy principal value, necessary for calculation because the functions have a discontinuity at $\omega^{\prime}=\omega$.

We use the Kramers-Kronig relations throughout the rest of this chapter, to make sure that we obtain a causal impulse response.

\subsection{Frequency domain equations}

In this section, we calculate the values for the RLGC parameters. First, in subsection 2.3.1, ' $\mathrm{R}$ ' and ' $\mathrm{L}$ ' is calculated by analyzing the skin effect, and next, in subsection $2.3 .2,{ }^{\prime} \mathrm{C}$ ' and ' $\mathrm{G}$ ' is calculated by analyzing the dielectric loss.

\subsubsection{Conductor losses: the skin effect}

The skin effect is the phenomenon that the penetration depth of electromagnetic (EM) waves into a non-ideal conductor is dependent on the frequency, leading to a frequency dependent series resistance and inductance. High-frequency current flows only in the skin of the conductor; hence the term "skin effect". It has effects on ' $R$ ' and on ' $L$ ': the decreasing EM wave penetration depth reduces the effective usable conductor area, while it also decreases the internal inductance (internal to the wire) and the magnetic field caused by this current. The skin effect was accurately described as long ago as 1934 [Schelkunoff]. For completeness, and for later use in our measurements, we give the formulas below.

In 2.3.1.1 we calculate the distributed resistance $\mathrm{R}$ and in 2.3.1.2 the distributed inductance $\mathrm{L}$. Next, in 2.3.1.3, we show that the Kramers-Kronig relations hold for the complex impedance formed by $\mathrm{R}$ and $\mathrm{L}$.

\subsubsection{R: distributed resistance}

In our analysis we first look at the effective conductor resistance. This resistance increases continuously with frequency. The current density decreases gradually from the skin to the conductor center. The effective wave penetration depth $\partial_{s}$, defined as the penetration distance at which the current density is attenuated by 1 neper $(8.69 \mathrm{~dB})$, is related to angular frequency, electric conductivity $\sigma$ and magnetic permeability $\mu$ as follows [Gardiol], [Grivet], [Chipman], [Ramo]:

$$
\partial_{s}=\sqrt{\frac{2}{\omega \mu \sigma}} .
$$


Combining penetration depth with wire radius, the skin effect cutoff angular frequency $\omega_{s a}$ (where the skin depth is equal to the radius) can be calculated for a circular conductor [Gardiol], [Grivet], [Chipman], [Ramo]:

$$
\omega_{s a, x}=\frac{2}{x^{2} \mu \sigma},
$$

where $x$ is the radius of the conductor. The cutoff frequency is independent of cable length.

Well below this cutoff frequency, the EM waves use the whole conductor area and the distributed conductor resistance (without return path) is equal to its DC level [Gardiol], [Grivet], [Chipman], [Ramo]:

$$
R_{D C, \sin g l e}=\frac{1}{\sigma \pi x^{2}}
$$

For example, for the RG-58U coaxial cable, the cutoff frequency is as low as $22 \mathrm{kHz}$. Above the cutoff frequency, the distributed $\mathrm{AC}$ wire resistance $R_{A C}$ can be calculated as [Gardiol], [Grivet], [Chipman], [Ramo]:

$$
R_{A C}=\lambda \sqrt{\omega}
$$

For a coaxial cable, $\lambda$ is equal to [vdPlaats]:

$$
\lambda_{c}=\frac{1}{2 \pi}\left(\frac{1}{a}+\frac{1}{b}\right) \sqrt{\frac{\mu}{2 \sigma}},
$$

where $a$ is the radius of the center conductor, and $b$ the distance from the center to the shield (see Fig. 2(a)). For the differential pair [vdPlaats]:

$$
\lambda_{d}=\frac{2 D}{\pi d \sqrt{D^{2}-d^{2}}} \sqrt{\frac{\mu}{2 \sigma}},
$$

where $d$ is the diameter of the either one of the two conductors and $D$ is the distance between the centers of the two conductors (see Fig. 2(b)).

For a PCB microstrip, the $\lambda$ parameter is given by [Svensson]:

$$
\lambda_{p}=\frac{1}{w} \sqrt{\frac{\mu}{2 \sigma}}
$$

where $w$ is the track width. 


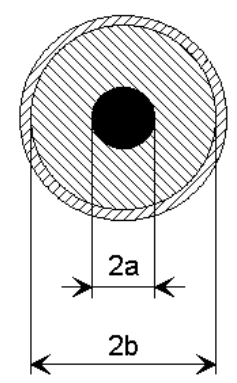

(a)

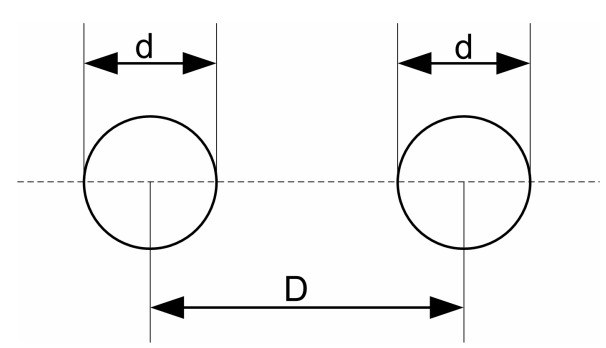

(b)

Fig. 2. Cable cross-sections. (a) Coaxial cable. (b) Differential pair.

\subsubsection{L: distributed inductance}

The total distributed conductor inductance $L$ consists of an internal and external component [Gardiol], [Grivet], [Chipman], [Ramo]:

$$
L=L_{e}+L_{i}
$$

The frequency-independent external inductance $L_{e}$ characterizes the relation between external flux and total current in the conductor, and can be estimated for the coaxial cable as [Gardiol], [Grivet], [Chipman], [Ramo]:

$$
L_{e, c}=\frac{\mu}{2 \pi} \ln (b / a)
$$

and for the differential pair [Gardiol], [Grivet], [Chipman], [Ramo]:

$$
L_{e, d}=\frac{\mu}{\pi} \cosh ^{-1}\left(\frac{D}{d}\right) .
$$

For estimating the distributed external inductance of the PCB microstrip, we can use the well known design rule [Johnson]:

$$
L_{e, s}=2 \cdot 10^{-7}\left(\frac{5.98 d}{0.8 w+h}\right),
$$

where $d$ is the trace height above the groundplane (dielectric distance) and $h$ is the trace thickness. 
The internal inductance $L_{i}$ of the conductor is the relation between internal flux and current inside the conductor, and it is affected by the skin effect. This $L_{i}$ is calculated as [Gardiol], [Grivet], [Chipman], [Ramo]:

$$
L_{i}=\frac{\lambda}{\sqrt{\omega}} \text {. }
$$

From Eq. 20, it can be seen that $L_{i}$ decreases continuously with frequency.

\subsubsection{Kramers-Kronig relations between $\mathrm{R}$ and $\mathrm{Li}$}

The Kramers-Kronig relations can be applied to the skin-effect formulas for $R$ and $L_{i}$. For this purpose, we define the internal (skin) impedance $Z_{i}$ as follows:

$$
Z_{i}=R+j \omega L_{i}
$$

where $R$ the resistance and $L_{i}$ the internal inductance. Described in a different way, it is the ratio of the longitudinal potential difference over a unit length of the conductor to the longitudinal current in the conductor [Chipman]. To show that the internal impedance satisfies the Kramers-Kronig relations, we equate it with Eq. 6:

$$
R+j \omega L_{i}=X_{r}(\omega)+j X_{i}(\omega)
$$

Substituting $X_{r}(\omega)=\lambda \sqrt{ }(\omega)$ in Eq. 8, we obtain [vEtten]:

$$
X_{i}(\omega)=-\frac{2}{\pi} P V \int_{0}^{\infty} \frac{\omega \lambda \sqrt{\omega^{\prime}}}{\omega^{\prime 2}-\omega^{2}} d \omega^{\prime}=\lambda \sqrt{\omega},
$$

which shows that the causality condition is satisfied, because:

$$
j \omega L_{i}=j \omega \frac{\lambda}{\sqrt{\omega}}=\lambda \sqrt{\omega}=j X_{i}(\omega)
$$

(Compare Eq. 20.)

Alternatively, the relation can be considered from the viewpoint of the conservation of energy [Ramo]. The internal (skin) impedance has to obey the law of the conservation of energy. The energy that is dissipated by the resistance cannot be stored in the magnetic field [Hall]. Thus when the resistance increases with increasing frequency, the internal inductance needs to decrease.

With a real conductance $\sigma$, the resistance and internal reactance of a plane conductor are equal at any frequency [Ramo]. Therefore the internal impedance always has a phase angle of 45 degrees.

\subsubsection{Dielectric Loss}

Continuing the calculation of the RLGC parameters, we now calculate the ' $\mathrm{C}$ ' and ' $\mathrm{G}$ ' parameters by analyzing the second loss effect: the dielectric loss. 
The dielectric loss is caused by the conversion of electrical energy to other domains by the dielectric between the two conductors in the cable, mainly as a consequence of dielectric polarization and relaxation.

A crossing frequency can be found beyond which the dielectric loss starts to predominate over the skin effect. The skin effect dominates at low frequencies in any cable, and dielectric loss dominates at high frequencies. Therefore, for cables errors in modeling the dielectric loss are usually not very visible. For high speed serial links over printed circuit board traces, the situation is different. The dielectric in FR4, made from glass fiber and epoxy, has much higher loss than the polyethylene or specially designed low-loss (foamed or air) dielectric in cables. Therefore, in FR4, the dielectric loss predominates over the skin loss already at low frequencies. The recent work on high-speed broadband cable and backplane datacommunication systems has revitalized interest in channel models that remain accurate in the $\mathrm{GHz}$ range. Indeed, it is a very topical subject: recently many publications have been devoted to the challenge of modeling dielectrics [Djordjević], [Hall]. Jonscher wrote in 1999 that 'dielectric relaxation in solids represents one of the most intensely researched topics in physics' [Jonscher].

In 2.3.2.1, the distributed capacitance $\mathrm{C}$ is calculated. In 2.3.2.2, the distributed shunt conductance $\mathrm{G}$ is calculated, and the modeling of the loss tangent is discussed.

\subsubsection{C: the distributed capacitance}

For calculating the distributed capacitance $\mathrm{C}$, we first introduce the frequency-dependent complex dielectric permittivity $\varepsilon$, which is dependent on the dielectric material:

$$
\varepsilon(\omega)=\varepsilon^{\prime}-j \varepsilon^{\prime \prime}
$$

The distributed parallel capacitance in a coaxial cable is given by [Gardiol], [Grivet], [Chipman], [Ramo]:

$$
C_{c}(\omega)=\frac{2 \pi \varepsilon^{\prime}}{\ln (b / a)}
$$

and, for a differential pair, by [Gardiol], [Grivet], [Chipman], [Ramo]

$$
C_{d}(\omega)=\frac{\pi \varepsilon^{\prime}}{\cosh ^{-1}\left(\frac{D}{d}\right)}
$$

For the estimating the distributed capacitance of the PCB microstrip, we can use the well known design rule [Johnson]:

$$
C_{s}(\omega)=\frac{2.64 \cdot 10^{-11}\left(\frac{\varepsilon^{\prime}}{\varepsilon_{0}}+1.41\right)}{\ln \left(\frac{5.98 d}{0.8 w+h}\right)}
$$




\subsubsection{G: distributed shunt conductance}

The last remaining RLGC parameter is $\mathrm{G}$, the distributed shunt conductance. It is calculated using the frequency-dependent loss tangent. The definition of the loss tangent is:

$$
\delta(\omega)=\frac{\varepsilon^{\prime \prime}}{\varepsilon^{\prime}}
$$

The frequency-dependent shunt conductance per meter $\mathrm{G}$ is given by [Gardiol], [Grivet], [Chipman], [Ramo]:

$$
G(\omega)=\delta \omega C
$$

In some older publications [Brianti], [vdPlaats], the loss tangent is incorrectly assumed to be a constant and as a result, the dielectric part of the transfer function is modeled as linearly dependent on frequency and considered to be non-dispersive. An inverse Fourier transform then (incorrectly) yields a symmetrical impulse response. A dielectric attenuation linearly dependent on $\omega$ is physically impossible. The Paley-Wiener criterion and Kramers-Kronig relations dictate that no causal network can provide an attenuation which (asymptotically) increases as a function of $\omega$ with a factor larger than or equal to one times $\omega$ [Guillemin], [Nahman].

To guarantee causality in the time domain, the real and imaginary parts of the complex dielectric permittivity function again need to form a Kramers-Kronig pair [Ramo]. This can also be understood from another point of view. The conservation of energy law also dictates a necessary relation between $\varepsilon$ ' and $\varepsilon$ ". The energy that is dissipated in the dielectric cannot be stored on its capacitance. When the real part of the dielectric permittivity decreases, less energy can be stored capacitively and the dielectric losses increase, indicated by an increase in the imaginary part of the dielectric constant [Hall].

To obtain a dielectric response that does comply with Kramers-Kronig, the response can be modeled as a sum of multiple single Debye responses, each with a different relaxation time [Svensson], or a continuous sum of an infinite number of Debye responses [Djordjević]. Although [Jonscher] notes that this distributed relaxation times approach "fails to address the evident existence of a universal fractional power-law behavior which represents very well the high-frequency dipolar behavior", at present it does seem to be the most practical approach. In their papers, [Svensson] and [Djordjević] show their model to correspond well with measurements.

The complex, frequency-dependent dielectric permittivity of FR4 is approximated as follows [Djordjević], as published in 2001:

$$
\varepsilon(\omega)=\varepsilon^{\prime}-j \varepsilon^{\prime \prime}=\varepsilon_{\infty}^{\prime}+\frac{\Delta \varepsilon^{\prime}}{m_{2}-m_{1}} \log _{10}\left(\frac{\omega_{2}+j \omega}{\omega_{1}+j \omega}\right),
$$

where $\Delta \varepsilon^{\prime}$ is the total variation of $\varepsilon$ between the lower $\left(\omega_{1}=10^{\mathrm{m} 1}\right)$ and upper model frequency $\left(\omega_{2}=10^{\mathrm{m} 2}\right)$, and $\varepsilon_{\infty}^{\prime}$ is the dielectric constant at very high frequencies. This equation for $\varepsilon$ obeys the Kramers-Kronig relations. 
Although the above function is meant for modeling the complex dielectric permittivity in FR4, it was also found to be useful and accurate to model the dielectric in cables. We use Eq. 31 to calculate the frequency-dependent values for $C$ and $G$. The optimum values for the parameters in Eq. 31 are determined experimentally, to fit with the measurements.

\subsubsection{Isolated magnitude transfer function for skin loss and dielectric loss}

It is important to be able to see in the frequency domain magnitude plot the separate contributions of skin-effect and dielectric-loss. We could, for example, determine which loss mechanism is dominant and at which 'crossing frequency' the dielectric loss starts to dominate over the skin-effect loss.

Having found all the RLCG parameters, we can collect them together and substitute them into Eq. 3 for the propagation constant, which leads to:

$$
\gamma=\sqrt{\left(\lambda \sqrt{\omega}+j \omega\left(L_{e}+\frac{\lambda}{\sqrt{\omega}}\right)\right)(\delta \omega C+j \omega C)},
$$

where $C$ is frequency-dependent through $\varepsilon$ (using Eq. 31), and $\delta$ is also frequency-dependent. (See Eq. 29.) The above formula can be rewritten as

$$
\gamma=j \omega \sqrt{L_{e} C\left(1+\frac{(1-j) \lambda}{L_{e} \sqrt{\omega}}\right)(1-j \delta)} .
$$

The loss contributions in the propagation constant can be separated by using a binomial series approximation. Using the binomial series for power 0.5 , valid for small $x$ :

$$
\sqrt{1+x}=1+\frac{x}{2}-\frac{1}{8} x^{2}+\frac{1}{16} x^{3}+\ldots
$$

we obtain [vdPlaats]

$$
\gamma=j \omega \sqrt{L_{e} C}\left(1+\frac{(1-j) \lambda}{2 L_{e} \sqrt{\omega}}-\frac{(1-j)^{2} \lambda^{2}}{8 L_{e}^{2} \omega}+\ldots\right)\left(1-j \frac{\delta}{2}+\frac{\delta^{2}}{8}+\ldots\right)
$$

The series can be truncated after the $x / 2$ term, because the further terms are negligible, resulting in [vdPlaats]:

$$
\gamma=\underbrace{j \omega \sqrt{L_{e} C}+j \frac{\lambda}{2} \sqrt{\frac{C}{L_{e}}} \sqrt{\omega}}_{j \beta}+\underbrace{\frac{\lambda}{2} \sqrt{\frac{C}{L_{e}} \sqrt{\omega}+\frac{\delta \omega}{2} \sqrt{L_{e} C}}}_{\alpha},
$$

where the accolades indicate the loss terms $\alpha$ and the phase terms $\beta$.

(As an aside - because $C$ is a (weak) function of $\omega$, because it is calculated using Eq. 31, as stated above, we cannot simply state that the propagation delay is given by the first phase term in the above equation. In fact, that term also captures the dispersion from the dielectric loss. This is not a problem here because we are only interested in the magnitude.) 
We take the loss terms (the third and forth terms) because we are interested in the magnitude transfer function. The frequency domain magnitude transfer function is now written as:

$$
|H(\omega)|=e^{\left(\frac{\lambda}{2} \sqrt{\frac{C}{L_{e}}} \sqrt{\omega}+\frac{\delta \omega}{2} \sqrt{L_{e} C}\right) l} .
$$

In this equation, we can see the separate contributions for the skin-effect loss and the dielectric loss. To see only the dielectric loss, we set the conductivity $\sigma$ to infinity, so that $\lambda=0$. The dielectric loss contribution is then:

$$
\left|H_{\text {diel }}(\omega)\right|=e^{\left(\frac{\delta \omega}{2} \sqrt{L_{e} C}\right) l} .
$$

To see only the skin-effect loss, we set the loss tangent $\delta$ to zero, resulting in:

$$
\left|H_{\text {skin }}(\omega)\right|=e^{\left(\frac{\lambda}{2} \sqrt{\frac{C}{L_{e}}} \sqrt{\omega}\right) l} .
$$

\subsection{Time domain equations}

The impulse response can be obtained from the transfer function from Eq. 1 above by a (numerical) inverse Fourier transform. We use this in the measurement section. However, for quick time-domain simulations, it can also be useful to have an analytical formula for the impulse response. In this section, we deal with the impulse responses for the skin effect (subsection 2.4.1) and for the dielectric loss (subsection 2.4.2).

\subsubsection{Skin-effect impulse response}

Well known from the literature is the skin-effect step response $a(t)$ [Gardiol], [Grivet], [Chipman], [Ramo]:

$$
a(t)=\operatorname{erfc}\left(\sqrt{\tau_{1} / t} / 2\right)
$$

This function is illustrated in Fig. 3(a). By differentiating this equation we obtain the (causal) skin-effect impulse response $h_{l}(t)$ [vdPlaats]:

$$
h_{1}(t)=\frac{\sqrt{\tau_{1}}}{2 t \sqrt{\pi t}} \cdot e^{-\frac{\tau_{1}}{4 t}}
$$

where the skin-effect time constant $\tau_{1}$ is equal to:

$$
\tau_{1}=\frac{l^{2} \lambda^{2}}{2 Z_{c}^{2}},
$$

where $Z_{c}=\sqrt{L_{e} / C}$ approximates, at high frequencies, the characteristic impedance. 
Fig. 3(b) shows $h_{l}(t)$. The x-axis shows time divided by $\tau_{l}$. The y-axis shows $h_{l}(t) \cdot \tau_{l}$. The axis is chosen in this way to clearly show the maxima and time span of the function. It can be seen that $h_{1}(t)$ is asymmetrical over time with a very long tail.

In fact, 'time constant' $\tau_{1}$ is not constant but is dependent on frequency through the frequencydependent electrical capacity $\mathrm{C}$. We test the accuracy of this simplification later in this chapter, but for now we state that for channels that are dominated by skin-effect loss, such as high-quality cables, $h_{l}(t)$ as given above is an accurate approximation of the impulse response.

In Chapter 4 we use this analytical skin-effect impulse response in time-domain simulations to analyze the PWM equalizer.

As an interesting aside, in Appendix A it is shown that a characteristically terminated on-chip $\mathrm{RC}$ line has exactly the same response as a skin-effect-only channel. Both channels can be described by means of a diffusion equation.

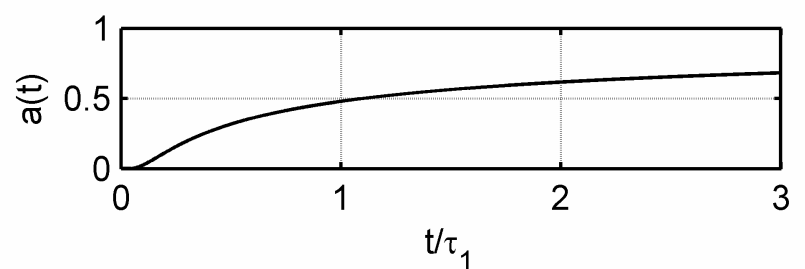

(a)

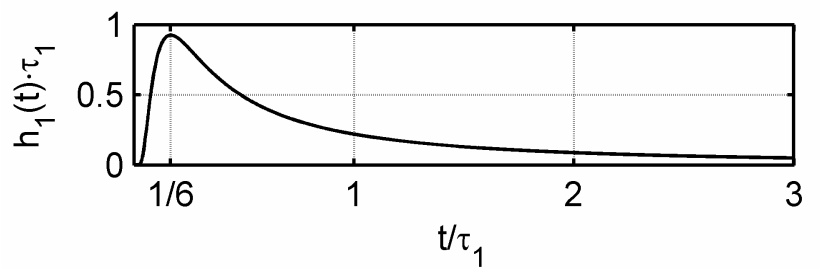

(b)

Fig. 3. Skin effect step response and impulse response. (a) Step response. (b) Impulse response.

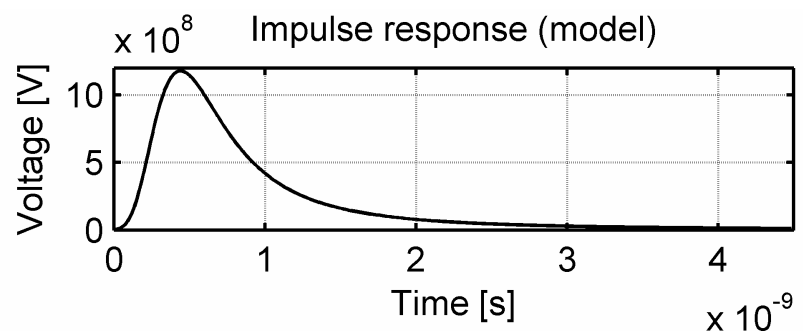

Fig. 4. Example of the theoretical, isolated impulse for dielectric loss. 


\subsubsection{Impulse response for dielectric loss}

Unlike the skin-effect impulse response, the analytic impulse response of a channel dominated by dielectric loss could not be found in the literature. For an intuitive insight into the behavior of these channels, it would be convenient to know which shape this impulse response has. To obtain the impulse response, we first set the skin loss to zero by setting the conductivity to infinity, which results in $R=0$ and $L_{i}=0$. The propagation constant then becomes:

$$
\gamma_{\text {diel }}=\sqrt{\left(j \omega L_{e}\right)(G+j \omega C)},
$$

and the accompanying dielectric-only transfer function is:

$$
H_{\text {diel }}(j \omega)=e^{-\gamma_{\text {diel }} l} .
$$

Taking the inverse Fourier transform of this equation yields the isolated dielectric impulse response. Like the skin-effect impulse response, it is also asymmetrical over time, with a long tail on the right side. The rise time is a bit longer and the function is less steep on the left side. See Fig. 4 for an illustration of an example. (In that figure, the propagation delay is subtracted from the time.)

\subsection{Match between model and measurements}

In this section, we compare the model with measurements of copper channels. Both time domain and frequency domain measurements are used. Measurements are made on four cables and a printed circuit board trace. First, in subsection 2.5.1, the measured channels are described. Next, in subsection 2.5.2, the measured transient responses to a random sequence of bits are given. Finally, in subsection 2.5.3, the measurements of the transfer functions $S_{21}$ and the step responses are discussed and we check how well the model results match the measurements.

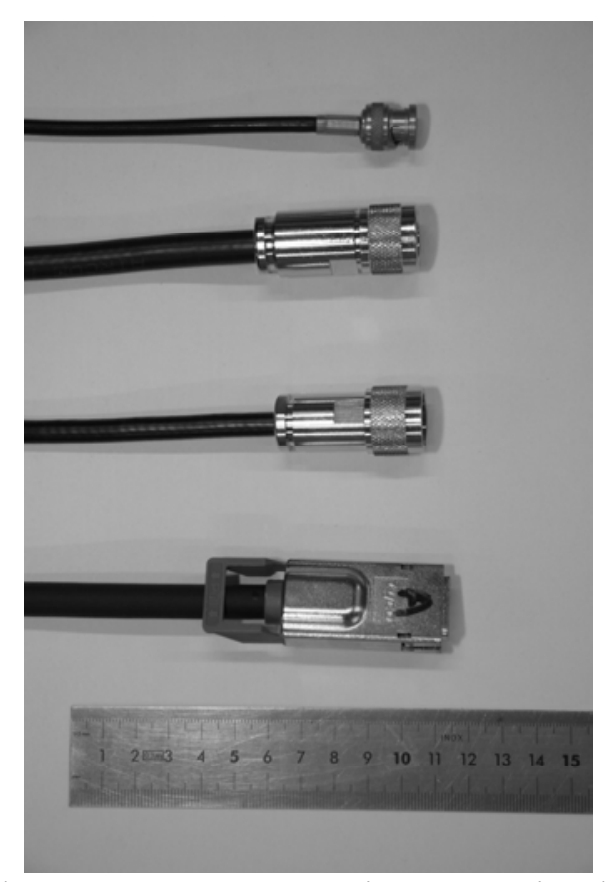

Fig. 5. From top to bottom: RG-58CU, Aircom+, Aircell7, 10GBASE-CX4. 


\subsubsection{Copper channel types}

We measure four copper cables and one printed circuit board. We use two types of copper cables in our measurements: coaxial and differential cables. A photo of the cables and connectors is shown in Fig. 5, and the PCB trace is shown in Fig. 6. The five channels are:

(a) $25 \mathrm{~m}$ RG-58CU (coaxial cable)

(b) $130 \mathrm{~m}$ Aircom + (coaxial cable)

(c) $80 \mathrm{~m}$ Aircell7 (coaxial cable)

(d) $15 \mathrm{~m}$ 10GBASE-CX4 (24AWG shielded differential cable)

(e) $270 \mathrm{~cm} 50 \Omega$ FR4 PCB microstrip.

Table 1 shows the channel parameters and the parameters of the model fit, described later in the measurement section of this chapter. Cable (a) is a low-cost, low-end, standard coaxial cable with polyethylene dielectric. Cable (b) is a more expensive coaxial cable with low-loss air dielectric, designed for frequencies up to $10 \mathrm{GHz}$. However, it is rather rigid and has a large diameter. Cable (c) has a foam dielectric and an inner conductor of woven copper. Therefore it is much more flexible and thinner than (b), while still offering a low dielectric loss relative to cable (a). Finally cable (d) contains 8 shielded differential pairs and is designed for a bit rate of $3.125 \mathrm{~Gb} / \mathrm{s}$ per pair using 2-tap SSF at the transmitter. A small adapter PCB makes one of the pairs available at an SMA connector. All coaxial cables and the PCB trace have a characteristic impedance of $50 \Omega$ and (d) has a differential characteristic impedance of $100 \Omega$. Cable (d) was not available at lengths of more than $15 \mathrm{~m}$. Channel (e) is a printed circuit board with the widely used FR4 type dielectric. We used a very long pcb trace of $270 \mathrm{~cm}(106 ")$ to be able to achieve a high channel loss at the Nyquist frequency. The trace is single-ended. It is a microstrip with a characteristic impedance of $50 \Omega$. There are no vias, and at both ends there are SMA connectors.

Reflections were reduced to a minimum by choosing channels with well controlled characteristic impedances and terminating them with well matched resistors.

Chapter 4 describes how these channels are used to determine the performance of the equalizer. For this purpose, we needed to use a number of channels that are different in terms of physical configuration and skin effect / dielectric loss ratios. The lengths of the coaxial cables are chosen such that the equalizer can be tested at the highest speed and maximum loss compensation that it can handle. The PWM equalizer can typically handle up to $30 \mathrm{~dB}$ of loss at $5 \mathrm{~Gb} / \mathrm{s}$, as is shown later.

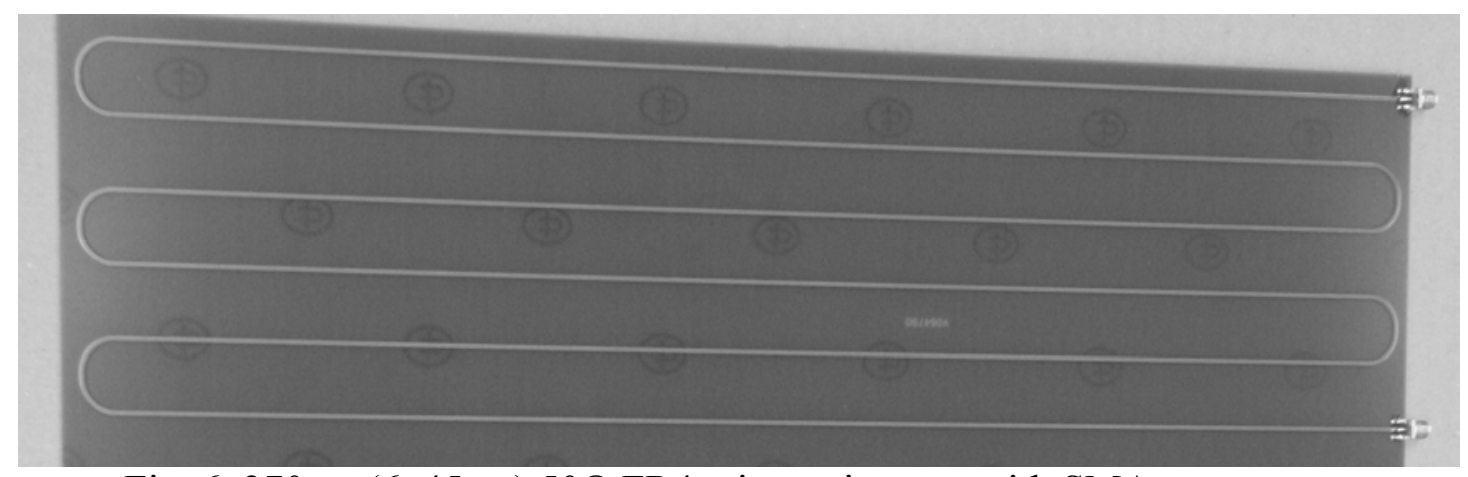

Fig. $6.270 \mathrm{~cm}(6 \mathrm{x} 45 \mathrm{~cm}) 50 \Omega$ FR4 microstrip trace with SMA connectors. 


\begin{tabular}{|c|c|c|c|c|c|}
\hline Name & (a) & (b) & (c) & (d) & $(\mathrm{e})$ \\
\hline Type & RG-58CU & Aircom+ & Aircell7 & $\begin{array}{c}\text { 10GBASE- } \\
\text { CX4 }\end{array}$ & $\begin{array}{c}\text { PCB } \\
\text { microstrip }\end{array}$ \\
\hline \multicolumn{6}{|c|}{ Physical parameters } \\
\hline Length & $25 \mathrm{~m}$ & $130 \mathrm{~m}$ & $80 \mathrm{~m}$ & $15 \mathrm{~m}$ & $270 \mathrm{~cm}$ \\
\hline Conduct. $(\sigma)$ & $5.810^{7}$ & $5.810^{7}$ & $5.810^{7}$ & $5.810^{7}$ & $5.810^{7}$ \\
\hline $\begin{array}{c}\text { Cable outer } \\
\text { diameter }\end{array}$ & $5.1 \mathrm{~mm}$ & $10.3 \mathrm{~mm}$ & $7.3 \mathrm{~mm}$ & $\begin{array}{c}10 \mathrm{~mm} \\
\text { (8 pairs) }\end{array}$ & $\mathrm{n} / \mathrm{a}$ \\
\hline $\begin{array}{l}\text { Radius inner } \\
\text { conductor }(a)\end{array}$ & $\begin{array}{l}0.45 \mathrm{~mm} \\
\text { (woven) }\end{array}$ & $\begin{array}{l}1.35 \mathrm{~mm} \\
\text { (solid) }\end{array}$ & $\begin{array}{l}0.93 \mathrm{~mm} \\
\text { (woven) }\end{array}$ & $\mathrm{n} / \mathrm{a}$ & $\mathrm{n} / \mathrm{a}$ \\
\hline $\begin{array}{l}\text { Radius outer } \\
\text { conductor }(b)\end{array}$ & $1.48 \mathrm{~mm}$ & $3.6 \mathrm{~mm}$ & $2.5 \mathrm{~mm}$ & $\mathrm{n} / \mathrm{a}$ & $\mathrm{n} / \mathrm{a}$ \\
\hline $\begin{array}{c}\text { Diameter of } \\
\text { cond. }(d)\end{array}$ & $\mathrm{n} / \mathrm{a}$ & $\mathrm{n} / \mathrm{a}$ & $\mathrm{n} / \mathrm{a}$ & $\begin{array}{c}0.51 \mathrm{~mm} \\
(24 \mathrm{AWG})\end{array}$ & $\mathrm{n} / \mathrm{a}$ \\
\hline $\begin{array}{c}\text { Dist. between } \\
\text { cond. }(D)\end{array}$ & $\mathrm{n} / \mathrm{a}$ & $\mathrm{n} / \mathrm{a}$ & $\mathrm{n} / \mathrm{a}$ & $0.8 \mathrm{~mm}$ & $\mathrm{n} / \mathrm{a}$ \\
\hline Width $(w)$ & $\mathrm{n} / \mathrm{a}$ & $\mathrm{n} / \mathrm{a}$ & $\mathrm{n} / \mathrm{a}$ & $\mathrm{n} / \mathrm{a}$ & $1.2 \mathrm{~mm}$ \\
\hline $\begin{array}{c}\text { Dielectric } \\
\text { distance }(d)\end{array}$ & $\mathrm{n} / \mathrm{a}$ & $\mathrm{n} / \mathrm{a}$ & $\mathrm{n} / \mathrm{a}$ & $\mathrm{n} / \mathrm{a}$ & $0.8 \mathrm{~mm}$ \\
\hline Thickness $(h)$ & $\mathrm{n} / \mathrm{a}$ & $\mathrm{n} / \mathrm{a}$ & $\mathrm{n} / \mathrm{a}$ & $\mathrm{n} / \mathrm{a}$ & 45um \\
\hline Dielectric & Polyethylene & Air & Foam & Foam & FR4 \\
\hline \multicolumn{6}{|c|}{ Measured parameters } \\
\hline $\begin{array}{l}\text { Loss } / 10 \mathrm{~m} \\
@ 2.5 \mathrm{GHz}\end{array}$ & $12.4 \mathrm{~dB}$ & $2.3 \mathrm{~dB}$ & $3.7 \mathrm{~dB}$ & $12.7 \mathrm{~dB}$ & $74.1 \mathrm{~dB}$ \\
\hline \multicolumn{6}{|c|}{ Calculated from physical parameters } \\
\hline$\lambda$ & $4.8010^{-5}$ & $1.6910^{-5}$ & $2.4510^{-5}$ & $1.6910^{-4}$ & $8.7410^{-5}$ \\
\hline$L_{e}$ & $2.3710^{-7}$ & $1.9610^{-7}$ & $1.9910^{-7}$ & $4.0910^{-7}$ & $3.1310^{-7}$ \\
\hline \multicolumn{6}{|c|}{ Model fit parameters } \\
\hline$\varepsilon_{r}^{\prime} \infty$ & 2.6 & 1.4 & 1.5 & 2.1 & 4.0 \\
\hline$\Delta \varepsilon_{r}^{\prime}$ & $8110^{-3}$ & $4.510^{-3}$ & $7.910^{-3}$ & $2110^{-3}$ & 1.5 \\
\hline$m_{1}$ & 1.7 & 1.5 & 3.8 & 3.3 & 1.1 \\
\hline$m_{2}$ & 14 & 14 & 14 & 14 & 14 \\
\hline$\tau_{1}$ & $1.0 \mathrm{~ns}$ & $1.3 \mathrm{~ns}$ & $1.2 \mathrm{~ns}$ & $0.63 \mathrm{~ns}$ & $0.5 \mathrm{~ns}$ \\
\hline
\end{tabular}

Table 1. Channel parameters. 


\subsubsection{Transient behavior}

To obtain a first impression of their transient behavior, the measured response of the four cables and the PCB to a PAM random sequence of bits is given. See Figs. 7-11 below.

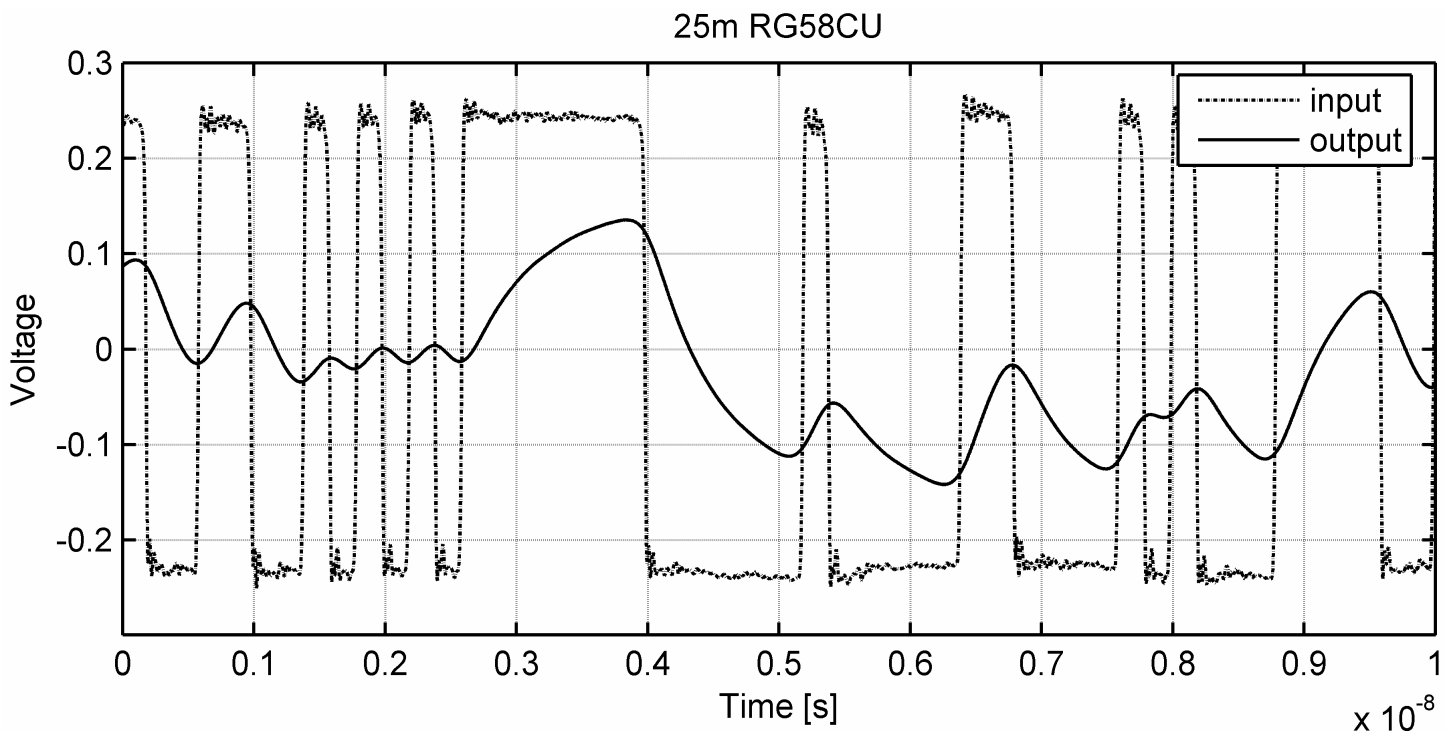

Fig. 7. Measured response of $25 \mathrm{~m}$ RG58CU coaxial cable (a).

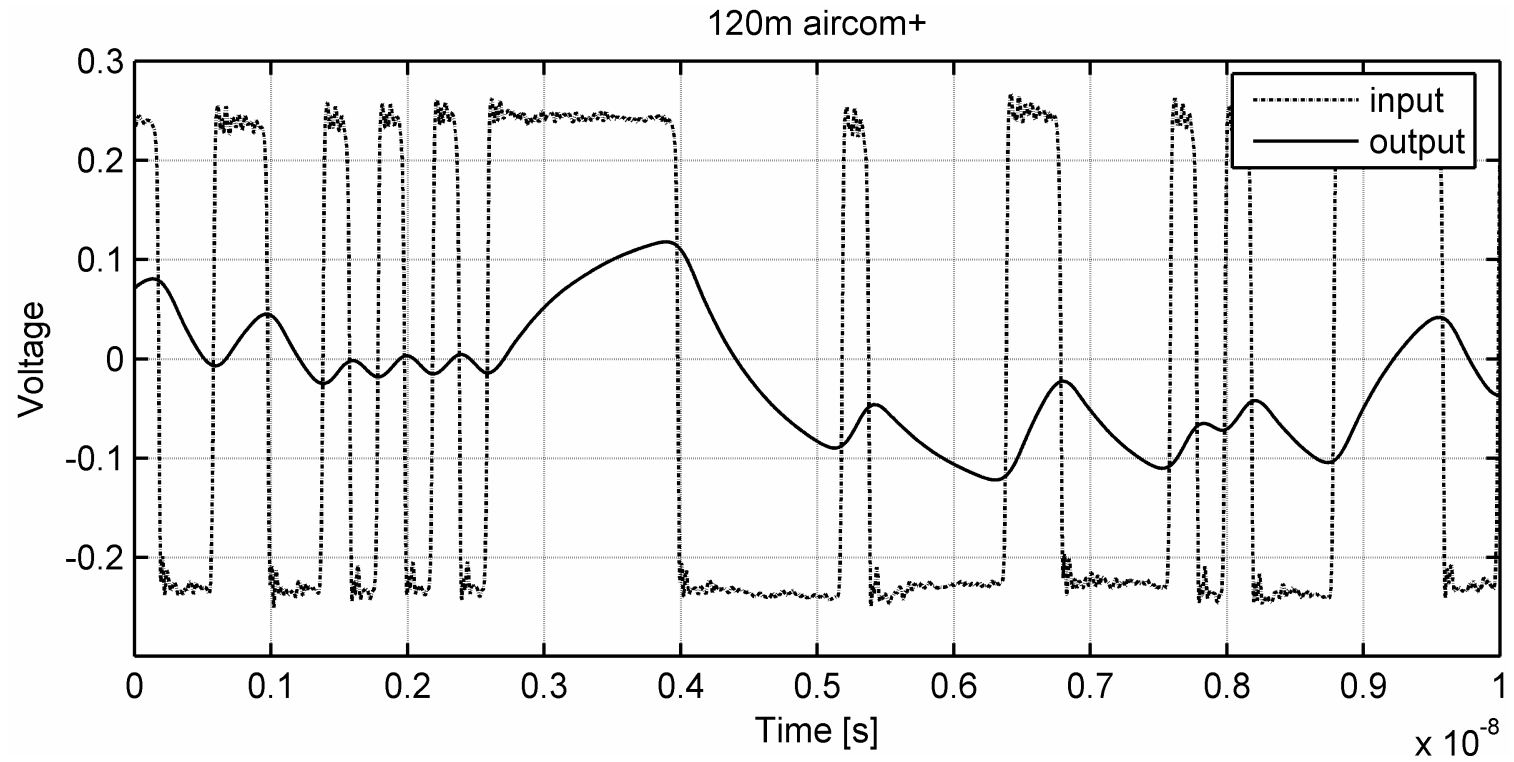

Fig. 8. Measured response of $120 \mathrm{~m}$ Aircom + coaxial cable (b). 


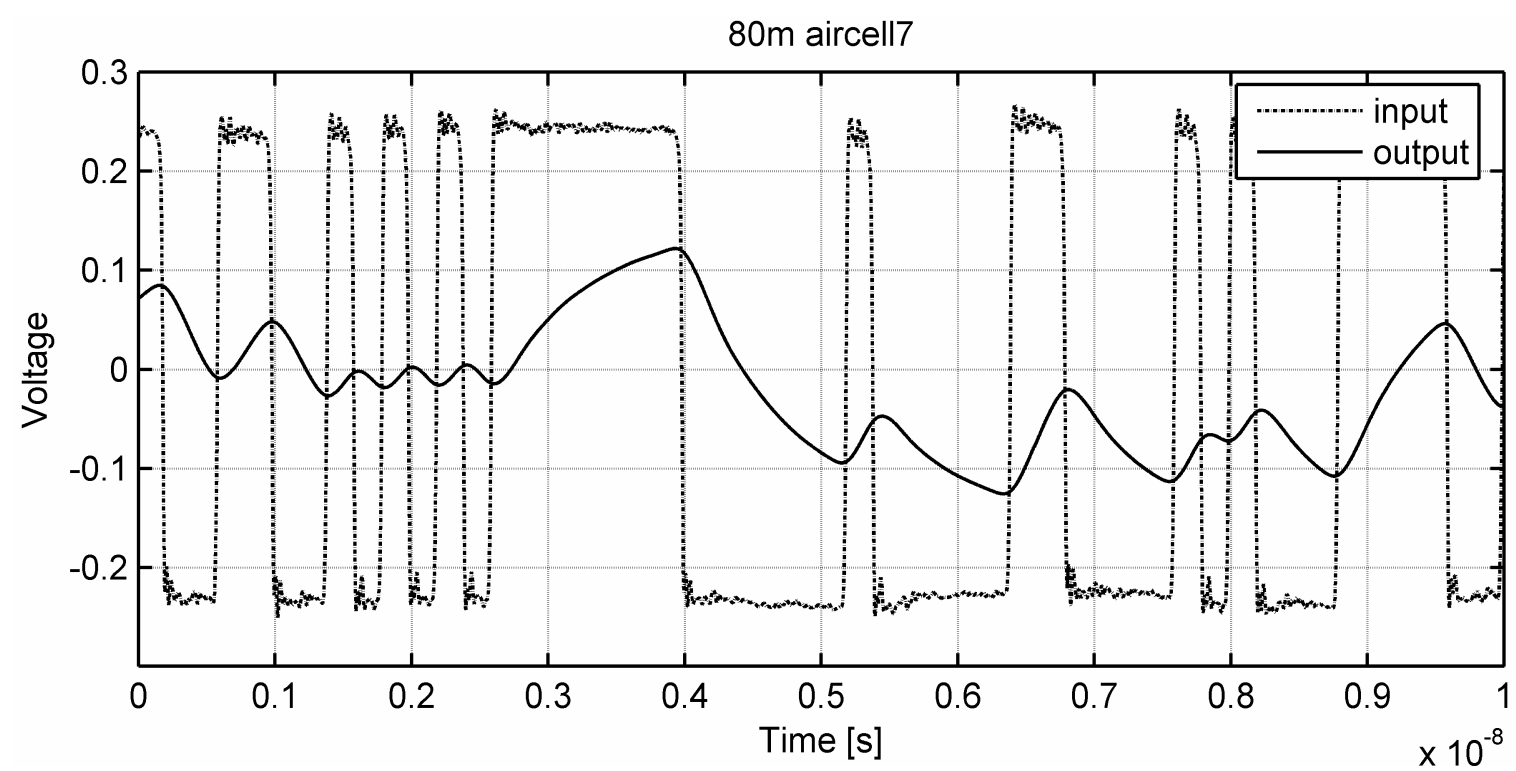

Fig. 9. Measured response of $80 \mathrm{~m}$ Aircell7 coaxial cable (c).

15m 10GBASE-CX4

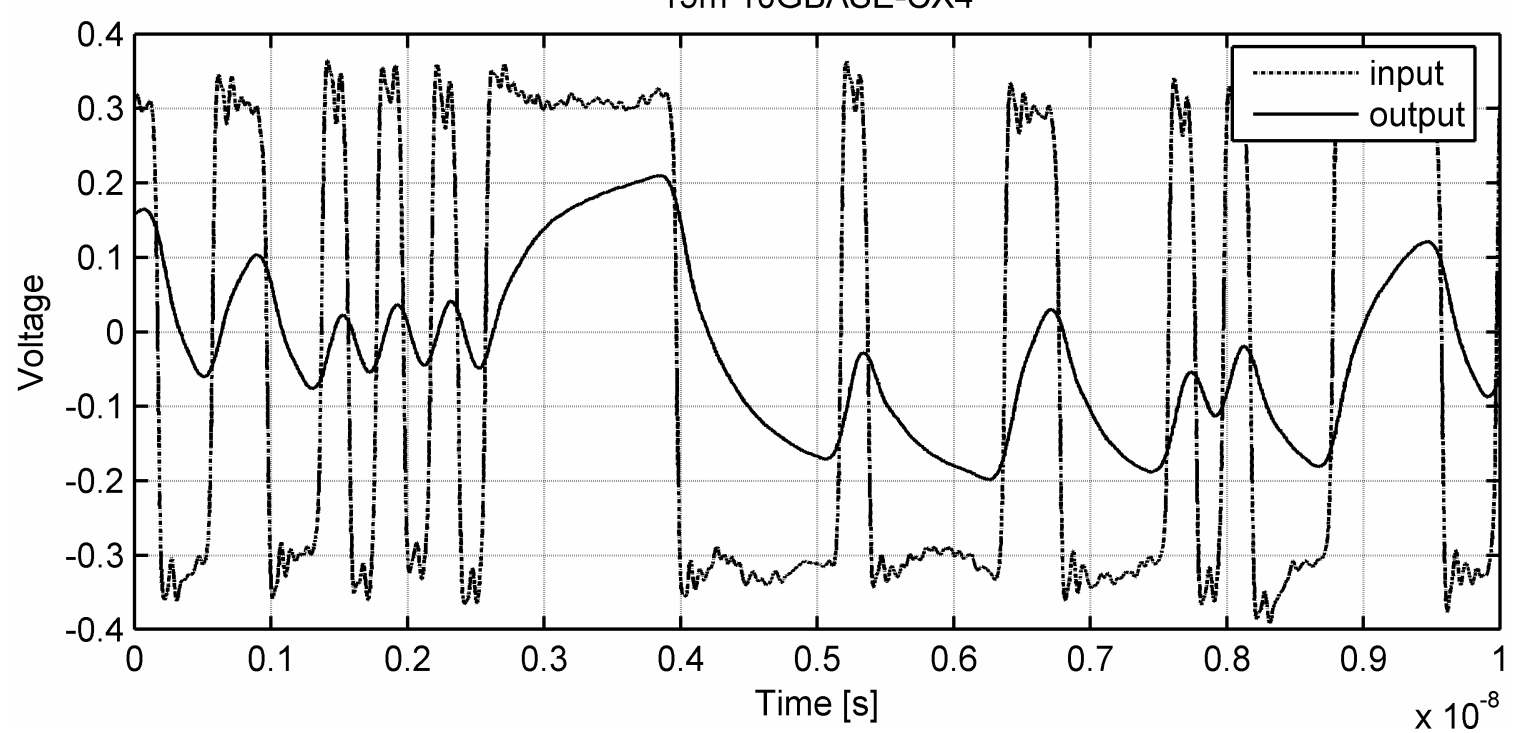

Fig. 10. Measured response of 15m 10GBASE-CX4 differential cable (d).

$270 \mathrm{~cm}$ FR4 pcb

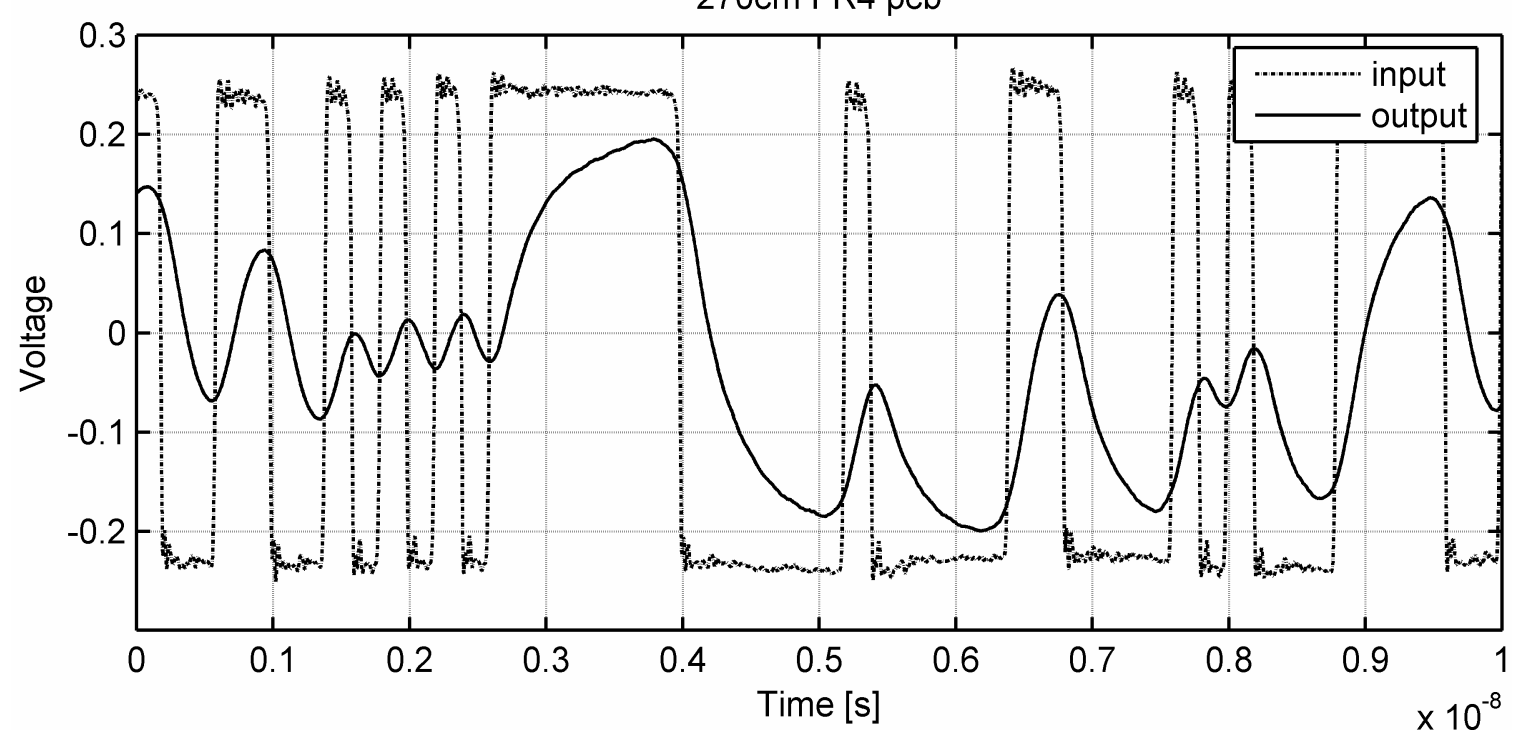

Fig. 11. Measured response of $270 \mathrm{~cm}$ FR4 pcb trace (e). 
In all cases, heavy ISI can be seen in the measurements. The amplitude of the output is higher for the twisted pair and the PCB channels, because of their lower losses, as is confirmed later by the $S_{21}$ measurements. Furthermore, a slightly higher ripple can be seen in the PCB measurements. This is due to the better shielding of the cables.

\subsubsection{Measurements - model match}

A comparison is made between the measurements and the model using the three-step methodology described below.

(1) A measurement of the $S_{21}$ (magnitude of the transfer function) of the channel is made using a network analyzer. The magnitude of the theoretical RLGC transfer function is fitted to it by adjusting the model parameters. Measurements and model fit are plotted in a figure. Using the equations from subsection 2.3.3, the skin loss and dielectric loss are calculated separately and also plotted.

(2) The step response of the cables is measured using time domain transmissometry (TDT). The impulse response is obtained by differentiation and (low-pass) noise filtering. This impulse response is compared with two theoretical impulse responses. The first is the inverse Fourier transform of the RLGC transfer function, used in step (1) above, calculated numerically. The second is the simple analytical skin effect impulse response given in subsection 2.4.1.

(3) Finally, the measured impulse response is Fourier transformed to obtain the frequency domain magnitude transfer. This is again compared to the frequency domain measurements in step (1).

\subsubsection{1. $S_{21}$ fit}

In Figs. 12-16, the measured and modeled magnitudes of the transfer function $S_{21}$ for the cables are shown, as described in step (1). The modeled contribution of conductor losses and dielectric losses is shown separately, using the equations from subsection 2.3.3. The measured and Fourier transformed impulse responses, as described in step (3), are also shown in this figure.

The legend for Figs. 12-16 is as follows:

- 'model': $\quad$ magnitude of Eq. 1,

- 'model (skin)': $\quad$ Eq. 39,

- 'model (diel)': $\quad$ Eq. 38 ,

- 'measured (NA)': measured using network analyzer,

- 'measured (FT ht)': measured using TDT and then Fourier transformed (see also subsection 2.5.3.2).

See Table 1 for the parameters that were used to fit the model. The optimization algorithm that was used for the model parameter fitting is Particle Swarm Optimization (PSO) [Kennedy]. The model parameters $\varepsilon_{r}{ }^{\prime} \infty, \Delta \varepsilon_{r}$ ' and $m_{1}$ for the dielectric loss were optimized to provide a model fit, while $m_{2}$ was fixed at 14 and the skin loss was fixed using the well known copper conductivity $\sigma=5.810^{7} \mathrm{~S} / \mathrm{m}$. When parameter $\varepsilon_{r}{ }^{\prime} \infty$ is averaged over the whole frequency range, it comes very close to the commonly known values for the relative dielectric permittivity $\varepsilon_{r}$. These values are 2.25 for polyethylene in RG58CU, 1.4 for foam/air dielectric in Aircell7 and Aircom+, and 4.9 for FR4. 
The dielectric loss of the PCB trace is lower than expected. It was designed for $30 \mathrm{~dB}$ loss at $2.5 \mathrm{GHz}$, using a loss tangent of 0.025 . The measured loss is $21 \mathrm{~dB}$ instead, so the dielectric loss is lower in this specific board. (In Chapter 4 the total channel loss is $25 \mathrm{~dB}$ because a $1.75 \mathrm{~m}$ coaxial cable and a bias tee are also used for connecting to the chip.)

Figs. 12-16 show the measurements and model fits in the frequency domain for $25 \mathrm{~m}$ RG-58CU, 80m Aircell7, 130m Aircom+, 15m 10GBASE-CX4, and 270cm FR4 microstrip trace respectively. The measured network analyzer data and the modeled transfer function only differ significantly for RG-58CU above $2.5 \mathrm{GHz}$. It is most likely that its $\mathrm{BNC}$ connectors cause impedance mismatches at those frequencies.

The Fourier transformed time domain measurements - from step (3), shown in the figures as 'FT ht' - differ slightly from the network analyzer data. A possible cause for this is that this result is calculated using the response to a non-ideal step, with finite steepness. For the PCB and the twisted pair (10GBASE-CX4), the difference is somewhat larger. It is most probably caused by the short pieces of coaxial cable needed to connect them to the TDT equipment. From the frequency domain measurements with the network analyzer, the effect of these short cables was removed by calibration, but from the TDT measurements it was not. However, in all cases the difference is not greater than $1-2 \mathrm{~dB}$ at $2.5 \mathrm{GHz}$.

Cable (b) has the highest ratio of skin loss to dielectric loss, because of its air dielectric. Cable (a) has the crossing point between skin loss and dielectric loss at the lowest frequency of the copper cables, at $2.2 \mathrm{GHz}$. Losses from the printed circuit board trace (e) are clearly dominated by dielectric losses.

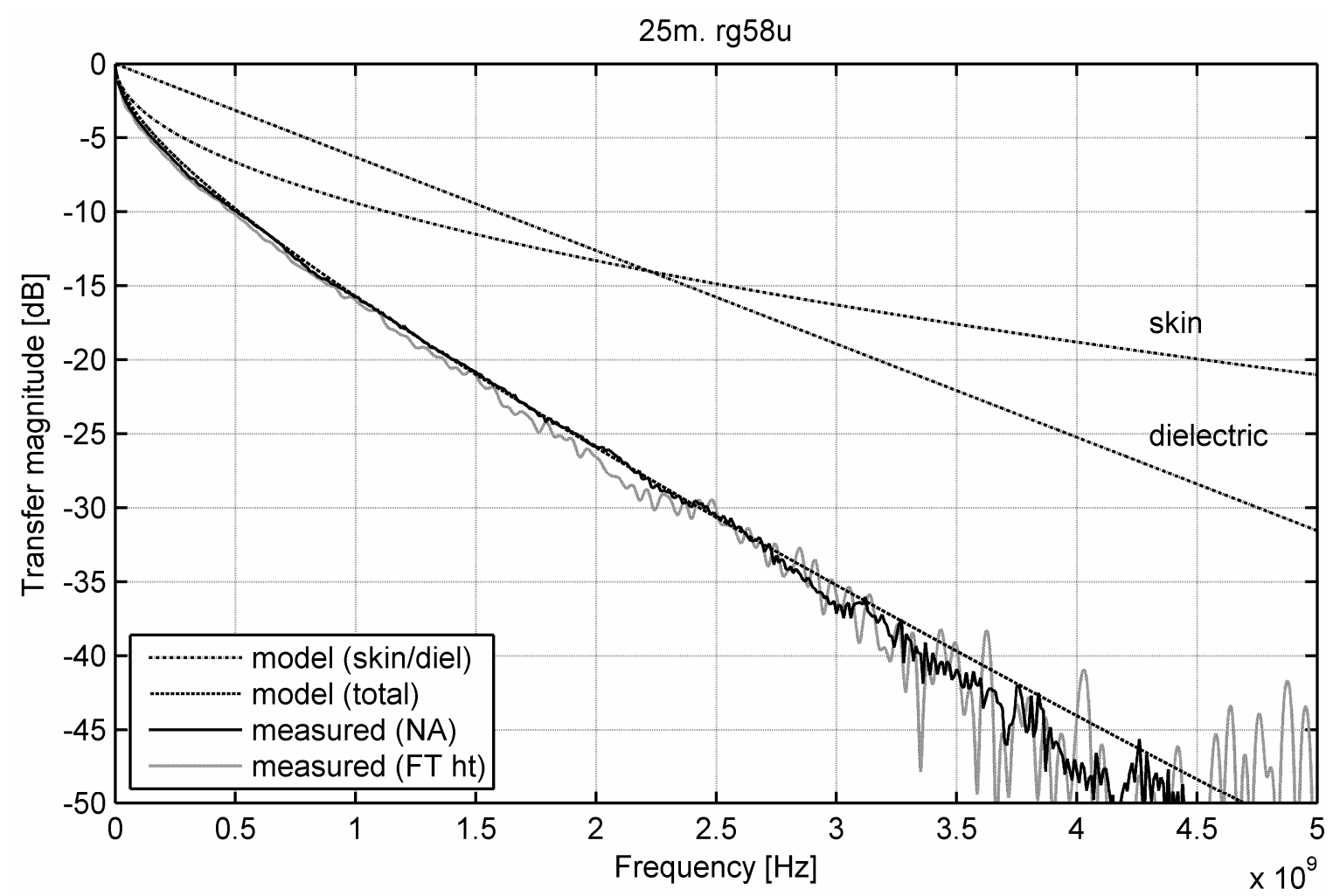

Fig. 12. Measured $S_{21}$, Fourier transform of measured impulse response, and model fit for 25m RG-58CU cable (a). 


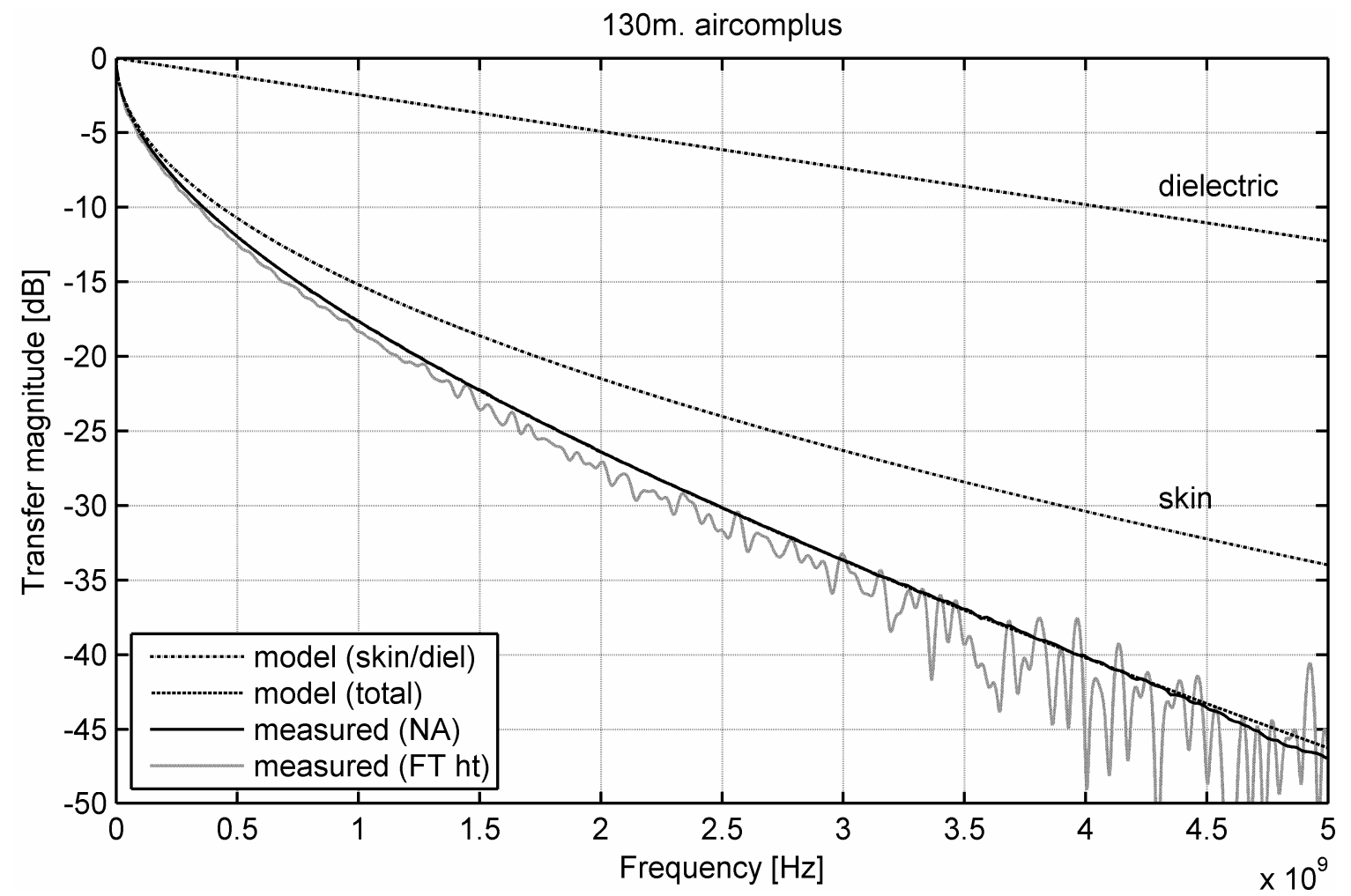

Fig. 13. Measured $S_{21}$, Fourier transform of measured impulse response, and model fit for 130m Aircom+ cable (b).

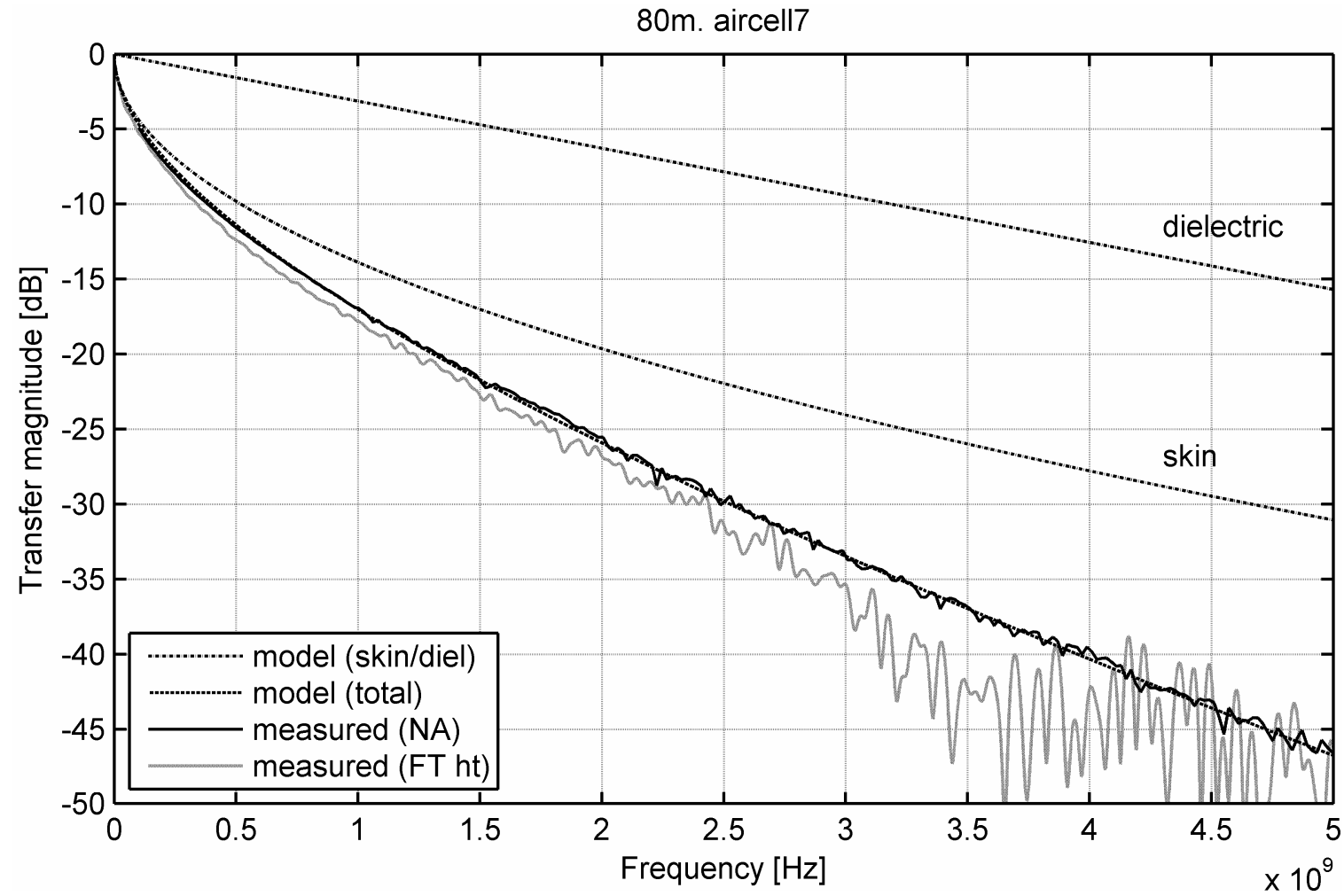

Fig. 14. Measured $S_{21}$, Fourier transform of measured impulse response, and model fit for 80m Aircell7 cable (c). 
15m. 10GBASE-CX4

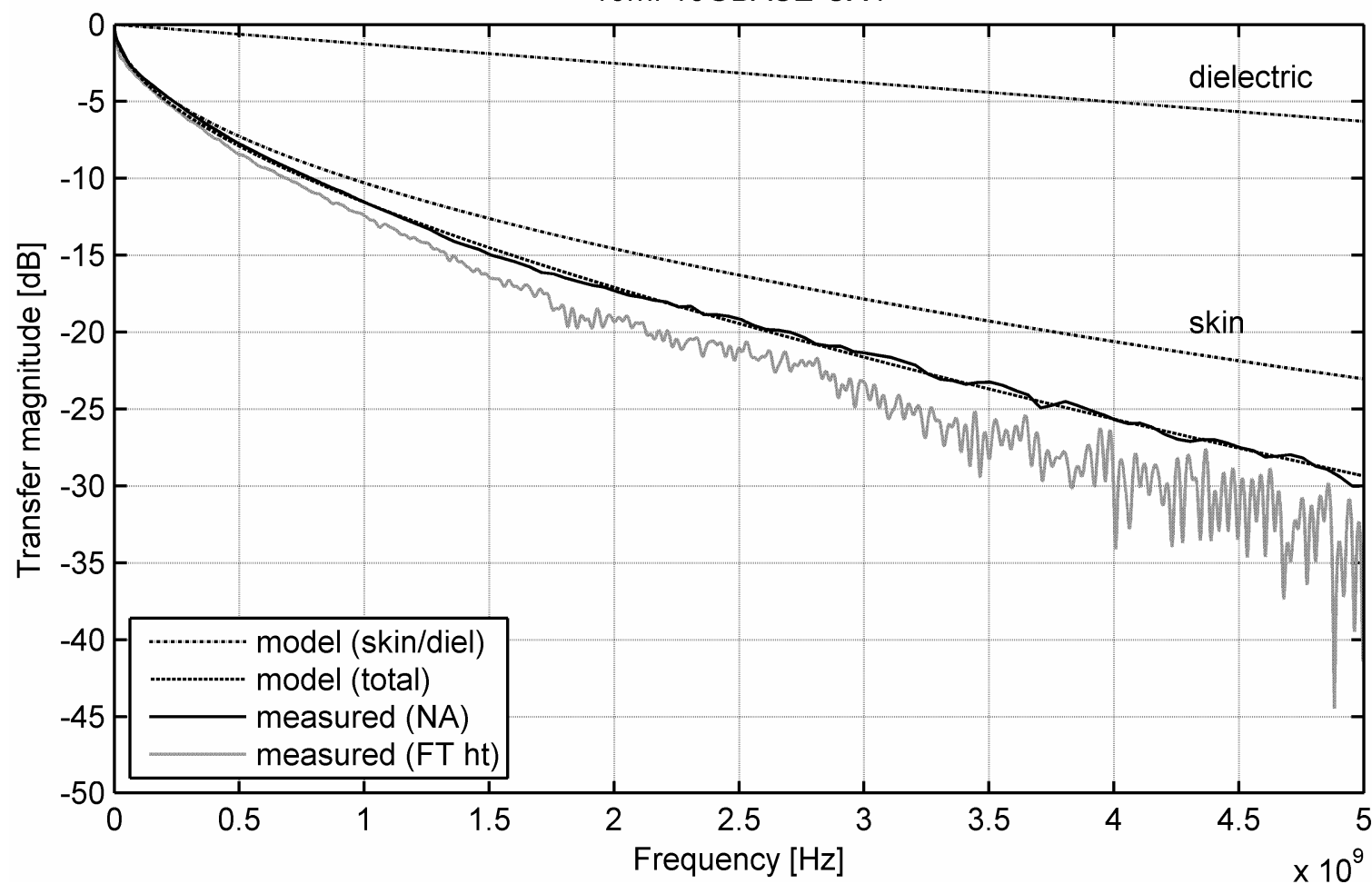

Fig. 15. Measured $S_{21}$, Fourier transform of measured impulse response, and model fit for 15m 10GBASE-CX4 24AWG (d). (Measured: $S_{d d 21}$.)

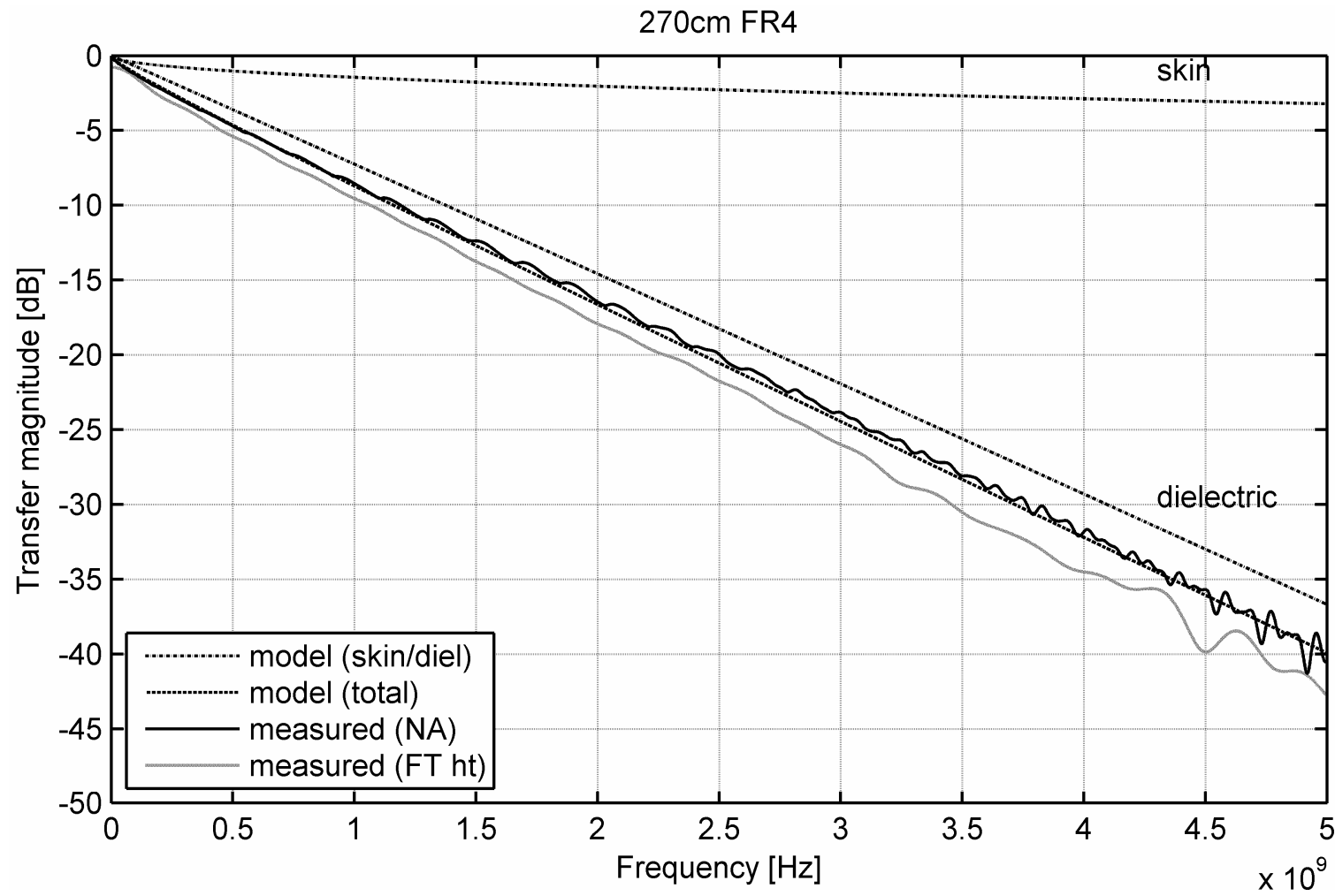

Fig. 16. Measured $S_{21}$, Fourier transform of measured impulse response, and model fit for $270 \mathrm{~cm}$ long microstrip on FR4 printed circuit board (e). 


\subsubsection{Impulse response fit}

For step (2) we show the time domain measurements and compare them to the models. A 40ps-risetime, 200ps step was used to measure the step responses. The measured step response was then differentiated to obtain the impulse response. To remove the highfrequency noise on this impulse response, a linear-phase equiripple low-pass FIR filter was used with a passband of $10 \mathrm{GHz}(0.01 \mathrm{~dB}$ ripple), and $20 \mathrm{~dB}$ attenuation in the stopband (starting at $15 \mathrm{GHz}$ ). Figs. 17-21 show the results.

The legend for Figs. 17-21 is as follows:

- 'measured': $\quad$ impulse response from differentiated, noise filtered, step response,

- 'model (total)': numerically calculated inverse Fourier transform of Eq. 1 (of which the magnitude was fitted to the measured $S_{21}$ in step (1); includes both skin effect and dielectric loss.

- 'model (skin)': $\quad$ Theoretical impulse response for isolated skin effect (subsection 2.4.1), calculated using parameter $\tau_{l}$ in Table 1 .

While most of the modeled impulse responses fit well with those from measurements, there is a small difference for both the 10GBASE-CX4 cable and the FR4 trace. As explained in the previous subsection, a likely cause is that they needed to be connected to the measurement equipment using short coaxial wires (20 cm long). The effect of these short cables was removed by calibration from the network analyzer measurements, but not from the step response measurements.

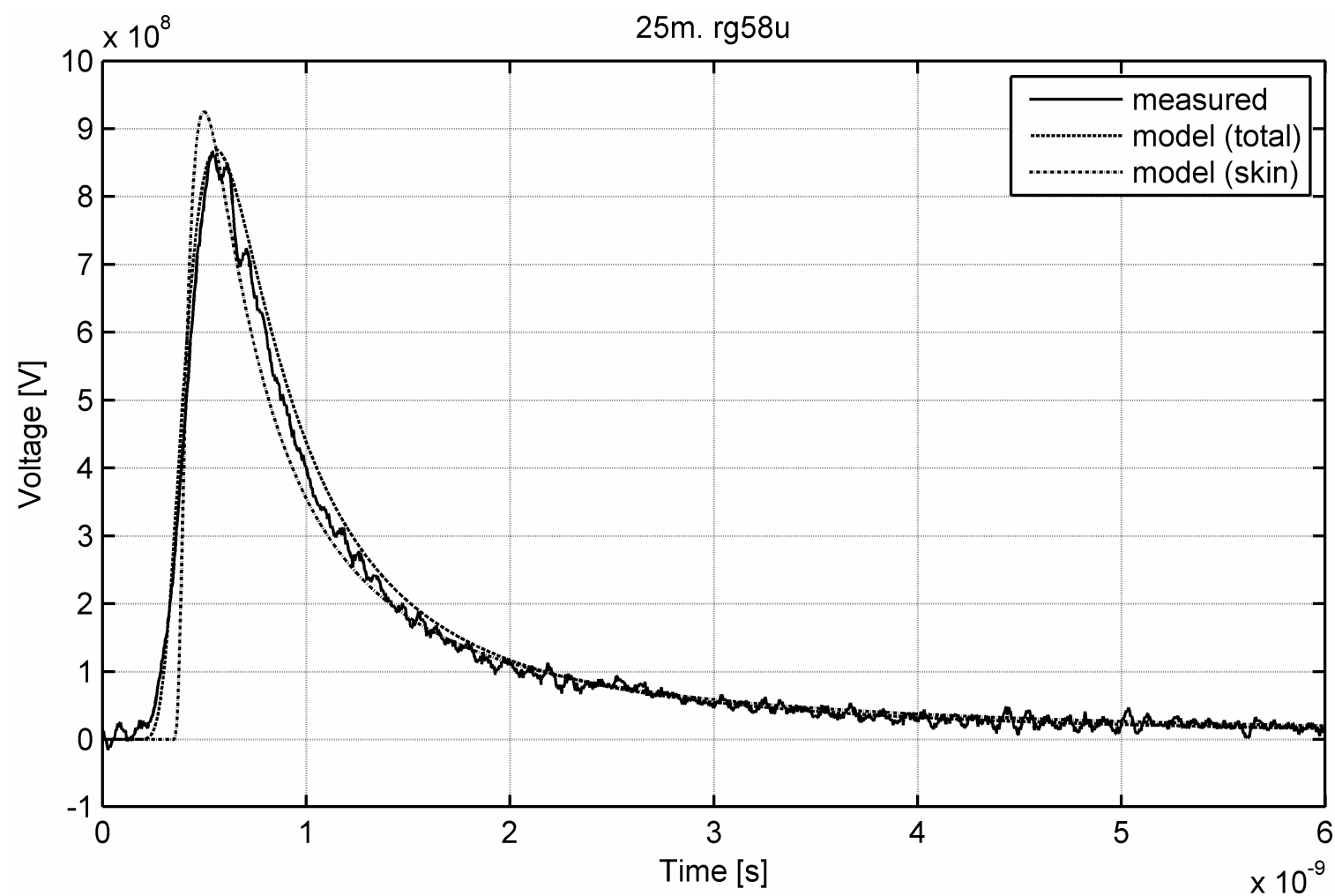

Fig. 17. Impulse response from measurements and model fit for $25 \mathrm{~m}$ RG-58CU cable (a). 


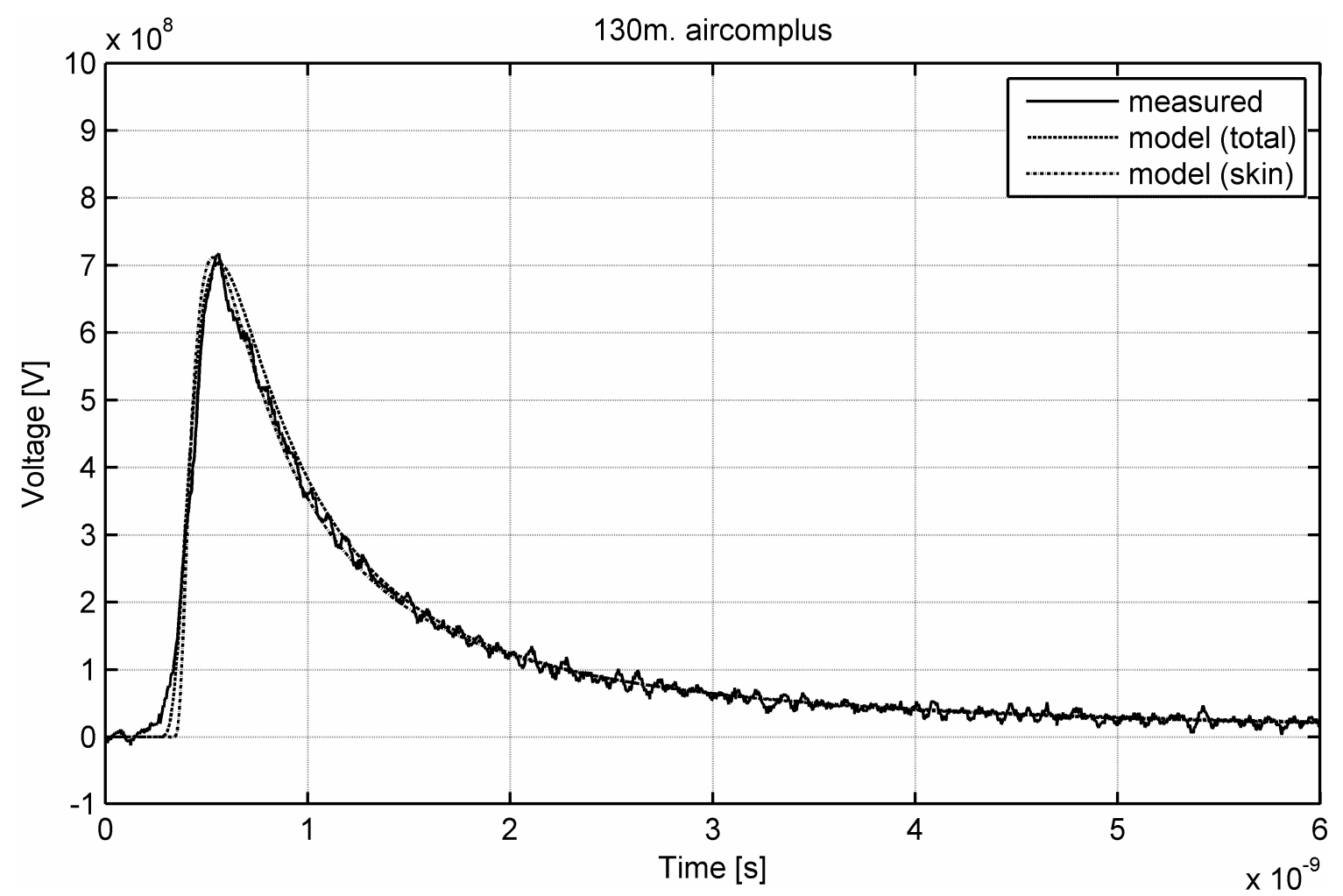

Fig. 18. Impulse response from measurements and model fit for $130 \mathrm{~m}$ Aircom + cable (b).

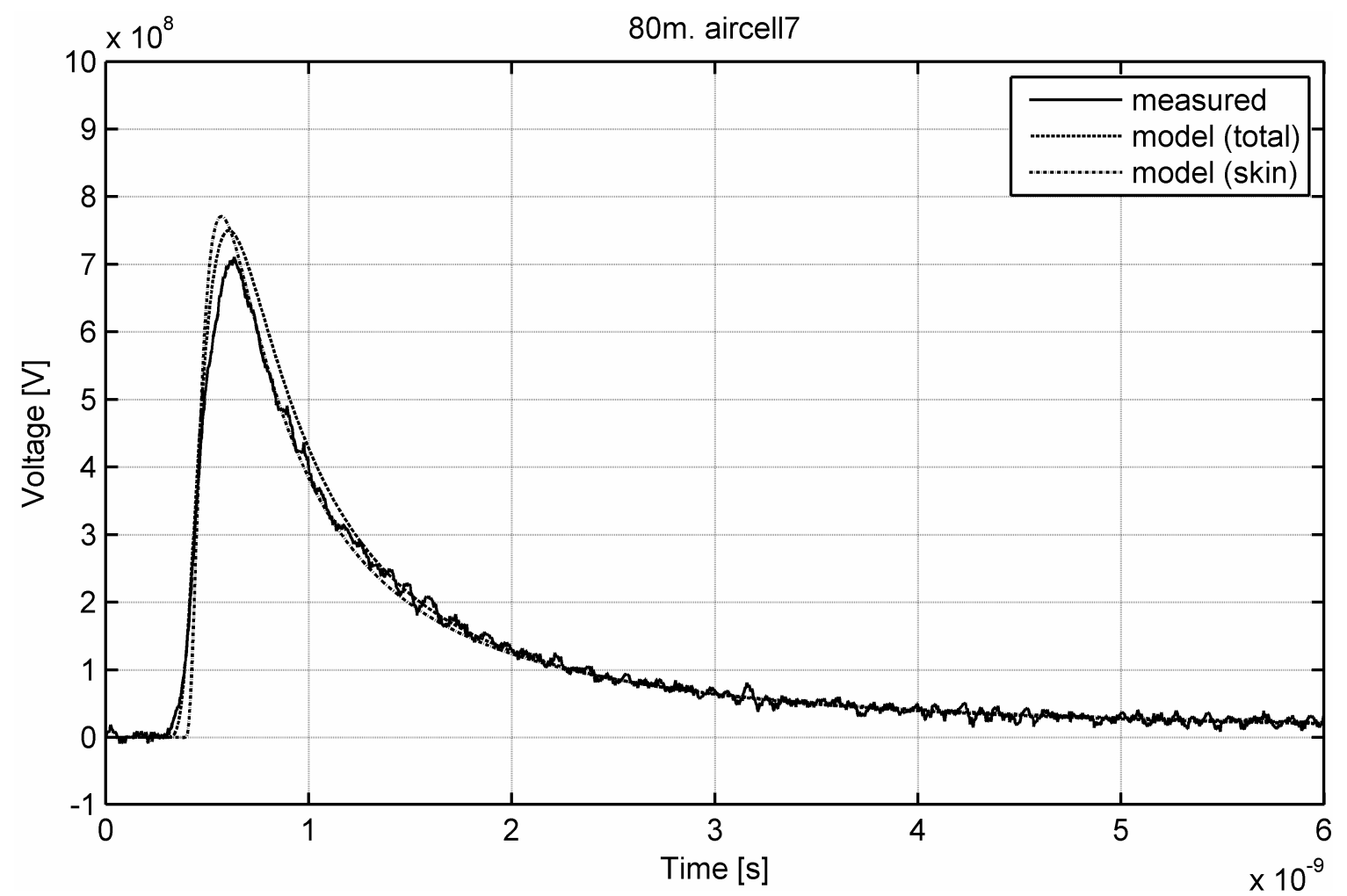

Fig. 19. Impulse response from measurements and model fit for $80 \mathrm{~m}$ Aircell7 cable (c). 


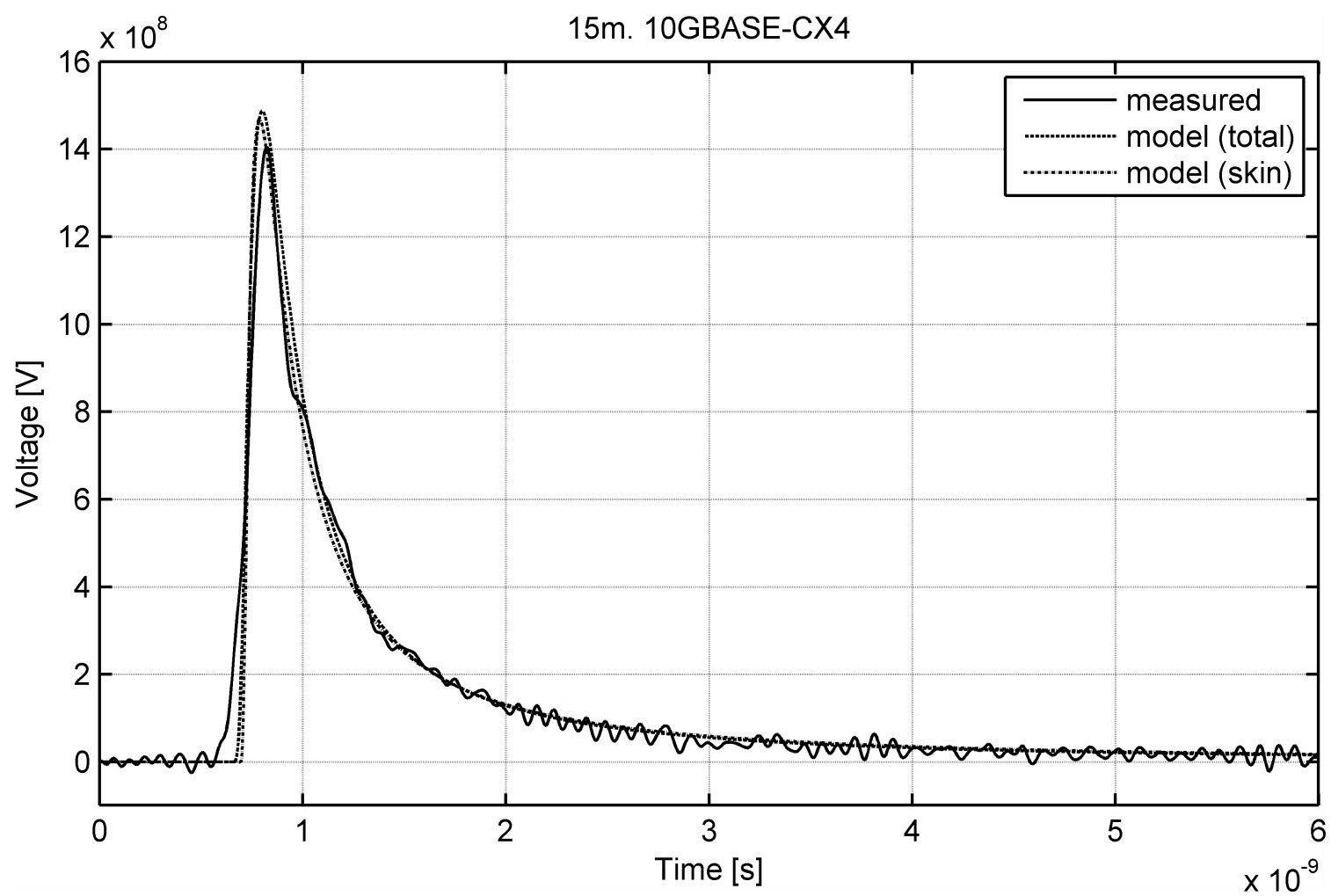

Fig. 20. (Differential) impulse response from measurements and model fit for 15m 10GBASE-CX4 24AWG (d).

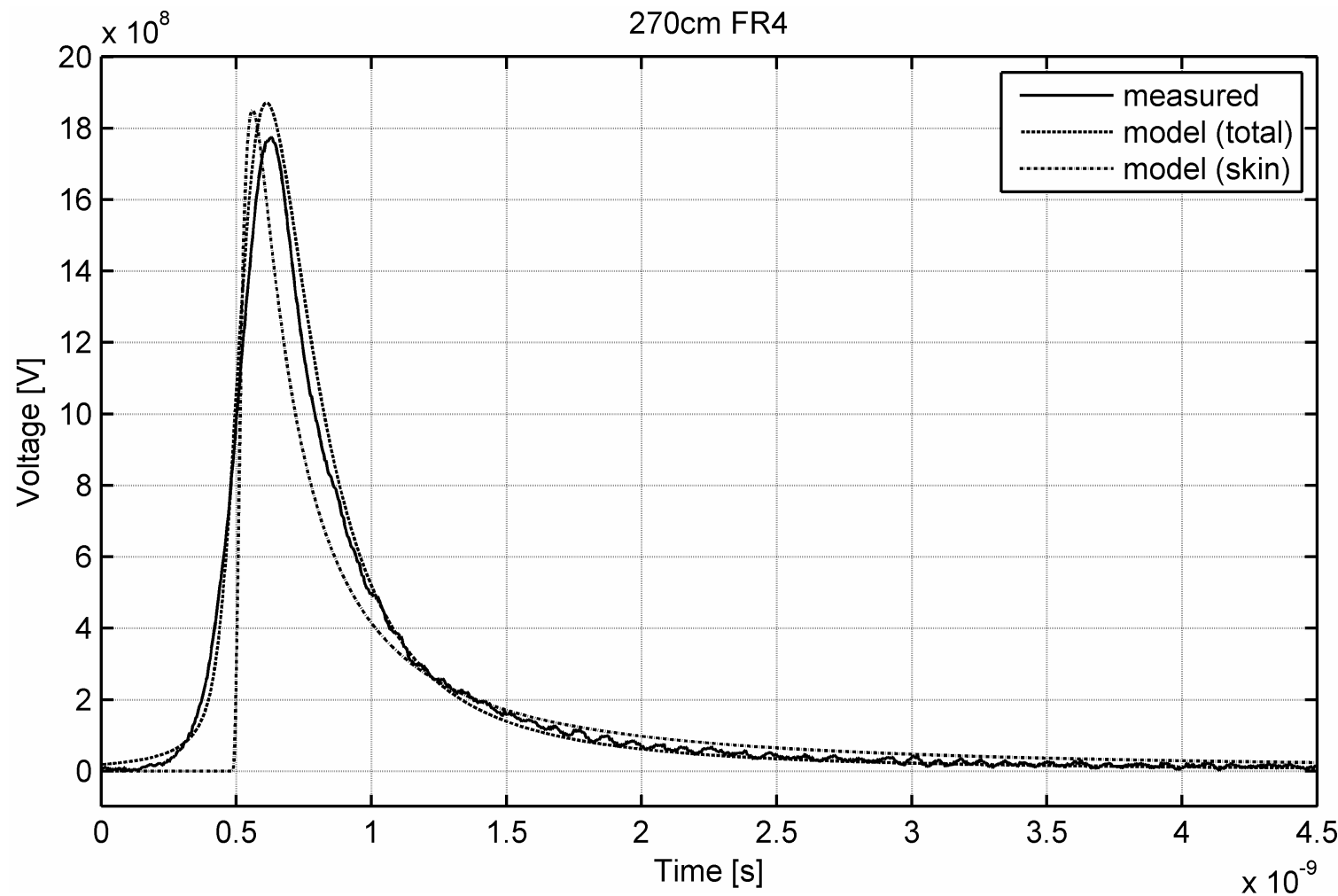

Fig. 21. Impulse response from measurements and model fit for $270 \mathrm{~cm}$ long microstrip on FR4 printed circuit board (e). 


\subsection{Conclusions}

A practical model for copper channels is described, modeling both skin effect and dielectric loss. Special attention was given to causality, to provide an accurate impulse response for use in high speed transient simulations.

The well known formulas for skin effect were combined with a new formula for dielectric loss in FR4, published only in 2001. This formula for dielectric loss is shown in this chapter to be not only useful and accurate for modeling the dielectric loss on printed circuit boards, but also for modeling cables. Both the complex skin-effect impedance and the complex dielectric impedance comply with the Kramers-Kronig relations. Therefore, an inverse Fourier transform of the transfer function yields a causal time domain response.

Measurements show a good model fit in the time domain with coaxial copper cables, a twisted pair cable, and a printed circuit board trace. We can conveniently use the modeled impulse response for accurate transient simulations of high-speed communication systems. 


\section{Chapter 3}

\section{Effect of timing non-idealities on an analog multicarrier system}

\subsection{Introduction}

As discussed in Chapter 1, many publications dealing with data-communication over short length copper wires, e.g. [Farjad-Rad], refer to Pulse Amplitude Modulation (PAM). This modulation method is well suited to a channel with a monotonously decreasing magnitude of the transfer function. However, in some circumstances, for example communication over lowquality PCBs with many impedance discontinuities such as vias, some spectral nulls can be present in the magnitude transfer function. In such cases a linear zero-forcing equalizer in the PAM systems would amplify the noise in the spectral null areas by a large factor [Bingham]. Then orthogonal frequency division multiplexing (OFDM) can be a convenient modulation method, because the signal power distribution can be better tailored to the spectrum. This is also advantageous for channels that suffer from narrowband ingress noise. In frequency ranges with the best SNR we can apply more signal power, while in the frequency ranges with lower SNR less signal power is spent (the 'water-pouring' principle) [Bingham]. Examples of OFDM applications include wireless LANs for data connections over radio channels, and (A)DSL for data connections over the plain old telephone system (POTS).

For the above reasons, some publications suggest the use of OFDM for communication over (for example) backplane PCBs with deep spectral nulls in their transfer function [Amirkhany-1]. However, due to the very high bandwidth (in the GHz range) of such links such a system can not be implemented in a similar way to that in the above two examples, in which the common implementation termed 'discrete multitone' (DMT) is used. In a DMT implementation, A-to-D converters and D-to-A converters convert from the analog to the digital domain and back, and most signal operations, like the fast Fourier transform (FFT), are carried out in the digital domain using digital signal processors.

As an alternative, in this chapter we look at the feasibility of an 'analog' OFDM system for data-transmission at gigabit rates $(\sim 10 \mathrm{~Gb} / \mathrm{s})$ over backplanes. Such a system consists of analog mixers and analog integrate-and-dump blocks to first separate (demodulate) the OFDM subcarriers and then digitize them. This chapter is based on our existing publications [Schrader-2] and [Schrader-3].

This chapter aims to improve the understanding of the impact of timing non-idealities on such an 'analog' multi-tone system. First, in section 3.2, we briefly summarize the main principles underlying OFDM and its inherent advantage in terms of channel equalization. Section 3.3 then describes the proposed analog OFDM system. Next, section 3.4 characterizes the jitter that disturbs the system. We outline the different types of inter-carrier interference in section 3.5. Next, analytical calculations (section 3.6) and statistical simulations (section 3.7) are presented that give insight into the system-level trade-offs and possibilities of an analog OFDM system. More specifically, the effect of jitter and duty-cycle deviations on such a 
system is analyzed. We calculate SNRs and error rates for a number of different values for the RMS jitter, and for a number of different duty-cycle deviations. A bit rate limit is calculated and conclusions are drawn about the feasibility of such a system. In section 3.8 the results are compared with work from the literature. Finally conclusions are drawn in section 3.9.

\subsection{OFDM principles}

In OFDM, the transmitted data is modulated on several orthogonal subcarriers/tones. In order to avoid interference, these carriers have to comply with the orthogonality constraint, which is defined as (normalized) [Bingham]:

$$
\frac{1}{T_{o p}} \int_{0}^{T o p} c_{i}(t) \cdot c_{j}(t) d t=\delta_{i j}, \quad \delta_{i j}= \begin{cases}1, & i=j, \\ 0, & i \neq j,\end{cases}
$$

where $c_{i, j}(t)$ are the carriers and $T_{o p}$ is the length of the receiver integration period ('orthogonality period'). Candidates for subcarrier/tone frequencies $f_{c}$ are harmonic frequencies $n / T_{o p}$ with integer $n$. Integration over exactly $T_{o p}$ delivers perfectly orthogonal carriers.

The total bit rate $b_{t o t}$ of such a system will be:

$$
b_{\text {tot }}=\frac{2}{T_{s}} \sum_{i=1}^{N_{t}}{ }^{2} \log \left(N_{l, i}\right),
$$

where $N_{l, i}$ is the number of DAC/ADC levels used on the $i^{\text {th }}$ tone (where each tone is modulated with both an in-phase- and quadrature component - hence the factor two), $N_{t}$ the number of tone frequencies in the symbol and $T_{s}$ the total symbol length, including guard time $T_{g t}$. So $T_{s}=T_{o p}+T_{g t}$.

OFDM has an inherent advantage in respect of equalization, as compared to linear equalization in e.g. a PAM system. In OFDM, the symbol rate is quite low compared to the channel bandwidth, due to the high number of subcarriers. Each subcarrier has a low bit rate. This, together with the guard time (cyclic prefix), simplifies the implementation of channel equalization [Bingham]. In OFDM, a Fourier transform is taken of the signal, the signal spectrum is obtained, and the channel transfer function is assumed to be flat within one single OFDM subcarrier. Channel equalization is then achieved through one complex multiplication of an equalization vector with the spectrum. The only constraint on the channel impulse response is that it fits within the cyclic prefix. To shorten the necessary cyclic prefix, an optional simple 'impulse response shortening' time-domain equalizer can also be added.

\subsection{Practical proposal for 'analog OFDM' system architecture}

In common Discrete Multi-Tone (DMT) implementations, the ADCs and DACs and the complex digital processing put limits on the bandwidth. In general, these systems are implemented using DSP techniques, but this is currently infeasible for a bandwidth in the gigahertz range. We introduce an architecture that solves some of these problems. A possible way to overcome the bandwidth limitation is to use analog multipliers and integrate-anddump blocks. This combination effectively performs a Fourier transform in the analog domain. 


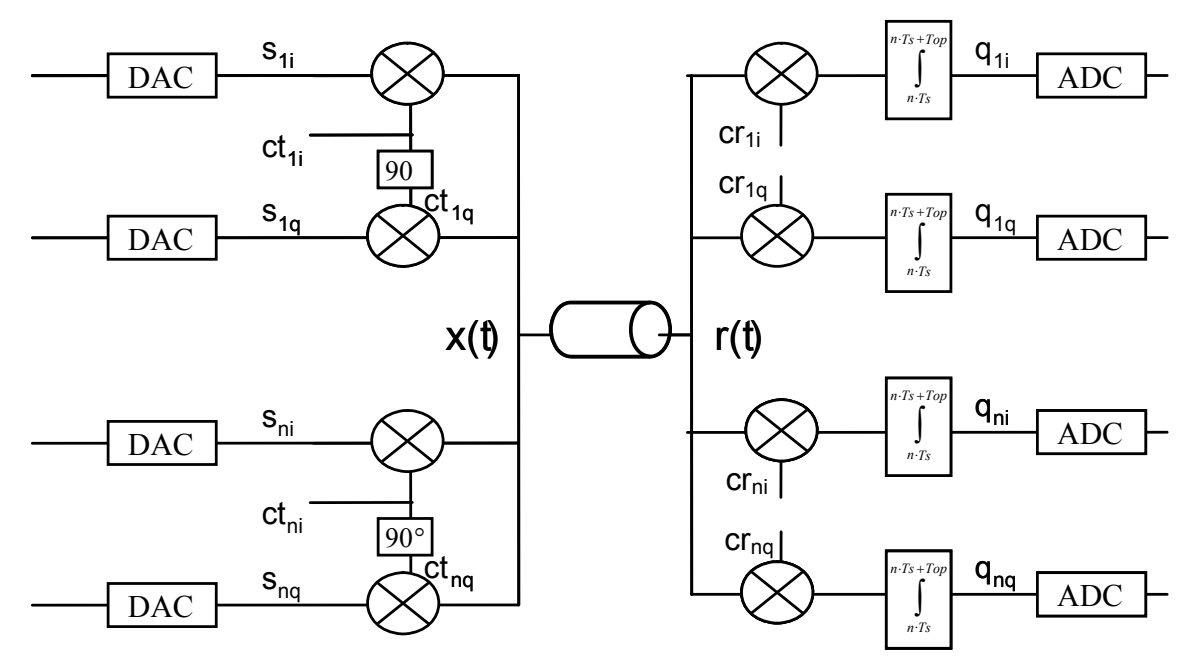

Fig. 1. Multi-carrier system using analog correlation

The multi-carrier communication system architecture that we study is shown in Fig. 1. On the left, the transmitter is shown and on the right, the receiver is shown. We use (passive or active) mixers for multiplication of the data streams with the carrier signals. For the integration over the symbol period, an integrate-and-dump block is used. ADCs and DACs can be added to provide more levels - and increase spectral efficiency. One architectural advantage now becomes apparent: parallelization is used for the converters and integrate-anddump blocks, which relaxes bandwidth requirements.

In-phase and quadrature components of the subcarriers can be generated using a voltagecontrolled oscillator. The multiple phases coming from the oscillator can also be used to control the integrate-and-dump blocks. Of course, a clock-and-data-recovery (CDR) block also needs be included in the receiver. This architecture puts a number of constraints onto the carrier waveforms that we can use. In an implementation with simple switching mixers, a sine wave on the local oscillator port will generate a square wave on the output. This will produce harmonics that fall onto other tone frequencies, creating unusable areas in the spectrum. Because of the abovementioned problems, it is likely that such a system would perform best when only a low number of subcarriers is used. A solution to the problem could be the use of harmonic rejection mixers [Weldon].

The signals in Fig. 1 are defined as follows. The bit streams $s_{n i}(t)$ and $s_{n q}(t)$, chosen from $\left\{-A_{\max }, A_{\max }\right\}$, are modulated onto the (in-phase and quadrature) carrier signals $c t_{n i}(t)$ and $c t_{n q}(t)$ and summed up, resulting in the sum signal $x(t)$ which is put onto the transmission line. At the receiver side the received signal $r(t)$ is demodulated using a 'correlator receiver' consisting of a multiplication with locally generated (in-phase and quadrature) carrier signals $c r_{n i}(t)$ and $c r_{n q}(t)$ and an integrate-and-dump operation. The recovered (soft-)bit streams are $q_{n i}(t)$ and $q_{n q}(t)$. The transmitted signal $x(t)$ is now described as follows:

$$
x(t)=\sum_{n=1}^{N t} c t_{n i}(t) \cdot s_{n i}(t)+c t_{n q}(t) \cdot s_{n q}(t) .
$$




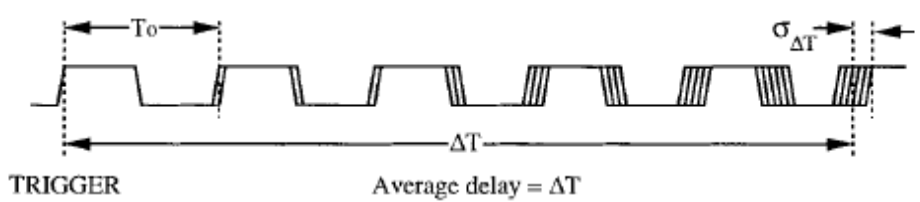

(a)

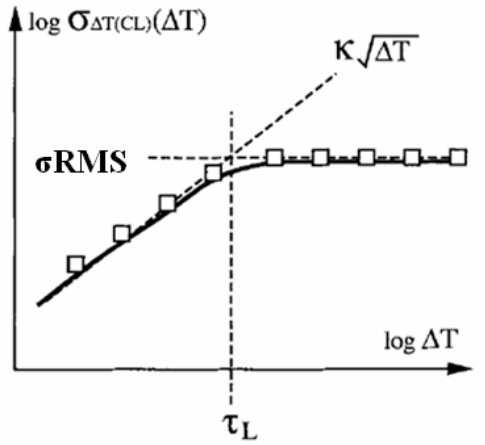

(b)

Fig. 2. Accumulated jitter in PLL [McNeill]. (a) Measurement technique. (b) Jitter versus measurement delay.

\subsection{PLL jitter}

We now determine the characteristics of the jitter that disturbs our analog OFDM system. It is assumed that the jitter coming from the PLL has a Gaussian amplitude distribution with an RMS standard deviation of $\sigma_{R M S}$. The value of this standard deviation is determined by the PLL noise and loop bandwidth. The PLL jitter is modeled as follows. In Fig. 2(a), the waveform at the PLL output is shown versus time. Starting on the left, we see that over time, the phase error accumulates. At delays shorter than the loop bandwidth time constant $\tau_{L}$, the loop is too slow to correct the phase error. From [McNeill], the jitter variance as a function of measurement time in the left area of the plot is:

$$
\sigma_{t}=\kappa \sqrt{\Delta T}
$$

where $\kappa$ is an oscillator (time domain) figure of merit and $\Delta T$ is the accumulation time. Now because the PLL is locked, the phase error is bounded by the loop time constant $\tau_{L}$. In Fig. 2(b), therefore, we can see that the variance of the accumulated phase error first increases versus time, and then becomes limited at $\sigma_{\mathrm{RMS}}$, which is calculated as [McNeill]:

$$
\sigma_{R M S}=\kappa \sqrt{\tau_{L}}
$$

In conclusion, at delays longer than the loop bandwidth time constant, the loop is able to track the reference and the variance is bounded to $\sigma_{\mathrm{RMS}}$. A state-of-the-art jitter figure can be found in the literature: in [vdBeek], a $10 \mathrm{GHz}$ LC-based clock multiplier unit with an RMS jitter $\sigma_{R M S}$ of $0.22 \mathrm{ps}$ at $81 \mathrm{~mW}$ is described. For a ring oscillator, the jitter is somewhat higher: currently around 5ps RMS.

\subsection{Types of inter-carrier interference}

In this section, we outline the different types of inter-carrier interference that are analyzed. The multi-carrier receiver is a 'correlator receiver' (multiplication followed by integrate-anddump) as opposed to the sampling receiver used in most PAM systems. The clock is extracted from the received signal using a clock \& data recovery (CDR) circuit, usually PLL based. The 
locally generated carriers will not be exactly in-phase with the received signal due to PLL jitter. Also, frequency and duty-cycle deviations cause non-orthogonalities. The construction of orthogonal subcarriers in OFDM is quite sensitive to timing non-idealities. In a DMT system, all the timing is carefully observed: the ADC has a low jitter compared to the signal bandwidth and the digital FFT is theoretically perfect. The DMT system makes sure that all these timing non-idealities are absent or at least negligible compared to the channel bandwidth, but in the 'analog OFDM' system this is not always possible.

Because an OFDM system is in its nature quite sensitive to timing non-idealities, we analyze the impact of some of those non-idealities and see how they limit the achievable bit rate in the proposed system. Timing analyses were performed in e.g. [Zogakis], but these are based on a standard DMT system. We analyze our analog OFDM system here. We calculate the effect of jitter and duty-cycle deviations at the receiver side. The following types of inter-carrier interference (ICI) are considered:

(1) crosstalk between in-phase and quadrature signal at a given frequency,

(2) crosstalk between carriers at different frequencies.

This rest of this chapter deals with these different types of inter-carrier interference as follows. First, in section 3.6, we focus on jitter, which causes ICI of type (1). The impact of jitter is analyzed theoretically. Next, in section 3.7, statistical simulations are used to verify those theoretical results, and after that the impact of duty-cycle deviations, causing ICI of both type (1) and (2), is simulated statistically.

\subsection{Impact of jitter on crosstalk between in-phase and quadrature signal at a given frequency}

The goal of this section is to calculate the bit rate limit caused by crosstalk between I\&Q components in a certain subcarrier, caused by jitter in the locally generated carrier. The analysis is based on 'correlation plots', which are introduced in the next sections. This presents an intuitive way of understanding the mechanism of effective amplitude variation of the integrator output caused by jitter. We assume the following:

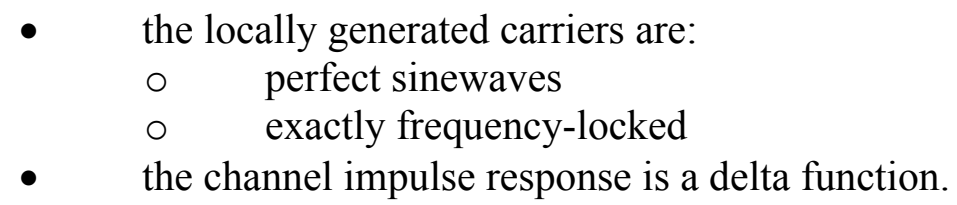

When these assumptions are true, ICI of type (2) is effectively eliminated and we can focus entirely on (1). We also assume that a per-symbol jitter correction (for example based on a pilot-tone) is infeasible because of the high bandwidth.

This section is divided as follows. First, in section 3.6.1, we describe the concept of our jitter analysis. Next, in subsection 3.6.2, the necessary definitions are presented. Following that, we describe and draw the correlation plots (subsection 3.6.3), in order to visualize the I-Q crosstalk. After that, the probability of bit error is calculated (subsection 3.6.4), and finally the calculations of the limits on the achievable bit rate of our system are presented in section 3.6.5. 


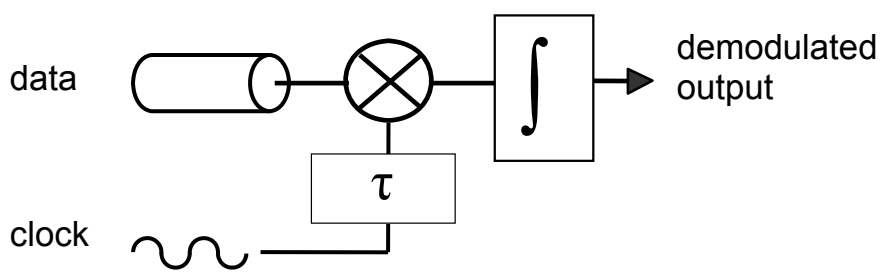

Fig. 3 - Modeling of jitter using delay $\tau$.

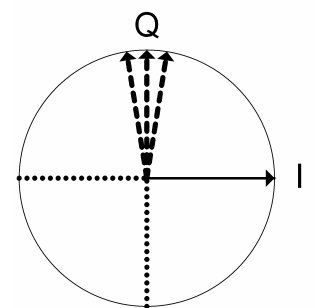

Fig. 4. Jitter in causing I-Q crosstalk. Solid line $=\mathrm{I}$, dashed line $=\mathrm{Q}$.

\subsubsection{Concept of jitter analysis}

Our analysis goal is to determine the change in the integrator output as a function of variations in $\tau$, shown in fig. 3. Ideally, this $\tau$ should be zero, when the clock-and-data recovery (CDR) loop perfectly tracks the transmit clock and provides a carrier with a precisely correct phase. However in practice there is jitter in the receiver generated clock, as described above. We model the jitter in $\tau$, which is thus 'jittering' around its ideal value of zero. This leads to crosstalk between in-phase and quadrature components of a certain subcarrier, and vice versa. This I-Q crosstalk is illustrated in Fig. 4: although we only want to demodulate the transmitted Q component, a small part of the I component will also be present in the demodulated signal.

\subsubsection{Definitions}

The receiver generated in-phase carrier $c_{r, i}(t)$ is defined as:

$$
c_{r, i}(t)=A_{r} \sin \left(2 \pi f_{c} t\right) .
$$

The transmitted, modulated subcarriers $s_{i}(t)$ and $s_{q}(t)$ (resp. in-phase and quadrature component) are defined as:

$$
\begin{aligned}
& s_{i}(t)=A_{i} \sin \left(2 \pi f_{c} t\right), \\
& s_{q}(t)=A_{q} \cos \left(2 \pi f_{c} t\right),
\end{aligned}
$$

where $A_{i}$ and $A_{q}$ are chosen from interval [ $\left.-A_{\max }, A_{\max }\right]$ and $t$ is chosen from the interval $\left(-T_{g t} / 2\right.$, $T_{o p}+T_{g t} / 2$ ) (for one symbol). A guard time (cyclic prefix) $T_{g t}$ is added to the symbol. The following analysis is valid for $-T_{g t} / 2>\tau>T_{g t} / 2$.

\subsubsection{Correlation plots}

The receiver integrates over the time interval $\left(0, T_{o p}\right)$. As a first step, we calculate the (normalized) correlation $z_{i i}(\tau)$ between $s_{i}(t)$ and $c_{r, i}(t)$ : 


$$
z_{i i}(\tau)=\frac{2}{T_{o p}} \int_{0}^{T_{o p}} s_{i}(t-\tau) \cdot c_{r, i}(t) d t=A_{i} A_{r} \cos \left(2 \pi f_{c} \tau\right),
$$

and the correlation $z_{q i}(\tau)$ between $s_{q}(t)$ and $c_{r, i}(t)$ :

$$
z_{q i}(\tau)=\frac{2}{T_{o p}} \int_{0}^{T_{o p}} s_{q}(t-\tau) \cdot c_{r, i}(t) d t=A_{q} A_{r} \sin \left(2 \pi f_{c} \tau\right) .
$$

Mutatis mutandis these calculations (for the in-phase receiver component) deliver the same results for the quadrature receiver component $c_{r, q}(t)$. In fig. 5, example correlation functions are shown $\left(A_{i}=A_{q}=A_{\max }\right)$. The units on the x-axis are $\tau / T_{c}$, where $T_{c}=1 / f_{c}$. The maximum $z_{i i}$ is (by definition) found at $\tau=0$ (optimum match between transmitter and receiver). At $\tau=T_{c} / 4\left(90^{\circ}\right.$ phase shift) $z_{q i}$ is maximum. It can be seen that the time shift between the local carrier and the received signal is very critical for optimum reception.

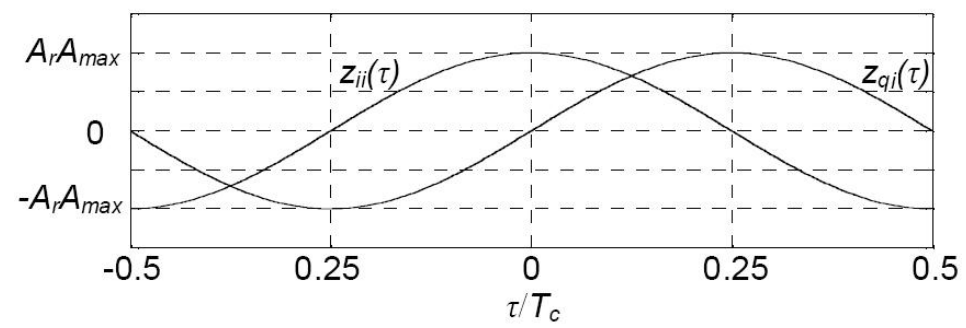

Fig. 5. Correlations $z_{i i}(\tau)$ and $z_{q i}(\tau)$ for $A_{i}=A_{q}=A_{\max }$.

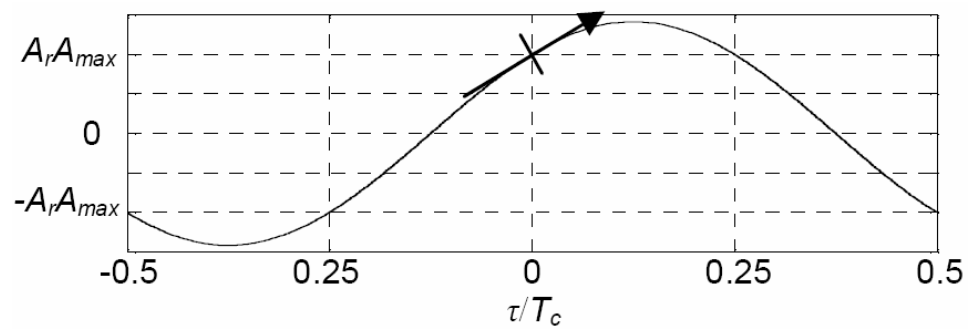

Fig. 6. Correlation $z_{S, A \max , A \max }(\tau)$ with summed signals, and derivative at optimum detection point (arrow) for $A_{i}=A_{q}=A_{\max }$.

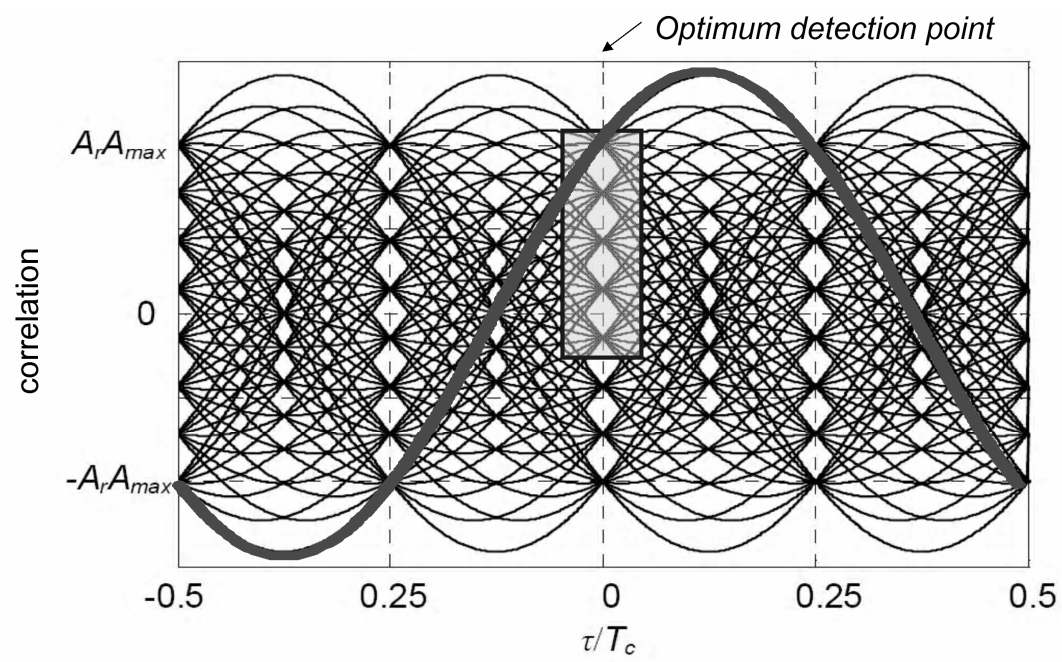

Time difference between local carrier and rcvd signal

Fig. 7. Correlations $z_{s, A i, A q}(\tau)$ for all possible combinations of $A_{i}$ and $A_{q}$. 
Focusing on detection of the in-phase component, we calculate the correlation $z_{s, A i, A q}(\tau)$ of $c_{r, i}(t)$ with the summed transmitted signal, as a function of $A_{i}$ and $A_{q}$,

$$
\begin{aligned}
z_{s, A i, A q}(\tau) & =\frac{2}{T_{o p}} \int_{0}^{T_{o p}}\left(s_{i}(t-\tau)+s_{q}(t-\tau)\right) \cdot c_{r, i}(t) d t \\
= & A_{r}\left(A_{i} \cos \left(2 \pi f_{c} \tau\right)+A_{q} \sin \left(2 \pi f_{c} \tau\right)\right) .
\end{aligned}
$$

This is shown in Fig. 6 (for $A_{i}=A_{q}=A_{\max }$ ) together with the derivative at the optimum detection point. Next, we calculate $z_{s, A i, A q}(\tau)$ for all possible combinations where $-A_{\max }<A_{i}<A_{\max }$ and $-A_{\max }<A_{q}<A_{\max }$. Plotting all these correlations on top of each other looks a bit like a normal eye diagram. In Fig. 7 an example is shown where 3 bits are modulated on both the inphase and quadrature component, resulting in 8 possible levels. The bold line is $z_{s, A \max , \operatorname{Amax}}(\tau)$ (for $A_{i}=A_{q}=A_{\max }$ ) as shown in Fig. 6.

Fig. 7 resembles an eye diagram but it is not the same. Like an eye diagram, these plots can actually be used, in a very similar way, to find the optimum detection moment and to analyze the effect of amplitude and time errors on bit error rate. However, note that the $\mathrm{x}$-axis is not time but relative time shift between $r(t)$ and $c_{r, i}(t)$, in units of $\tau / T_{c}$. The figure shows the effect of a time shift (away from the optimum detection point) on the integrator output.

The impact of a time shift depends on the steepness $y(\tau)$ of the lines around the optimum detection point. We need to calculate this steepness to be able to translate jitter into effective amplitude variation. The steepness is calculated as:

$$
y(\tau)=\frac{d}{d t}\left(z_{s, A i, A q}(\tau)\right)=2 \pi f_{c} A_{r}\left(A_{q} \cos \left(2 \pi f_{c} \tau\right)-A_{i} \sin \left(2 \pi f_{c} \tau\right) .\right.
$$

For $\tau=0, y(\tau)$ is completely determined by $A_{r} A_{q}$, so it can take on $l$ discrete values, where $l$ is the number of levels used in modulation. To be able to translate from time jitter into worst-case amplitude deviation, we calculate the maximum absolute steepness of these lines $y_{\max }$ as

$$
y_{\max }=\max \left(|y(\tau)|_{\tau=0} \mid\right)=2 \pi f_{c} A_{r} A_{\max } .
$$

It is shown in Appendix B that we can safely assume that the jitter accumulation during the integration period is negligible for $\kappa \sqrt{ } T_{o p} \ll T_{c}$, where $T_{o p}$ is the OFDM integration period (equal to the symbol duration minus the guard time), $T_{c}$ the subcarrier period and $\kappa$, as said, is an oscillator figure of merit.

\subsubsection{Probability of bit error}

In this section, a 'tone error rate' $P_{e}$ is calculated. Such a tone error occurs when either the inphase component or the quadrature component of that specific tone (subcarrier) is detected incorrectly. The methodology to estimate the error probability is as follows:

- calculate the effective standard deviation of amplitude of integrator output $\left(\sigma_{A e q}\right)$ as a function of the jitter standard deviation $\left(\sigma_{R M S}\right)$,

- calculate the SNR per tone from $\sigma_{A e q}$ and the distance between levels,

- calculate $P_{e}$ using the cumulative normal distribution function. 
The total system error rate will be limited by the worst performing tone. To avoid having one tone determine the system error rate, the system should be designed to have an equal error rate for each tone.

The receiver compares the integrator output to a number of $(l-1)$ thresholds that are placed in between the amplitude levels. To calculate the error rate, we first need to calculate the probability that the received signal crosses the threshold between two amplitude levels. In Fig. 8 , this is illustrated; $t_{n}$ are the thresholds and $s_{n}$ the signal points. Note that this figure is merely a zoomed-in version of Fig. 7.

The worst-case effective amplitude standard deviation $\sigma_{A e q}$ as a function of the jitter standard deviation is:

$$
\sigma_{A_{e q}}=y_{\max } \sigma_{t}=2 \pi f_{c} A_{r} A_{\max } \sigma_{t} .
$$

We can express the distance between levels $2 d$ as a function of $A_{r} A_{\max }$ as:

$$
2 d=\frac{2 A_{r} A_{\max }}{l-1} .
$$

The error rate is a function of $d / \sigma_{A e q}$, which is equal to the square root of the 'SNR per tone' $S N R_{s m}$. Expressing $d / \sigma_{\text {Aeq }}$ in terms of $f_{c}, \sigma_{t}$ and $l$ gives:

$$
\frac{d}{\sigma_{A_{e q}}}=\sqrt{S N R_{s m}}=\frac{1}{2 \pi \sigma_{t} f_{c}(l-1)} .
$$

To calculate $P_{e}$, we first calculate $P_{i}$, the error rate for the in-phase component, using the Gaussian distribution, and taking into account a factor $(l-1) / l$ because the uppermost and lowermost levels have only one neighbor:

$$
P_{i}=\frac{(l-1)}{l} \frac{1}{\sqrt{2 \pi}} \int_{\frac{d}{\sigma_{A}}}^{\infty} e^{\left(-\frac{y^{2}}{2}\right)} d y=\frac{(l-1)}{l} Q\left(\frac{d}{\sigma_{A_{e q}}}\right),
$$

where $Q(x)$ is the probability that a standard normal random variable with zero mean and unit variance exceeds $x$. Substituting (12) into (13) leads to:

$$
P_{i}=\frac{(l-1)}{l} Q\left(\frac{1}{2 \pi \sigma_{t} f_{c}(l-1)}\right) .
$$

The probability of error $P_{q}$ for the quadrature component is equal to $P_{i}$, because the two components are orthogonal and at the same frequencies, and the noise is Gaussian. The (total) probability of a tone error $P_{e}$ is:

$$
P_{e}=1-\left(1-P_{i}\right)^{2}
$$




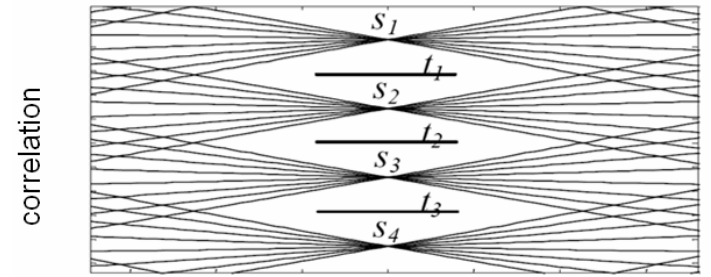

Time difference between local carrier and rcvd signal

(a)

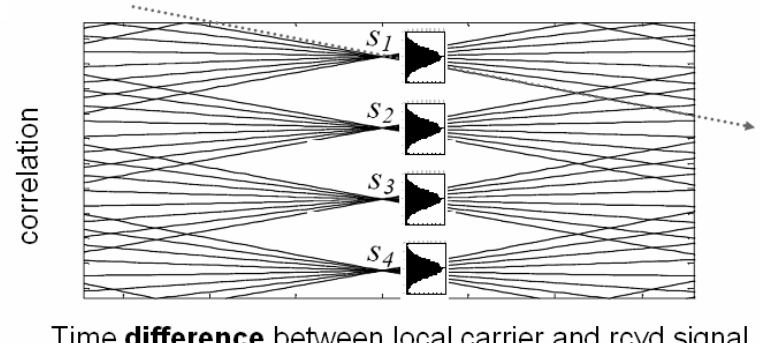

(b)

Fig. 8. Amplitude levels and thresholds. a) Levels and thresholds. b) Translation from timing noise to amplitude noise.

Now we can plot $P_{e}$ (at a given $\sigma_{R M S}$ ) as a function of $f_{c}$ for a number of different modulation depths $n_{b}\left(=\log _{2}(l)\right.$, where $n_{b}$ is expressed in bits). This is shown in Fig. 9 for $\sigma_{R M S}=1$ ps. If necessary, we can convert from tone to bit errors, assuming the use of Gray code, so that one tone error will imply one bit error.

The error rate caused by jitter is a function of modulation depth $n_{b}$ and subcarrier frequency $f_{c}$. The number of bits that can be modulated onto a carrier (for a given error rate) is limited by jitter, with higher frequency carriers being able to carry fewer bits. In an optimum multi-carrier system, the higher frequency carriers should have fewer constellation points to achieve the same error rate. This corresponds with results in [Zogakis].

\subsubsection{Bit rate limits}

To find the bit rate limit of the system, the "max. number of bits that can be modulated" $n_{b, \max }$ was calculated as a function of $\sigma_{R M S}, f_{c}$ and $P_{e}$, using a numeric solver on (18). Fig. 10 shows the outcome for three different values of $\sigma_{R M S}=\{0.1 \mathrm{ps}, 1 \mathrm{ps}, 10 \mathrm{ps}\}$, which corresponds to \{excellent, good, fair $\}$, for $P_{e}=1 \cdot 10^{-12}$.

It is important to know what the jitter limited maximum bit rate of such a multi-carrier system is. This is then compared to a PAM system with an equal bandwidth and error rate. In [Farjad$\mathrm{Rad}]$ a PAM system is described that can achieve a bit rate of $\sim 7 \mathrm{~Gb} / \mathrm{s}$ for an error probability of $\sim 1 \cdot 10^{-12}$, with a bandwidth of $2 \mathrm{GHz}$ and an RMS jitter of $4 \mathrm{ps}$. In our analysis, the upper bound on the multi-carrier system's bit rate is found by integration of $n_{b, \max }$ over a $2 \mathrm{GHz}$ bandwidth and multiplying by two (because both in-phase and quadrature components are used). This delivers a bit rate limit of $14 \mathrm{~Gb} / \mathrm{s}$ (for $\sigma_{R M S}=4 \mathrm{ps}$ and $P_{e}=1 \cdot 10^{-12}$ ). Table 1 summarizes these findings.

The bit rate limit calculated for the multi-carrier system is two times higher than for the PAM system in [Farjad-Rad], but it will have to be corrected downwards for practical implementations. For one thing, the calculation does not include a cyclic prefix. The nonzero duration of the (shortened) impulse response of any practical channel necessitates the use of a guard time. In DMT systems, this guard time is quite a small percentage of the symbol duration because many subcarriers are used and symbols are long. In an analog OFDM system, due to the hardware parallelization with separate mixers, integrate-and-dump blocks and $\mathrm{ADCs} / \mathrm{DACs}$, it is more practical to use only few subcarriers. In that case the cyclic prefix could become long relative to the symbol duration. Furthermore, in an implementation with simple switching mixers, a sine wave on the local oscillator port will generate a square wave on the output. This will produce harmonics that fall onto other tone frequencies, creating unusable areas in the spectrum. 


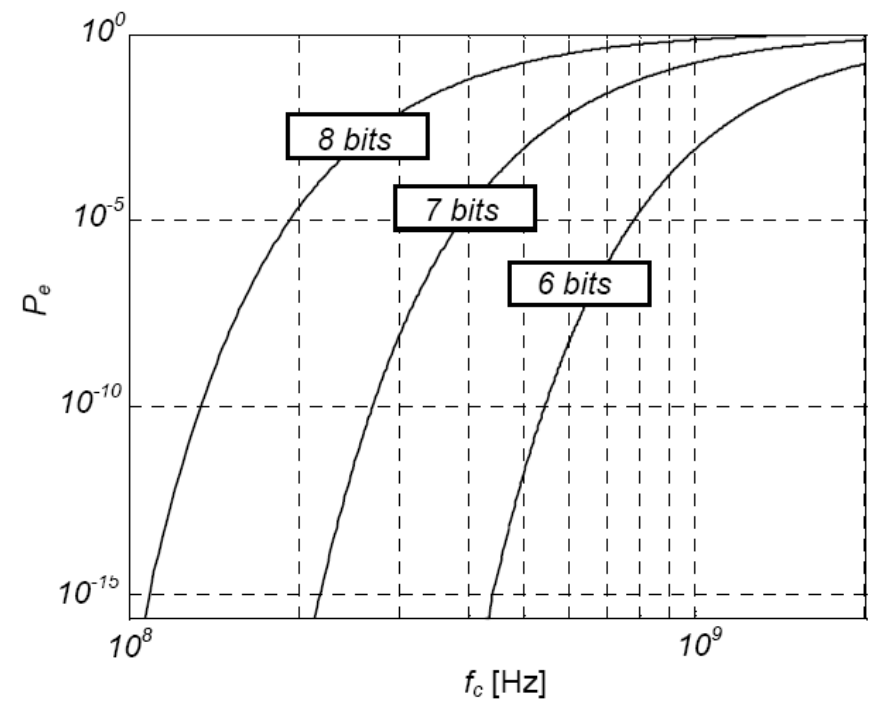

Fig. 9. Probability of error vs. carrier frequency for $\sigma_{R M S}=1 \mathrm{ps}$.

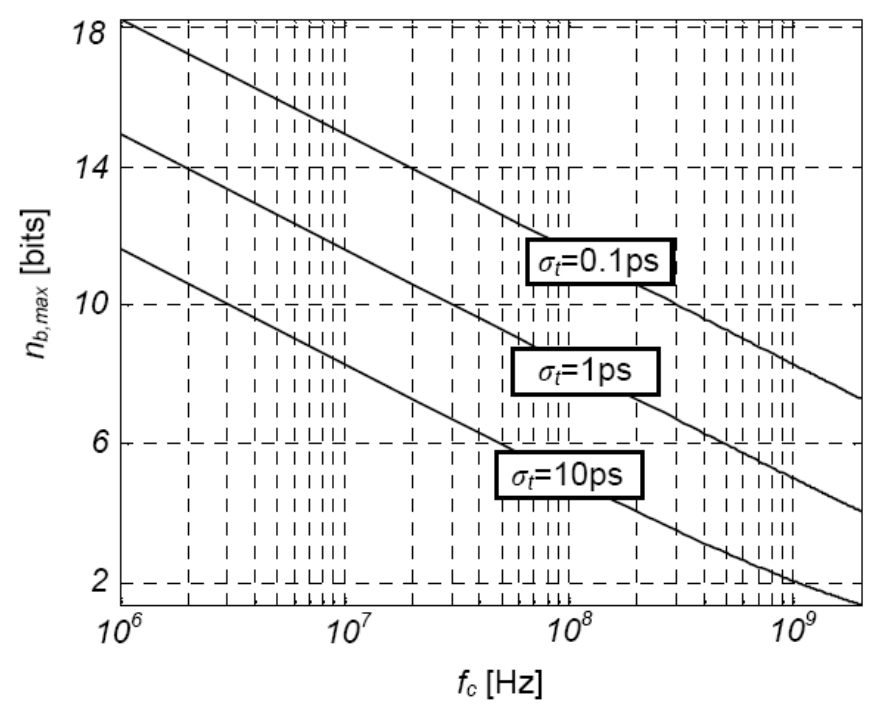

Fig. 10. Maximum number of bits that can be modulated vs. carrier freq. for $P_{e}=1 \cdot 10^{-12}$.

\begin{tabular}{|l|l|l|}
\hline & PAM [Farjad-Rad] & Analog OFDM \\
\hline BER & $10^{-12}$ & $10^{-12}$ \\
\hline Bandwidth & $2 \mathrm{GHz}$ & $2 \mathrm{GHz}$ \\
\hline RMS jitter & $4 \mathrm{ps}$ & $4 \mathrm{ps}$ \\
\hline Capacity & $7 \mathrm{~Gb} / \mathrm{s}$ (meas.) & $14 \mathrm{~Gb} / \mathrm{s}$ (theory) \\
\hline
\end{tabular}

Table 1. Comparison between measured PAM system and proposed Analog OFDM system.

\subsection{Statistical jitter simulations}

In this section, we present statistical simulations regarding ICI of both type (1) and (2). First, subsection 3.7.1, we describe the use of statistical simulations to check the previous analytical calculations which estimated the influence of jitter on the error rate of the system (ICI of type (1)). Next, in subsection 3.7.2, the impact of duty-cycle deviations is analyzed, which causes both ICI type (1) and ICI type (2). 


\subsubsection{ICI type (1) as a consequence of jitter}

We calculate an effective SNR as a function of jitter, and from this SNR calculate the error rate. It is again assumed that the jitter coming from the PLL has a Gaussian time distribution with an RMS variance of $\sigma_{R M S}$. Its size is determined by the PLL noise and loop bandwidth. From the calculations in the previous section, the effective SNR as a function of this jitter is expected to be dependent on carrier frequency $f_{c}$ and number of levels $N_{l}$ used in modulation. In the simulations, an OFDM symbol is used which is filled with only two subcarriers at one frequency $f_{c}$ : the in-phase and the quadrature component. The length of the orthogonality period $T_{o p}$ is $1 / f_{c}$. The received waveform is then multiplied with a randomly circularly shifted subcarrier. The circular shift is a white Gaussian random variable. The channel impulse response is a delta function, so the cyclic prefix length has no impact on the results. (Furthermore, the modeled receiver jitter only affects the carrier phase and does not modulate the symbol edges, which would have an impact for zero cyclic prefix length.)

We run the following simulations:

- $f_{c}=0.5 \mathrm{GHz}, N_{l}=4, \sigma_{R M S}=10 \mathrm{ps}$,

- $f_{c}=2.5 \mathrm{GHz}, N_{l}=4, \sigma_{R M S}=10 \mathrm{ps}$,

- $f_{c}=0.5 \mathrm{GHz}, N_{l}=16, \sigma_{R M S}=10 \mathrm{ps}$.

A jitter of 10ps is assumed because this enables us to detect errors over a short simulation (time step size $=1 \mathrm{ps}$ ). Statistical simulations are made with 500 symbols. We focus on the error rate for the in-phase component. We analyze crosstalk between in-phase and quadrature component of a single carrier as a function of variance in $\tau$ caused by jitter. The jitter was modeled by adding a white Gaussian random variable to the receiver time axis. The SNR per subcarrier values were calculated by sampling the data, demodulating the data and fitting it to a normal distribution. The fitting routine estimates the amplitude variance $\sigma_{A}$ of the integrator output which is used to obtain the SNR per subcarrier $S N R_{s m}$ as (see also previous section):

$$
S N R_{s m}=\left(\frac{d}{\sigma_{A}}\right)^{2}
$$

where $2 d$ is the distance between two adjacent levels. Next, the probability of error $P_{e}$ for the in-phase component can be calculated as:

$$
P_{e}=Q\left(\sqrt{S N R_{s m}}\right)
$$

We use histograms of the integrator output to graphically show the variance $\sigma_{A}$. When we add a jitter of $\sigma_{R M S}=10 \mathrm{ps}$ in the receiver generated carrier, and the frequency and number of levels are set at $f_{c}=0.5 \mathrm{GHz}$ and $N_{l}=4$ levels ( $=2$ bits) we can transmit data at a low BER. This is shown in Fig. 11. From the simulation, the calculated $S N R_{s m}=25 \mathrm{~dB}$; this gives an excellent $P_{e} \ll 1 \cdot 10^{-12}$. Using the formula derived in the previous section, we obtain $S N R_{S m}=20.5 \mathrm{~dB}$. Analytical and statistical results correspond to within $5 \mathrm{~dB}$. This difference can be explained from the fact that the analysis takes the worst case steepness as per Eq. (13), and therefore arrives at a more pessimistic SNR estimation than the simulation. (The actual steepness is a function of the levels of the I and Q signals.) 


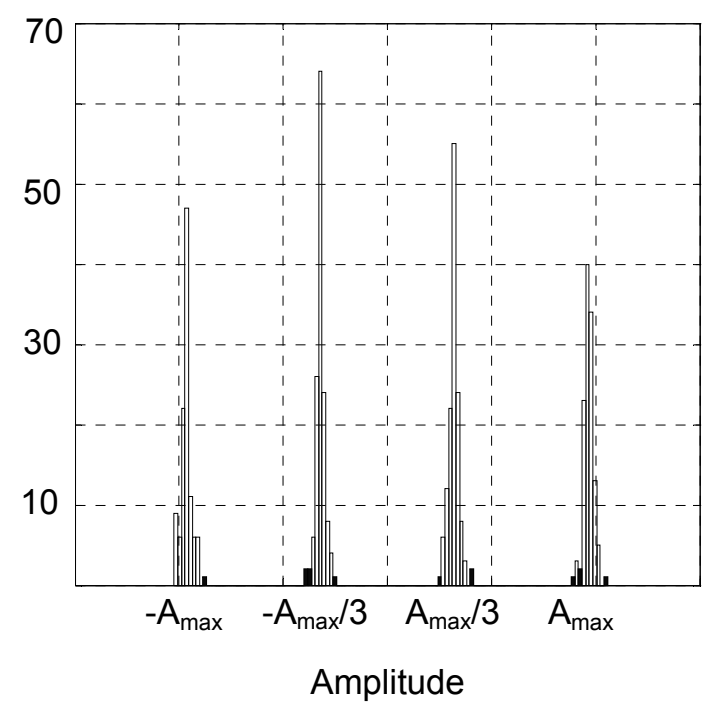

Fig. 11. Output hist. (jitter $\sigma_{R M S}=10 \mathrm{ps}, f_{c}=0.5 \mathrm{GHz}, N_{l}=4$ ).

However, when we try to increase the frequency to $f_{c}=2.5 \mathrm{GHz}$ (number of levels remains $N_{l}=4$ ), the jitter starts to have a severe impact, causing $S N R_{s m}=10 \mathrm{~dB}$, which gives a poor $\mathrm{Pe}=1 \cdot 10^{-3}$. This is shown in Fig. 12. Calculated analytically, we obtain an $S N R_{s m}=6.5 \mathrm{~dB}$.

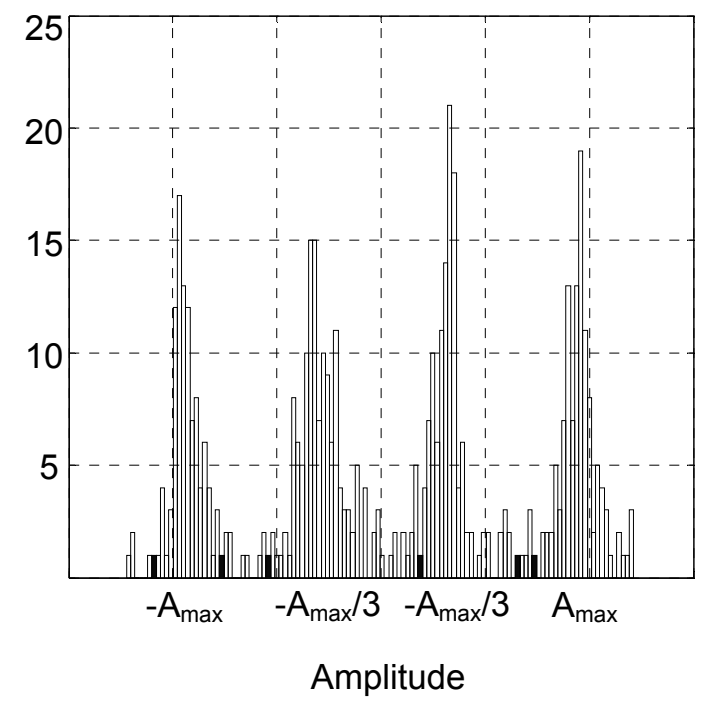

Fig. 12. Output hist. (jitter $\sigma_{R M S}=10 \mathrm{ps}, f_{c}=2.5 \mathrm{GHz}, N_{l}=4$ ).

A comparable jitter impact can be seen in Fig. 13 when we do not increase $f_{c}$ (it remains at $\left.f_{c}=0.5 \mathrm{GHz}\right)$ but instead increase the modulation depth to $N_{l}=16$ levels. The signal-to-noise ratio per symbol $S N R_{S m}=12 \mathrm{~dB}$, which gives an equally poor $\mathrm{Pe}=1 \cdot 10^{-6}$. Using the formula derived in the previous section, we obtain an $S N R_{S m}=6.5 \mathrm{~dB}$.

In conclusion, we observe that, for a given jitter RMS variance, low frequency tones can carry more bits than higher frequency tones for the same error rate. This confirms the previous analysis. 


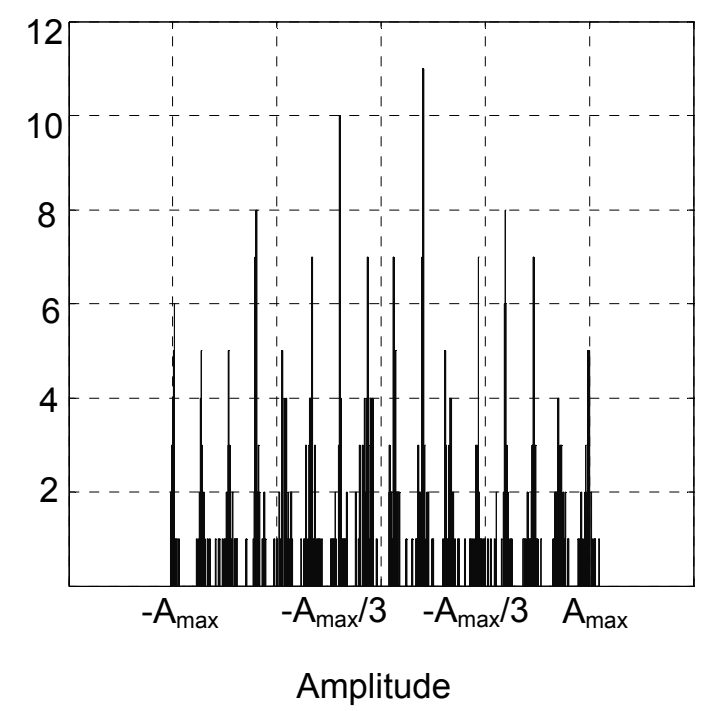

Fig. 13. Output hist. (jitter $\sigma_{R M S}=10 \mathrm{ps}, f_{c}=0.5 \mathrm{GHz}, N_{l}=16$ ).

A per-symbol jitter correction could be applied as in DMT systems, but the question is whether the electronics are fast enough to allow this. Leaving out the quadrature component would solve the jitter problems analyzed above, but at the cost of half the capacity. That would undo all of our gain in bit rate over the PAM system.

\subsubsection{Impact of duty-cycle variations on carrier orthogonality}

In this subsection we look at deterministic deviations and/or variations in the duty cycle. They can cause both type (1) and type (2) ICI. We simulate these effects for a system with two tone frequencies $\left(f_{2}=2 f_{1}\right)$ using square-wave carriers, where each frequency is modulated with both in-phase and quadrature components. (Therefore the total number of carriers is four.) The dutycycle $d$ of the receiver generated in-phase carrier $c r_{l, i}$ at $f_{l}$ is varied, while the duty-cycle of all the transmitted carriers is exactly $50 \%$. Below, a short summary of the simulation setup is given.

Transmitter carriers:

- $\quad c t_{1, i}: f_{c}=f_{l}$, in-phase component

- $\quad c t_{1, q}: f_{c}=f_{l}$, quadrature component

- $\quad c t_{2, i}: f_{c}=f_{2}$, in-phase component

- $\quad c t_{2, q}: f_{c}=f_{2}$, quadrature component.

Receiver carrier:

- $\quad c r_{1, i}: f_{c}=f_{l}$, in-phase component, deviations in duty-cycle.

We calculate the following correlations as a function of the duty-cycle of $c r_{1, i}$ :

- $\quad \operatorname{corr}_{1}=$ between $c r_{l, i}$ and $c t_{l, i}$

- $\quad \operatorname{corr}_{2}=$ between $c r_{1, i}$ and $c t_{1, q}$

- $\quad \operatorname{corr}_{3}=$ between $c r_{1, i}$ and $c t_{2, I}$

- $\operatorname{corr}_{4}=$ between $c r_{1, i}$ and $c t_{2, q}$.

These are all the possible correlations for this two-tone system. 


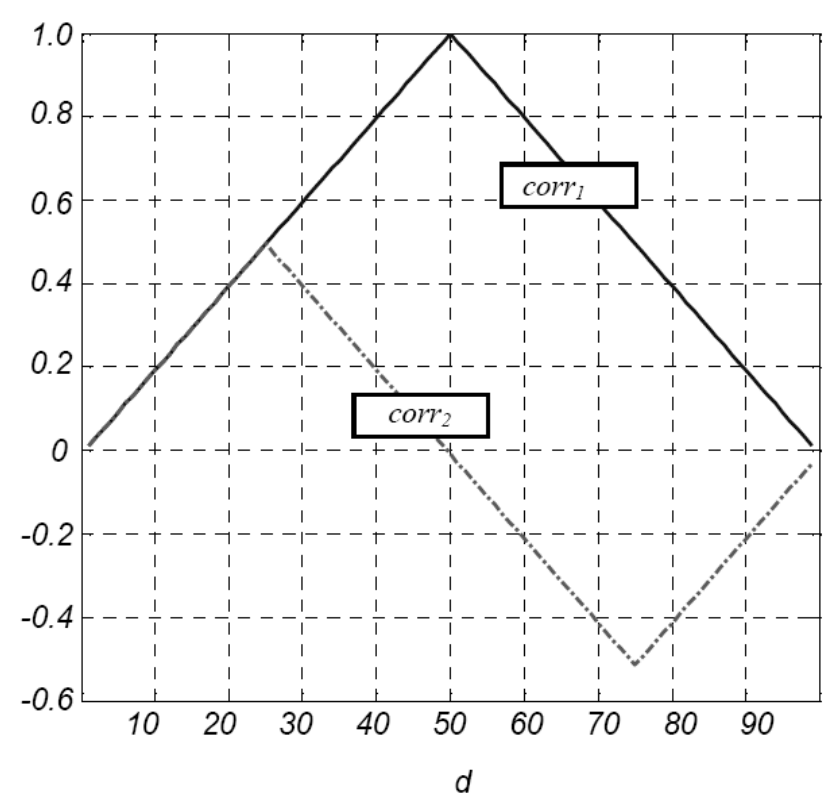

Fig. 14. $\operatorname{corr}_{1}$ and $\operatorname{corr}_{2}$ versus duty cycle $d$.

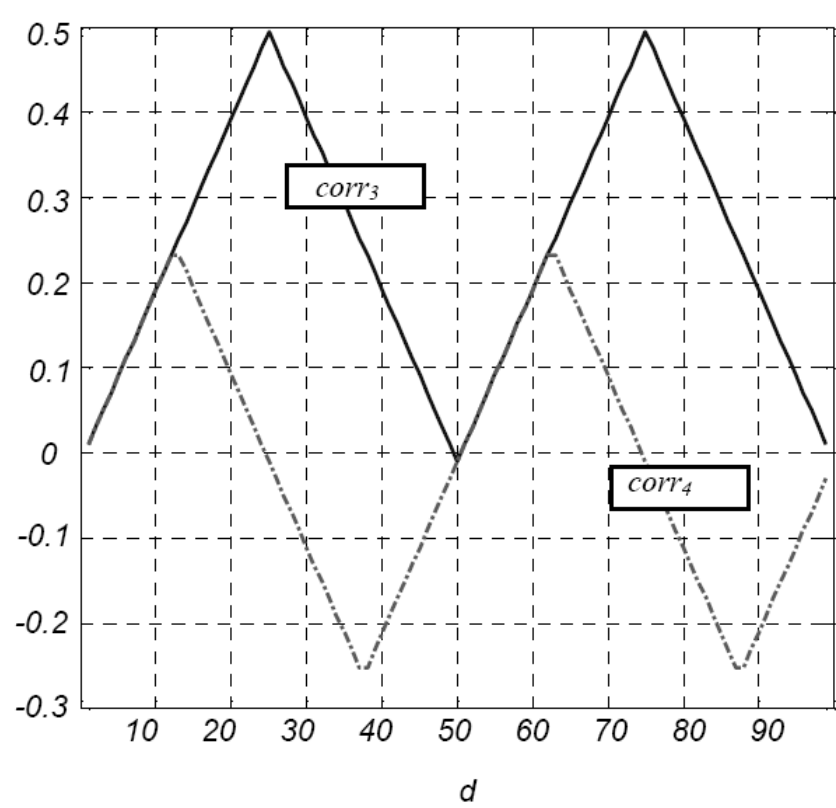

Fig. 15. $\operatorname{corr}_{3}$ and corr $_{4}$ versus duty cycle $d$.

Fig. 14 shows the correlation of $c r_{1, i}$ with the desired transmitted signal $c t_{1, i}$ and with the quadrature component $c t_{l, q}$ at that same frequency (respectively $\operatorname{corr}_{1}$ and $\operatorname{corr}_{2}$ ). Fig. 15 shows the correlation of $c r_{1, i}$ with the double frequency carriers $c t_{2, i}$ and $c t_{2, q}\left(\operatorname{corr}_{3}\right.$ and $\operatorname{corr}_{4}$ respectively). The values were normalized to one.

All deviations from the 50\% duty-cycle introduce inter-carrier interference (crosstalk) and lead to a limited SNR, as shown in Fig. 16. The peak at 50\% corresponds to an infinite SNR.

The error rate as a function of the duty cycle is shown in Fig. 17. The observation to be made is that even this simple multi-carrier system is very sensitive to duty-cycle variations, with the error rate $P_{e}>1 \cdot 10^{-6}$ for $>5 \%$ variation. Also, in case of frequency mismatch or duty-cycle mismatch, inter-carrier interference with carriers at other frequencies will arise. 


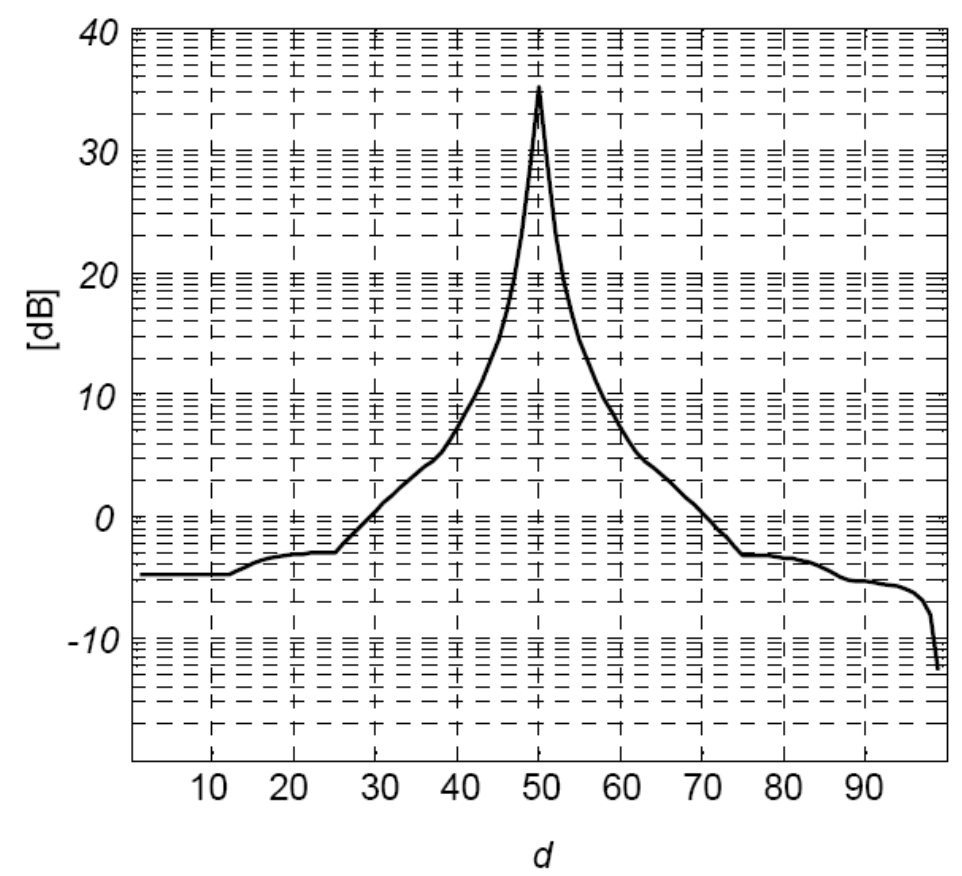

Fig. 16. SNR as a function of duty cycle $d$.

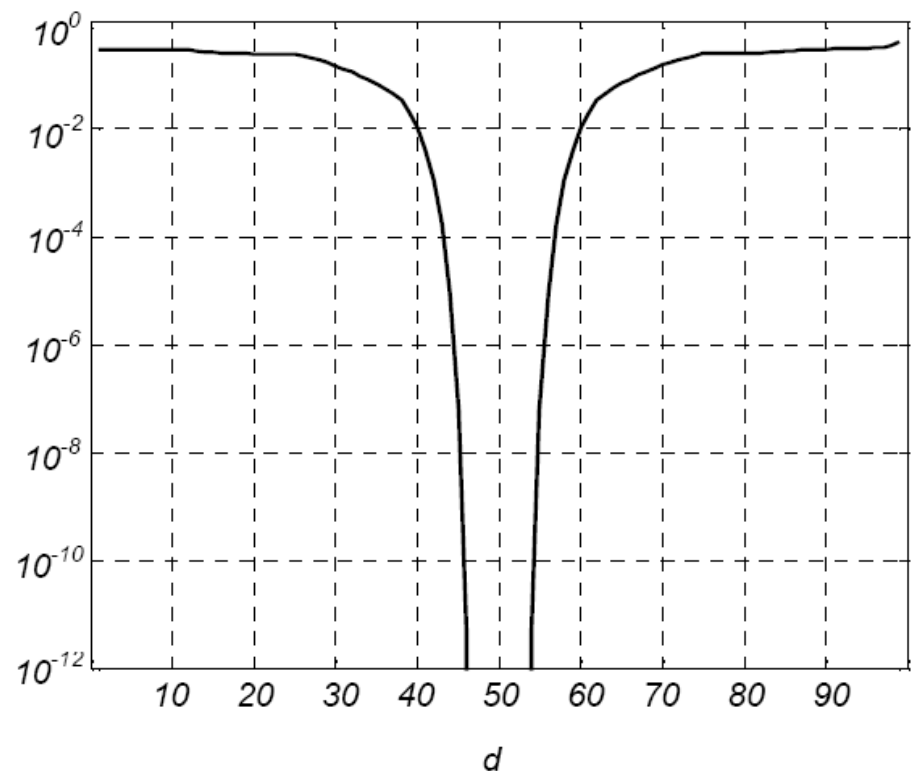

Fig. 17. Error rate $P_{e}$ as a function of duty cycle $d$.

\subsection{Related work from the literature}

In [Amirkhany-1], work parallel to that presented above was carried out in the form of an analysis of an analog multitone (AMT) system. A system with 15 ADCs is proposed, which is analyzed using a convex optimization framework, with linear transfer matrices to model the inter-carrier interference (ICI). The outcome of the analysis is that an improvement of approximately a factor two in bit rate can be achieved over a PAM serial link, provided that "RF circuits with the characteristics specified ... could be built in CMOS technology". As a follow-up on this work, in [Amirkhany-2], a transmitter was presented, but the receiver was not built. Instead, a very high-speed high-resolution ADC was used to sample the signal and all the data demodulation and equalization was done off-line. It remains to be seen whether such a receiver is feasible, especially in terms of jitter requirements. It is also concluded that 
multitone techniques are most advantageous on channels with spectral notches, and that ICI cancellation is one of the biggest challenges in building such analog multitone systems.

\subsection{Conclusions}

The feasibility of an 'analog OFDM' system is analyzed. A transceiver architecture for gigabit multi-carrier transmission over copper channels is discussed. Multiple parallel DACs and ADCs are used. Harmonics caused by switching mixers hinder the use of a large number of carriers. We have examined the impact of several timing non-idealities on system SNR, BER and capacity. Jitter, coming from the PLL, causes crosstalk between the in-phase and quadrature components. This limits the maximum bit rate which can be achieved, given a certain specification for the symbol error rate. The jitter is assumed to have a Gaussian amplitude distribution. It is concluded that low frequency tones can carry more bits than higher frequency tones for the same error rate. Using correlation plots, this can be understood. A jitter limit on the system bit rate is calculated by integrating the area under the plot of "maximum number of bits that can be modulated" versus carrier frequency. These analytical results were confirmed by running statistical simulations. Furthermore, duty-cycle deviations even cause crosstalk between carriers at different frequencies. A duty cycle deviation of more than 5\% already causes the error rate of a simple two frequency multi-carrier system to drop below $10^{-6}$.

It seems that traditional Pulse Amplitude Modulation (PAM) systems with a comparable bandwidth still are the better choice for channels with a loss that increases monotonously with frequency. OFDM techniques might still be attractive when the channel suffers from severe reflections, such as in some PCB tracks (and of course when it suffers from ingress noise as in ADSL systems). 


\section{Chapter 4}

\section{PWM pre-emphasis}

\subsection{Introduction}

To compensate for channel losses, transmitter pre-emphasis or receiver equalization can be applied [Farjad-Rad], [Lee], [Kudoh], [Gai], [Dally-1]. Receiver equalization typically involves several analog blocks which impose speed, accuracy and noise requirements. On the other hand, transmitter pre-emphasis allows the use of a simple receiver that only needs to sample binary values [Dally-1]. Pre-emphasis methods found in the literature are commonly based on symbol-spaced Finite Impulse Response (SSF) filtering [Farjad-Rad], [Lee], [Kudoh], [Gai], [Dally-1].

In this chapter, we describe the application of PWM pre-emphasis to the equalization of copper cables and PCBs. It provides an alternative to FIR pre-emphasis with advantages in the light of developments in CMOS scaling. In our group, PWM pre-emphasis was successfully applied to the equalization of on-chip wires [Schinkel-1], [Schinkel-2]. In this chapter, it is shown that PWM pre-emphasis provides a higher maximum loss compensation (30dB) than the commonly used 2-tap SSF filter (20dB), because its transfer function happens to fit very well to the copper channel. Only one parameter needs to be adjusted to the channel (the duty-cycle), unlike with 5-tap SSF filters, or multi-stage receiver equalizers, which can also achieve $30 \mathrm{~dB}$ loss compensation but with higher complexity. This chapter is based on our existing publications [Schrader-4], [Schrader-5], [Schrader-6] and [Schrader-7].

Section 4.2 of this chapter explains the principle behind PWM pre-emphasis, and section 4.3 analytically compares FIR and PWM pre-emphasis in the frequency domain. Section 4.4 shows time domain simulations. In section 4.5, the design and test results of three chips are discussed. Measurements are given for cables and for PCBs. Finally, conclusions are drawn in section 4.6.

\subsection{Pulse-width modulation pre-emphasis}

In Fig. 1(a), the output voltage waveform for the PWM-PE filter is shown. The output is normalized to +/- 1V. We assume that the modulation scheme is 2-level Pulse Amplitude Modulation (PAM). The PWM pulse shape resembles a Manchester-coded signal, but whereas the Manchester duty-cycle is fixed at 50\%, the PWM signal instead has a tunable duty-cycle. A duty-cycle of $100 \%$ corresponds to transmission of a normal polar NRZ data signal without pre-emphasis, and 50\% to transmission of a Manchester-coded data signal (maximum pre-emphasis setting). The optimum duty-cycle is somewhere in between, depending on the channel characteristics. In comparison, Fig. 1(b) shows the output of a 2-tap symbol-spaced FIR (SSF) transmitter, again normalized to +/- 1V. Both types of PE need only one adjustable parameter to fit them to the channel, making a coefficient-finding algorithm converge in a quick and straightforward manner. (For the 2-tap SSF this parameter is the ratio between the two tap weights.) 


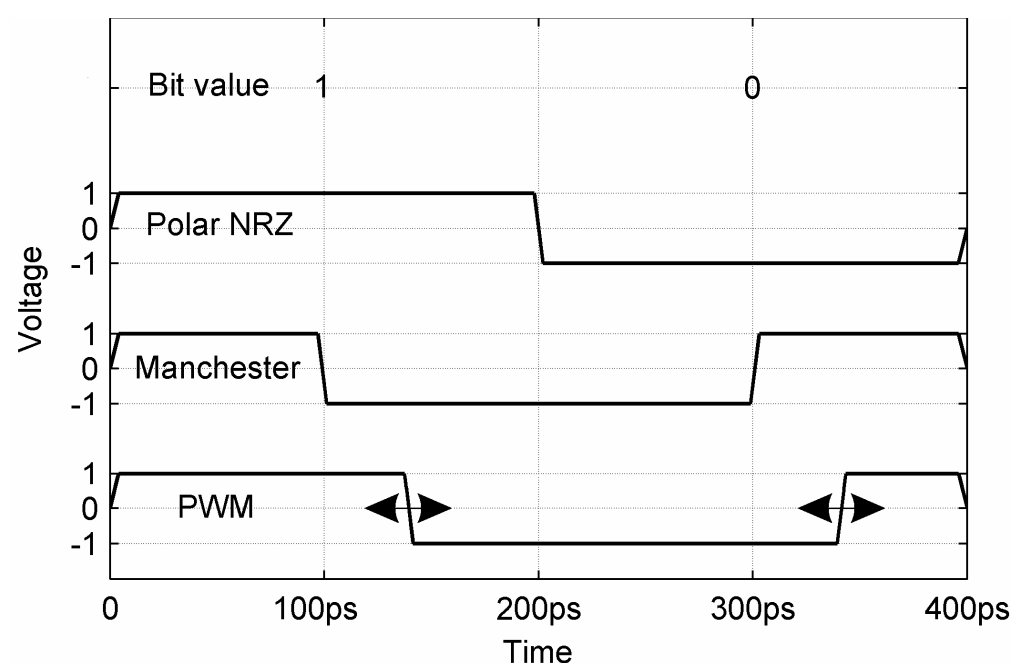

(a)

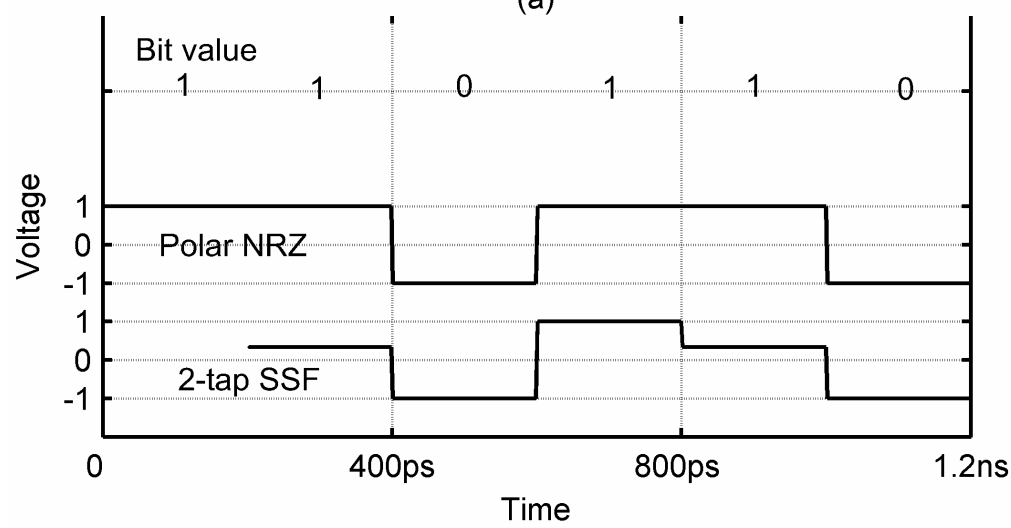

(b)

Fig. 1. Output signals for PE transmitters, $T_{s}=200$ ps. ( $T_{s}$ is defined as the symbol length.)

(a). PWM pre-emphasis. (b). 2-tap SSF pre-emphasis.

For a quick insight into PWM-PE filtering, the simulated time domain response of a $25 \mathrm{~m}$ lowcost, low-end, standard RG-58CU cable to PWM pulses with several duty-cycles and $T_{s}=200 \mathrm{ps}$ is shown in Fig. 2. This cable is used later in the experiments. The time domain cable model is explained in Chapter 2 and includes both skin-effect and dielectric loss. The sample moment $t_{s}$ is shown with a triangle, and the ISI contributions are shown with circles. Note that for the duty-cycle setting of $53 \%$, the cable output pulse becomes much narrower than the response to a plain polar NRZ pulse (100\%). This reduces the ISI contributions significantly. It is seen that an optimum setting can be found at which the ISI is minimized. Second, note that the optimum duty-cycle for this particular channel is near - but not equal to $-50 \%$. As a comparison, in Fig. 3 the response of the channel to 2-tap SSF pulses is shown. (Parameter $r$ is described below.) It can be seen that PWM-PE is capable of narrowing the channel pulse response, similar to FIR pre-emphasis.

In practice, PWM duty-cycle $d$ can be adapted to the channel automatically using return channel communication and a control algorithm. The need for a return channel is a disadvantage compared to receiver equalization, but it is common among all pre-emphasis approaches. A sign-sign block least mean squares (LMS) algorithm can be used as shown in [Stonick]. Such a control algorithm could also compensate for temperature and channel variations. Convergence of the LMS algorithm for the single-coefficient PWM-PE filter is more straightforward than it would be for a filter with multiple coefficients. 

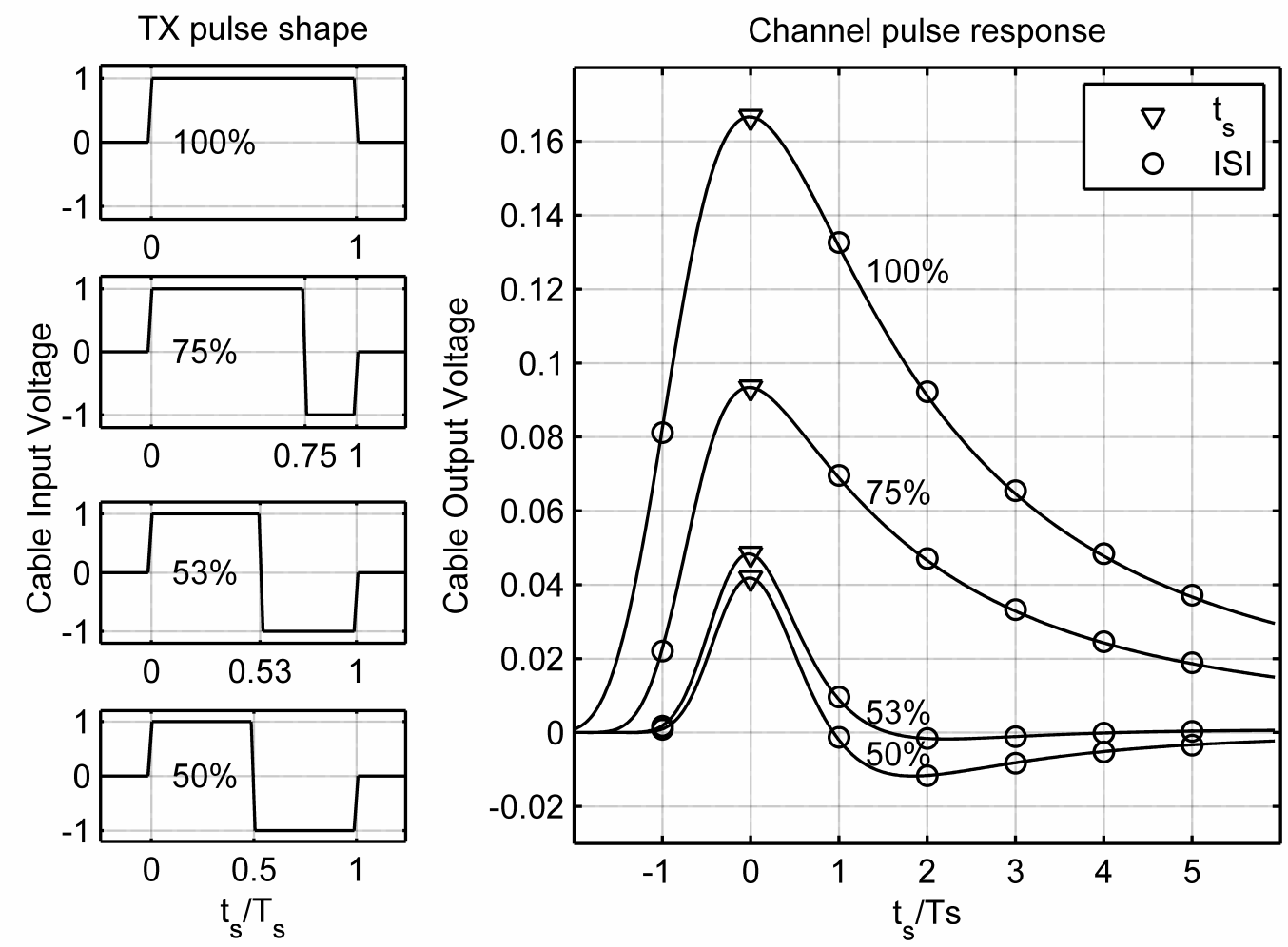

Fig. 2. TX pulse shapes (Ts=200ps) of PWM-PE filter with varying duty-cycles and simulated responses of $25 \mathrm{~m} \mathrm{RG}-58 \mathrm{CU}$ cable.
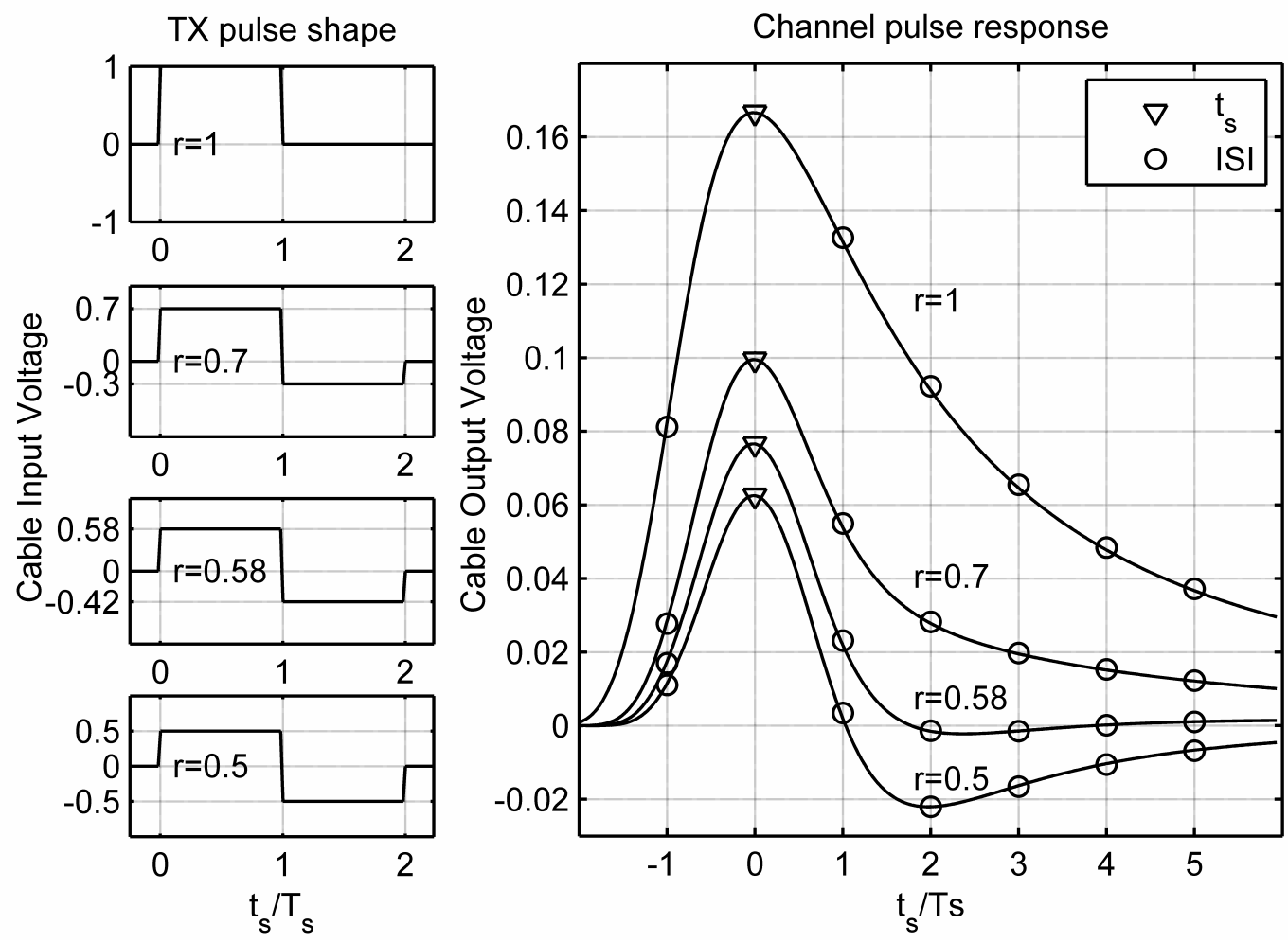

Fig. 3. TX pulse shapes (Ts $=200 \mathrm{ps})$ of SSF-PE filter with varying $r$ parameter and simulated responses of $25 \mathrm{~m} \mathrm{RG}-58 \mathrm{CU}$ cable. 
By replacing FIR pre-emphasis with PWM pre-emphasis (PE), amplitude resolution requirements are replaced with timing resolution requirements, which is beneficial in the light of technology scaling. Future CMOS technologies will have lower voltage headroom and offer higher switching frequencies. PWM-PE exploits the timing resolution available in modern CMOS processes.

\subsection{Frequency domain comparison}

To be able to calculate the frequency transfer functions of the filters and compare them, the well-known stochastic method for calculation of the Power Spectral Density (PSD) is used as a basis [Couch]. The stochastic approach gives the PSD for a random data sequence. This method is described briefly below. We first calculate the PSD and transfer function of PWM pre-emphasis (subsection 4.3.1), then calculate the PSD and transfer function of the 2-tap SSF filter (subsection 4.3.2). These transfer functions are compared in 4.3.3. Next, in 4.3.4 we compare the flatness of the equalized channel transfer functions. Finally, in subsection 4.3.5 we comment on the high-frequency (HF) behavior of the PWM per-emphasis filter.

\subsubsection{PSD and transfer function of PWM pre-emphasis}

First, the transmitted data pattern $\operatorname{data}_{t r}(t)$ is defined as:

$$
\operatorname{data}_{t r}(t)=\sum_{n=-\infty}^{\infty} a_{n} p\left(t-n T_{s}\right)
$$

where $a_{n} \in\{-1,1\}$ denotes the random data, $T_{s}$ is the symbol duration, and $p(t)$ is the pulse shape. Both levels -1 and 1 for $a_{n}$ are equally likely to occur. For example, $a=\{. ., 1,-1,-1,1,-1,1,1, \ldots\}$. The PWM pulse $p(t)=p_{p w m}(t)$ is defined as follows (illustration in Fig. 4(a)):

$$
p_{p w m}(t)=\left\{\begin{array}{cc}
0, & t<-T_{s} / 2, \\
1, & -T_{s} / 2 \leq t<(d-1 / 2) T_{s}, \\
-1, & (d-1 / 2) T_{s} \leq t<T_{s} / 2, \\
0, & T_{s} / 2 \leq t,
\end{array}\right.
$$

where $d$ denotes the duty-cycle $\left(0.5<d<1\right.$ fits best to copper cables) and $T_{s}$ denotes the symbol duration.

The PSD PSD $(\omega)$ of the stochastic signal can now be calculated using the formula [Couch]:

$$
P S D(\omega)=\frac{|P(\omega)|^{2}}{T_{s}} \sum_{k=-\infty}^{k=\infty} R(k) e^{j k \omega T_{s}}
$$

where $P(\omega)$ is the Fourier transform of $p(t)$, or in other words the pulse spectrum, and $R(k)$ is the autocorrelation of the random data sequence $a_{n}$. 
The autocorrelation $R(k)$ is the same as for polar NRZ signaling, and is calculated as follows [Couch]:

$$
R(k)=\sum_{i=1}^{I}\left(a_{n} a_{n+k}\right)_{i} P_{i}=\left\{\begin{array}{cc}
A^{2}, & k=0, \\
0, & k \neq 0,
\end{array}\right.
$$

where $a_{n}$ and $a_{n+k}$ are the multiplication factors for the data pulses at the $n$th and $n+k$ th symbol positions, respectively, and $P_{i}$ is the probability of having the $i$ th $a_{n} a_{n+k}$ product. We use polar signaling $\left(a_{n}\right.$ can be either $-A$ or $A$ ). $A=1$ in our case. $I$ is the number of possibilities for the product values, which is equal to 4 for polar NRZ (and the PWM pre-emphasis filter).

We can now calculate the PSD. The TX output swing is normalized to $+/-1 \mathrm{~V}$ (as illustrated in Fig. 2). The spectrum $P_{p w m}(f)$ of the PWM pulse is calculated by taking the Fourier transform of $p_{p w m}(t)$ :

$$
P_{p w m}(f)=\int_{-\infty}^{\infty} p_{p w m}(t) e^{-j \omega t} d t=\int_{-T_{s} / 2}^{(d-0.5) \cdot T_{s}} e^{-j \omega t} d t+\int_{(d-0.5) \cdot T_{s}}^{T_{s} / 2}-e^{-j \omega t} d t
$$

Simplifying leads to:

$$
P_{p w m}(f)=\frac{2}{j \omega}\left(\cos \left(\omega T_{s} / 2\right)-e^{-j \omega(d-0.5) T_{s}}\right) .
$$

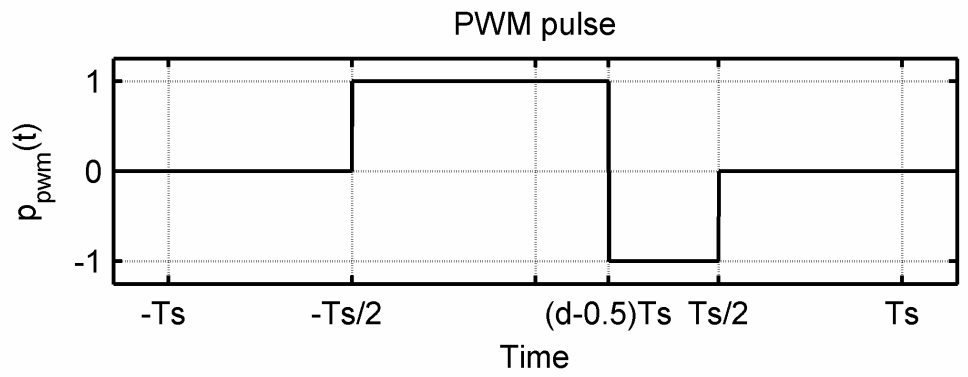

(a)

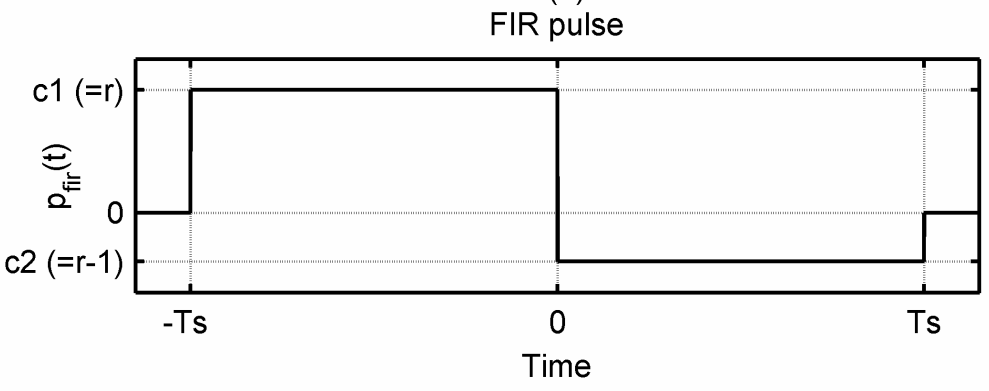

(b)

Fig. 4. Definition of pulse shapes. (a) PWM-PE. (b) 2-tap SSF-PE. 


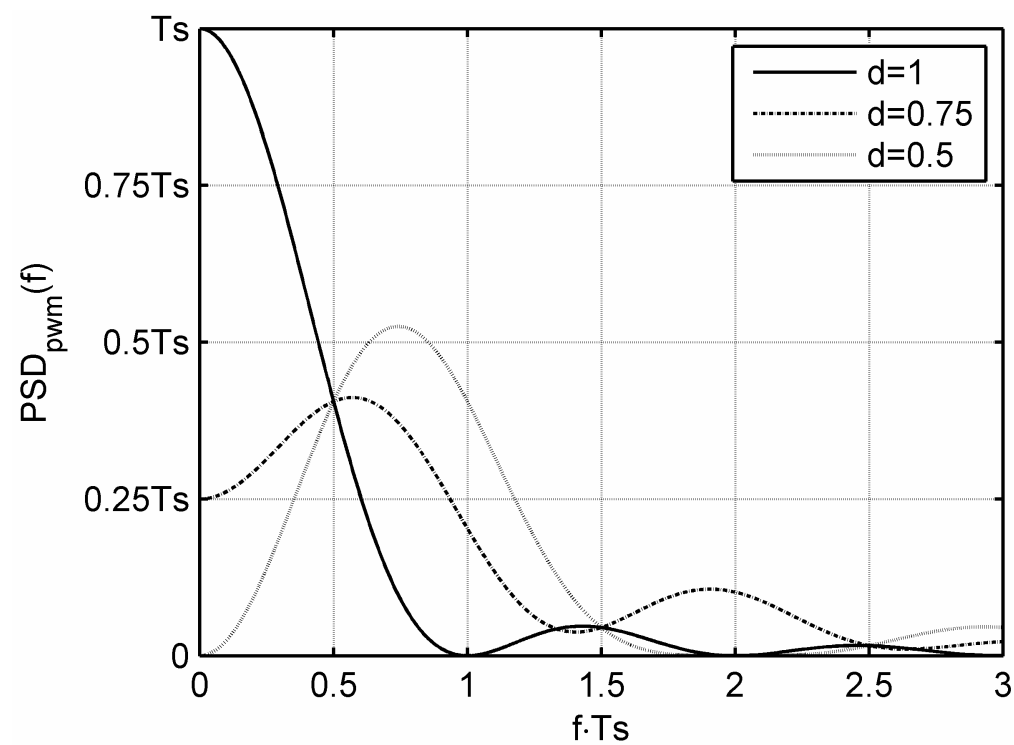

Fig. 5. Power spectral densities for the PWM signal.

Now we calculate the power spectral density $P S D_{p w m}$ for the PWM filter:

$$
\begin{aligned}
& P S D_{p w m}(\omega)=\frac{\left|P_{p w m}(\omega)\right|^{2}}{T_{s}} \sum_{k=-\infty}^{k=\infty} R(k) e^{j \omega k T_{s}} \\
& =2 \frac{\cos \left(\omega T_{s}\right)-2 \cos \left(\omega d T_{s}\right)-2 \cos \left(\omega(d-1) T_{s}\right)+3}{\omega^{2} T_{s}} .
\end{aligned}
$$

This function is shown in Fig. 5. The situation with $d=1$ corresponds to normal polar NRZ line coding, and the situation with $d=0.5$ corresponds to Manchester line coding. Note how the spectrum is shaped and moved to higher frequencies by the (time-varying) PWM filter.

The frequency domain transfer function $H_{p w m}(f)$ of the PWM-PE filter can now be calculated as follows (because $R(k)$ is the same for PWM as for polar NRZ):

$$
H_{p w m}(f)=\frac{P_{p w m}(f)}{P_{N R Z}(f)},
$$

where $P_{N R Z}(f)$ is the spectrum of a normal polar NRZ pulse of width $T_{s}$ and height 1 , which is well known to be:

$$
P_{N R Z}(f)=\frac{2}{\omega} \sin \left(\omega T_{s} / 2\right)
$$

The expression for $H_{p w m}(f)$ now becomes:

$$
H_{p w m}(f)=\frac{\cos \left(\omega T_{s} / 2\right)-e^{-j \omega(d-0.5) T_{s}}}{j \sin \left(\omega T_{s} / 2\right)} .
$$


Taking the modulus yields:

$$
\begin{aligned}
& \left|H_{p w m}(f)\right| \\
& =\sqrt{2 \frac{\cos \left(\omega(d-1) T_{s}\right)+\cos \left(\omega d T_{s}\right)-2}{\cos \left(\omega T_{s}\right)-1}-1} .
\end{aligned}
$$

This transfer function is illustrated in Fig. 7(a) for several values of $d$. (Note that 0.5 on the $\mathrm{x}-$ axis is the Nyquist frequency $f_{N}$.) The values for $d$ were chosen to show the range of possible equalizer gains. In Fig. 8(a), the same function is given with the y-axis in dBs. It can be seen from this figure that a duty-cycle closer to $50 \%$ results in a steeper transfer function. Changing the duty-cycle from $100 \%$ to $50 \%$ attenuates the low-frequency components of the pulse spectrum as compared to the spectrum of a polar NRZ pulse. The result is pre-emphasis filtering. The next subsection shows how this transfer function compares to that of the 2-tap SSF.

\subsubsection{PSD and transfer function of 2-tap SSF}

The function $\left|H_{p w m}(f)\right|$ is now compared to the modulus of the transfer function $\left|H_{f i r}(f)\right|$ of the 2-tap SSF. In Fig. 1(b), TX waveforms for a 2-tap SSF-PE filter are shown. The 2-tap FIR equalized pulse $p_{\text {fir }}(t)$, shown in Fig. 4(b), is defined as follows:

$$
p_{f i r}(t)=\left\{\begin{array}{cc}
0, & t<-T_{s}, \\
c_{1}, & -T_{s} \leq t<0, \\
c_{2}, & 0 \leq t<T_{s}, \\
0, & T_{s} \leq t,
\end{array}\right.
$$

where $c_{1}$ and $c_{2}$ denote the values of the first and the second FIR taps respectively, and $T_{s}$ again denotes the symbol duration. A good fit to copper cables is obtained by choosing $c_{1}>0$ and $c_{2}<0$, while $\left|c_{1}\right|>\left|c_{2}\right|$. The sum of the absolute values of all tap weights has to be limited to a certain value (at or below the supply voltage) to avoid compression at the TX output. When the output swing is again normalized to $+/-1 \mathrm{~V}$, like in the previous subsection, and the whole of the available swing is used, $c_{1}$ and $c_{2}$ have to comply with:

$$
\left|c_{1}\right|+\left|c_{2}\right|=1
$$

These coefficients can be rewritten as $c_{1}=r$ and $c_{2}=r$-1, with one coefficient $r$, chosen from the interval $[0.5,1]$, that completely controls the shape of the filter transfer function. Function $p_{\text {fir }}(t)$ now becomes:

$$
p_{\text {fir }}(t)=\left\{\begin{array}{cc}
0, & t<-T_{s}, \\
r, & -T_{s} \leq t<0, \\
r-1, & 0 \leq t<T_{s}, \\
0, & T_{s} \leq t .
\end{array}\right.
$$

As mentioned earlier, in practice, this coefficient can be determined automatically using a control loop. 
The spectrum $P_{f i r}(f)$ of the PWM pulse is calculated by taking the Fourier transform of $p_{f i r}(t)$ :

$$
P_{f i r}(f)=\int_{-\infty}^{\infty} p_{f i r}(t) e^{-j \omega t} d t=\int_{-T_{s}}^{0} r e^{-j \omega t} d t+\int_{0}^{T_{s}}(r-1) e^{-j \omega t} d t .
$$

Simplifying leads to:

$$
P_{f i r}(f)=\frac{1}{j \omega}\left(r e^{j \omega T_{s}}+(1-r) e^{-j \omega T_{s}}-1\right) .
$$

Now we calculate the power spectral density $P S D_{F I R}$ for the 2-tap SSF filter:

$$
\begin{aligned}
& P_{f i r}(\omega)=\frac{\left|P_{f i r}(\omega)\right|^{2}}{T_{s}} \sum_{k=-\infty}^{k=\infty} R(k) e^{j \omega k T_{s}} \\
& =2 \frac{\left(r^{2}-r\right)\left(1-\cos \left(2 \omega T_{s}\right)\right)-\cos \left(\omega T_{s}\right)+1}{\omega^{2} T_{s}} .
\end{aligned}
$$

This function is shown in Fig. 6. Note that the situation with $r=1$ corresponds to normal polar NRZ line coding.

The transfer function $H_{f i r}(f)$ of the 2-tap SSF can be found by dividing $P_{f i r}$ over $P_{N R Z}$, resulting in:

$$
H_{f i r}(f)=\frac{1}{2} \frac{r e^{j \omega T_{s}}-(r-1) e^{-j \omega T_{s}}-1}{j \sin \left(\omega T_{s} / 2\right)}
$$

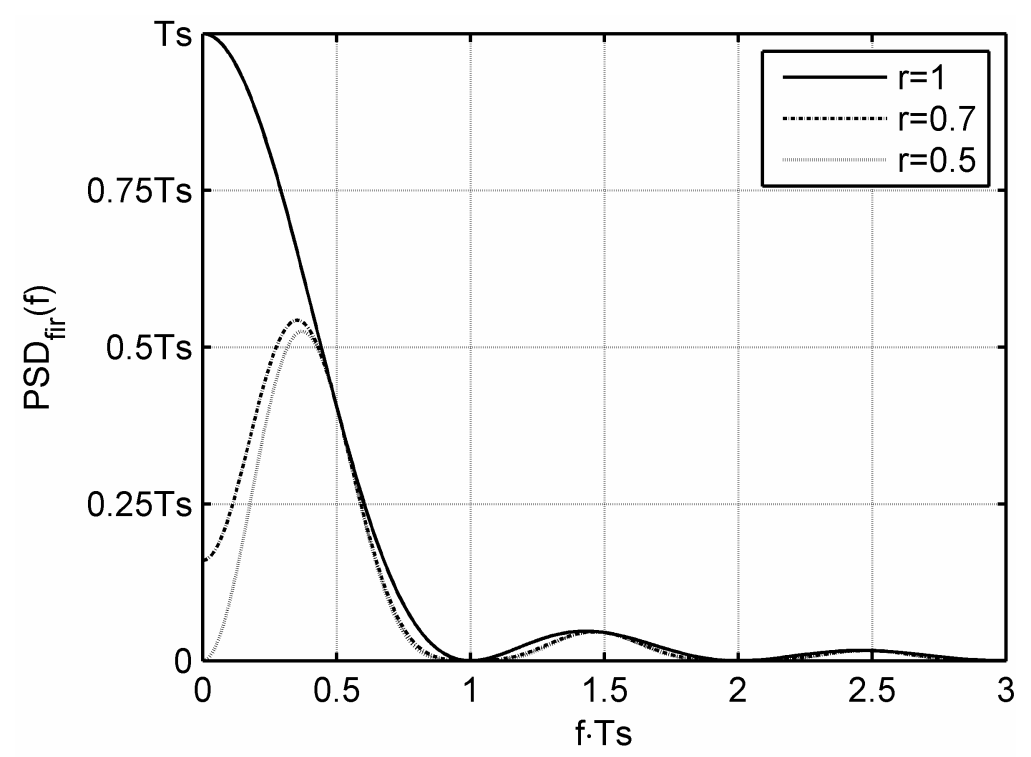

Fig. 6. Power spectral densities for the FIR signal. 
Taking the modulus yields:

$$
\left|H_{f i r}(f)\right|=\sqrt{\left(r^{2}-r\right) \frac{\cos \left(2 \omega T_{s}\right)-1}{\cos \left(\omega T_{s}\right)-1}+1} .
$$

This function is illustrated in Fig. 7(b) for several values of $r$. The values for $r$ were chosen to show the range of possible equalizer gains. Fig. 8(b) illustrates the same function with the yaxis in $\mathrm{dBs}$. The closer $r$ is to 0.5 , the more low-frequency attenuation the filter exhibits.

\subsubsection{Comparison of transfer functions}

We now try to understand what makes the PWM filter fit better to the channel transfer function than the 2-tap SSF filter. We know that this must be the case because of the higher measured loss compensation, as is shown later.

Comparing the PWM and 2-tap SSF transfer functions (Fig. 7(a) / 8(a) and Fig. 7(b) / 8(b) respectively), it can be seen that in the low frequency (LF) range the PWM-PE filter behaves like the 2-tap SSF filter (first order behavior). However in the high frequency (HF) range, at frequencies just below $f_{N}$, the PWM filter behaves differently. Due to the time-varying behavior of the PWM filter, near $2 f_{N}$ the effective gain of the PWM filter increases to infinity. As a result, at $f_{N}$ (at 0.5 on the x-axis) the calculated magnitude of the PWM filter transfer function has a value of one while its derivative is non-zero, unlike with the 2-tap SSF filter. The PWM filter transfer function can be seen as a higher order filter with only one parameter.

While both filters have a different slope at $f_{N}$, their transfer at $f_{N}$ is equal: both filters leave the amplitude of the fastest data transitions ('101010') unchanged. This is precisely what a preemphasis filter for these channels should do. In fact, de-emphasis is a better word. Ideally, the equalizer should attenuate all frequencies below $f_{N}$ and leave the fastest transitions untouched. This is because the channel has an attenuation that increases monotonically with frequency, as is shown in chapter two. The cable loss at $f_{N}$ therefore roughly determines the eye height at the cable output. This eye height is approximately the same for both filters up to $\sim 20 \mathrm{~dB}$ cable loss at $f_{N}$. After that, the eye of the 2-tap SSF closes and PWM-PE still has an open eye, up to $\sim 30 \mathrm{~dB}$, as is shown later.

Finally, one could ask whether PWM filtering is essentially the same as 2-tap half-symbolspaced FIR filtering. The modulus of its transfer function $\left|H_{f f} f(f)\right|$ is the same as that for 2-tap SSF, except that $\omega$ is replaced by $\omega / 2$ :

$$
\left|H_{f f f}(f)\right|=\sqrt{\left(r^{2}-r\right) \frac{\cos \left(\omega T_{s}\right)-1}{\cos \left(\omega T_{s} / 2\right)-1}+1} .
$$

This is shown in Fig. 7(c), and with the y-axis in dB in Fig. 8(c). The 2-tap half-symbolspaced filter has a frequency response two times wider than its 2-tap SSF equivalent. However, as a result of that, the transfer function is stretched out and is not as steep as that of the PWM-PE filter in the frequency range from zero to $f_{N}$, in turn resulting in a lower loss compensation. Whereas the PWM and 2-tap SSF filters attenuate only the signals below $f_{N}$, a 2-tap half-symbol-spaced FIR filter also attenuates the signals at $f_{N}$. Half-symbol-spaced FIR filters are sometimes used at the receiver side [Farjad-Rad]. 


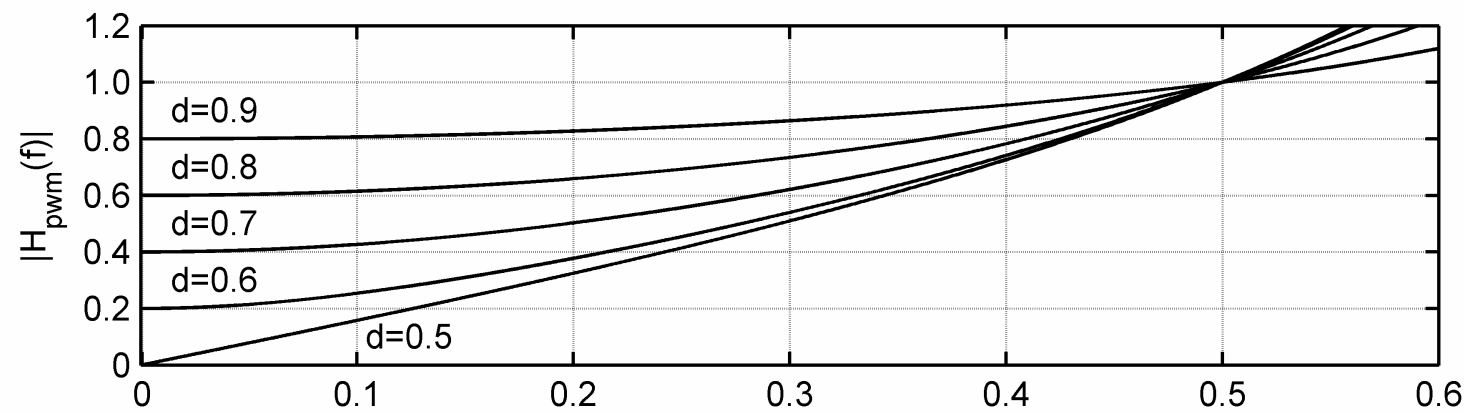

(a)

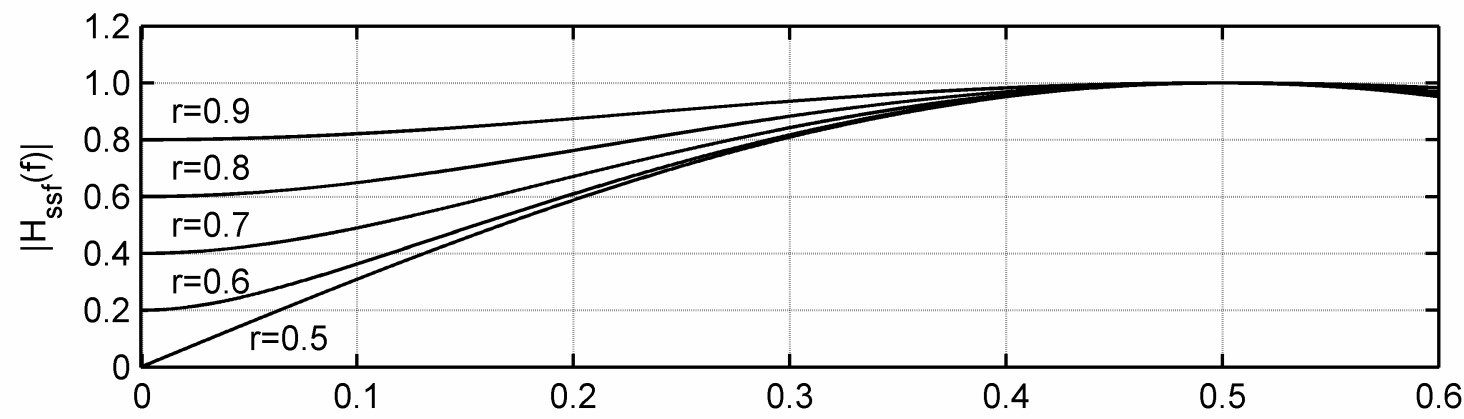

(b)

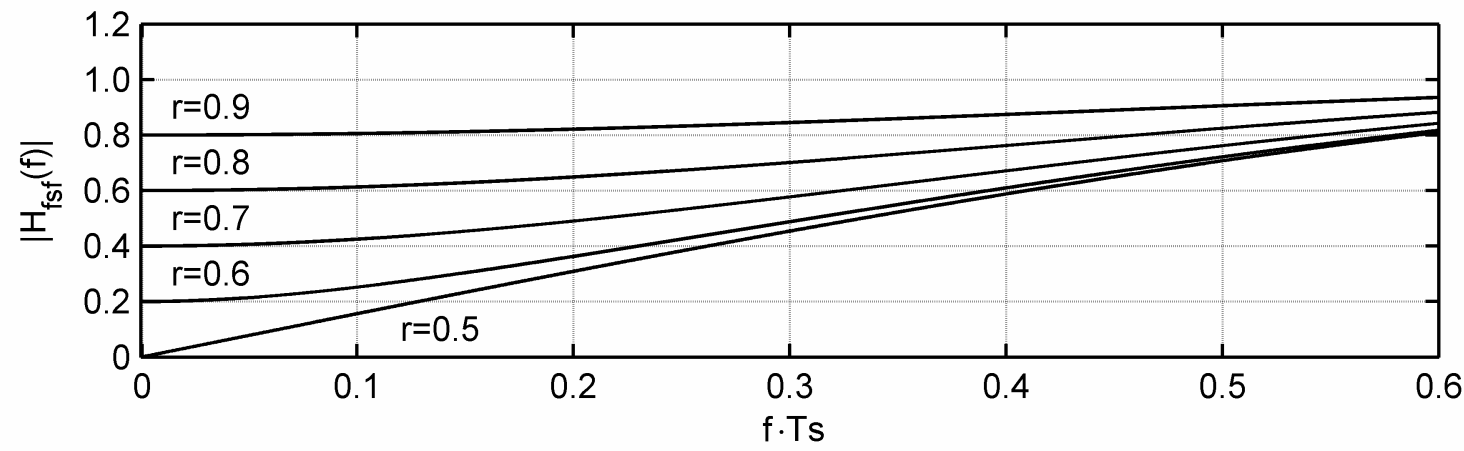

(c)

Fig. 7. Calculated magnitude of filter transfer. Note that $f_{N}$ is (per definition) at 0.5 on the $\mathrm{X}$ axis. (a). PWM-PE filter. (b). 2-tap SSF-PE filter. (c) 2-tap HSF-PE filter. 


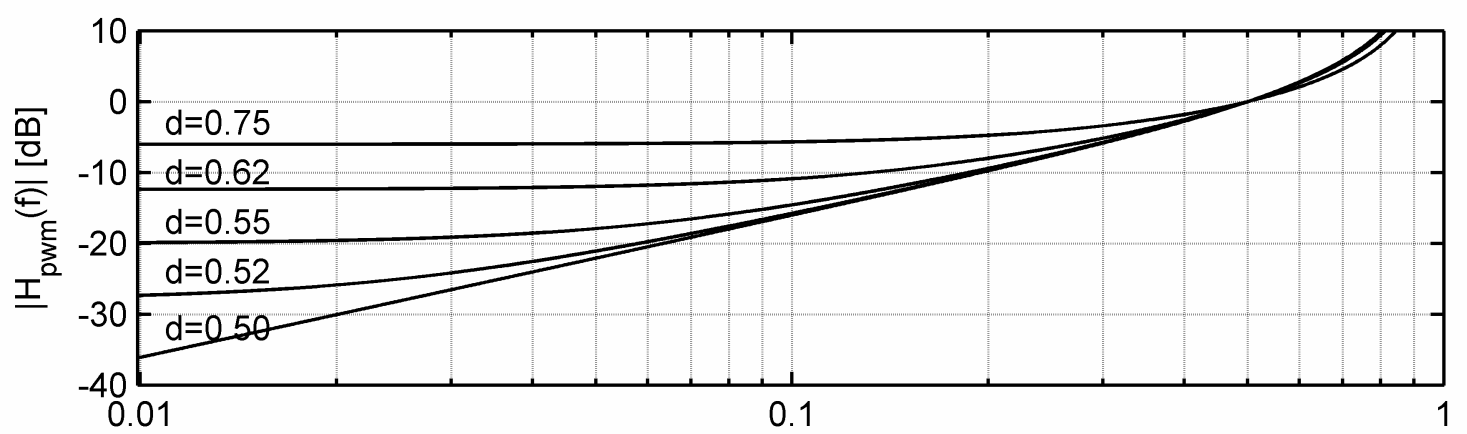

(a)

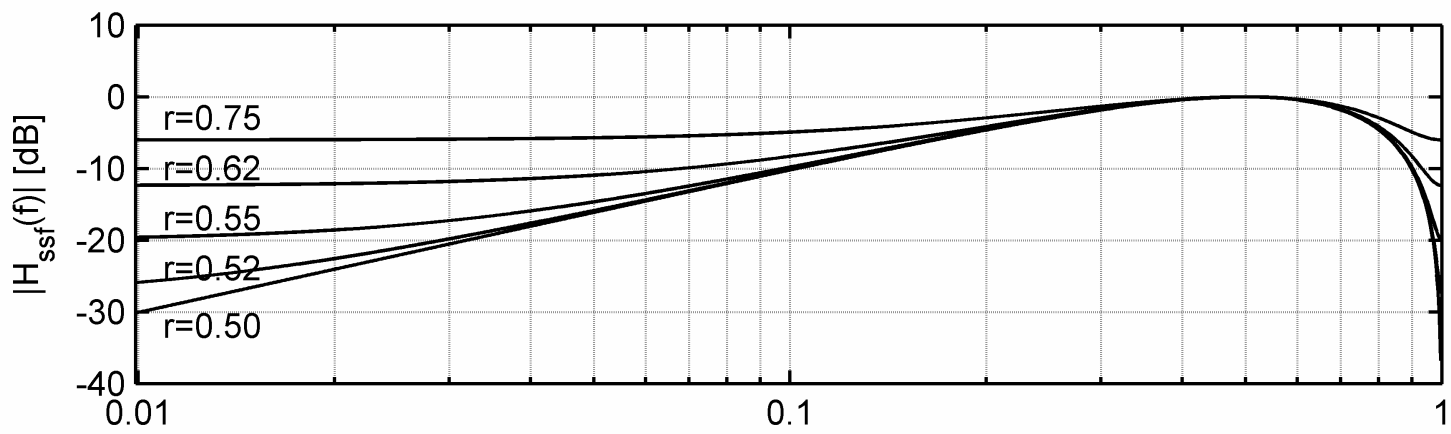

(b)

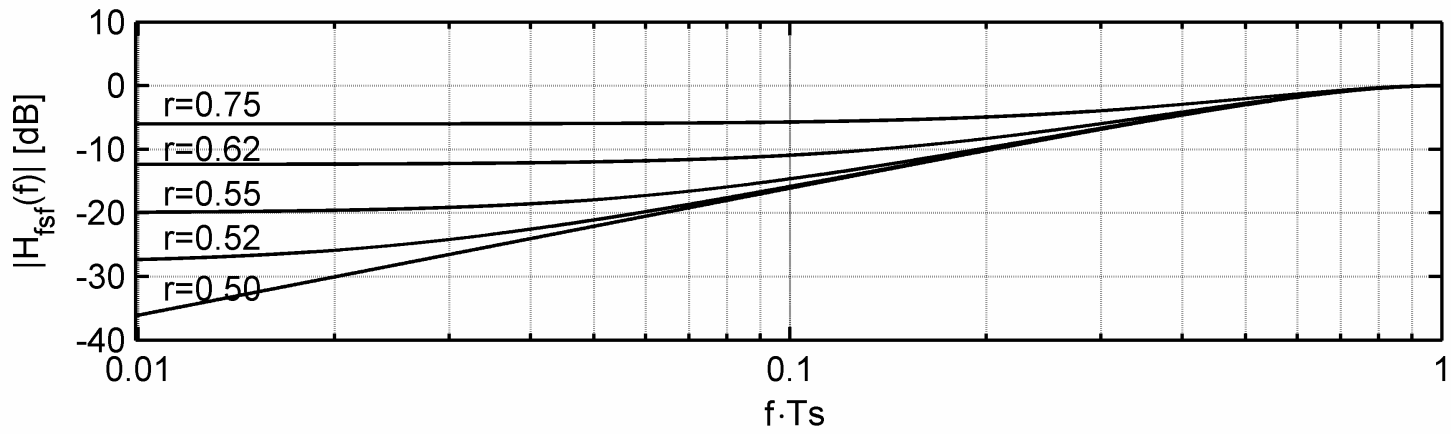

(c)

Fig. 8. Calculated magnitude of filter transfer, y-axis in $\mathrm{dB}$. Note that $f_{N}$ is (per definition) at 0.5 on the X-axis. (a). PWM-PE filter. (b). 2-tap SSF-PE filter. (c) 2-tap HSF-PE filter. 


\subsubsection{Equalized channel channel transfer function}

Next, we calculate the equalized channel transfer function for the 2-tap FIR filters and the PWM filter. A theoretical first-order channel can be perfectly equalized with PWM preemphasis as calculated in [Schinkel-2]. A cable does not have a first order transfer function but still can be equalized with PWM pre-emphasis, and better than with 2-tap SSF, as is shown later. The equalized transfer function is calculated by taking the measured cable transfer of $25 \mathrm{~m} \mathrm{RG}-58 \mathrm{CU}$ cable and multiplying it with the calculated theoretical transfer of the pre-emphasis filters. A bit length of $T_{s}=200 \mathrm{ps}$ is chosen. The results are shown in Fig. 9. The values for $r$ and $d$ are chosen around the values that minimize the peak distortion. (See section 4.4.) We can now determine the flatness in the frequency interval $\left[0, f_{N}\right]$. Again, note that $f_{N}$ is at 0.5 on the $\mathrm{x}$-axis. The channel response for PWM pre-emphasis is flat to within $5 \mathrm{~dB}$ (Fig. 9(a)), while the FIR response is only flat to within 10dB, shown in Fig. 9(b). PWMPE clearly outperforms FIR-PE. For shorter cable lengths the responses become flatter. The half-symbol spaced 2-tap FIR is shown in Fig. 9(c); it is flat only to within 8dB.

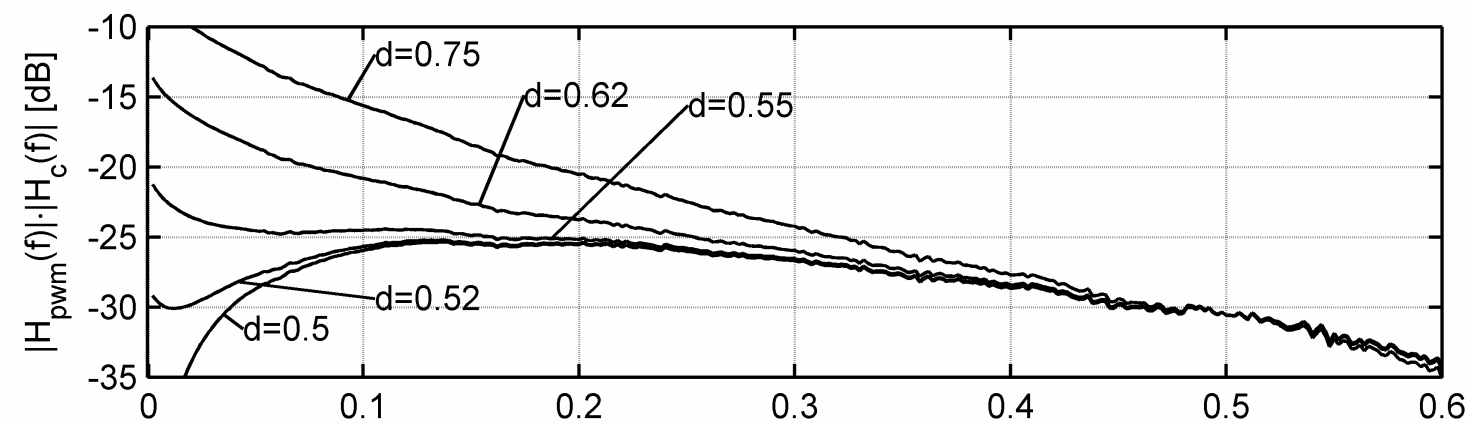

(a)

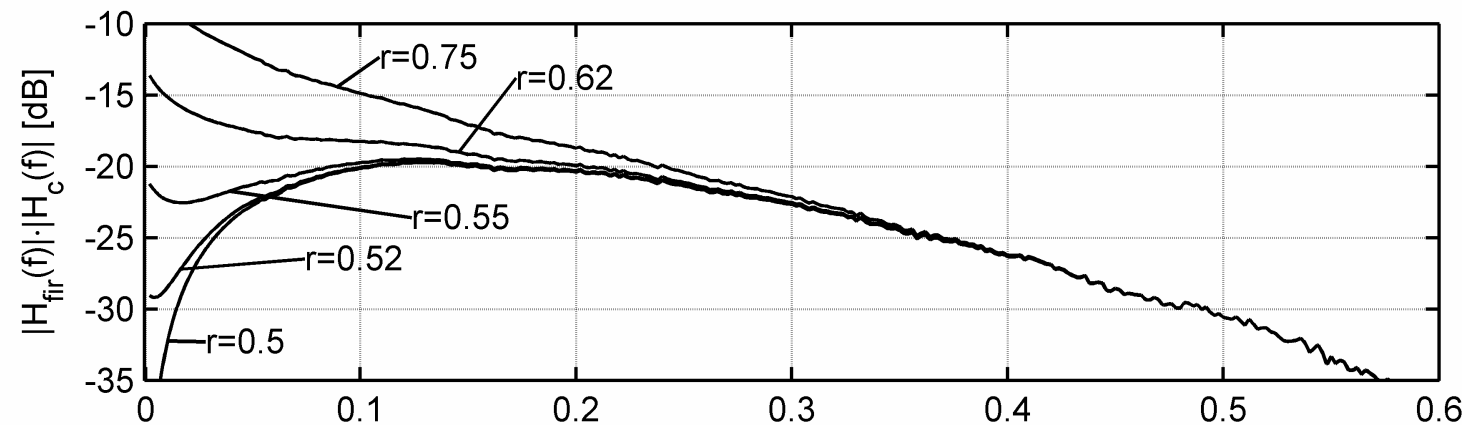

(b)

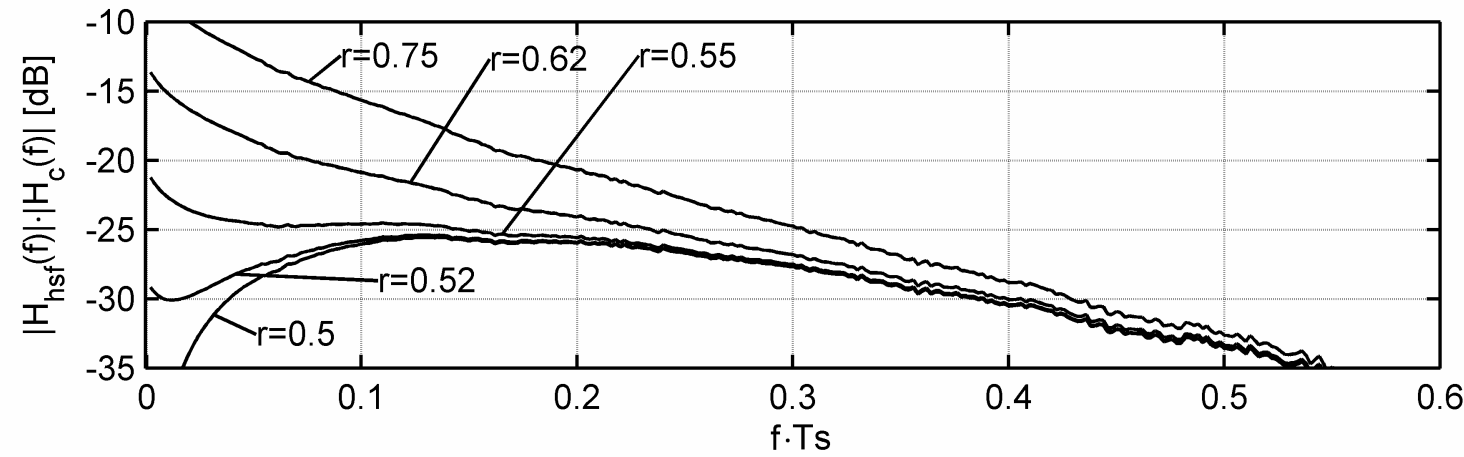

(c)

Fig. 9. Equalized transfer of $25 \mathrm{~m} \mathrm{RG}-58 \mathrm{CU} . H_{c}(f)$ indicates the measured transfer function of 25m RG-58CU. (a). PWM. (b). 2-tap SSF. (c) 2-tap HSF. 


\subsubsection{Filter DC behavior: move power to higher frequencies}

It is important to know how the output of the PWM filter differs from that of the SSF filter when transmitting many $1 \mathrm{~s}$ in succession, and when transmitting a quickly alternating pattern of $1 \mathrm{~s}$ and $0 \mathrm{~s}$. Using a simple Fourier series calculation, we calculate the spectrum for the LF pattern $\{. ., 1,1,1,1, .$.$\} . Next, we calculate the spectrum for the HF pattern \{. ., 1,-1,1,-1, .$.$\} and$ compare the two. We then do the same for the 2-tap SSF filter.

The Fourier coefficients for a periodic waveform are calculated as follows:

$$
c_{n}=\frac{1}{T_{0}} \int_{0}^{T_{0}} y(t) e^{-j n 2 \pi t / T_{0}} d t
$$

and the waveform then can be reconstructed using:

$$
y(t)=\sum_{n=-\infty}^{\infty} c_{n} e^{j n 2 \pi t / T_{0}}
$$

For the PWM filter, we define two periodic waves, one for the LF pattern and one for the HF pattern. First, the LF $\{1,1,1,1, .$.$\} pattern y_{p w m, L F}(t)$ :

$$
y_{p w m, L F}(t)=\left\{\begin{array}{cc}
1, & 0 \leq t \bmod T_{s}<d T_{s}, \\
-1, & d T_{s} \leq t \bmod T_{s}<T_{s}
\end{array}\right.
$$

Next, the $\operatorname{HF}\{1,-1,1,-1, .$.$\} pattern y_{p w m, H F}(t)$ :

$$
y_{p w m, H F}(t)=\left\{\begin{array}{cc}
1, & 0 \leq t \bmod 2 T_{s}<d T_{s}, \\
-1, & d T_{s} \leq t \bmod 2 T_{s}<(d+1) T_{s}, \\
1, & (d+1) T_{s} \leq t \bmod 2 T_{s}<2 T_{s} .
\end{array}\right.
$$

Examples are given in Fig. 10(c) and 10(f), for $d=0.7$. For the 2-tap SSF filter, we again define two periodic waves, one for the LF pattern and one for the HF pattern. First, the LF $\{1,1,1,1, .$.$\} pattern y_{f i r, L F}(t)$ :

$$
y_{f i r, L F}(t)=r-(1-r)=2 r-1
$$

Next, the $\operatorname{HF}\{1,-1,1,-1, .$.$\} pattern y_{f i r, H F}(t)$ :

$$
y_{f i r, H F}(t)=\left\{\begin{array}{cc}
1, & 0 \leq t \bmod 2 T_{s}<T_{s}, \\
-1, & T_{s} \leq t \bmod 2 T_{s}<2 T_{s} .
\end{array}\right.
$$

Examples are given in Fig. 10(b) and 10(e), for $r=0.7$. We choose $d$ and $r$ to be equal because then the DC levels of the outputs are equal for both filters.

In 10(a) and 10(d), the non-equalized polar NRZ patterns are shown. 


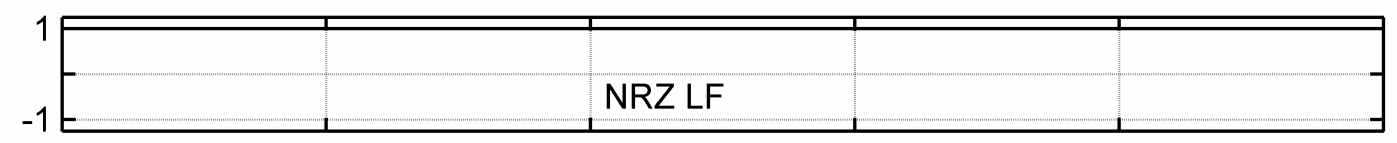

(a)

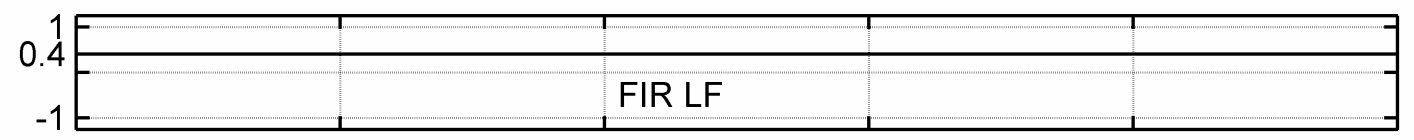

(b)

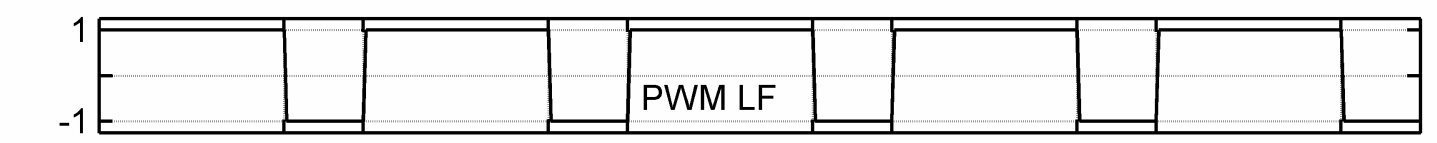

亏

(c)

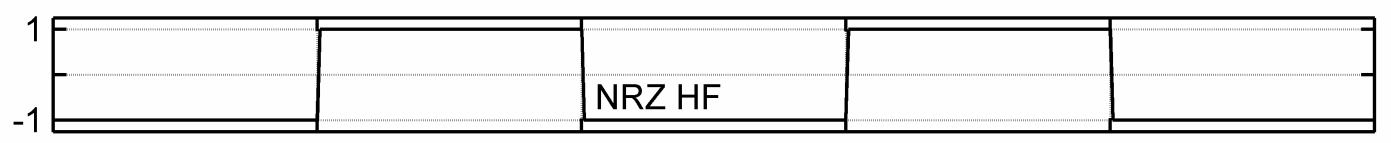

(d)

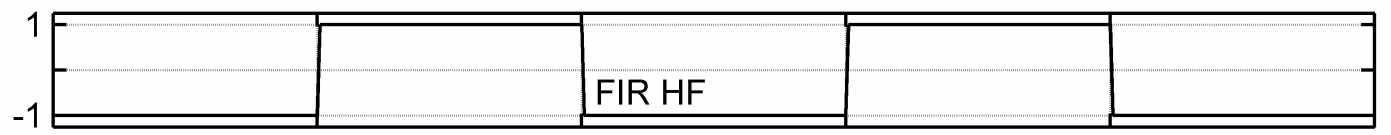

(e)

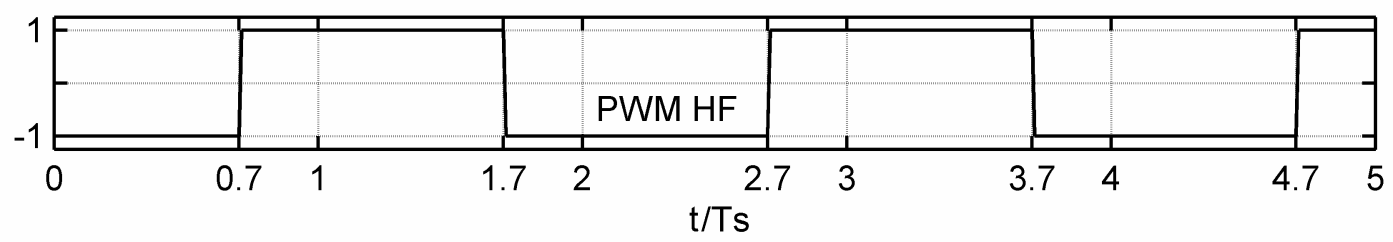

(f)

Fig. 10. LF and HF patterns. (a), (b) and (c) LF patterns for NRZ, FIR and PWM respectively. (d), (e) and (f) HF patterns for NRZ, FIR and PWM respectively.

Note that the repetition period needs to be chosen as $T_{0}=2 T_{s}$. Note also that $n=1$ corresponds to the Nyquist frequency $f_{N}$. Now the coefficients can be calculated using Eq. 21. For the PWM LF wave, the results are:

$$
c_{n, P W M, L F}=\left\{\begin{array}{cc}
2 d-1, & n=0, \\
\frac{j}{n \pi}\left(e^{-j 2 \pi n d}-1\right), & n \neq 0 .
\end{array}\right.
$$

Note that all coefficients are dependent on $d$. For $\mathrm{n}=0$, the magnitude is dependent on $d$. For $\mathrm{n} \neq 0$, both magnitude and phase are dependent on $d$. The magnitude of the coefficients is plotted in Fig. 11(a). 


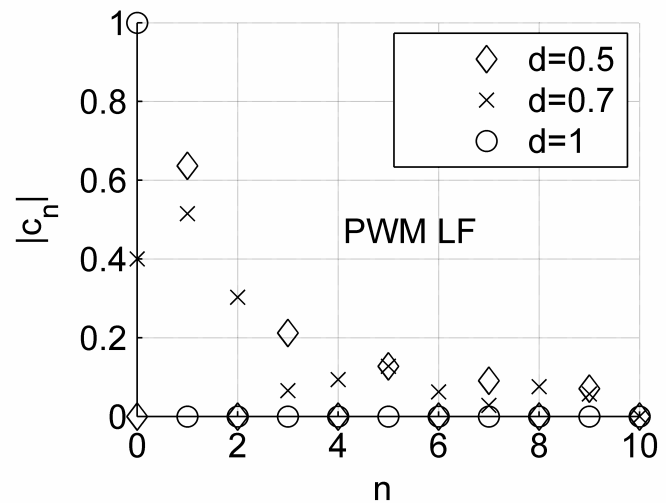

(a)

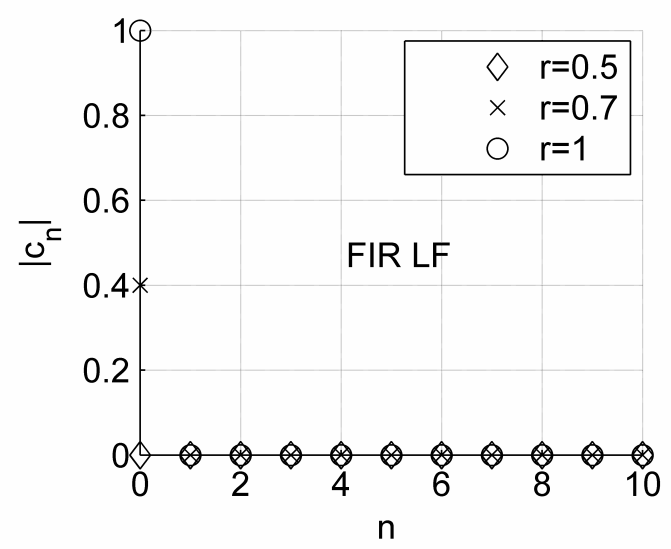

(c)

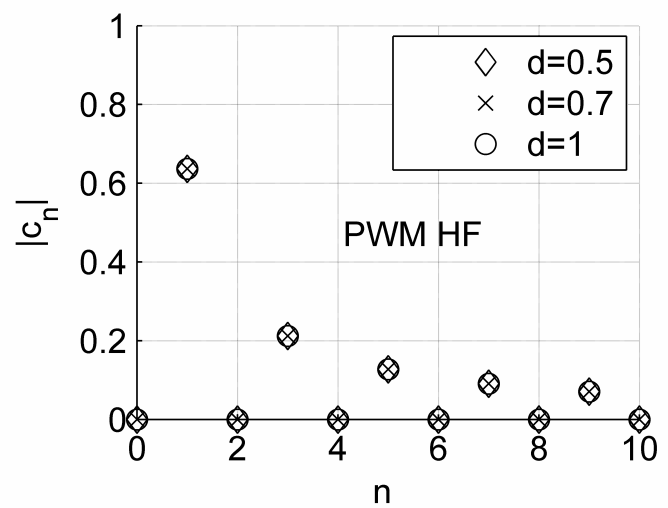

(b)

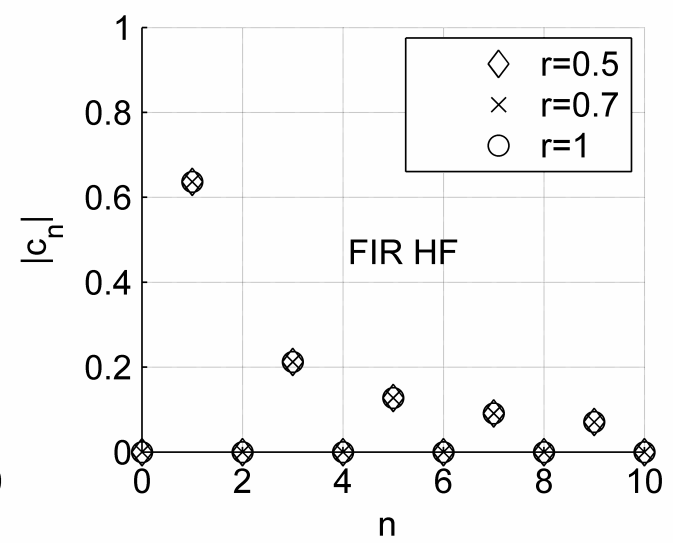

(d)

Fig. 11. Magnitude $\left|c_{n}\right|$ of Fourier coefficients. (a) PWM LF. (b) PWM HF. (c) FIR LF. (d) FIR HF.

For the PWM HF wave, the results are:

$$
c_{n, P W M, H F}=\left\{\begin{array}{cc}
0, & n=0, \\
\frac{2 j}{n \pi} e^{-j \pi n d}, & n=\text { odd }, \\
0, & n=\text { even } .
\end{array}\right.
$$

These coefficients are simply equal to the coefficients of a (phase-shifted) square wave. Note that the phase (time shift) is dependent on $d$. The magnitude of the coefficients is illustrated in Fig. 11(b).

For the FIR filter, we also calculate the Fourier series coefficients. The results for the LF FIR wave are:

$$
c_{n, f i r, L F}=\left\{\begin{array}{cc}
2 r-1, & n=0, \\
0, & n \neq 0 .
\end{array}\right.
$$

Only for $n=0$ are the coefficients nonzero. This is illustrated in Fig. 11(c). 
For the FIR HF wave, the results are:

$$
c_{n, f i r, H F}=\left\{\begin{array}{cc}
0, & n=0, \\
\frac{-2 j}{n \pi}, & n=\text { odd }, \\
0, & n=\text { even } .
\end{array}\right.
$$

This is the same as for the PWM HF wave, except for a phase shift. Note that there is no dependency on $r$ whatsoever. The magnitude of the coefficients is shown in Fig. 11(d).

In conclusion, the difference between PWM and 2-tap SSF is as follows. For the quickly alternating $\{1,-1,1,-1,1,-1\}$ waves, there is only a difference in phase (time shift). Neither filter attenuates the HF pattern. This is the required behavior for a de-emphasis filter that needs to compensate a monotonically decreasing cable transfer function, as mentioned in subsection 4.3.3. For the $\operatorname{LF}\{1,1,1,1,1,1\}$ pattern, the behavior is different. A 2-tap SSF outputs less power for lower switching frequencies. Where the 2-tap SSF is decreasing the output power for low frequency output patterns, the PWM filter instead outputs the same power as for its HF pattern, because the absolute squared output voltage remains the same (see Fig. 10). Instead of decreasing the power, the PWM filter transfers that power to higher frequencies, where it is dissipated by the cable.

Could the additional HF noise, generated by the PWM filter, become a cause of crosstalk? Near-end crosstalk (NEXT), from the transmitter to a nearby, sensitive receiver, could become especially problematic. A possible solution is as follows. Most of this HF power is just dissipated by the cable, so we might as well filter this out before it leaves the transmitter, for example by using an (adjustable) low-pass filter at the TX output. As long as this low-pass filter has a pole that is well above the dominant pole in the rest of the channel, crosstalk can be effectively decreased without harming the equalization. The pole of this low-pass filter can be located at the Nyquist frequency $f_{N}$.

\subsection{Numerical time-domain simulations of ISI}

In the previous section, it was shown from a frequency domain perspective that PWM-PE provides more relative HF boost than 2-tap SSF. In this section, the two pre-emphasis methods are compared from a time-domain perspective by calculating the remaining ISI at the output of the channel as a function of the bit rate. We calculate the peak distortion to obtain an indication of the eye opening. The goal of the simulations is to find the maximum achievable bit rate for the pre-emphasis filters at which the remaining ISI is still acceptable.

The following approach is used. First, the channel responses to the pre-emphasis pulses $p_{p w m}(t)$ and $p_{\text {fir }}(t)$ are calculated for a number of bit rates, at the optimum pre-emphasis setting (subsection 4.4.1). Next, at these points, the remaining ISI in the received signal is calculated as a function of pre-emphasis setting, symbol length, and channel time constant using a peak distortion analysis. The remaining ISI is then plotted and compared between the two different equalizers (subsection 4.4.2). Finally, the sensitivity to jitter of the PWM pre-emphasis scheme is analyzed in subsection 4.4.3. 


\subsubsection{Model of skin-effect-only channel}

To calculate the time domain channel response, the skin-effect impulse response $h_{l}(t)$ is used, as described in chapter 2, subsection 2.4.1:

$$
h_{1}(t)=\frac{\sqrt{\tau_{1}}}{2 t \sqrt{\pi t}} \cdot e^{-\frac{\tau_{1}}{4 t}}
$$

where $\tau_{1}$ is a channel time constant. In Fig. 12, $h_{1}(t)$ is shown. The $\mathrm{x}$-axis shows time divided by $\tau_{l}$. The y-axis shows $h_{l}(t) \cdot \tau_{l}$. The axes are chosen in this way to clearly show the maxima and time span of the functions. It can be seen that $h_{1}(t)$ is asymmetrical over time with a very long tail.

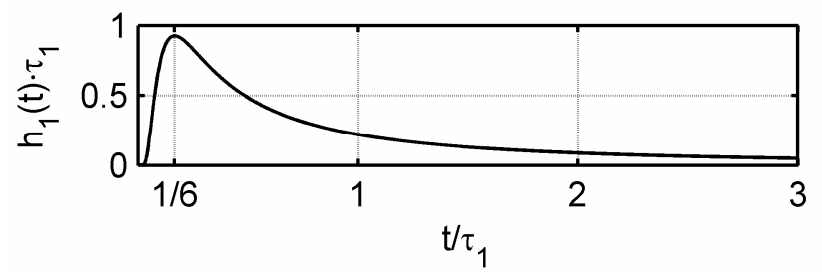

Fig. 12. Skin-effect impulse response $h_{1}(t)$.

We use this skin-effect-only channel as a good approximation of the response of many types of cable, as these are mostly skin-effect dominated. Also, to an extent, the dielectric impulse response has a similarly asymmetrical shape ${ }^{1}$. See Chapter 2 for a more detailed estimation of the accuracy of this simplification.

The Fourier transform of Eq. 31 gives us the frequency domain transfer function $H(j \omega)$ for the skin-effect:

$$
H(j \omega)=e^{-\sqrt{j \omega \tau_{1}}}
$$

The magnitude of this transfer function at the Nyquist frequency $f_{N}$ for a skin-effect-only channel can be calculated by substituting $\omega=2 \pi f_{N}=\pi / T s$ in Eq. 32 and taking the magnitude:

$$
\left|H\left(f_{N}\right)\right|=\left|e^{-\sqrt{j \pi \frac{\tau_{1}}{T_{s}}}}\right|=e^{-\frac{1}{2} \sqrt{2 \pi \frac{\tau_{1}}{T_{s}}}} .
$$

Note that $\left|H\left(f_{N}\right)\right|$ is solely dependent on the ratio $T_{s} / \tau_{1}$. The dependence is illustrated in Fig. 13.

\footnotetext{
${ }^{1}$ When writing our paper [Schrader-6] we were not yet aware of recent advances in dielectric loss modeling and we used an inaccurate model [vdPlaats] for the time-domain simulation of dielectric loss. Thus a symmetrical impulse response was used, while - on the contrary - we should have used an asymmetrical one, much like the skin effect impulse response. (See also Chapter 2). As a consequence, we were led to the conclusion that the PWM equalization method would not work so well on channels dominated by dielectric loss. As we now know, this was far too pessimistic. In this chapter, we will show with measurements that the PWM equalization method can indeed provide $25 \mathrm{~dB}$ loss compensation on an FR4 PCB trace.
} 


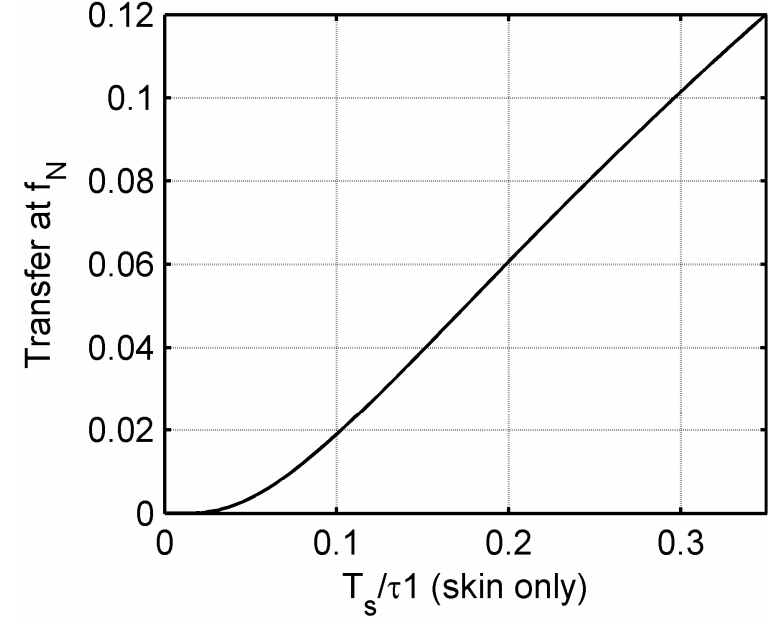

(a)

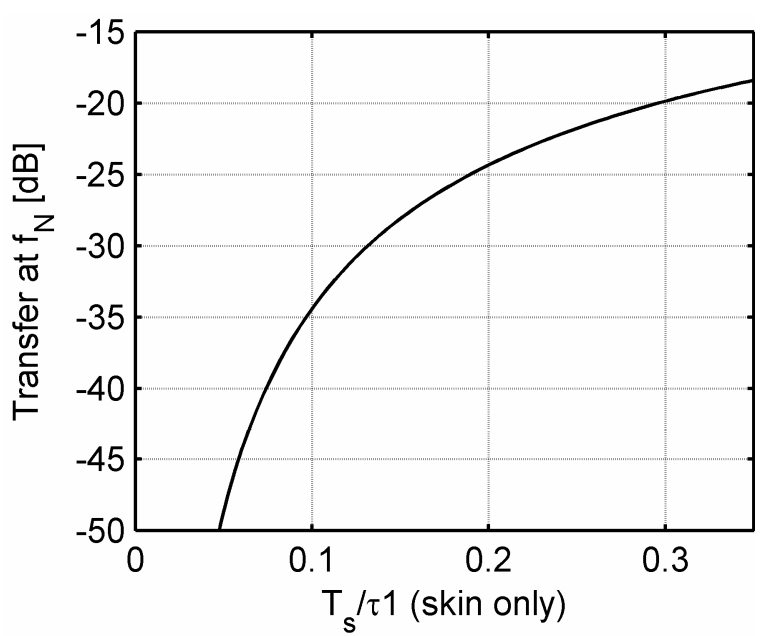

(b)

Fig. 13. Magnitude of transfer function at $f_{N}$ of skin-only channel, as a function of $T_{s} / \tau_{1}$.

(a) Linear. (b) In dBs.

\subsubsection{Peak distortion analysis}

A theoretical channel with only skin loss and no dielectric loss $\left(\tau_{1} \neq 0, \tau_{2}=0\right)$ is analyzed. The response $y_{\text {pwm }}(t)$ of the channel to a single PWM pulse is calculated as follows:

$$
y_{p w m}(t)=p_{p w m}(t) * h_{c}(t)
$$

where the asterisk denotes the convolution operation. To quantify the level of remaining ISI, the peak distortion is calculated, which is defined as [Lucky]:

$$
D s_{p e a k, I S I}=\frac{1}{\left|y_{p w m}\left(t_{s}\right)\right|} \sum_{\substack{n=-\infty \\ n \neq 0}}^{n=\infty}\left|y_{p w m}\left(t_{s}+n T_{s}\right)\right|,
$$

where $t_{s}$ is the sample moment. For example, a value of $D s_{p e a k, I S I}=0.2$ means that the eye diagram for the worst-case data pattern is $20 \%$ closed. In a simple receiver with a bang-bang phase detector, the received signal's median zero crossing is used for time reference, and then data is sampled at a fixed time, generally $T_{s} / 2$, away from the zero crossing. After that, the ISI contributions can be found at distances of $n \cdot T_{s}$ from $t_{s}$. The peak distortion $D s_{\text {peak,ISI }}$ is a function of the symbol length $T_{s}$, the channel time constant $\tau_{l}$, the sample moment $t_{s}$ and the duty-cycle $d$ (or parameter $r$ for the SSF filter).

A reduction in the number of variables can be made by acknowledging that $D s_{\text {peak,ISI }}$ only depends on the ratio $T_{s} / \tau_{1}$. A large value for $T_{s} / \tau_{l}$ means that the bit rate is very low compared to the channel speed, whereas a small value means the opposite. The expectation is that for very small ratios the ISI becomes unacceptably large, whereas for very large ratios it converges to zero.

The mathematics involved in the manipulation of Eqs. 34 and 35 is rather complicated and does not yield concise symbolic results. Therefore, we resort to numerical computation. In the simulation, the zero-forcing criterion is applied to $D s_{p e a k, I S I}$ to find the optimum duty-cycle setting $d_{\text {opt }}$ (and the optimum FIR filter parameter $r$ ). 
In Fig. 14(a), the value of $D s_{\text {peak,ISI }}$ for $d_{\text {opt }}$ is plotted versus $T_{s} / \tau_{1}$, and in Fig. 14(b) $d_{\text {opt }}$ is plotted versus $T_{s} / \tau_{1}$. An identical procedure is followed for the 2-tap SSF PE filter. Again, the minimized $D s_{\text {peak,ISI }}$ for $r_{\text {opt }}$ for the SSF is plotted in Fig. 14(a), and the optimum SSF coefficient $r_{\text {opt }}$ is plotted in Fig 14(b) ${ }^{2}$.

Choosing $D s_{\text {peak }, I S I}=0.2$ for a reasonable eye opening, it can be seen from the figures that the FIR filter reaches this point at $T_{s} / \tau_{l}=0.19$ whereas the PWM filter reaches it at $T_{s} / \tau_{l}=0.09$. This means that the PWM-PE achieves twice the bit rate of the 2-tap SSF for the same peak distortion.

\subsubsection{Sensitivity to jitter}

Another point of interest is the sensitivity of PWM pre-emphasis to timing errors. An error in the duty-cycle will effectively change the pre-emphasis setting and thus have an effect on the eye opening at the receiver side. However, a certain error can be allowed in the duty-cycle setting.

Fig. 14(c) shows the width $W_{d}$ of the duty-cycle range wherein $D s_{\text {peak }, I S I}<0.2$. For the 2-tap SSF-PE filter, the same figure shows the width $W_{r}$ of the range of filter coefficient $r$ wherein $D s_{p e a k, I S I}<0.2$.

The y-axis in Fig. 14(c) is explained in the following example. It can be seen that $W_{d}=0.057$ at $T_{s} / \tau_{l}=0.3$, indicated in the figure by a ' + '. From Fig. 14(b), the optimum duty-cycle $d_{\text {opt }}=0.565$ indicates the middle of this range. This means that a duty-cycle from the interval $(0.537,0.594)$ will yield a peak distortion $D s_{p e a k, I S I}<0.2$. Next, for 2-tap SSF, from Fig. 14(c), $W_{r}=0.054$ at $T_{s} / \tau_{1}=0.3$, indicated in the figure by a circle. From Fig. 14(b), the optimum $r$ parameter $r_{\text {opt }}=0.610$ indicates the middle of this range. This means that a value for $r$ from the interval $(0.583,0.637)$ will yield a peak distortion $D s_{p e a k, I S K}<0.2$.

It can be seen that both $W_{d}$ and $W_{r}$ become smaller with increasing bit rate. The identical behavior shown in Fig. 14(c) demonstrates the interchangeability of timing and amplitude precision.

\footnotetext{
${ }^{2}$ Several different regions can be identified in the plots. In each region, another ISI term dominates the response (pre-cursor, $1^{\text {st }}$ post-cursor, $2^{\text {nd }}$ post-cursor, etc.).
} 

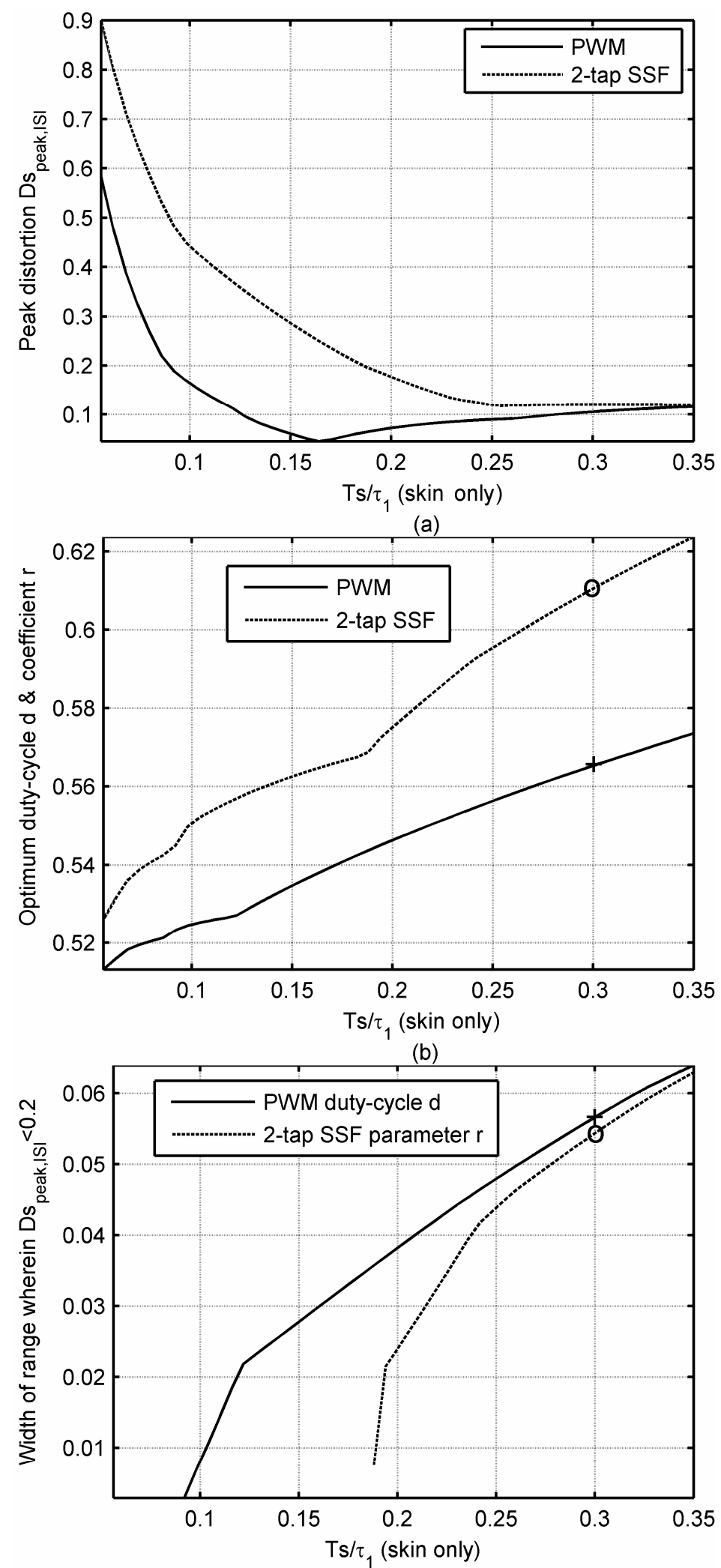

(c)

Fig. 14. (a) Simulated minimized peak distortion for skin channel. (b) Simulated optimum PWM duty-cycle and FIR coefficient for skin channel. (c) Simulated interval width for $D s_{\text {peak }, I S}<0.2$ of $d(\mathrm{PWM})$ and $r(2$-tap SSF). 


\subsection{PWM 1-edge chip design and measurements}

In this section, three proof-of-concept chip designs and their measured performance are presented. In subsection 4.5.1, we describe the first $0.13 \mu \mathrm{m}$ chip and give measurement results. Next, we describe the 90nm chip and give measurement results (subsection 4.5.2). Finally, in subsection 4.5 .3 , the second $0.13 \mu \mathrm{m}$ chip is described which was used to equalize a PCB trace.

All chips were designed using current-mode logic (CML) to provide maximum supply noise rejection and minimum supply noise injection and to keep timing noise as low as possible. A difference between up and down slew rates would have a negative effect on the fit of the preemphasis to the channel. The use of differential CML guarantees symmetrical up and down slew rates. An advantage over FIR pre-emphasis is that non-linear (symmetrical) slewing effects do not affect the equalizer's fit to the channel because only two signal levels are used. Moderate bandwidth limitations in the circuitry do not pose a problem because they will just become part of the total channel transfer function that needs to be compensated by the preemphasis.

\subsubsection{Chip 1: Design and Measurements}

In this subsection, the first $0.13 \mu \mathrm{m}$ chip is described. First, a circuit overview is given in 4.5.1.1. Next, in 4.5.1.2, the measurement results are given.

\subsubsection{Circuit overview}

The principle of operation of chip 1 is shown in Fig. 15(a). The data is XOR'ed with a pulsewidth modulated clock in order to provide pre-emphasized data. The PWM clock is generated using an OR gate and a delay circuit. The timing of the signals is illustrated in Fig. 15(b).
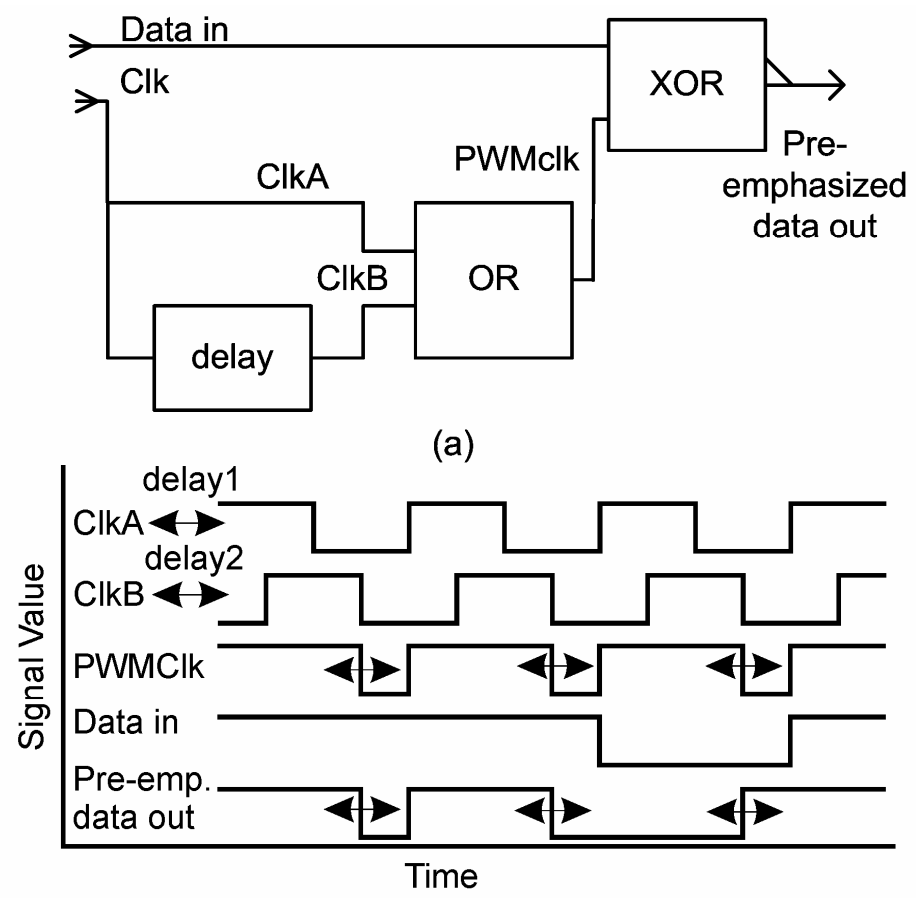

(b)

Fig. 15. (a). Circuit operation principle for chip 1. (b). Signals used in generating PWM signal. 


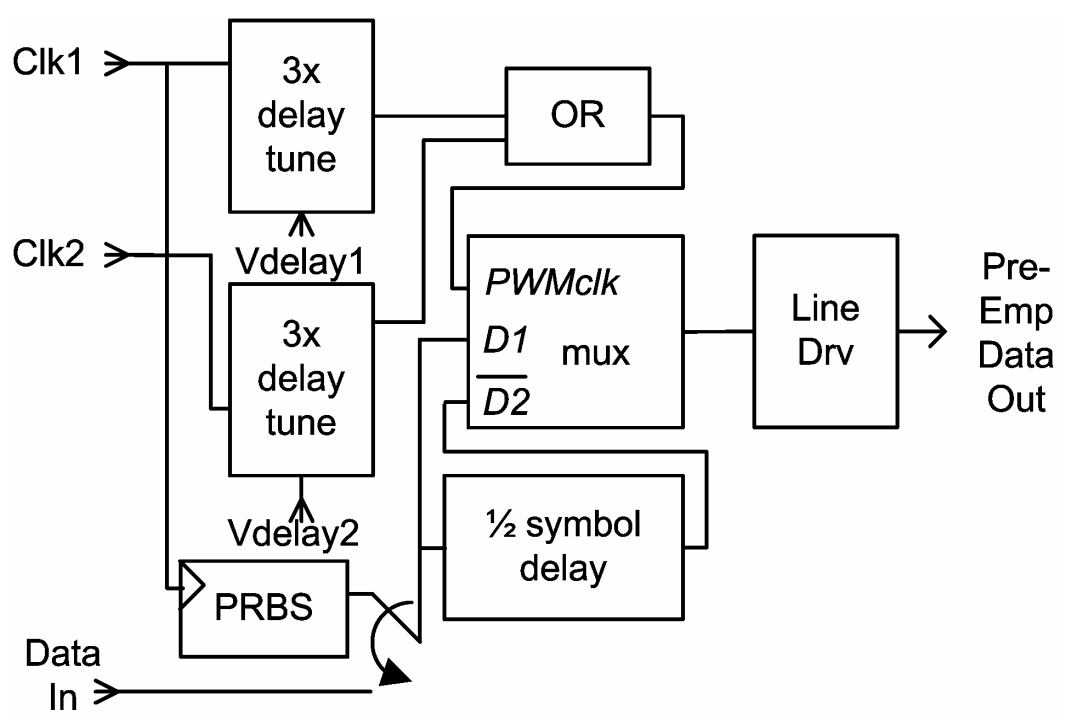

Fig. 16. Chip block diagram: PRBS generator, pre-emphasis circuit and line driver.

Fig. 16 shows the detailed block diagram. Because a small differential delay is more straightforward to generate than a short absolute delay, the relative delay for clock B is created by delaying $c l k 1$ by delay 1 and delaying $c l k 2$ by delay 2 . The differential delay is thus equal to (delay1-delay2) and can be controlled by voltage $V_{\text {delay } 1}-V_{\text {delay2 }}$. Both are differential voltages.

The XOR, shown in Fig. 17(a), is implemented using a multiplexer that selects either noninverted data $D 1$ or inverted data $D 2$. For an optimum timing margin, $D 2$ is delayed by half a symbol time using a negative edge clocked flip-flop [Schinkel-2]. The duty-cycle of the PWM pulse shape can be tuned between $50 \%-100 \%$, provided the relative phase-shift between clocks is adjustable between $0^{\circ}$ and $180^{\circ}$.

The time-shifted clock is generated using a variable delay circuit, illustrated in Fig. 17(b) [Razavi]. The delay between input and output of this circuit is mainly determined by the RC time at the output. By adding a positive feedback circuit in parallel to the output, which effectively behaves as a negative resistance, the effective $\mathrm{R}$ can be changed and hence the $\mathrm{RC}$-delay is changed. The value of the negative resistance is controlled by the differential delay control voltage $V_{\text {delay }}\left(=V_{\text {delP }}-V_{\text {delN }}\right)$, which divides the total bias current between the input differential pair and the negative resistance pair. For $\left.V_{\text {delP }}\right\rangle V_{\text {delN }}$, the delay is minimized. As the total bias current through the output resistors is fixed, the output swing remains constant. The required tuning range of the delay circuit depends on the desired symbol length and on the necessary duty-cycle range for pre-emphasis. The continuous tuning range can be enlarged by cascading multiple delay stages. Three stages are cascaded in our prototype to provide sufficient tuning range. For very large delay ranges this becomes impractical and it is more effective to combine the continuously tunable delay with discrete fixed delay steps. The prototype is designed to give the flexibility needed to evaluate the new PWM-concept in various ways. Therefore external clocks can be provided, for example to accommodate very low bit rates for long, low-quality cables. However, during normal operation, both inputs clk1 and $c l k 2$ (Fig. 16) can just be connected to the same clock. 


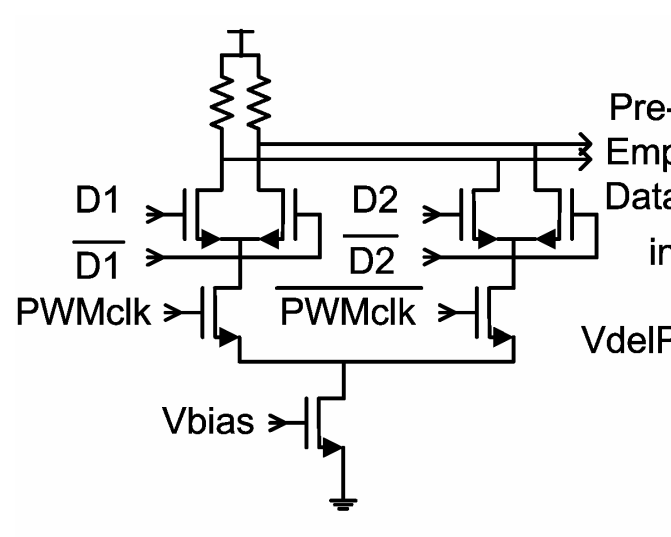

(a)

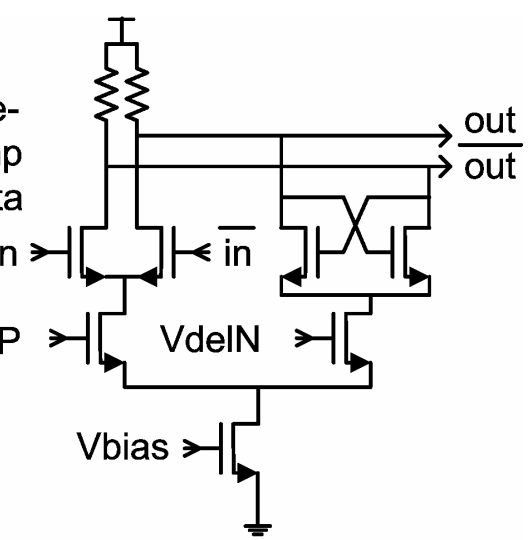

(b)

Fig. 17. (a) CML multiplexer. (b) CML delay tuning circuit.

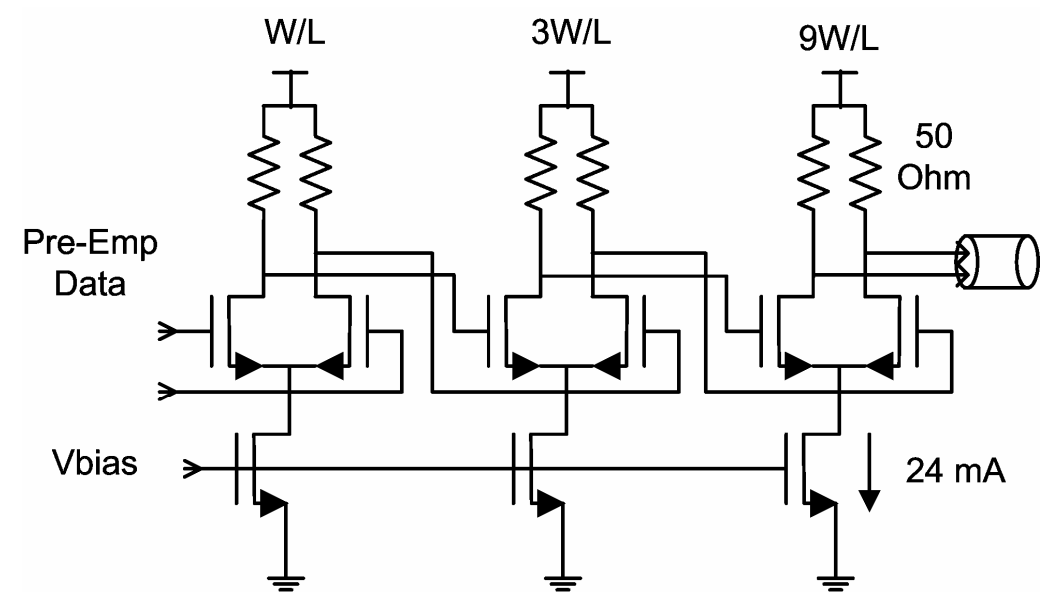

Fig. 18. Three-stage differential line driver of chip 1.

The line driver on chip 1 consists of three stages (Fig. 18). The final stage has a $50 \Omega$ on-chip termination resistance and a tail current of $24 \mathrm{~mA}$. This results in a nominal single-ended output swing of $600 \mathrm{mV}_{\mathrm{p}-\mathrm{p}}$ in a $50 \Omega$ cable. This corresponds to a differential voltage of $1.2 \mathrm{~V}_{\mathrm{p}-\mathrm{p}}$. The line driver power is $42 \mathrm{~mW}$ at $1.2 \mathrm{~V}$.

\subsubsection{Measurements}

A microphotograph of the first chip is shown in Fig. 19(a). This chip measures $1 \mathrm{x} 1 \mathrm{~mm}^{2}$, and has an active area of approximately $150 \times 150 \mu \mathrm{m}^{2}$. All chip I/Os have on-chip $50 \Omega$ termination and are ESD protected.

To evaluate the performance of the prototype chips, eye diagrams were created and BER tests run. Measurements were made using four cable assemblies and a PCB - all described and modeled in Chapter 2. For chip 1, the DC voltages/currents, including the power supply, were connected to a PCB by wirebonds, and the high speed inputs and outputs were probed. A $50 \Omega$ differential probe with 4 pins was used: ground-signal-signal-ground. If the high-speed data outputs were to be connected via a PCB, there might be an impedance mismatch between the PCB and the cable, causing reflections. (However, such reflections would be largely absorbed by the transmitter termination resistors. For the measurements we wanted to maximize signal integrity so we used probes.) The printed circuit board with the chip mounted is shown in Fig. 19(b). 


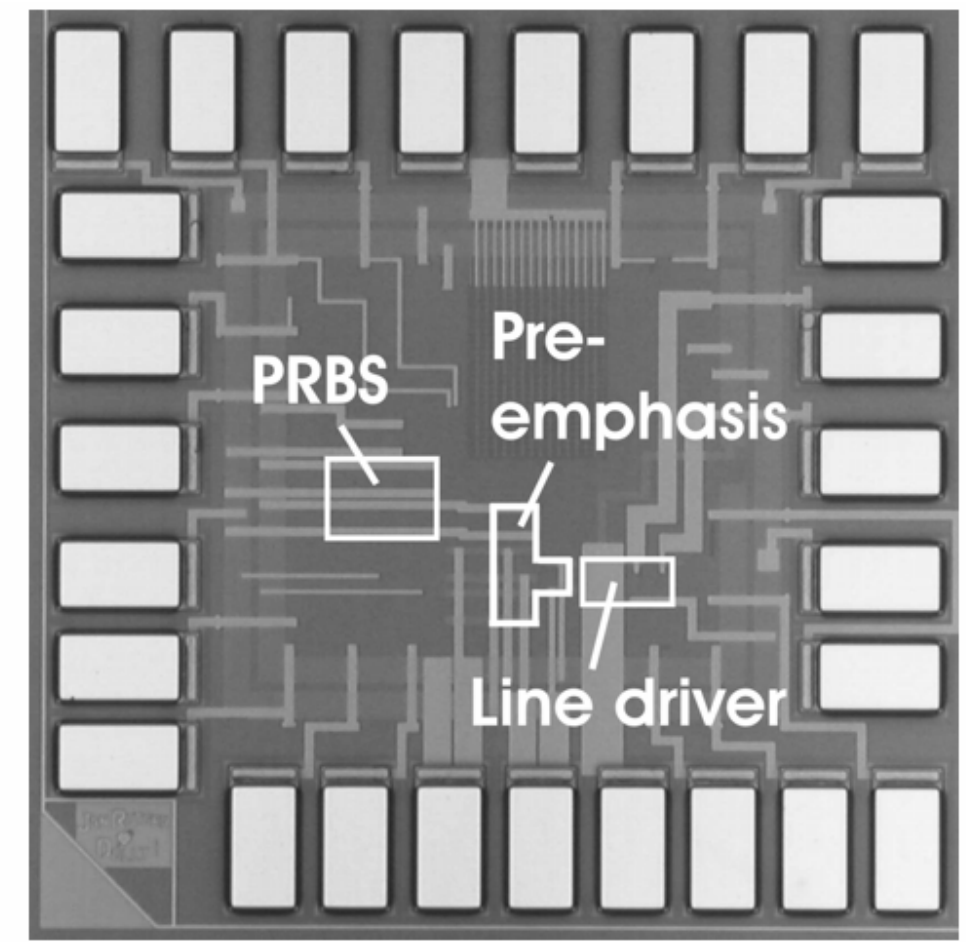

(a)

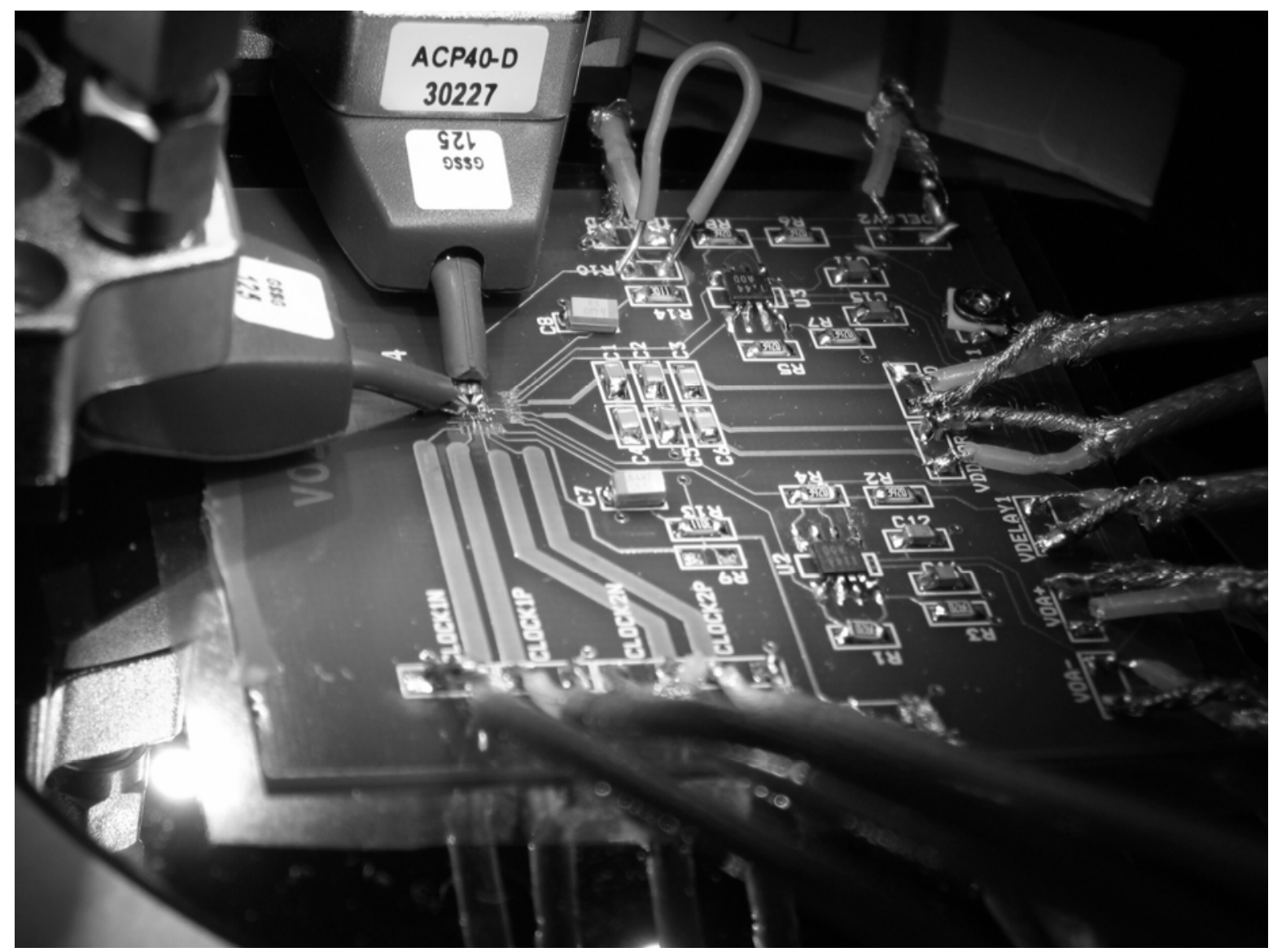

(b)

Fig 19. Chip micrograph, printed circuit board and probe configuration. (a). Chip micrograph, $1 \times 1 \mathrm{~mm} 2$. (b) Printed circuit board with chip wire-bonded to it, and measurement probes for high speed signals. 


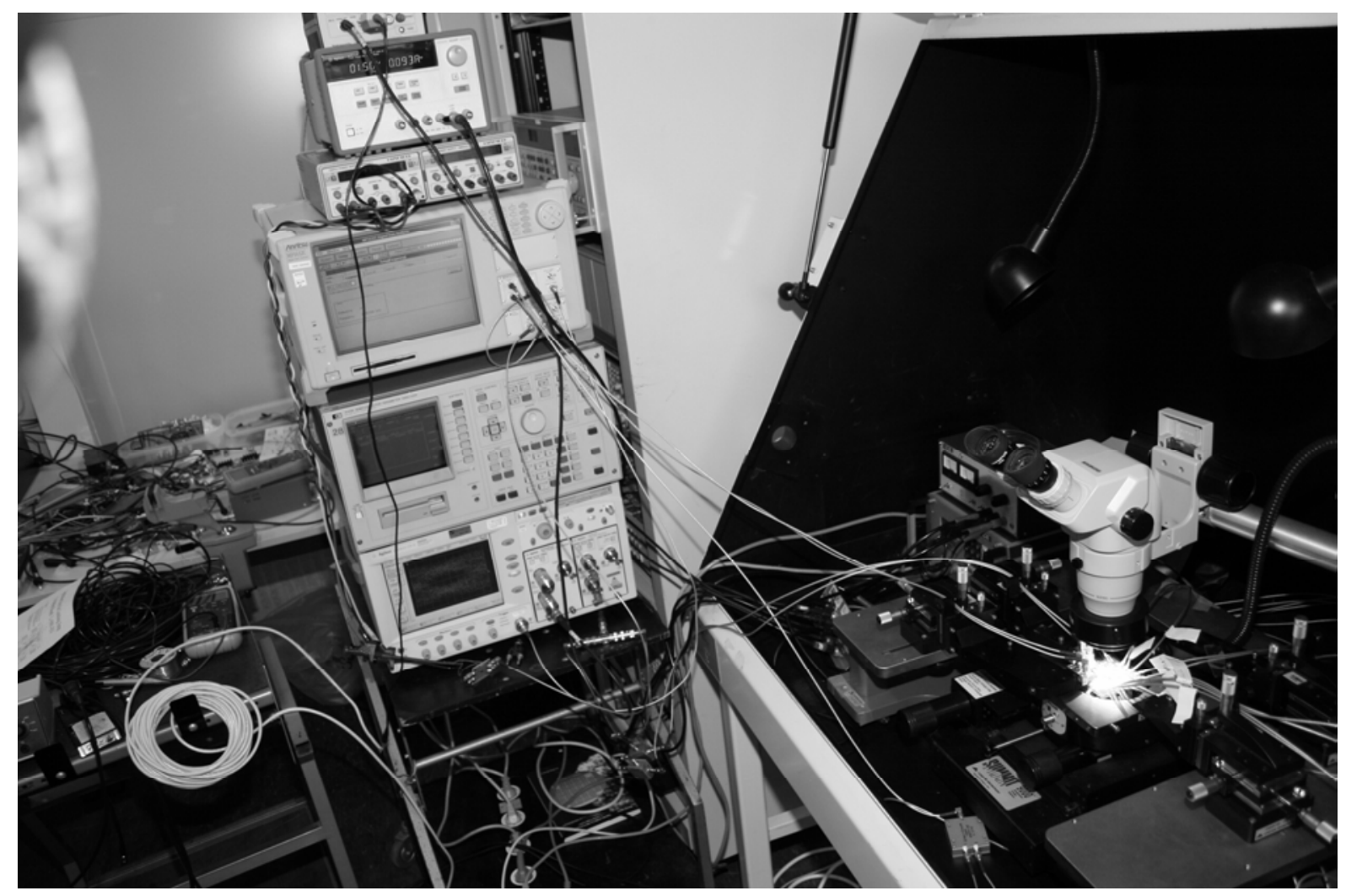

Fig 20. Measurement setup.

The measurement setup is shown in Fig. 20. The list of equipment used is as follows:

- Agilent DCA86100A Digital Communications Analyzer (oscilloscope, eye diagrams),

- Anritsu pattern generator / BER tester,

- HP semiconductor parameter analyzer (used for generating bias currents \& control voltages for setting the duty-cycle),

- several power supply units to give the required voltages,

- chip probe station.

\section{Effect of Adjustments in PWM Duty-Cycle}

In Figs. 21(a), (c) and (e), the effect of adjusting the PWM duty-cycle on the transmitter output can be seen. The left and right edges in the eye diagrams correspond to the symbol edges. In Figs. 21(b), (d) and (f), the responses of a 10-m RG-58CU cable to the preemphasized data stream with different pre-emphasis duty-cycles are shown. It can be seen that there is an optimum duty-cycle [Figs. 21(c) and (d)]. Under-emphasis is shown in Figs. 21(a) and (b) and over-emphasis in Figs. 21(e) and (f). Note that the time scale is the same in all the figures. The PWM pre-emphasis leaves the fastest data pattern ('101010') unchanged while it attenuates those data patterns with fewer transitions (e.g. a long string of $1 \mathrm{~s}$ ) .

\section{Eye Diagrams at Max. Loss Compensation}

The requirement for all measurements is to achieve a BER of $<10^{-12}$. The cable lengths are chosen such that this BER figure can be achieved at a bit rate of $5 \mathrm{~Gb} / \mathrm{s}$, the highest speed achievable using the TX circuitry. The channel loss at the Nyquist frequency can then be taken as a figure-of-merit for the equalizer. We call this the 'loss compensation' of the equalizer. In Figs. 22(a)-(d), measured eye diagrams of the cable outputs at $5 \mathrm{~Gb} / \mathrm{s}$ are shown. 
All four cable types, described in section 4.3, are shown. Figs. 22(a)-(c) are for the coaxial cables and Fig. 22(d) is for the differential cable. For the coaxial cables, one of the two transmitter outputs was used while the other was terminated with a $50 \Omega$ dummy load. For the differential cable, both transmitter outputs were used. The oscilloscope input is single-ended. Therefore, the eye-diagram shown in Fig. 22(d) was measured at the output of the differential limiting amplifier. For cables (a)-(c), the loss at $2.5 \mathrm{GHz}$ is $\sim 30 \mathrm{~dB}$. For cable (d), the loss at $2.5 \mathrm{GHz}$ is $19 \mathrm{~dB}$. (See also Chapter 2.) The total channel loss is a few $\mathrm{dB}$ more than the cable loss alone, because of additional losses from probes, short wire, bias tee and connectors. Using an external pattern generator and tester, the BER was tested with all cable assemblies at $5 \mathrm{~Gb} / \mathrm{s}$ and is $<10^{-12}$.

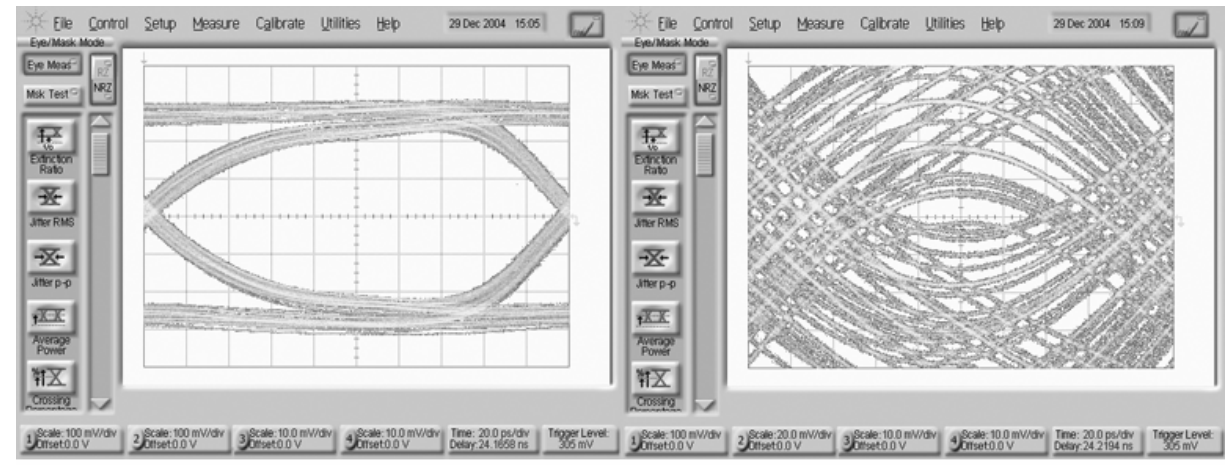

(a)

(b)

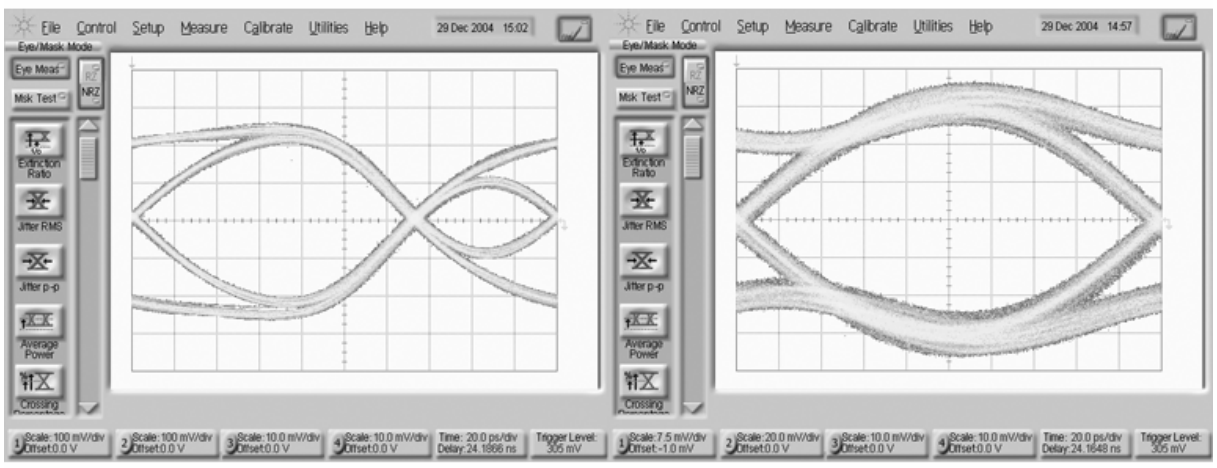

(c)

(d)

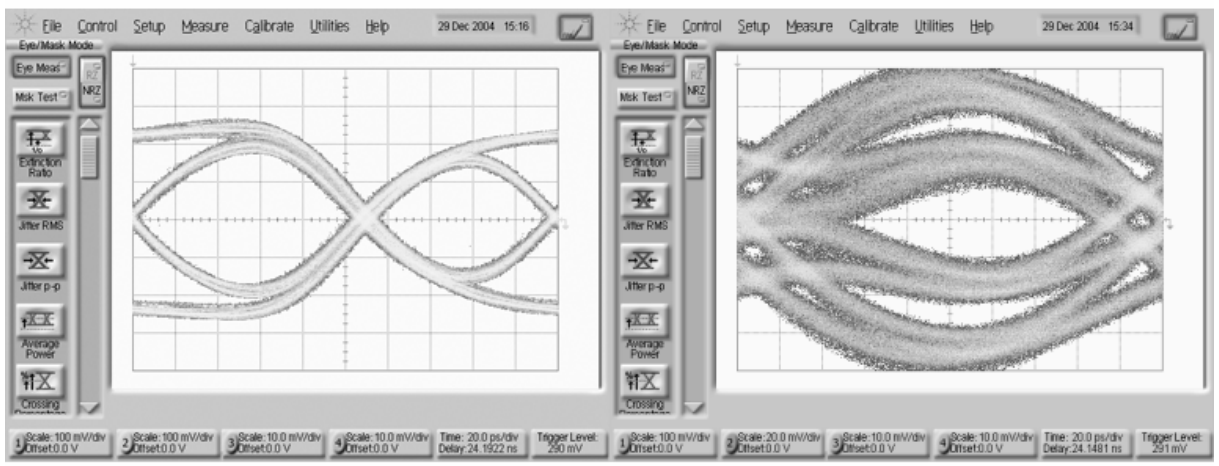

(e)

(f)

Fig. 21. Measured eye-diagrams for transmitter output and cable output (10 m RG-58CU) at $5 \mathrm{~Gb} / \mathrm{s}$. Horizontal axis $=20 \mathrm{ps} / \mathrm{div}$, vertical axis is $100 \mathrm{mV} / \mathrm{div}$. for (a), (c) and (e), and $20 \mathrm{mV} / \mathrm{div}$ for (b), (d) and (f).

(a) TX, no pre-emphasis (100\%). (b) RX, no pre-emphasis $(100 \%)$. (c) TX, optimum preemphasis (66\%). (d) RX, optimum pre-emphasis (66\%). (e) TX, strong pre-emphasis (55\%).

(f) RX, strong pre-emphasis (55\%). 


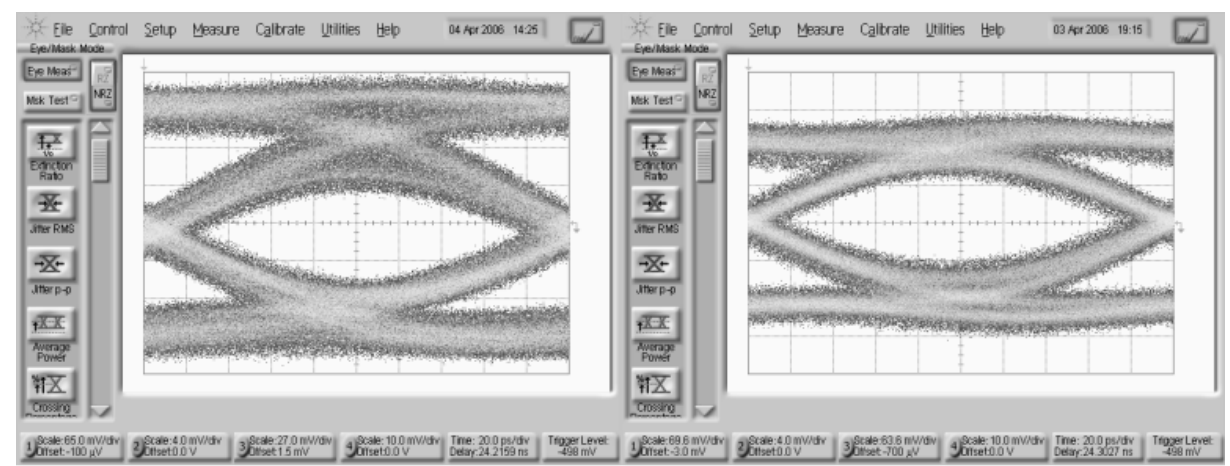

(a)

(b)

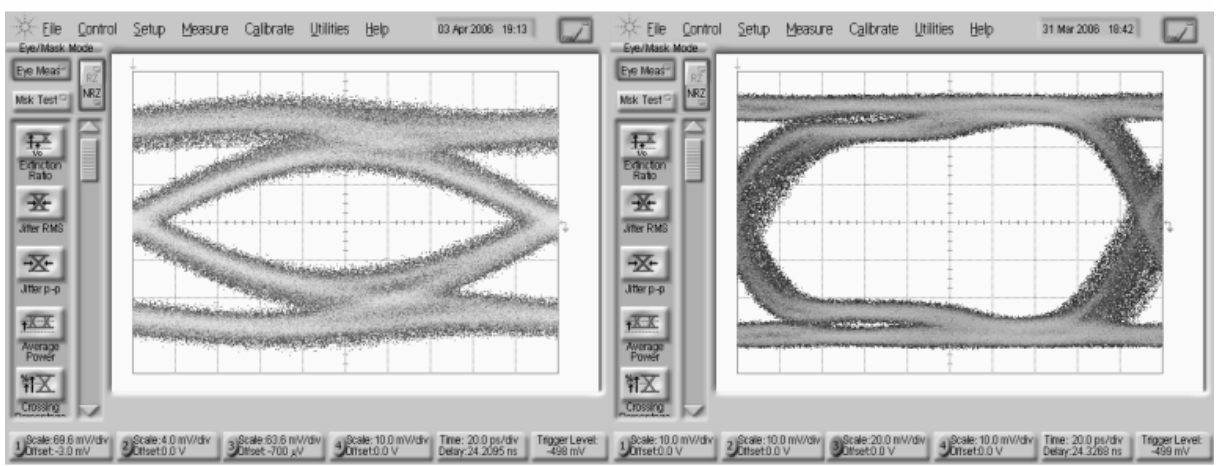

(c)

(d)

Fig. 22. Measured output eyes of chip 1 at $5 \mathrm{~Gb} / \mathrm{s}$ and BER $<1 \cdot 10^{-12}$. Horizontal axis $=$ $20 \mathrm{ps} / \mathrm{div}$, vertical axis is $4 \mathrm{mV} / \mathrm{div}$. for (a)-(c) and $20 \mathrm{mV} / \mathrm{div}$. for (d).

(a) $25 \mathrm{~m}$ RG-58CU. (b) $130 \mathrm{~m}$ Aircom+. (c) $80 \mathrm{~m}$ Aircell7. (d) $15 \mathrm{~m}$ 10GBASE-CX4 24AWG after differential limiting amplifier.

At a channel loss of $>30 \mathrm{~dB}$, the small cable output amplitude imposes a high demand on receiver sensitivity. For the BER measurements, a limiting amplifier was used with a $1 \mathrm{mV}$ input offset. Using the fully differential transmitter capabilities with cable (d) offers the advantage of a $6 \mathrm{~dB}$ higher (differential) swing while also rejecting common mode noise.

In Table 1, a comparison with other published work is given. For the 2PAM systems, none of the pre-emphasis filters that use 2 taps offer more than $18 \mathrm{~dB}$ loss compensation [Lee], [Kudoh]. A 5-tap FIR filter reached $30 \mathrm{~dB}$ in a 2 PAM system, but only at $3.125 \mathrm{~Gb} / \mathrm{s}$ [Gai]. More taps can offer higher loss compensation but at the expense of increasing complexity, possibly causing accuracy and speed problems. Furthermore, algorithm convergence for automatically finding the optimum equalizer coefficients is more troublesome than for an equalizer with only one 'knob'. The PWM-PE filter presented here offers a record loss compensation $(33 \mathrm{~dB})$, at a bit rate of $5 \mathrm{~Gb} / \mathrm{s}$.

(In [Farjad-Rad] a 4PAM transmitter is described, which cannot be compared directly because it theoretically requires a $9.5 \mathrm{~dB}$ higher SNR for the same BER, due to the smaller eye opening.)

In Table 2, the electrical characteristics of the transmitter are given. Power dissipation figures are hard to compare because most publications only give total figures. In the current proof-ofconcept design, the clock buffering takes much of the power budget, but this drain can be reduced when internal clocks are available on the IC. Because of the simplicity of the preemphasis method, both the chip area and power consumption can be very low. 


\begin{tabular}{|l|l|l|l|l|}
\hline Ref. & $\mathrm{R}$ & Loss & Process & Type \\
\hline [Farjad-Rad] & $8 \mathrm{~Gb} / \mathrm{s}$ & $\sim 10 \mathrm{~dB}$ & $0.3 \mu \mathrm{m}$ & 2-tap FIR \\
\hline [Lee] & $4 \mathrm{~Gb} / \mathrm{s}$ & $\sim 10 \mathrm{~dB}$ & $0.25 \mu \mathrm{m}$ & 2-tap FIR \\
\hline [Kudoh] TX only & $5 \mathrm{~Gb} / \mathrm{s}$ & $18 \mathrm{~dB}$ & $0.13 \mu \mathrm{m}$ & 2-tap FIR \\
\hline [Gai] TX only & $3.125 \mathrm{~Gb} / \mathrm{s}$ & $30 \mathrm{~dB}$ & $0.11 \mu \mathrm{m}$ & 5-tap FIR \\
\hline this work & $5 \mathrm{~Gb} / \mathrm{s}$ & $33 \mathrm{~dB}$ & $0.13 \mu \mathrm{m}$ & PWM \\
\hline
\end{tabular}

Table 1. Pre-emphasis comparison with other work. In [Kudoh] and [Gai] the loss compensation from the TX pre-emphasis was measured by turning off the receiver equalizer.

\begin{tabular}{|l|l|}
\hline Bit rate (2-PAM) & $5 \mathrm{~Gb} / \mathrm{s}$ \\
\hline U-I & $200 \mathrm{ps}$ \\
\hline TX amp. ( $\mathbf{V}_{\text {p-p }}$ ) nom. & $1.2 \mathrm{~V}($ dif), \\
& $600 \mathrm{mV}$ (s-ended) \\
\hline Channel loss @ 2.5GHz & $33 \mathrm{~dB}$ \\
\hline $\mathbf{V}_{\text {sup }}$ & $1.2 \mathrm{~V}$ \\
\hline Power (pre-emphasis) & $12 \mathrm{~mW}$ \\
\hline Power (line driver) & $42 \mathrm{~mW}$ \\
\hline Power (clock buffering) & $39 \mathrm{~mW}$ \\
\hline Power (on-chip PRBS) & $17 \mathrm{~mW}$ \\
\hline
\end{tabular}

Table 2. Electrical characteristics of transmitter.

\subsubsection{Chip 2: Design and Measurements}

The high cable loss results in a low amplitude at the cable output, which can lead to problems at the receiver side. The SNR might be too low, or the received signal amplitude might be below the receiver offset. A usual value for the receiver offset is $\sim 50 \mathrm{mV}$. Therefore, it would be convenient to have a transmitter with a higher output swing. With this in mind, a new line driver was designed, which is described in 4.5.2.1. The duty-cycle tuning circuit that was used is described in 4.5.2.2. Finally, the measurement results are given in 4.5.2.3.

\subsubsection{Line Driver}

The line driver circuit of chip 2 extends the line driver of chip 1 by cascading a cascode stage. High-voltage transistors are not needed. This enables a maximum output voltage of $2.5 \mathrm{~V}$. The output current is adjustable from 36 to $74 \mathrm{~mA}$, resulting in a $1.8 \mathrm{Vp}$-p single-ended voltage swing in $50 \Omega$ or $25 \Omega$, the latter in the case of an external TX termination resistor. Compared to the first chip, the TX power is almost $10 \mathrm{~dB}$ higher. This would lead to high power dissipation in an on-chip TX termination resistor, dissipating $(1.8)^{2} / 50=65 \mathrm{~mW}$. The excess heat could pose a problem when many high-speed links are operated in parallel. For that reason, the TX termination resistor was left out and the driver is open-drain. It is shown in Fig. 23. The cable needs to be terminated to $2.5 \mathrm{~V}$, in order to supply the driver bias current. 


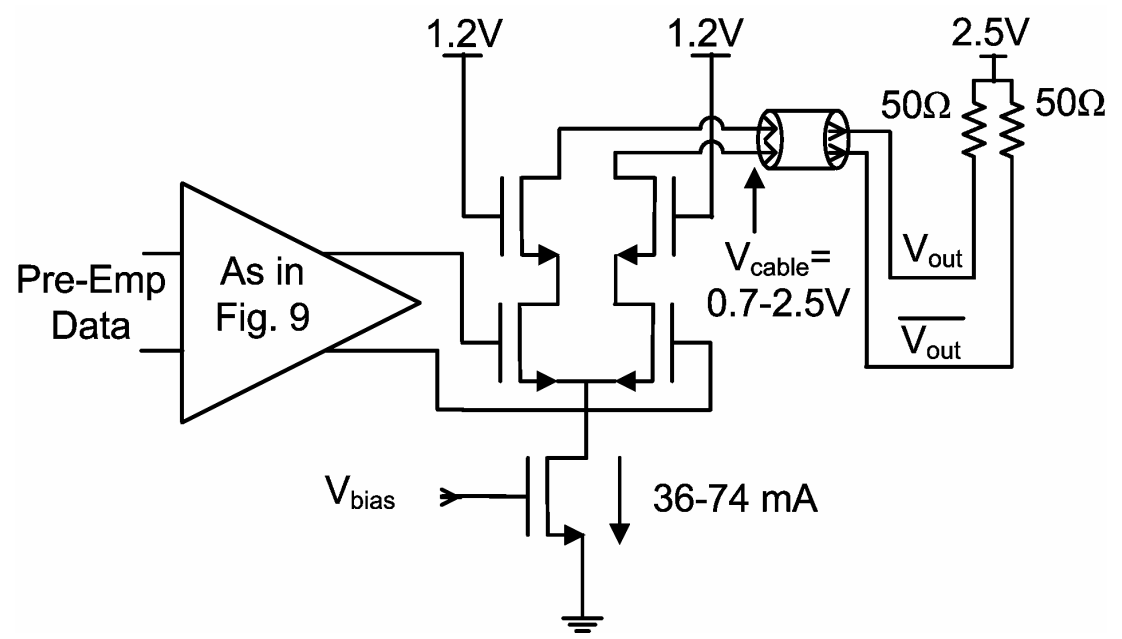

Fig. 23. Line driver of chip 2 with cable and termination.

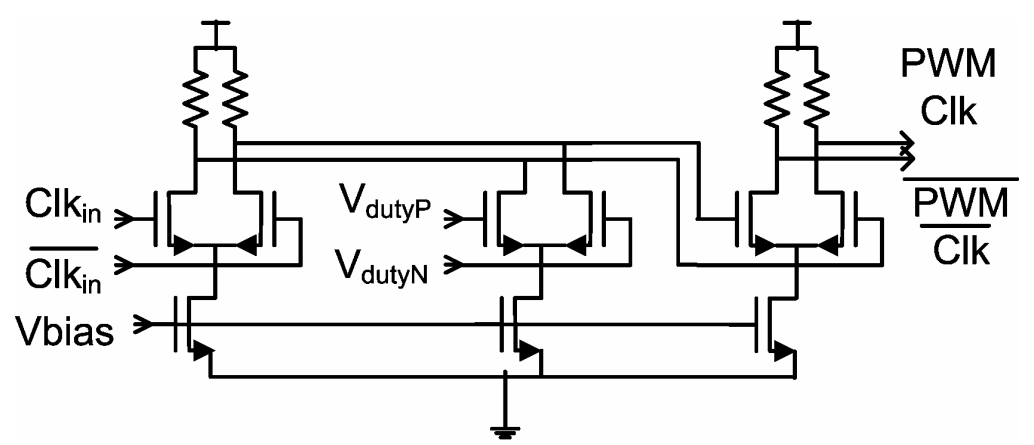

Fig. 24. Duty-cycle tuning circuit of chip 2.

\subsubsection{Duty-cycle tuning circuit}

A duty-cycle tuning circuit different from that used in chip 1 is chosen, because the latter has some drawbacks. It requires the adjustment of two voltages, and its duty-cycle is not nominally set at $50 \%$, which would be convenient for a cable with significant loss. The circuit on chip 2 does have a nominal setting of $50 \%$ [Westergaard]. It outputs a duty-cycle of $50 \%$ at a differential voltage $V_{d u t y P}-V_{d u t y N}=0 \mathrm{~V}$, as shown in Fig. 24. The differential voltage $V_{\text {duty }}-V_{\text {duty } N}$ controls the the offset of the middle differential pair. The $\mathrm{dV} / \mathrm{dt}$ voltage slope then converts this into a duty-cycle change.

\subsubsection{Measurements}

A micrograph of chip 2 is shown in Fig. 25. The chip measures $1 \times 0.9 \mathrm{~mm}^{2}$, and has an active area of approximately $100 \times 100 \mu \mathrm{m}^{2}$. The control voltages, bias currents, power supply, and the high speed in-/outputs were all connected via probes. Because our oscilloscope is terminated to ground, a bias tee was used at the cable output. In this way, we could still supply the driver bias current while terminating the cable to ground. The DC input of the bias tee was connected to $1.65 \mathrm{~V}$, which is the threshold voltage of the driver output for $1.8 \mathrm{~V}$ swing $(2.5-1.8 / 2=1.65 \mathrm{~V})$.

The output of the TX at $4 \mathrm{~Gb} / \mathrm{s}$ is shown in Fig. 26, clearly showing its high swing. In Figs. 27(a)-(d), measured eye diagrams of the cable outputs are shown. The maximum speed achieved with cables (a)-(c) is $3 \mathrm{~Gb} / \mathrm{s}$ for a BER $<1 \cdot 10^{-12}$. The loss of cables (a)-(c) at $1.25 \mathrm{GHz}$ is $\sim 20 \mathrm{~dB}$. For the differential cable (d), a speed of $5 \mathrm{~Gb} / \mathrm{s}$ was achieved. The loss of 
this cable at $2.5 \mathrm{GHz}$ is $19 \mathrm{~dB}$. A possible explanation for the slightly lower performance than chip $1(30 \mathrm{~dB})$ is the data-dependent noise at the TX output. In Fig. 26, separate lines can be identified. (The $2^{7}-1$ PRBS pattern repeats after 127 bits. The lines disappear when the PRBS pattern is switched from $2^{7}-1$ to $2^{31}-1$.) The data-dependent noise is probably caused by the lack of an on-chip termination resistance. For canceling these reflections, the use of a $50 \Omega$ onchip TX termination is commonly mentioned in the literature. To save power, we removed this termination resistor but, as stated earlier, this might have caused the decreased performance of chip 2. Ideally we would like to save power and cancel reflections at the same time, but for this we would need to find (for example) a suitable virtual impedance driver concept.

The measured eye openings in Figs. 27(a)-(c) are clearly larger than for chip 1, due to the larger TX output swing, even when the $8 \mathrm{~dB}$ difference in channel loss at the (lower) Nyquist frequency is taken into account.

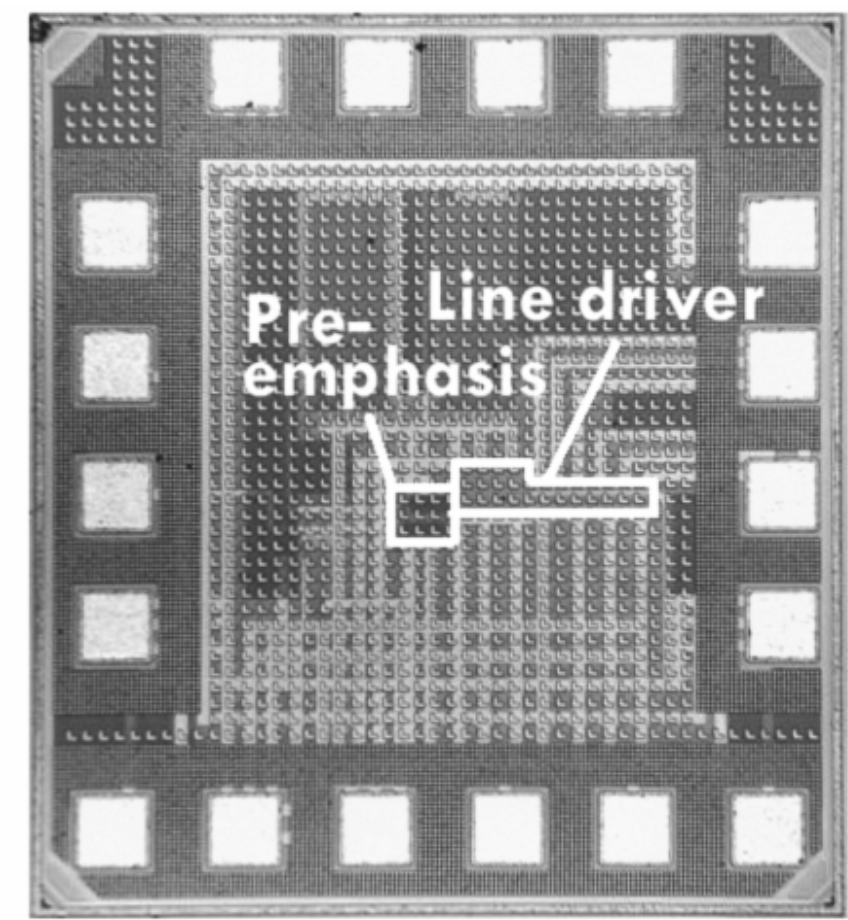

Fig. 25. Chip 2 micrograph, 1x0.9 mm2.

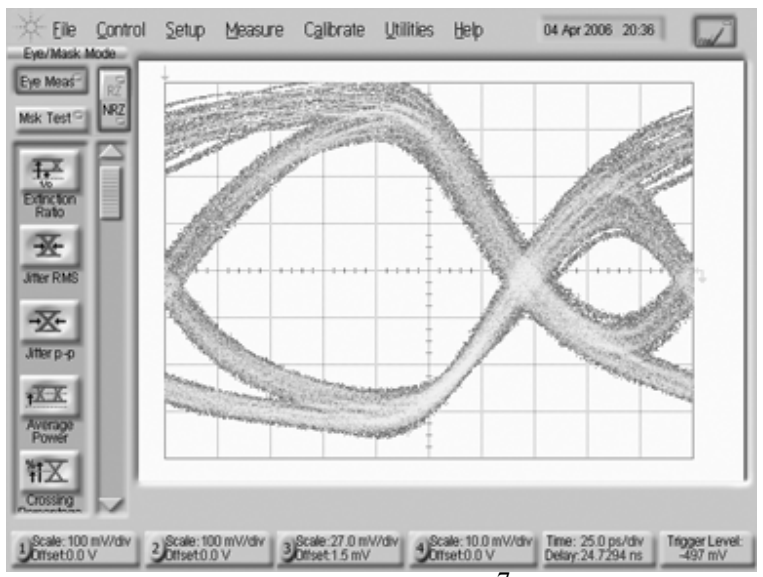

Fig. 26. Single-ended transmitter eye of chip 2, for $2^{7}-1$ PRBS pattern at $4 \mathrm{~Gb} / \mathrm{s}$. Horizontal axis $=25 \mathrm{ps} /$ div., Vertical axis $=100 \mathrm{mV} / \mathrm{div}$. (10dB att. $)$ 


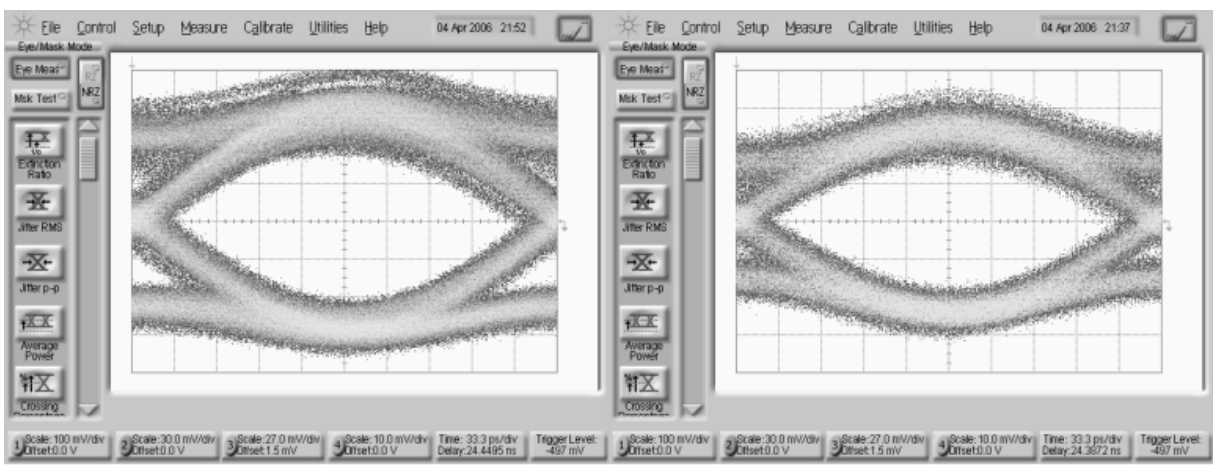

(a)

(b)

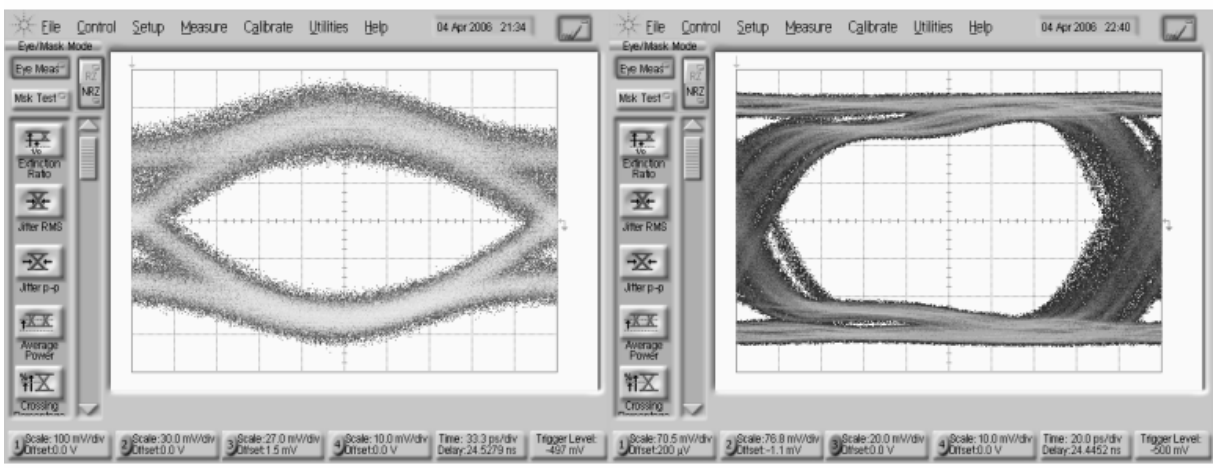

(c)

(d)

Fig. 27. Measured output eyes of chip 2 at BER $<1 \cdot 10^{-12}$. Horizontal axis is $33.3 \mathrm{ps} / \mathrm{div}$ for (a)-

(c), and 20ps/div. for (d). Vertical axis is $30 \mathrm{mV} / \mathrm{div}$. for (a)-(c), and $20 \mathrm{mV} / \mathrm{div}$. for (d).

a) $25 \mathrm{~m} \mathrm{RG}-58 \mathrm{CU}$ at $3 \mathrm{~Gb} / \mathrm{s}$. (b) $130 \mathrm{~m}$ Aircom+ at $3 \mathrm{~Gb} / \mathrm{s}$. (c) $80 \mathrm{~m}$ Aircell 7 at $3 \mathrm{~Gb} / \mathrm{s}$.

(d) $15 \mathrm{~m}$ 10GBASE-CX4 24AWG at $5 \mathrm{~Gb} / \mathrm{s}$ after differential limiting amplifier.

\subsubsection{Chip 3: measurements on a PCB trace}

In data-communication over legacy FR4 backplanes (printed circuit boards), the most important speed limit is not the skin effect, as in cables, but dielectric polarization and relaxation. A 2-5 tap symbol-spaced FIR filter is commonly used for TX pre-emphasis (PE) on backplanes [Stonick], [Balan], [Payne], [Krishna], [Zerbe]. We have shown above that pulse-width modulation (PWM) TX PE can effectively replace FIR TX PE for copper cables. We show here that it also works for printed circuit boards. In subsection 4.5.3.1 the circuit of a $0.13 \mu \mathrm{m}$ chip, that was designed for this purpose, is described. In 4.5.3.2, the measurement results are given.

\subsubsection{Circuit description}

A test transmitter chip was designed in $0.13 \mu \mathrm{m}$ CMOS. At the time of the design of the chip, we thought that the impulse response from dielectric loss was more symmetrical than it is in reality (see also the footnote on page 79), so an extra degree of freedom was added. Where previously the pulse was always on the left side, we can now place it at an arbitrary position, e.g. in the middle. This is illustrated in Fig. 28(b), along with the block diagram and electrical signals in Fig. 28(a). The circuit was designed in differential CML. The duty-cycle control is different from the other chips and is based on a divider and XOR circuit [Nam]. The delays between signal ' $I$ ' and 1 and signal ' $Q$ ' and 2, delay1 and delay2 respectively, are generated with four cascaded tunable delay sections, shown in Fig. 17(b). The differential line driver, illustrated in Fig. 18, outputs a single-ended swing of $600 \mathrm{mVp}-\mathrm{p}$ in $50 \Omega$ (differential 1.2Vp-p in $100 \Omega)$. 


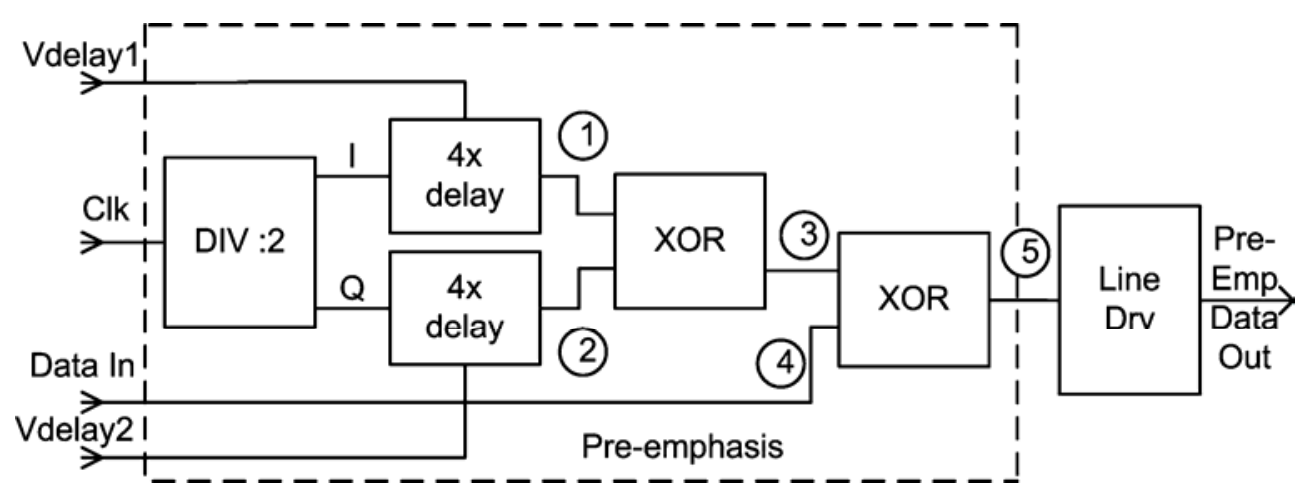

(a)

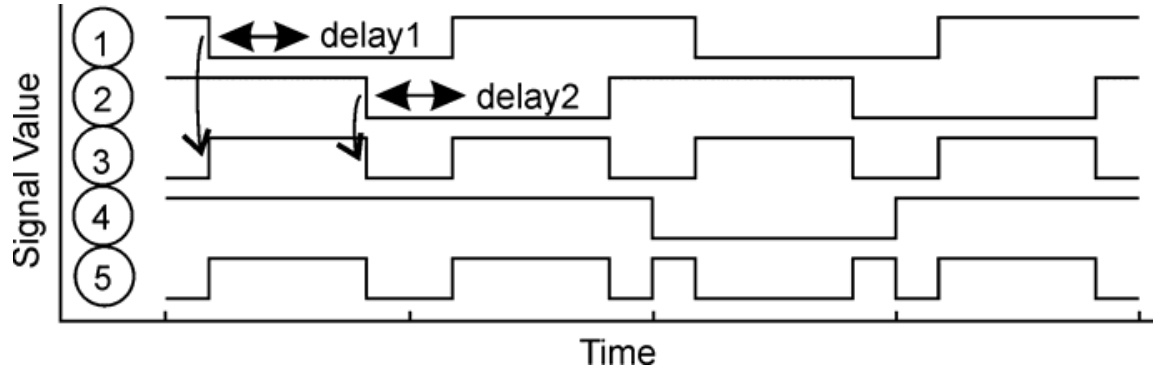

(b)

Fig. 28. Chip block diagram and signals. (a) Block diagram. (b) Signals.

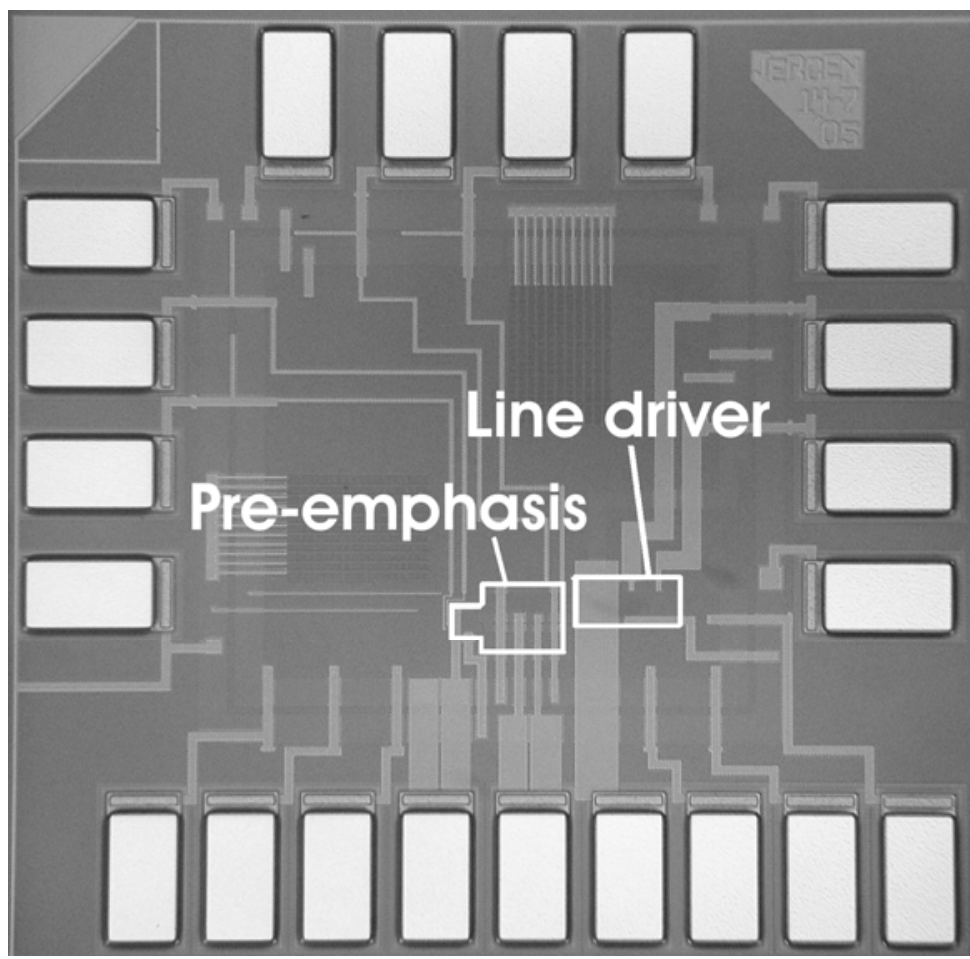

Fig. 29. Chip micrograph. Line driver width is $110 \mu \mathrm{m}$. 


\subsubsection{Measurements}

In Fig. 29 the chip microphotograph is shown. The measurement goal was again to evaluate the maximum loss compensation capability of the PWM-PE technique. A very long PCB trace of $270 \mathrm{~cm}$ (106") of FR4 was used to be able to achieve a high channel loss at the Nyquist frequency. (See also Chapter 2 for a description and photograph.) The trace is single-ended. It is a microstrip with a characteristic impedance of $50 \Omega$. There are no vias, and at the end there are SMA connectors. The total channel (PCB $+1.75 \mathrm{~m}$ coaxial cable + bias tee) has $25 \mathrm{~dB}$ loss at the Nyquist frequency of $2.5 \mathrm{GHz}$. Measurements were made using chip probes. One of the two TX outputs was connected to the trace, and the free TX output was directly connected to the $50 \Omega$ oscilloscope.

In Fig. 30, the transmitter output obtained using the extra degree of freedom (the pulse position) is shown. Two bits are shown in the picture: the length of the x-axis is two bit times. Looking at the left half of the figure (the first bit), during one bit time, if a ' 1 ' bit is transmitted, the voltage first goes negative, then positive, and then negative again. Comparing the two bits, we can see some asymmetry. The XOR implementation probably causes this. Looking at Fig. 28(b), we can see that, when the duty-cycle of the (half-frequency) signals 1 and 2 is not exactly $50 \%$, signal 3 is different for even bits than for odd bits.

In Fig. 31, the eye diagrams from these measurements are shown, both without PE and for the optimum PE setting. A bit rate of $5 \mathrm{~Gb} / \mathrm{s}$ was achieved at a BER of $1 \cdot 10^{-12}$ over $270 \mathrm{~cm}$ of FR4 ( $2^{7}-1$ PRBS pattern). The $25 \mathrm{~dB}$ loss compensation at the Nyquist frequency represents a good performance, compared to the $\sim 20 \mathrm{~dB}$ achieved in [Balan], [Payne], [Krishna]. The total power dissipation of the test chip is $56 \mathrm{~mW}$, of which the line driver consumes $42 \mathrm{~mW}$, and the PE circuit itself dissipates $14 \mathrm{~mW}$.

It should be taken into account that channel conditions are different than in the mentioned literature; in the mentioned literature the channels had impedance discontinuities, for example from vias. However, our figure was achieved without any RX equalization (described in the literature as used to cancel reflections), using only TX pre-emphasis. Reflections from impedance discontinuities can be compensated for by adding other, more conventional techniques, especially a decision feedback equalizer (DFE) at the RX side, as is done in [Balan], [Payne], and [Krishna].

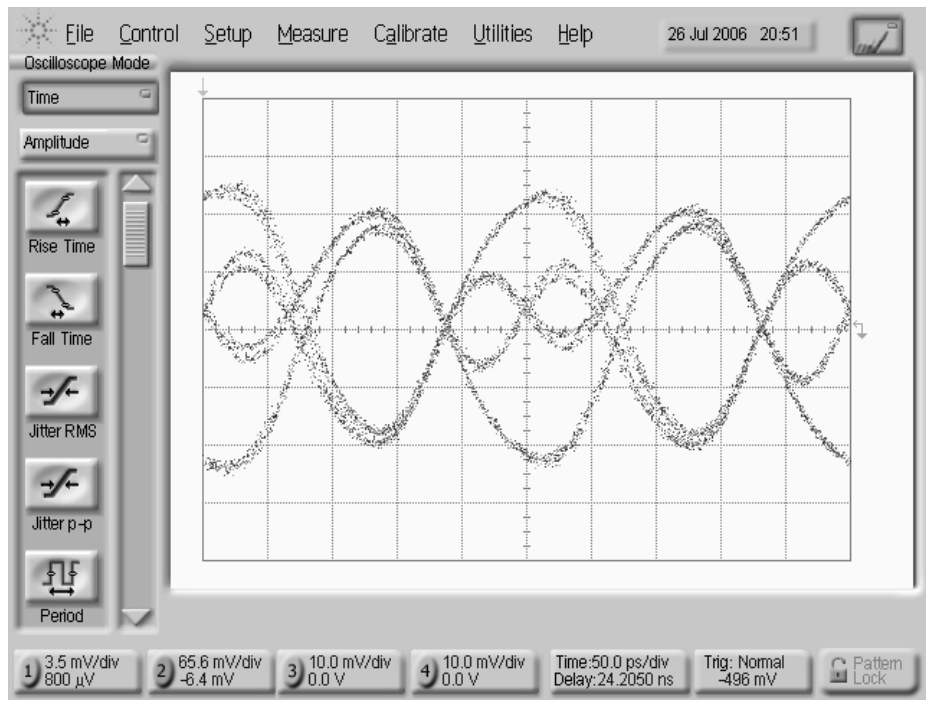

Fig. 30. Output of chip 3 (two bits), showing the extra degree of freedom: the pulse position. 


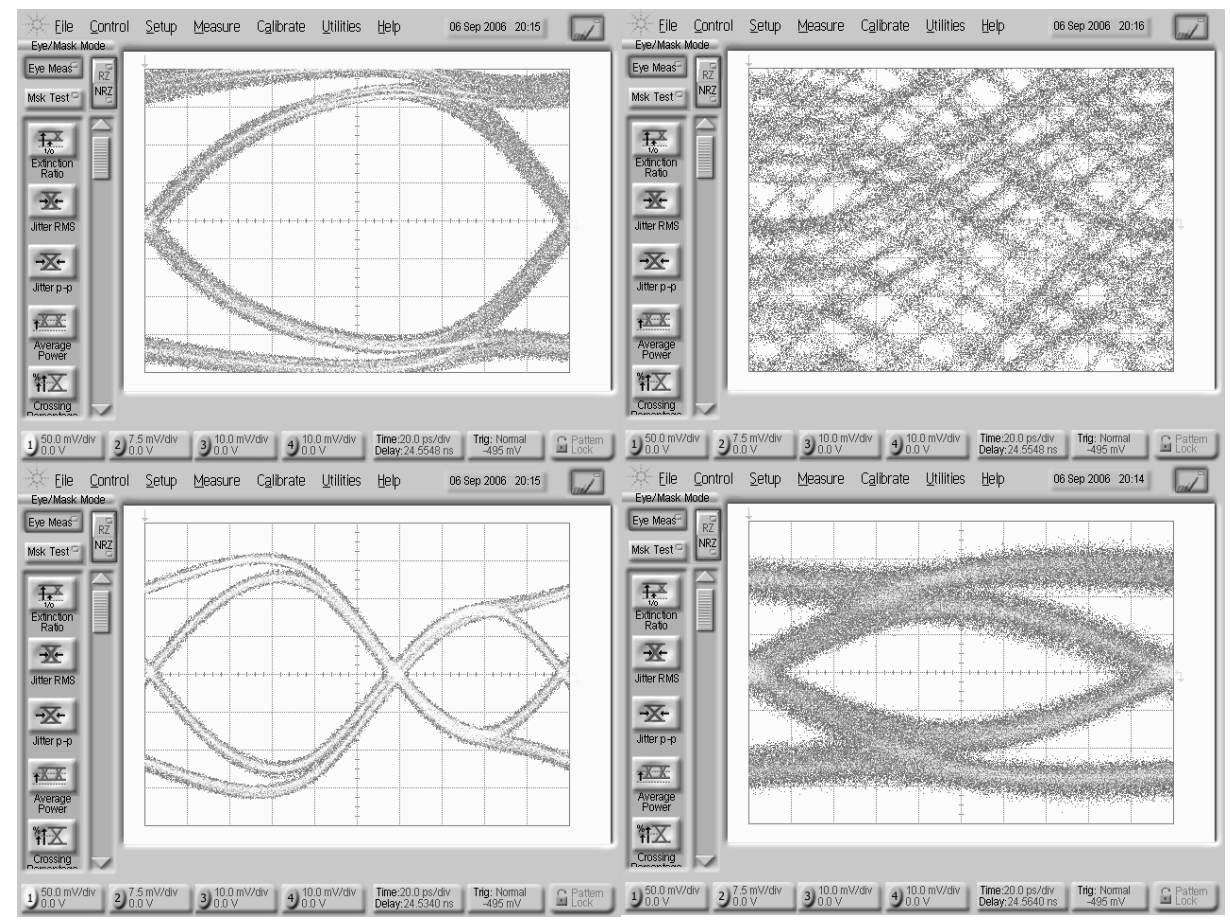

Fig. 31: $5 \mathrm{~Gb} / \mathrm{s}$ over $270 \mathrm{~cm}$ (106") FR4, channel loss at Nyquist frequency is $25 \mathrm{~dB}$. Top left: input to pcb with no pre-emphasis, right: output from pcb without pre-emphasis. Bottom left: input to pcb with optimum pre-emphasis, right: output from pcb with optimum pre-emphasis.

Note that the $\mathrm{x}$-axis length is one bit time. $Y$-axis, left: $50 \mathrm{mV} / \mathrm{div}$, right: $7.5 \mathrm{mV} / \mathrm{div}$.

The additional degree of freedom that enables us to place the pulse at an arbitrary position in the bit turned out not to be necessary to compensate for the dielectric loss. The notion that a precursor filter tap is not necessary for PCBs is confirmed in [Ren], where analysis shows that the precursor tap often used for pre-emphasis for PCBs is in fact only necessary because the clock-and-data recovery (CDR) chooses a non-optimum sampling point. A better sampling point can be found, without the use of a precursor tap.

\subsection{Conclusions}

High-speed data links over copper channels can be effectively equalized using pulse-width modulation (PWM) pre-emphasis. This provides an alternative to the usual 2-tap symbolspaced FIR. The use of PWM pre-emphasis allows a channel loss at the Nyquist frequency of $\sim 30 \mathrm{~dB}$, compared to $\sim 20 \mathrm{~dB}$ for a 2-tap symbol-spaced FIR filter. The PWM method does not tune the pulse amplitude as for FIR pre-emphasis, but instead exploits timing resolution. This fits neatly with CMOS technology trends toward higher switching speeds and lower voltage headroom.

The PWM duty-cycle determines the shape of the transfer function. Therefore, the filter has only one adjustment parameter. This makes convergence of an algorithm for automatic adaptation straightforward. Spectral analysis illustrates that, compared to a 2-tap FIR filter, the PWM filter transfer function fits better to the copper channel. The equalized channel transfer function is the flattest for PWM pre-emphasis, compared to both half-symbol-spaced and symbol-spaced 2-tap FIR filters. At the Nyquist frequency, the calculated magnitude of 
the PWM filter transfer function has a value of 1 while its derivative is non-zero, unlike with the 2-tap SSF filter. The PWM filter transfer function can be seen as a higher order filter with only one adjustment parameter. Time domain simulations with a skin-effect-only channel show that approximately twice the bit rate can be achieved compared to a 2-tap SSF for the same peak distortion.

Three transmitter chips were designed, manufactured and tested as a proof-of-concept. The higher the loss compensation demanded, the lower the allowable jitter in the duty-cycle. The pre-emphasis technique can be implemented on only a small chip area and with low power consumption. Chip 2 has a higher TX swing than chip 1, to compensate for the high channel loss. The TX termination resistance was left out of chip 2 to keep power dissipation low, however it suffers from some data-dependent noise at the TX output. The chips were tested with three types of coaxial cable and one differential cable. The cable lengths were $25 \mathrm{~m}$, $80 \mathrm{~m}, 130 \mathrm{~m}$ and $15 \mathrm{~m}$, respectively. The loss at $2.5 \mathrm{GHz}$ is $\sim 30 \mathrm{~dB}$ for the three coaxial cables and $19 \mathrm{~dB}$ for the differential cable. A BER $<10^{-12}$ at $5 \mathrm{~Gb} / \mathrm{s}(2-\mathrm{PAM})$ was achieved with chip 1 for all cable assemblies. Transmission of a 2-PAM $5 \mathrm{~Gb} / \mathrm{s}$ data signal over $25 \mathrm{~m}$ of low-cost, low-end, standard RG-58U coaxial cable was demonstrated with a BER $<10^{-12}$. This corresponds to a record loss compensation of more than $30 \mathrm{~dB}$ at the Nyquist frequency of $2.5 \mathrm{GHz}$. On a PCB, a loss compensation of $25 \mathrm{~dB}$ was achieved using chip 3. 


\section{Chapter 5}

\section{Multitap PWM pre-emphasis}

\subsection{Introduction}

In Chapter 4, it was shown that the simple PWM pre-emphasis filter fits very well to cables and PCBs, and can provide up to $30 \mathrm{~dB}$ of loss compensation. An interesting question is whether the pulse-width filtering technique can be extended to equalize channels with a more complex transfer function. As is well known, with a FIR filter, this is done by increasing the number of taps. Using multitap FIR pre-emphasis, more poles and zeros can be added to the filter transfer function. We can then for example make band pass filters, increase the filter order, and cancel reflections, e.g. on backplane PCBs [Stonick].

The question that we address in this chapter is: would the same be possible for the PWM filter? The goal is to make a transmitter that, unlike a FIR pre-emphasis filter, still switches between only two discrete output voltages, while still offering the degrees of freedom of a multitap FIR filter. This would enable a switching transmitter architecture, with possible advantages in the light of CMOS scaling.

The key question now becomes: can we find a useful function to calculate the pulse-widths? These should be a function of multiple bits, as in the FIR filter, where the amplitudes are a function of multiple bits. If we are able to do so, which extra degrees of freedom do we gain? Are these extra degrees of freedom orthogonal? In this chapter, we explore the functionality that these multitap PWM filters can offer and we analyze their power spectral density (PSD) functions.

The remainder of this chapter is divided into four sections as follows. Section 5.2 starts by introducing the principle of the multitap PWM filter. We first convert the output of a multitap symbol-spaced FIR (SSF) filter into a PWM output by using the 'same-area approach'. We show with transient simulations that, for an example channel, using such a multitap PWM transmit filter results in nearly identical eye diagrams at the receive side as for a multitap SSF transmit filter. Next, Section 5.3 introduces a method to calculate the autocorrelation function and the PSD of such a multitap PWM filter. Next, in section 5.4, an optimization of the PWM function is made that lowers the number of transitions per second, decreasing the width of the PSD. Finally, conclusions are drawn in section 5.5.

Throughout the sections, the example of a three-tap PWM filter is used. However, the calculation methods can be extended to more than three taps. 


\subsection{Principle of the multitap PWM filter}

In this section, we develop the concept of a multitap PWM pre-emphasis filter. As a start, in subsection 5.2.1, we try to convert the output of a multitap SSF filter into a PWM output by making sure that the area under the transmitted waveforms is equal. This is first done using a PWM pre-emphasis transmitter with three output voltage levels $(-1 \mathrm{~V}, 0 \mathrm{~V}$ and $1 \mathrm{~V})$. The second step (in subsection 5.2.2) is a PWM filter with two output voltage levels (-1V and 1V), which would be more straightforward to implement. Transient simulations and eye diagrams are shown to demonstrate the feasibility of the multitap PWM filter. This section follows an intuitive approach; mathematical models are developed later in this chapter.

\subsubsection{Same-area approach to translate FIR filter to PWM filter}

A first step can be taken as follows. The basic idea for the multitap PWM filter is that the pulse-width is a function of multiple bits, in the same way that the amplitude of a SSF filter is also a function of multiple bits. The question then arises: what is the correct function for this pulse width as a function of multiple bits? We replace the pulse amplitude modulated signal coming out of a SSF filter (which has variable amplitude and a fixed switching interval), with a pulse width modulated (PWM) signal that has a fixed amplitude and a variable switching interval. Our key assumption in making the translation is that if the area under both is the same, in terms of (absolute) voltage integrated over time, the channel response will also be the same. This is a general assumption behind many well-known PWM schemes [Nielsen].

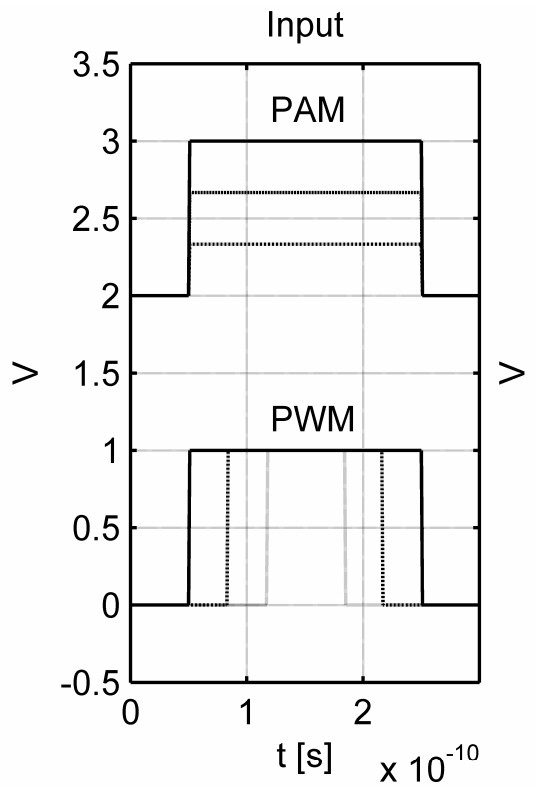

(a)

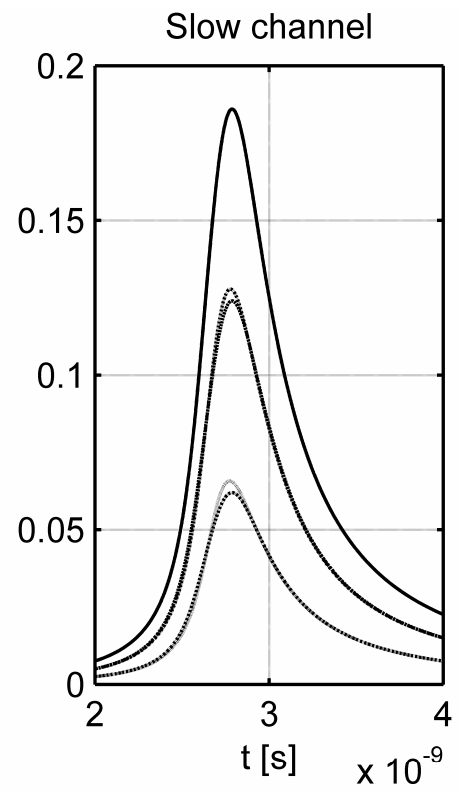

(b)

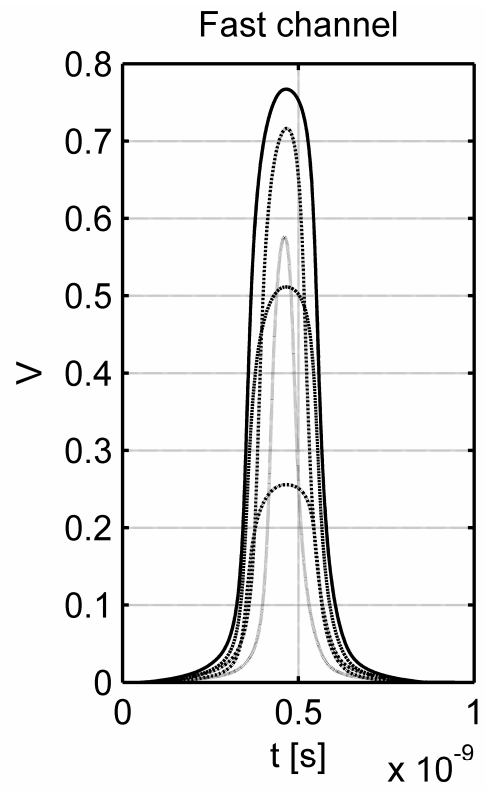

(c)

Fig 1. Replacing PAM pulses with PWM pulses. a) PAM and PWM input pulses. The PAM pulses have amplitudes of $0.33 \mathrm{~V}, 0.66 \mathrm{~V}$ and $1 \mathrm{~V}$. The PWM pulses have pulse-widths of $33 \%$, $66 \%$ and $100 \%$. $T_{s}=200 \mathrm{ps}$. b) Response of low-bandwidth example channel. c) Response of high-bandwidth example channel. 
This idea is shown in Fig. 1. The responses of a low-pass channel to a single PWM pulse and to a single SSF pulse are shown. This is done for two channels: one with a low bandwidth relative to the bit rate, and the other with a high bandwidth relative to the bit rate. We can see that for the first situation, where we need an equalizer, the channel response is indeed almost the same. For the situation with a fast channel, the response to the PWM signal is different from that to the PAM signal. However, equalization is not needed for a channel that is fast enough when compared to the bit rate.

It appears that the area under the pulse, whether it is a PWM or PAM pulse, does indeed determine the response of a 'slow' enough low-pass channel. The channel is sufficiently slow that it 'sees' each of the pulses as an impulse.

We now work with this same-area idea to make a multitap PWM filter that outputs the same area as a multitap SSF filter. To do this we first look at the SSF filter to determine which amplitude values it produces at its output as a function of the input bit stream. Next we replace these in the above fashion with PWM pulses and see whether the channel response is the same. The mathematics are dealt with in a later section; for now we look at the transient simulations to obtain an intuitive understanding.

A theoretical channel was used to perform the time domain simulations. The loss of the channel is monotonously increasing and is approximately $30 \mathrm{~dB}$ at the Nyquist frequency of $2.5 \mathrm{GHz}$. This theoretical channel is designed to have more pre-cursor ISI than e.g. $25 \mathrm{~m}$ RG-58CU so that a three-tap filter is necessary (one pre-cursor tap is added to the two-tap FIR). The channel loss is shown in Fig. 2(a), and the response to a 200ps pulse is shown in Fig. 2b.

We now look at the transient simulations. First, in Fig. 3(a), the output of a 3-tap symbolspaced FIR (SSF) filter is shown. In the same figure, the channel response to it is shown. Note that there are $2^{3}=8$ possible amplitude levels at the filter output. The bit rate is $5 \mathrm{~Gb} / \mathrm{s}$, which gives a symbol length (and bit length, assuming $2 \mathrm{PAM}$ ) of $\mathrm{T}_{\mathrm{s}}=200 \mathrm{ps}$. The tap settings used for this specific example are $w=\{-0.15,0.55,-0.29\}$. (These tap settings are optimized for the example channel.)

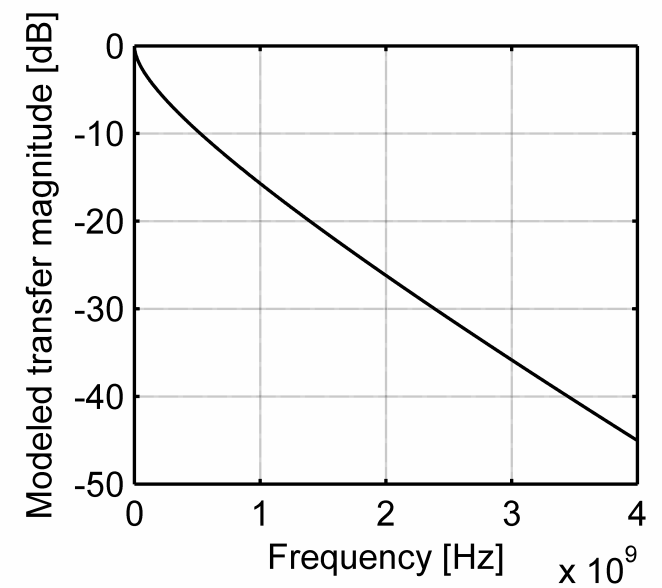

(a)

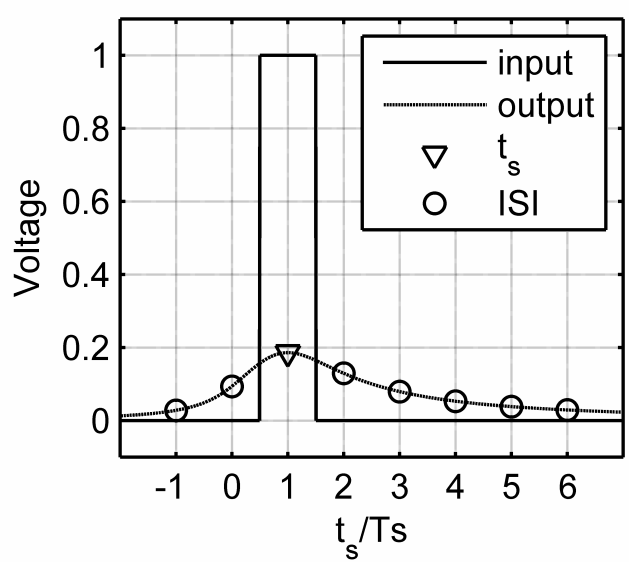

(b)

Fig. 2. Theoretical channel used for time domain simulations. (a) Channel loss. (b) pulse response $\left(T_{s}=200 \mathrm{ps}\right)$. 


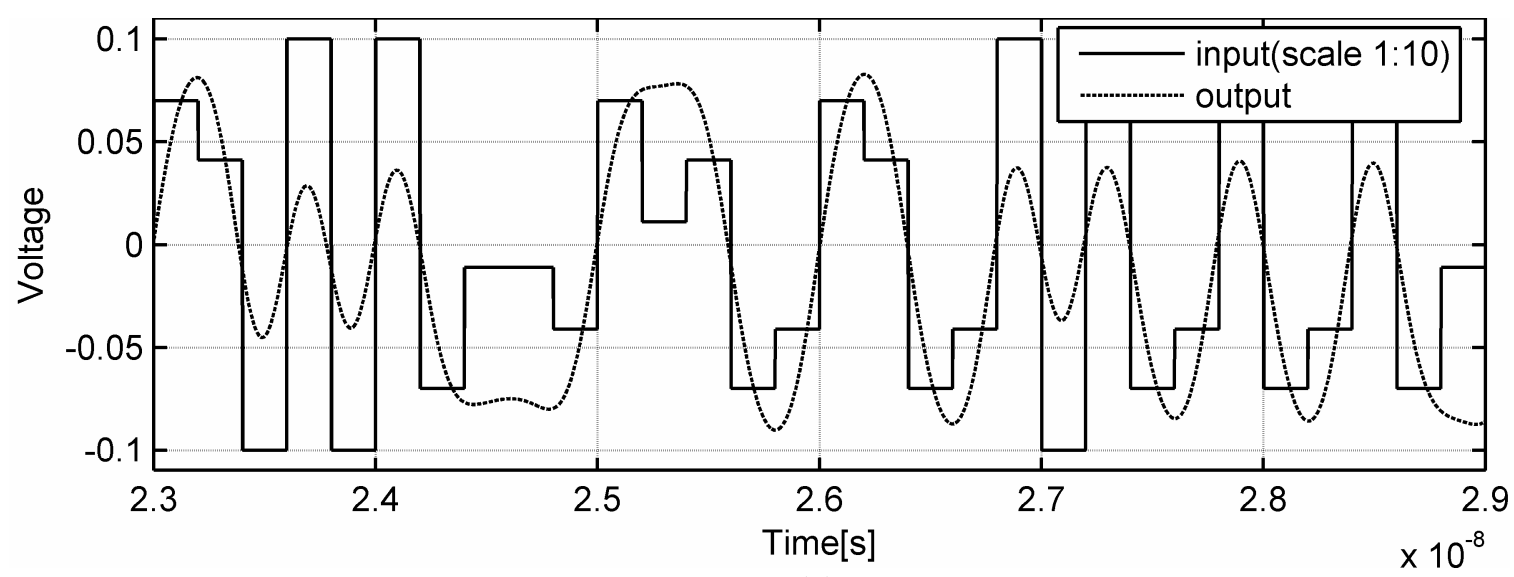

(a)

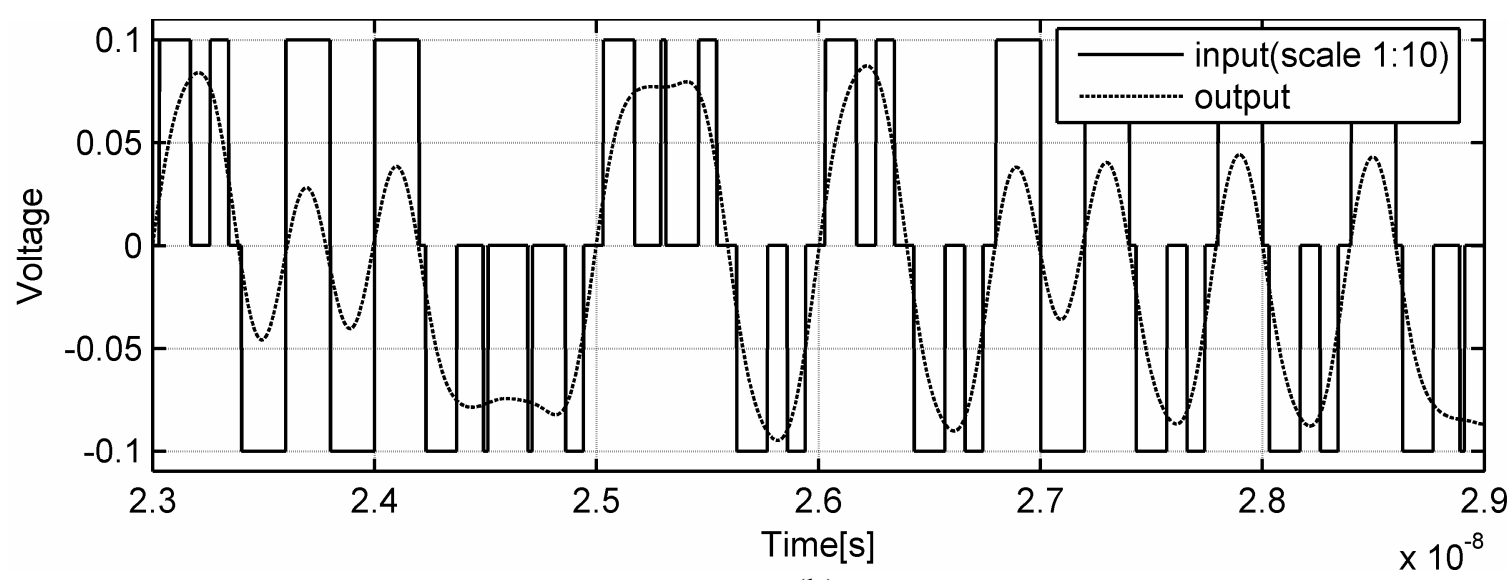

(b)

Fig. 3. Simulated response of example low-pass channel to 3-tap SSF and 3-tap PWM filter, and transmitter outputs (=channel inputs). (a) 3-tap SSF. (b) 3-tap, 3-voltage-level PWM.

It is now explained how the PWM filter output in Fig. 3(b) was made. To translate the amplitude modulated signal output of the SSF filter into a pulse-width modulated signal, and to be able to calculate the autocorrelation later, we first chop the signal up into 'singlets'. A precise mathematical definition of a singlet is given later. For now it is sufficient to know that the duration of a singlet is equal to the symbol duration $T_{s}(=200 \mathrm{ps}$ in our example), and that the edges between singlets are at those points in time where the SSF signal is allowed to change in amplitude. For example, looking at Fig. 3(a), we can see that there are five singlets between 25ns and 26ns. The same goes for Fig. 3(b), except that those singlets have a different shape. The amplitude of the SSF output is fixed during one singlet (Fig. 3(a)) because it is a symbol-spaced filter. The pulse-width of the PWM filter is fixed during one singlet (Fig. 3(b)) because it is also a symbol-spaced filter. Note that there are $2^{3}=8$ possible singlets at the filter output of the 3-tap PWM filter.

To calculate the singlets for the PWM filter, we convert each SSF singlet into a PWM singlet with an equal area, as was conceptually shown in Fig. 1. We make sure that the area under the PWM 'spikes' is the same as under the 'flat tops' of the SSF filter. The polarity of the SSF singlet is preserved by letting the PWM pulse go to either $1 \mathrm{~V}$ or $-1 \mathrm{~V}$, depending on the polarity of the SSF singlet. This PWM filter transmits three output levels (-1V, $0 \mathrm{~V}$ and $1 \mathrm{~V})$, and therefore we call it 3PWM. 


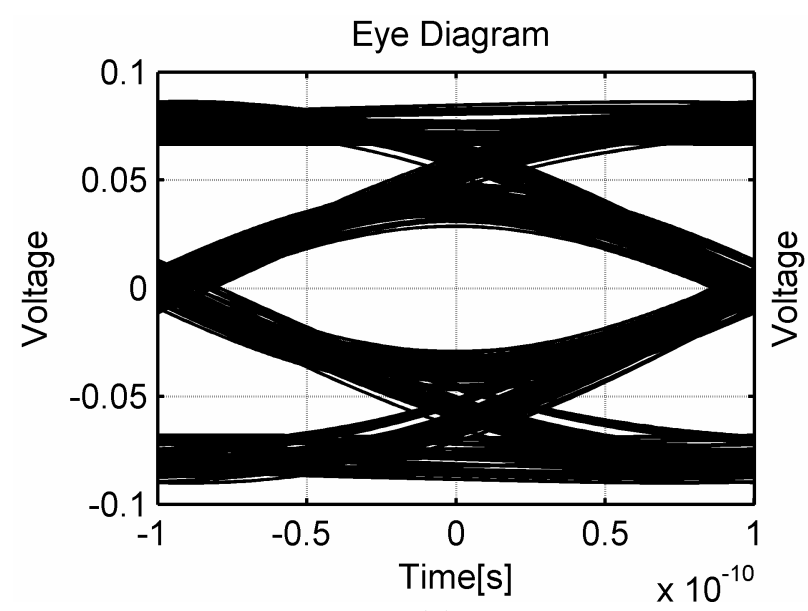

(a)

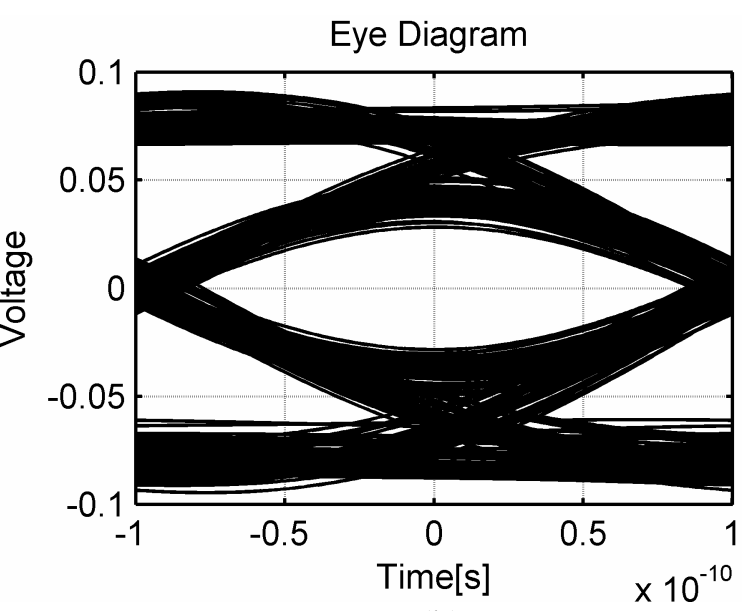

(b)

Fig. 4. Eye diagrams of response of example channel to 3-tap SSF input and to 3-tap PWM input, $\mathrm{T}_{\mathrm{s}}=200$ ps. (a) 3-tap SSF. (b) 3-tap, 3-voltage-level PWM.

As hoped, the channel response is almost the same as that to the 3-tap SSF signal, even though the transmitter output has quite a different shape. The eye diagrams in Fig. 4 confirm this. The eye diagram for the 3PWM filter looks practically the same as that for 3-tap SSF.

In conclusion, we have seen that we can obtain a transient cable response nearly identical to that of a SSF filter by replacing the PAM singlets with PWM singlets, using the same-area approach. The channel is successfully equalized. A prerequisite is that the channel has a lowpass characteristic.

\subsubsection{2-level, 3-tap PWM}

It might not be so straightforward to implement the 3-voltage-level PWM scheme presented above in a transmitter. For example, in a voltage mode implementation it would require 3 power supply rails. Therefore, it would be more convenient to have two instead of three allowed levels at the output of our PWM transmitter. This might make it easier to implement the circuit with a switching transmitter. Below we show how the number of output levels can be reduced to two without degrading the performance of the multitap PWM equalizer.

During one singlet, we now let the signal first go negative, then positive, and then negative again. We now need another translation from pulse amplitude to pulse-width that gives us two instead of three output levels. To find this new translation we can again use the same-area principle: the total area (positive and negative parts) should be equal to the area of the SSF singlet. We can perform these calculations and provide a new 2-voltage-level scheme. We use the term ' $2 \mathrm{PWM}$ ' because it outputs two voltage levels: $-1 \mathrm{~V}$ and $+1 \mathrm{~V}$. In the next section we present these calculations. Here we first show the results of the transient simulations.

The simulated transient response of the example channel to the 2PWM scheme is given in Fig. 5(b). For comparison, the channel response to the 3PWM scheme is repeated in Fig. 5(a). Both the 2PWM and 3PWM schemes are 3-tap filters; they only differ in the number of output levels. The eye diagrams are compared in Fig 6. As can be seen, the channel responses are almost identical. 


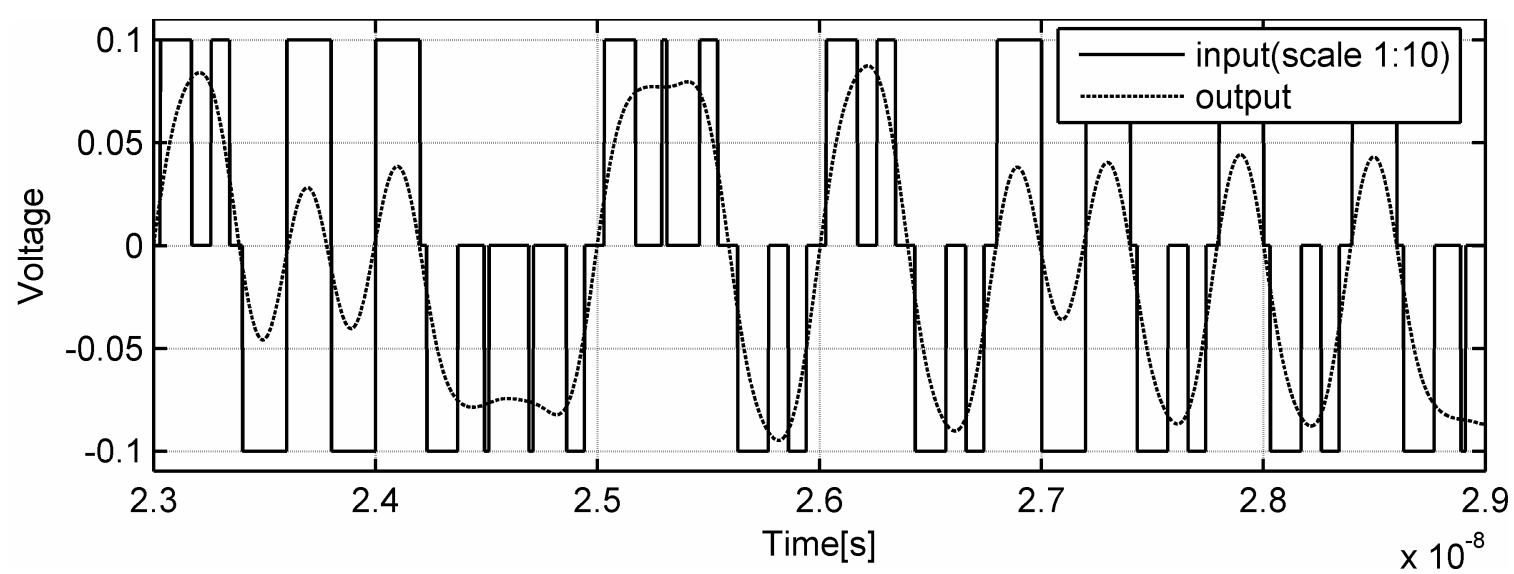

(a)

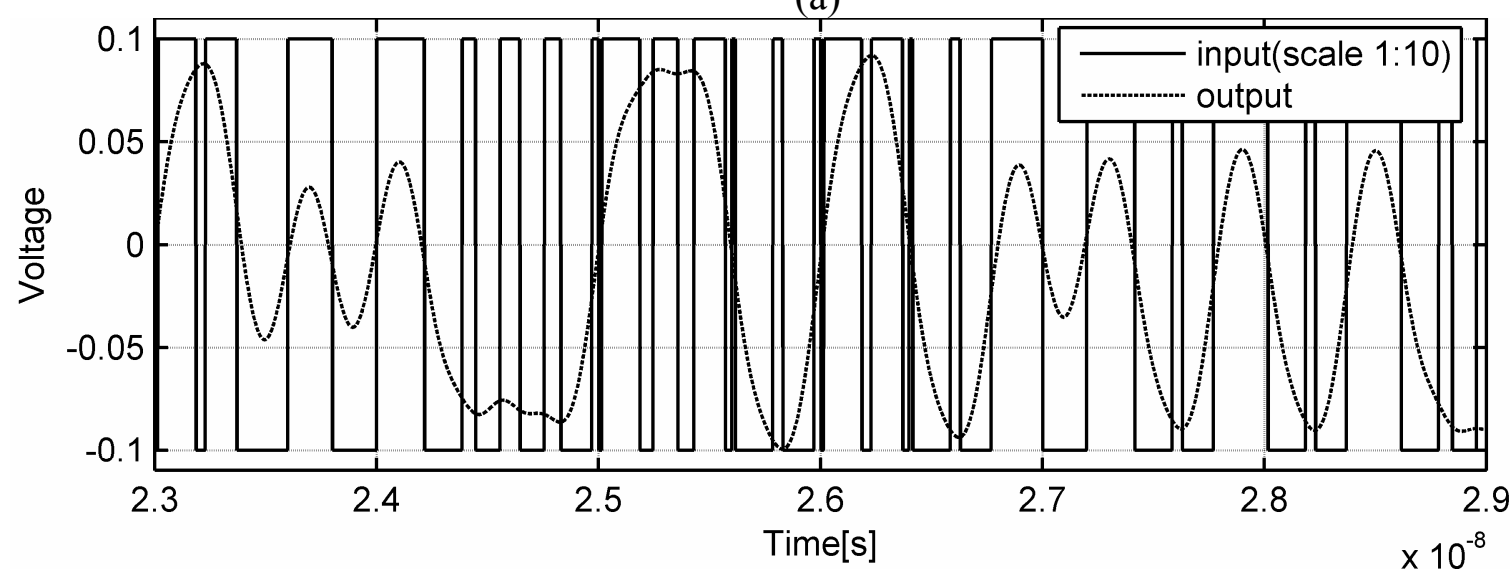

(b)

Fig. 5. Simulated transient response of example channel to 3PWM and to 2PWM, and transmitter outputs (=channel inputs), $\mathrm{T}_{\mathrm{s}}=200 \mathrm{ps}$. Both PWM filters have three taps. (a) 3-level PWM (3PWM). (b) 2-level PWM (2PWM).

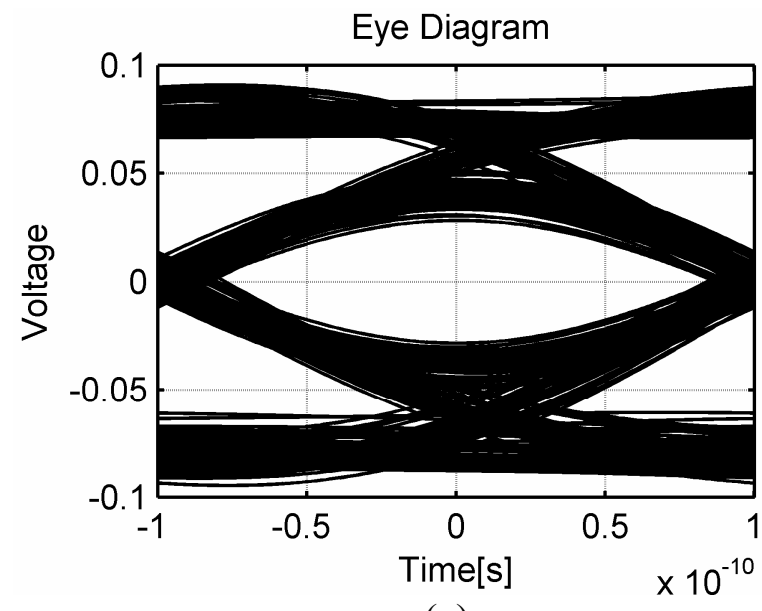

(a)

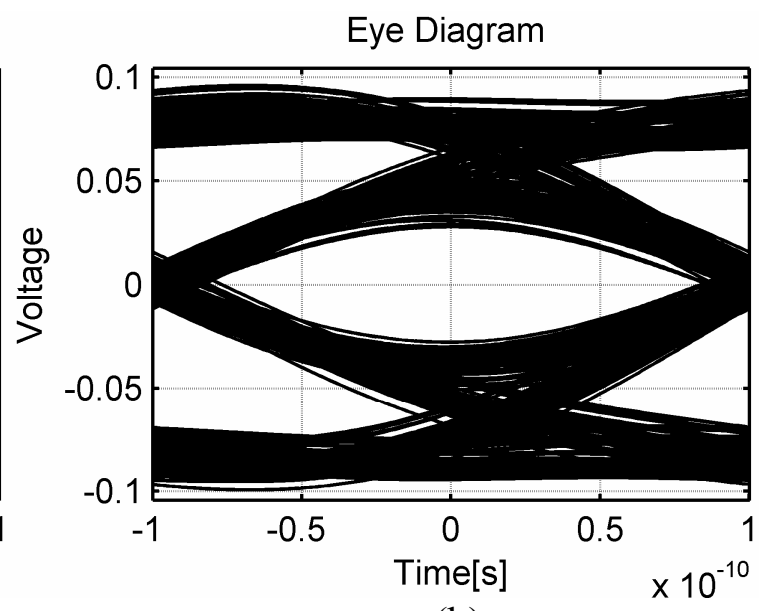

(b)

Fig. 6. Eye diagrams of response of example channel to 3PWM input and to 2PWM input, $\mathrm{T}_{\mathrm{s}}=200$ ps. (a) 3-tap, 3-level PWM (3PWM) (b) 3-tap, 2-level PWM (2PWM)

We can conclude that the 3-tap SSF, the 3-tap 3-level PWM (3PWM), and 3-tap 2-level PWM (2PWM) schemes all result in almost the same output eye diagram, even though the TX shapes are vastly different. As shown in the time domain simulations, the channel responds in the same way to both the PWM and the SSF pre-emphasis filters due to the channel's lowpass behavior. 
Furthermore, we conclude that it was straightforward to find the duty-cycles because they were derived from the singlet heights of the SSF filter using a 'same-area' approach.

\subsection{Power spectral density (PSD) functions of the multitap PWM filters}

Now that we have established an intuitive time-domain understanding of the multitap PWM filters, we analyze the filter from a frequency-domain point of view. The power spectral density (PSD) functions of the PWM filters are calculated and compared to the PSD of the 3-tap SSF filter.

We can obtain the power spectral density (PSD) in the following way:

1) Calculate the autocorrelation $R_{x x}(\tau)$ (subsection 5.3.1).

2) Take the Fourier transform $F\{\}$ of the autocorrelation to calculate the PSD (subsection 5.3.2):

$$
P S D=F\left\{R_{x x}(\tau)\right\}
$$

\subsubsection{Autocorrelation method}

In this subsection, we calculate the autocorrelations of the 3-tap PWM filters. We use the method first to calculate the autocorrelation of the well-known 3-tap SSF filter. This might seem cumbersome, because for the FIR filter we could more easily use (for example) a z-transform, but in this way the result (and the correctness of the method) can be checked against the known PSD function of the 3-tap SSF filter. For calculating the autocorrelation of the PWM filters, we cannot use methods commonly used for FIR filters, because of the timevarying nature of the PWM filter. Therefore we need to devise a calculation method that accepts pulse-width modulated signals. This method uses the 'singlets', as described above. It can be extended to more filter taps. As an additional check, we compare the calculated autocorrelations with statistical simulations.

This subsection is divided as follows. First, in 5.3.1.1, we explain how to calculate the singlets needed for our method, and as an example we calculate the singlet shapes for the 3-tap SSF filter. Next, in 5.3.1.2, it is explained how to calculate the autocorrelation from these singlets. In 5.3.1.3, we calculate the singlet shapes for the 3PWM and 2PWM schemes. Finally, in 5.3.1.4, we use the method to calculate the autocorrelations of 3PWM, 2PWM, 2-tap SSF and polar NRZ.

\subsubsection{From bits to singlets}

As explained above, we cut the transmitted transient data signal for which the autocorrelation is to be calculated into singlets $y(t)$, each of length $T_{s}$ (=symbol duration and bit duration, because 2PAM is used). The singlets $y(t)$ are defined as follows. They have a width of $T_{s}$, are zero outside of the interval $\left(0, T_{s}\right)$ and inside that interval they are a function of time $t$ :

$$
y(t)=\left\{\begin{array}{cc}
0, & t<0, \\
f(t), & 0<t<T_{s}, \\
0, & t>T_{s} .
\end{array}\right.
$$




\begin{tabular}{|c|c|c|}
\hline Singlet name & Singlet number & Bit sequence $b_{n-1} \ldots b_{n+1}$ \\
\hline $\mathrm{A}$ & $y_{1}(t)$ & $-1 \quad 1-1$ \\
\hline $\mathrm{B}$ & $y_{2}(t)$ & $\begin{array}{lll}-1 & 1 & 1 \\
\end{array}$ \\
\hline $\mathrm{C}$ & $y_{3}(t)$ & $11-1$ \\
\hline $\mathrm{D}$ & $y_{4}(t)$ & $\begin{array}{lll}11 & 1 \\
\end{array}$ \\
\hline$-\mathrm{A}$ & $y_{5}(t)$ & $1-11$ \\
\hline$-\mathrm{B}$ & $y_{6}(t)$ & $1-1-1$ \\
\hline$-\mathrm{C}$ & $y_{7}(t)$ & $-1-1 \quad 1$ \\
\hline$-\mathrm{D}$ & $y_{8}(t)$ & $-1-1-1$ \\
\hline
\end{tabular}

Table 1. Singlets and represented bit sequences.

In a 3-tap filter, the singlet shape is a function of three bits. At the output of a 3-tap SSF filter, there are 8 possible singlets, with 8 different voltage amplitudes $\left(2^{3}=8\right.$ combinations $)$, as a function of the three-bit sequence $b_{n-1} \ldots b_{n+1}$, where $n$ is the bit index from the interval $(-\infty, \infty)$. The same applies to the 3-tap PWM filter: there are 8 allowed pulse-widths at the filter output (or 4 pulse-widths and 2 polarities). In the case of the SSF filter, each singlet has a specific height (and fixed width of 1 bit time) and in the case of PWM each singlet has a specific width and polarity (and a fixed amplitude of $1 \mathrm{~V}$ ). The advantage of this method is that the actual singlet shape $f(t)$ does not matter. It can be either a SSF or PWM singlet - or anything else.

We start with four of the eight singlets A, B, C and D and we can then make the other four by flipping them over the t-axis to obtain $-\mathrm{A},-\mathrm{B},-\mathrm{C}$ and $-\mathrm{D}$. This symmetry eases the calculation of the autocorrelation. (There are other, non-symmetrical configurations possible but these lead to more complex autocorrelation calculations while not offering a clear benefit.)

In Table 1, the assignment of singlet names to bit sequences is shown. For example, if the bit sequence $b_{n}$ to be transmitted is equal to $\{\ldots,-1,1,-1,1,1, \ldots\}$, then the singlet output of the 3 -tap filter would be $\{\ldots, \mathrm{A},-\mathrm{A}, \mathrm{B}, \ldots\}$, where A-D stand for the singlets in Table 1.

To give an example, and for later use, we now calculate the exact shape of the singlets for a 3tap SSF filter. The mathematical description of the singlets for the 3-tap SSF filter is:

$$
y_{S S F}(t)=\left\{\begin{array}{lc}
0, & t<0, \\
\alpha, & 0<t<T_{s}, \\
0, & t>T_{s},
\end{array}\right.
$$

where the singlet amplitude $\alpha$ is given by:

$$
\alpha=w_{1} \cdot b_{n+1}+w_{2} \cdot b_{n}+w_{3} \cdot b_{n-1},
$$

where $w$ represents the tap weight values.

The calculated singlet shapes for a 3-tap SSF filter are illustrated in Fig. 7 below, for tap settings $w=\{-0.15,0.55,-0.29\}$. The amplitude is normalized to $+/-1 \mathrm{~V}$ to allow a samevoltage-headroom comparison to the PWM filter. 
A: $-11-1$

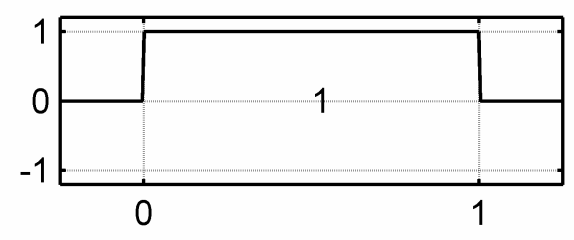

B: -111
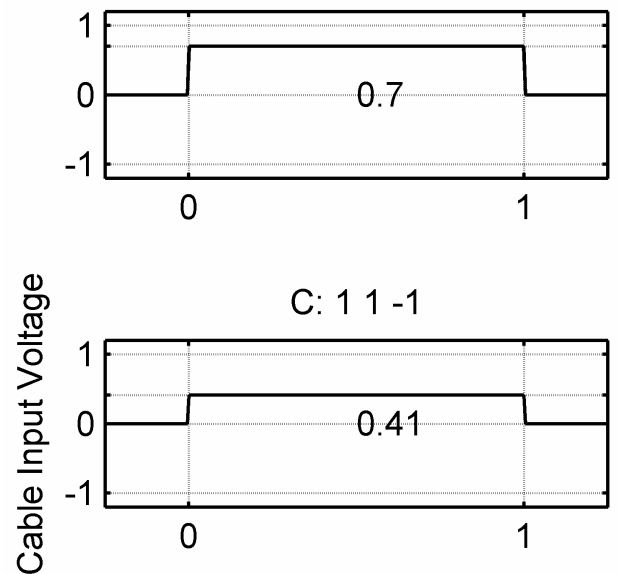

D: 111

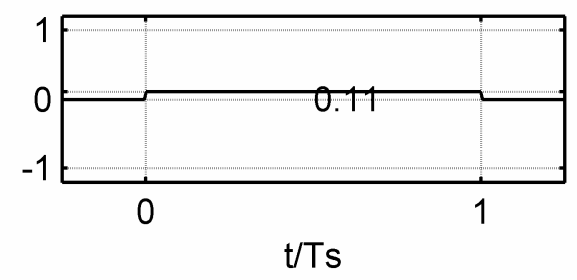

$-\mathrm{A}: 1-11$

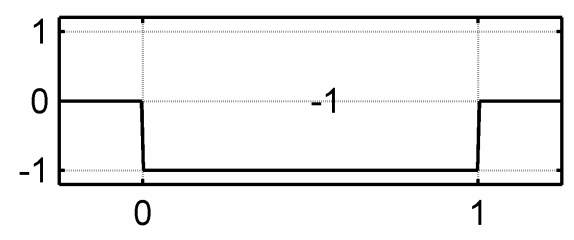

-B: $1-1-1$
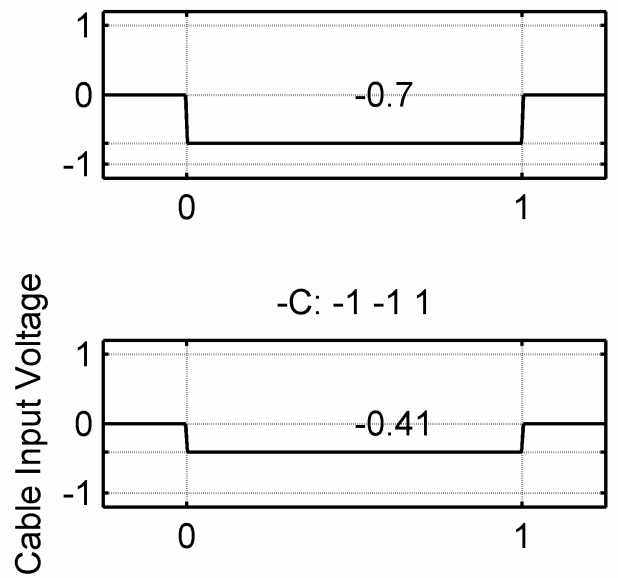

$-\mathrm{D}:-1-1-1$

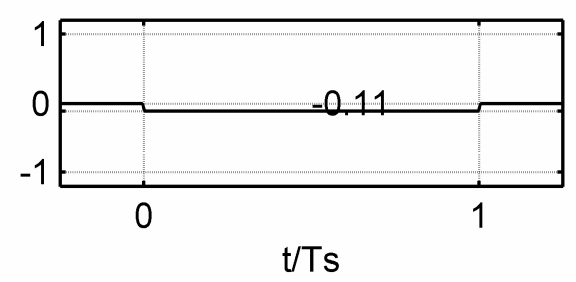

Fig. 7. All possible singlets for a three tap SSF filter, as a function of the 3-bit sequence. In the middle of each singlet, $\alpha$ is printed.

\subsubsection{Autocorrelation from singlets}

As is well known, the formula for calculating the autocorrelation of a signal $x(t)$ as a function of time shift $\tau$ is [Couch]:

$$
R_{x x}(\tau)=\lim _{T \rightarrow \infty} \frac{1}{2 T} \int_{-T}^{T} x(t) x(t+\tau) d t .
$$

We assume that the signal is ergodic; its time-average is equal to its ensemble average. The autocorrelation for our 3-tap symbol-spaced filter has a total width of $6 T_{s}$; it is zero outside of the interval for $\tau$ of $\left(-3 T_{s}, 3 T_{s}\right)$. This is because the singlets have a width of $T_{s}$ and the filter is a 3 -tap symbol-spaced filter.

Using the singlet shapes A-D together with the probabilities of their occurrence, we can calculate the autocorrelation for the filtered data stream. Because the singlets are a function of three bits (and each singlet is only one bit wide) not all combinations of singlets are allowed to follow each other. For example, the singlet corresponding to bit sequence $\{-1,1,-1\}$ (' $A$ ') can only be followed by a singlet corresponding to $\left\{1,-1,{ }^{*}\right\}$, where * denotes either +1 or -1 
('-A' or '-B'); e.g. 'D' (corresponding to $\{1,1,1\})$ is not possible. To understand this, shift the first bit sequence one bit to the left.

To obtain the autocorrelation, we need to check all the possible combinations and their probability of occurrence. Then, to calculate the autocorrelation using the singlets, we split the autocorrelation function into five (partially overlapping) parts:

-the first part $\left(R_{2}\left(\tau+2 T_{s}\right)\right)$ around a time shift of $-2 T_{s}$

-the second part $\left(R_{1}\left(\tau+T_{s}\right)\right)$ around a time shift of $-T_{s}$

-the third part $\left(R_{0}(\tau)\right)$ around a zero time shift

-the fourth part $\left(R_{l}\left(-\tau-T_{s}\right)\right)$ around a time shift of $T_{s}$

-and the fifth part $\left(R_{2}\left(-\tau-2 T_{s}\right)\right)$ around a time shift of $2 T_{s}$.

Each of the five parts has a width of $2 T_{s}$ because the width of each singlet is $T_{s}$. The five parts are illustrated in Fig. 8 for the 3-tap SSF filter.

The autocorrelation $R_{x x}(\tau)$ is symmetrical around $\tau=0$, and is composed of the three components $R_{0}(\tau), R_{1}(\tau)$ and $R_{2}(\tau)$ as follows:

$$
R_{x x}(\tau)=R_{2}\left(\tau+2 T_{s}\right)+R_{1}\left(\tau+T_{s}\right)+R_{0}(\tau)+R_{1}\left(-\tau-T_{s}\right)+R_{2}\left(-\tau-2 T_{s}\right) .
$$

The components $R_{0}(\tau), R_{1}(\tau)$ and $R_{2}(\tau)$ are defined as follows. First, $R_{0}(\tau)$ is defined as:

$$
R_{0}(\tau)=\frac{1}{8} \sum_{i=1}^{8}\left(\frac{1}{T_{s}} \int_{0}^{T_{s}} y_{i}(t) y_{i}(t-\tau) d t\right),
$$

where $y_{x}(t)$ are the singlets as defined in Table 1.

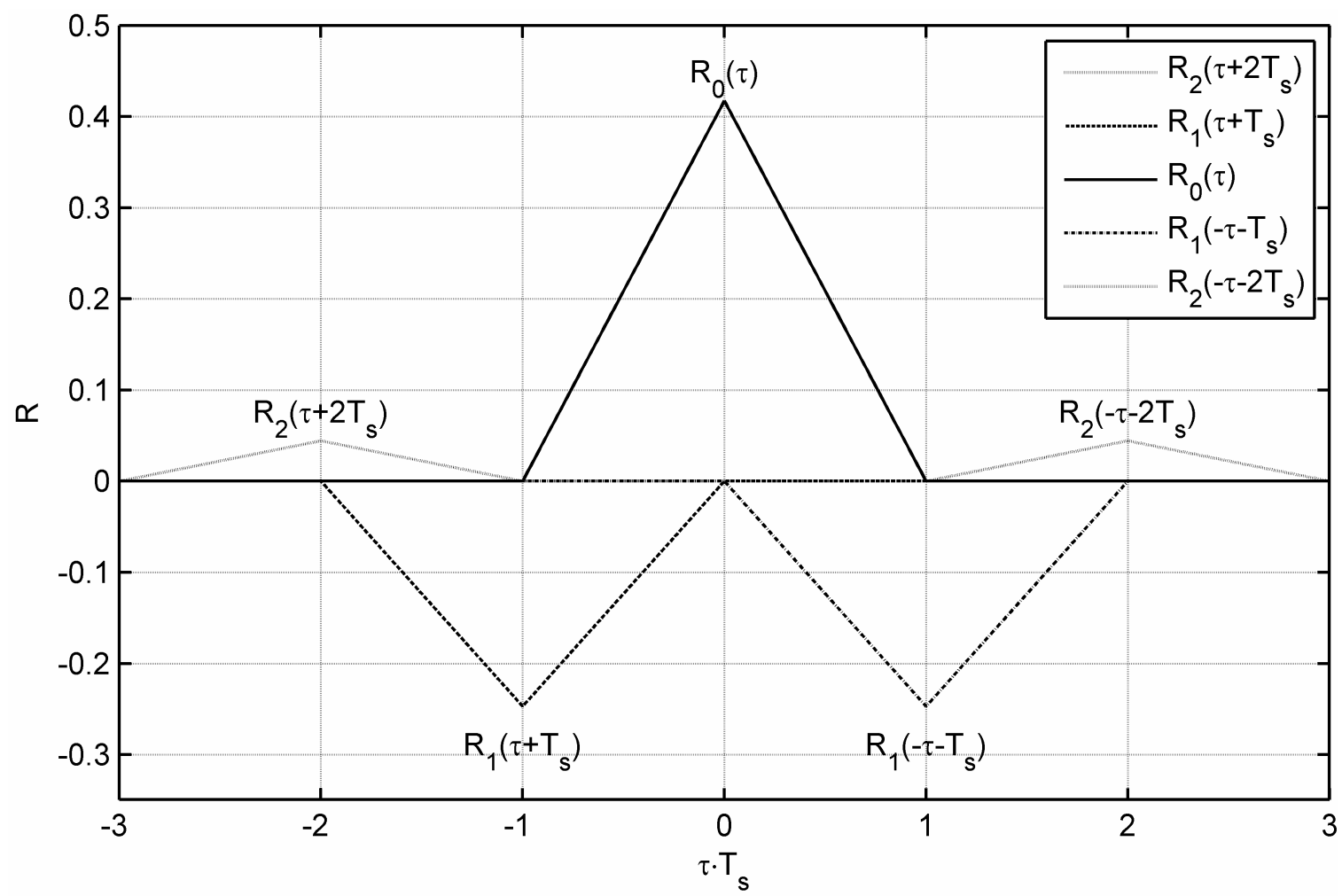

Fig. 8. Autocorrelation components $R_{0}(\tau), R_{1}(\tau)$ and $R_{2}(\tau)$ for the 3-tap SSF filter. 
Next, $R_{l}(\tau)$ is defined as:

$$
R_{1}(\tau)=\frac{1}{16} \sum_{i=1}^{8}\left(\sum_{j=1}^{2}\left(\frac{1}{T_{s}} \int_{0}^{T_{s}} y_{i}(t) y_{v_{1}(i, j)}(t-\tau) d t\right)\right),
$$

where $y_{v l(i, j)}$ is the $j$ th allowed singlet to follow singlet $y_{i}$. Appendix $\mathrm{C}$ lists the values for $y_{v 1(i, j)}$. The values for $j$ are chosen from the interval $[1,2]$ because, as explained above, for each of the eight $(i=1 . .8)$ singlets, only two singlets are allowed to follow it. This is because the bit sequence is shifted to the left by one bit. There are $8 \cdot 2=16$ allowable combinations in total.

Finally, $R_{2}(\tau)$ is defined as:

$$
R_{2}(\tau)=\frac{1}{32} \sum_{i=1}^{8}\left(\sum_{j=1}^{4}\left(\frac{1}{T_{s}} \int_{0}^{T_{s}} y_{i}(t) y_{v_{2}(i, j)}(t-\tau) d t\right)\right)
$$

where $y_{v 2(i, j)}$ is the $j$ th allowed singlet to follow singlet $y_{i}, 2 T_{s}$ after the start of this first singlet. Appendix $\mathrm{C}$ lists the values for $y_{v 2(i, j)}$. The values for $j$ are chosen from the interval $[1,4]$ because the bit sequence is shifted to the left by two bits, so that for each of the eight $(i=1 . .8)$ singlets there are now four singlets that are allowed to follow it, $2 T_{s}$ after its start. There are $8 \cdot 4=32$ allowable combinations in total.

The above equations for $R_{0}(\tau), R_{l}(\tau)$ and $R_{2}(\tau)$ can be generalized into one equation for $R_{k}(\tau)$ :

$$
R_{k}(\tau)=\frac{1}{8 \cdot 2^{k}} \sum_{i=1}^{8}\left(\sum_{j=1}^{2^{k}}\left(\frac{1}{T_{s}} \int_{0}^{T_{s}} y_{i}(t) y_{v_{k}(i, j)}(t-\tau) d t\right)\right) .
$$

In Appendix $\mathrm{C}$, the complete calculation is given. The results are reproduced here. The components $R_{0}(\tau), R_{l}(\tau)$ and $R_{2}(\tau)$ are given by, respectively:

$$
\begin{gathered}
R_{0}(\tau)=\frac{1}{4}\left(R_{A A}+R_{B B}+R_{C C}+R_{D D}\right), \\
R_{1}(\tau)=\frac{1}{8}\left(-R_{A A}-R_{A B}+R_{B C}+R_{B D}-R_{C A}-R_{C B}+R_{D C}+R_{D D}\right),
\end{gathered}
$$

and

$$
\begin{aligned}
& R_{2}(\tau)=\frac{1}{16}\left(R_{A A}+R_{A B}-R_{A C}-R_{A D}-R_{B A}-R_{B B}+R_{B C}+R_{B D}\right. \\
& \left.+R_{C A}+R_{C B}-R_{C C}-R_{C D}-R_{D A}-R_{D B}+R_{D C}+R_{D D}\right) .
\end{aligned}
$$

In the above equations, a shorthand notation is used, e.g. $R_{A B}$ means:

$$
R_{A B}=\int_{0}^{T_{s}} y_{1}(t) y_{2}(t+\tau) d t
$$

where $y_{1}(t)$ is the singlet ' $\mathrm{A}$ ' and $y_{2}(t)$ is the singlet ' $\mathrm{B}$ ' (see Table 1 ). 


\subsubsection{Calculation of singlets for the PWM filters}

Now, a mathematical description is given of the singlets $A, B, C$ and $D$ for the $3 \mathrm{PWM}$ and 2PWM schemes.

\section{Singlets for 3PWM}

The mathematical description of the 3PWM singlets is

$$
y_{3 P W M}(t)=\left\{\begin{array}{cc}
0, & t<(1-|\alpha|) T_{s} / 2, \\
\operatorname{sign}(\alpha), & (1-|\alpha|) T_{s} / 2<t<(1+|\alpha|) T_{s} / 2, \\
0, & t>(1+|\alpha|) T_{s} / 2 .
\end{array}\right.
$$

We can use the same variable $\alpha$ as for the 3-tap SSF filter, but now it denotes the pulse width and pulse polarity instead of the pulse height. It falls into the interval of [-1,1], because of the normalization that was done. As previously stated, the parameter $\alpha$ contains information about the pulse-width and its polarity. The absolute value of $\alpha$, which is a number from the interval $[0,1]$, corresponds to the pulse-width $(0-100 \%)$ and the sign of $\alpha$ corresponds to the pulse polarity.

A: $-11-1$

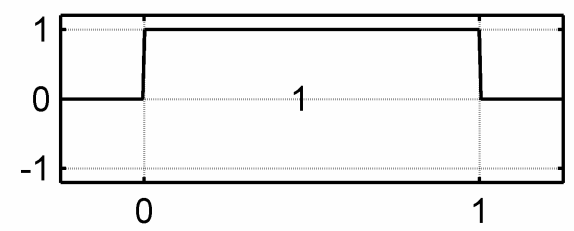

B: -111
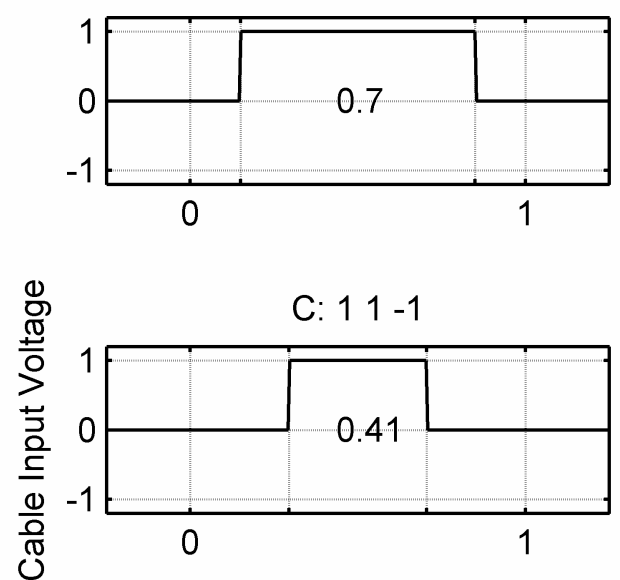

D: 111

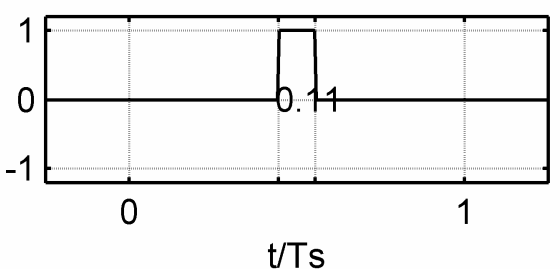

$-A: 1-11$

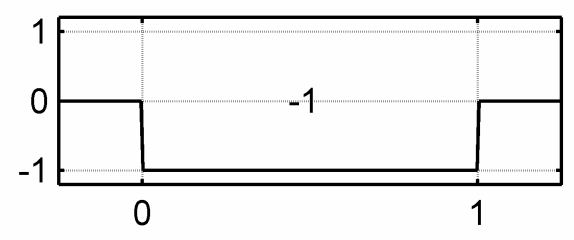

-B: $1-1-1$
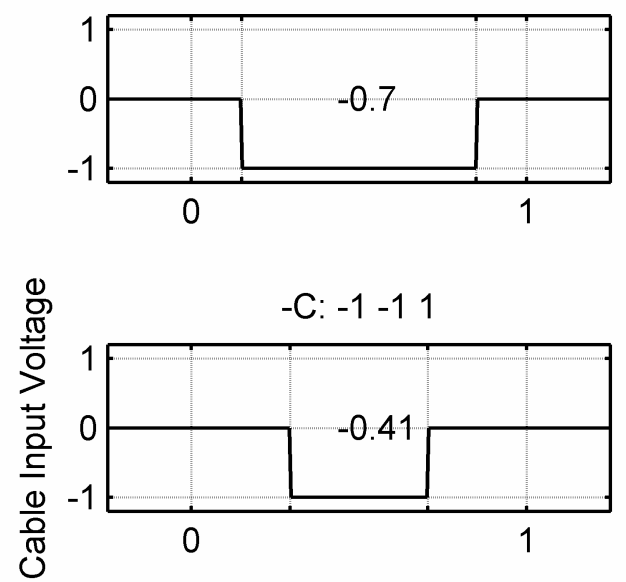

$-D:-1-1-1$

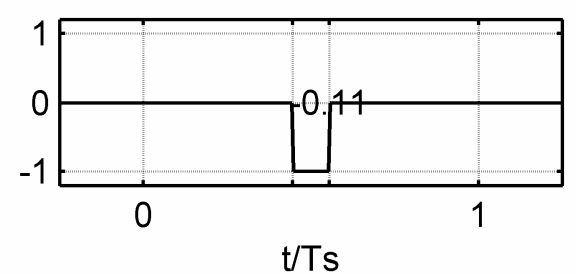

Fig. 9. All possible output singlet shapes for 3-tap 3-voltage-level PWM pre-emphasis (3PWM). $\alpha$ is printed in the middle of each singlet. 
In Fig. 9, the singlets for 3PWM are shown. It can easily be proven that the areas under the 3PWM and SSF singlets (see Fig. 7) are equal.

\section{Singlets for 2PWM}

As mentioned earlier, we would like to reduce the number of output levels of our PWM transmitter to two levels ( $1 \mathrm{~V}$ and $-1 \mathrm{~V})$ instead of three. The total area is now a sum of the positive and negative parts, as described above. To keep the area equal to the SSF area, the pulse-width needs to be different from that of the 3PWM scheme. Again, we use a single variable to denote both the pulse-width and its polarity. The absolute value of this parameter $\psi$ is the pulse-width and its sign is the pulse polarity. This scheme is termed 2PWM because it has two output voltage levels.

The definition of the 2PWM singlets is as follows:

$$
y_{2 P W M}(t)=\left\{\begin{array}{cc}
0, & t<0, \\
-\operatorname{sign}(\psi), & 0<t<(1-|\psi|) T_{s} / 2, \\
\operatorname{sign}(\psi), & (1-|\psi|) T_{s} / 2<t<(1+|\psi|) T_{s} / 2, \\
-\operatorname{sign}(\psi), & (1+|\psi|) T_{s} / 2<t<T_{s}, \\
0, & t>T_{s},
\end{array}\right.
$$

where $\operatorname{sign}(\psi)=\operatorname{sign}(\alpha)$, per definition, and $\psi$ is chosen from the intervals $[-1,-0.5]$ and $[0.5,1]$. Values in the interval $(-0.5,0.5)$ are not allowable. Using these intervals, we can achieve an equal summed area as that in the 3PWM scheme, as is shown below.

The total area under the new pulse is equal to:

$$
A_{n 1}=\int_{-\infty}^{\infty} y_{n 1}(t) d t=\operatorname{sign}(\psi)\left(-\int_{0}^{(1-|\psi|) T_{s} / 2} 1 d t+\int_{(1-|\psi|) T_{s} / 2}^{(1+|\psi|) T_{s} / 2} 1 d t-\int_{(1+|\psi|) T_{s} / 2}^{T_{s}} 1 d t\right)=(2 \psi-\operatorname{sign}(\psi)) T_{s} .
$$

We calculate how to translate from $\alpha$ to $\psi$, using the same-area approach:

$$
\begin{gathered}
A_{n 1}=A_{3 P W M}, \\
(2 \psi-\operatorname{sign}(\psi)) T_{s}=\alpha T_{s}, \\
|\psi|=(|\alpha|+1) / 2,
\end{gathered}
$$

(and $\operatorname{sign}(\psi)=\operatorname{sign}(\alpha)$, per definition, as said above). Note how the interval for $\alpha$ of $[-1,1]$ indeed leads to an interval for $\psi$ of $[-1,-0.5]$ and $[0.5,1]$. As soon as $\alpha<0$, the 2PWM pulse is flipped around the t-axis, because of its sign definition, maintaining the symmetry between A,B,C,D and -A,-B,-C,-D singlets. The resulting singlet shapes are shown in Fig. 10. 
A: $-11-1$

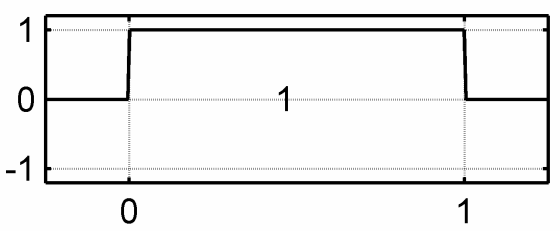

B: -111
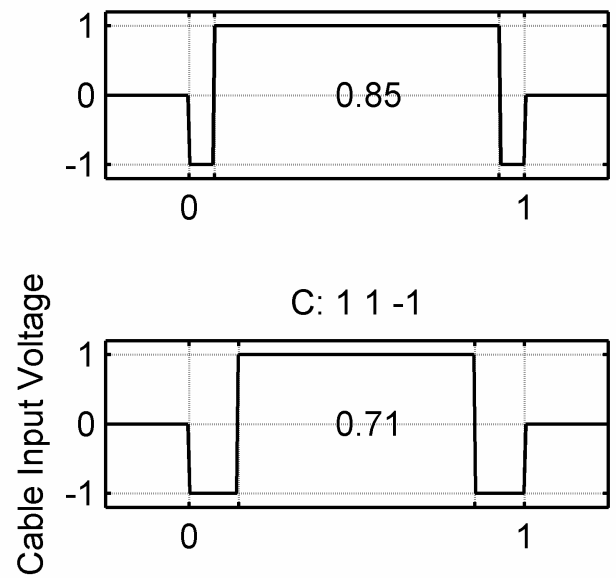

D: 111

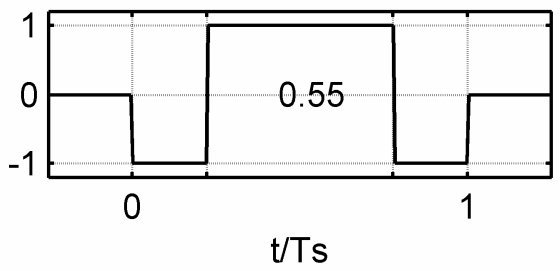

$-A: 1-11$

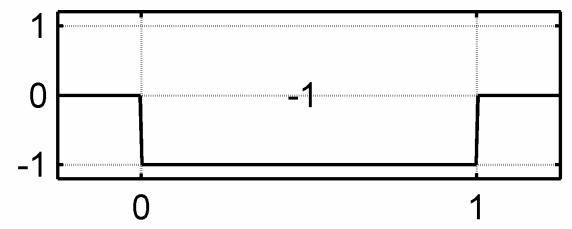

-B: $1-1-1$
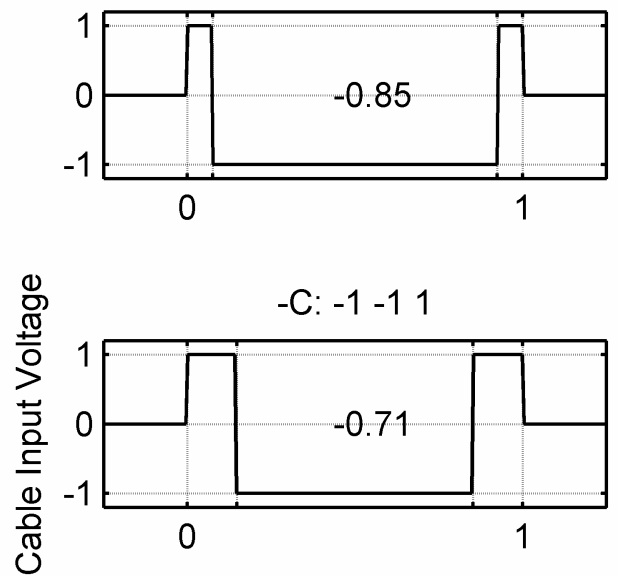

$-\mathrm{D}:-1-1-1$

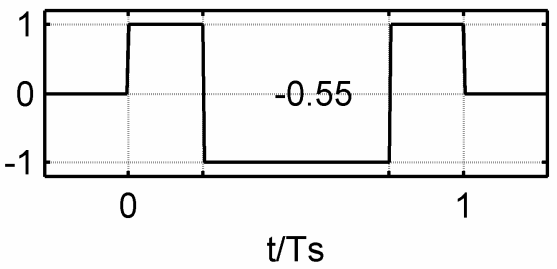

Fig. 10. All possible output singlet shapes for 3-tap, 2-voltage-level PWM pre-emphasis $(2 \mathrm{PWM}) . \psi$ is printed in the middle of each singlet.

The translation is ambiguous at only one point, which is at $\alpha=0$ (where $\alpha$ has no sign). At this point, there are two possible singlet shapes for the $2 \mathrm{PWM}$ scheme, each with a $50 \%$ dutycycle. The first is negative, positive, and then negative $(\psi=0.5)$, and the second is exactly the opposite: first positive, then negative, then positive $(\psi=-0.5)$. We define here that in that case (which is rare), the sign of $\psi$ be equal to the sign of the most significant filter tap (the tap with the maximum absolute value).

\subsubsection{Autocorrelation results}

Knowing the description of all the singlet shapes, we can fill them into the previously given autocorrelation formula and calculate the autocorrelations. The method is used to calculate the autocorrelations for the 2PWM and 3PWM schemes. For comparison, also the autocorrelation for the 3-tap SSF filter output and for polar NRZ (2PAM, unequalized) is calculated.

To check the analytical calculations, we also compare the calculated autocorrelations to the results from a statistical simulation with 10,000 random symbols. 


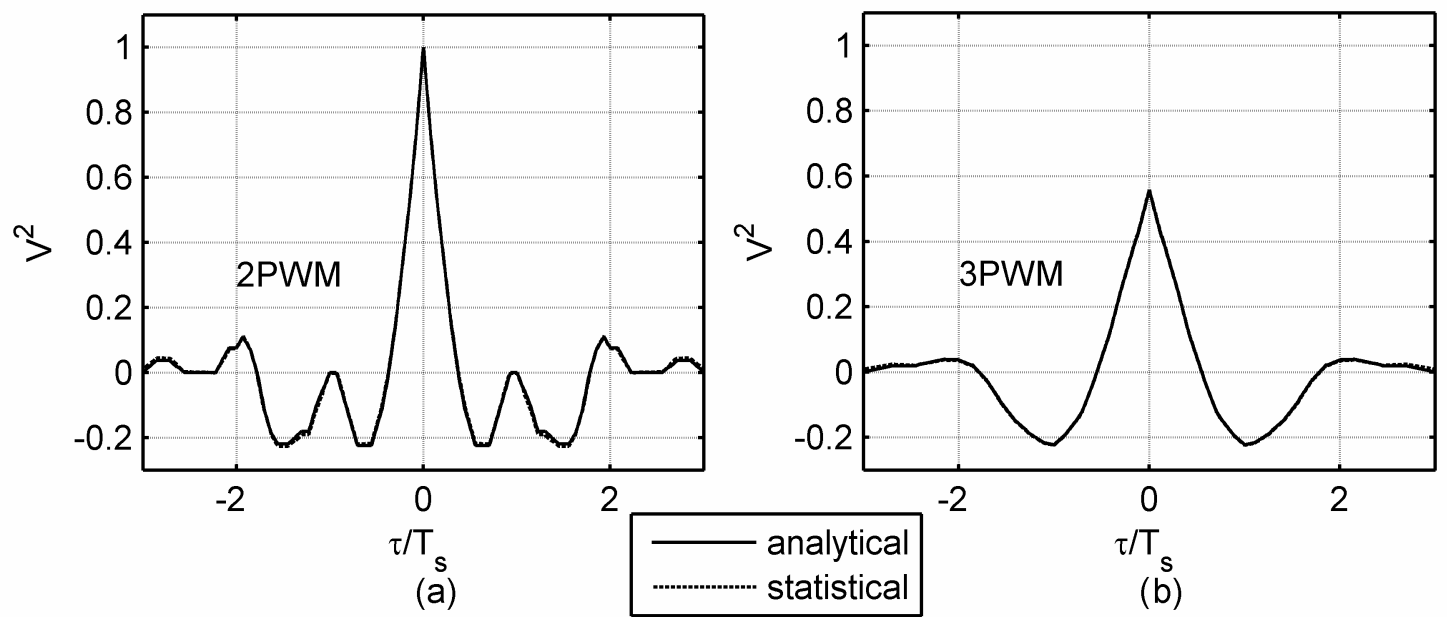

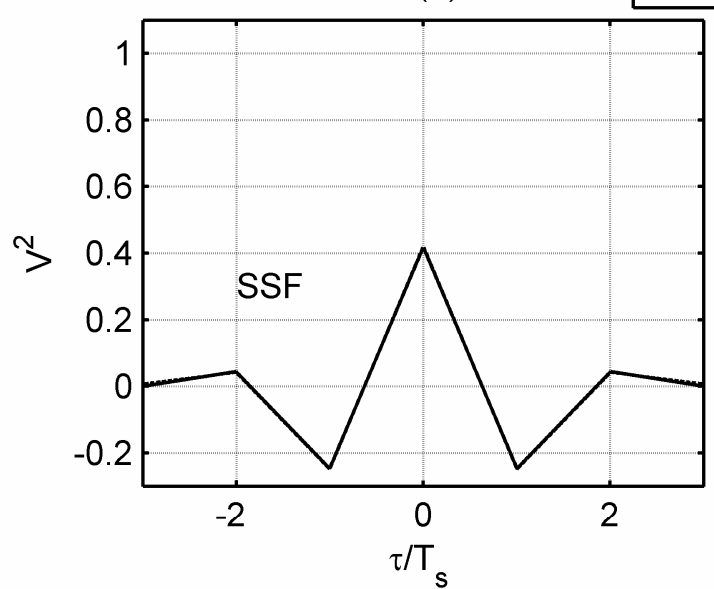

(c)

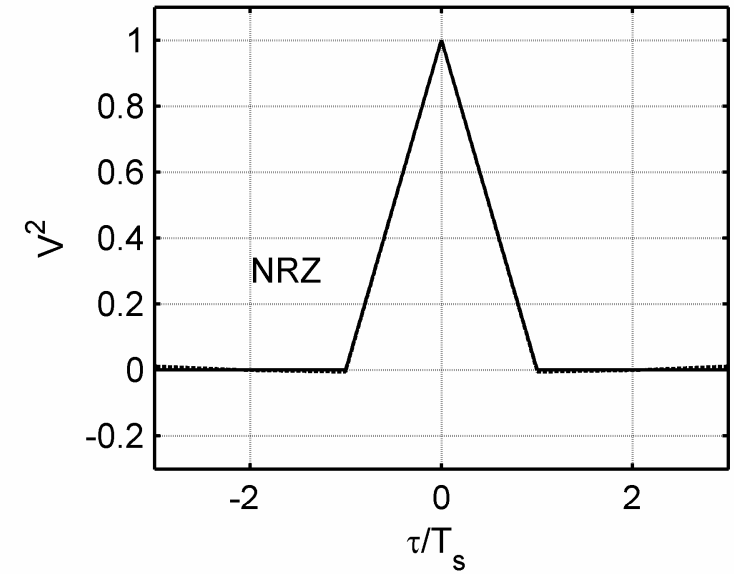

(d)

Fig. 11. Autocorrelations (from analytical and from statistical simulations). In some plots only the 'analytical' line can be seen because the 'statistical' line is right below it. (a) 2PWM (2level, 3-tap). (b) 3PWM (3-level, 3-tap). (c) 3-tap SSF. (d) Polar NRZ.

The results are shown in Fig. 11. The individual plots show the following autocorrelations:
a) 2PWM filter,
b) 3PWM filter,
c) 3-tap SSF filter,
d) polar NRZ.

The signals are normalized to a $+/-1 \mathrm{~V}$ supply voltage headroom. We see that $3 \mathrm{PWM}$ has an autocorrelation that very closely matches that of 3-tap SSF. The 2PWM autocorrelation shows more high-frequency components, due to the higher switching frequency.

\subsubsection{PSD functions}

In this subsection we describe the calculation of the PSD functions for the multitap PWM filters. We now have all the information to do this, using the autocorrelations calculated above.

As described above, we use the Fourier transform to obtain the PSDs from the autocorrelations. To check the analytical calculations, we again compare the calculated PSDs to the results from a statistical simulation with 10,000 random symbols. 
Fig 12 shows the PSDs for the following schemes:

(a) 2PWM filter

(b) 3PWM filter

(c) 3-tap SSF filter

(d) polar NRZ (2PAM, unequalized).

A smoothing filter was used on the statistically calculated PSDs. Small differences between the statistical simulations and the analytically calculated results are due to the statistical nature of the simulations: a non-infinite number of symbols need to be used to limit the simulation time.

The baseband part of the PSD (up to the Nyquist frequency $f_{N}$, which is at 0.5 on the $\mathrm{x}$-axis) is very similar to that of the SSF filter. As expected, the 2PWM scheme has a higher power spectral density at frequencies above the baseband than the 3PWM scheme, due to its higher switching frequency. This is a well-known side effect of PWM. As long as the channel has a low-pass transfer function, the high-frequency part of the spectrum will be filtered out by the channel. Also a low-pass filter inside the transmitter could be used to filter out those components. A system designer should weigh up this side-effect of PWM against the advantage (for high-speed low-voltage CMOS technologies) of using a transmission equalizer that only needs to switch between two voltages.

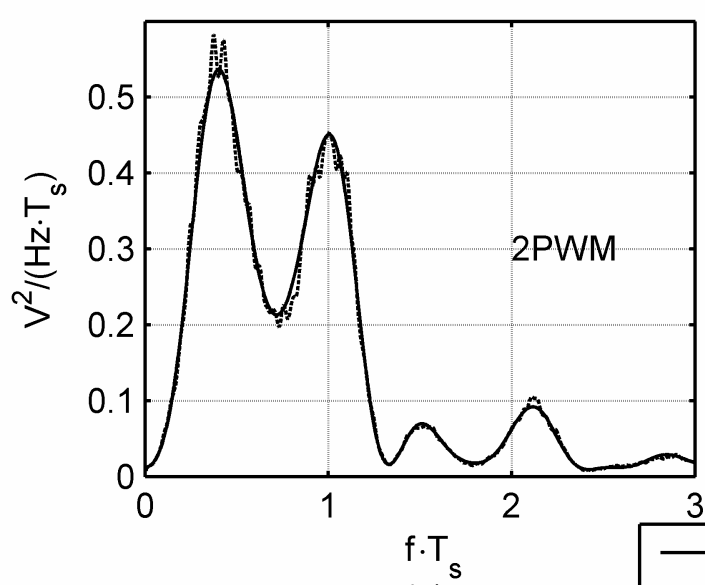

(a)

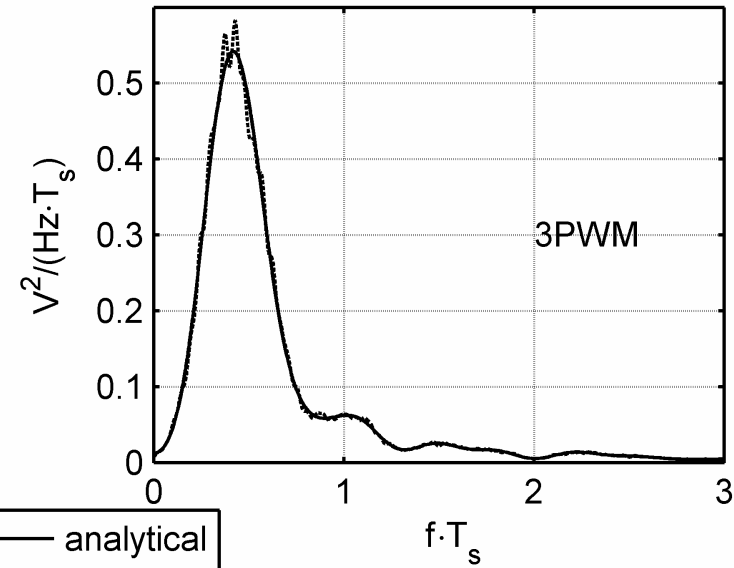

(b)

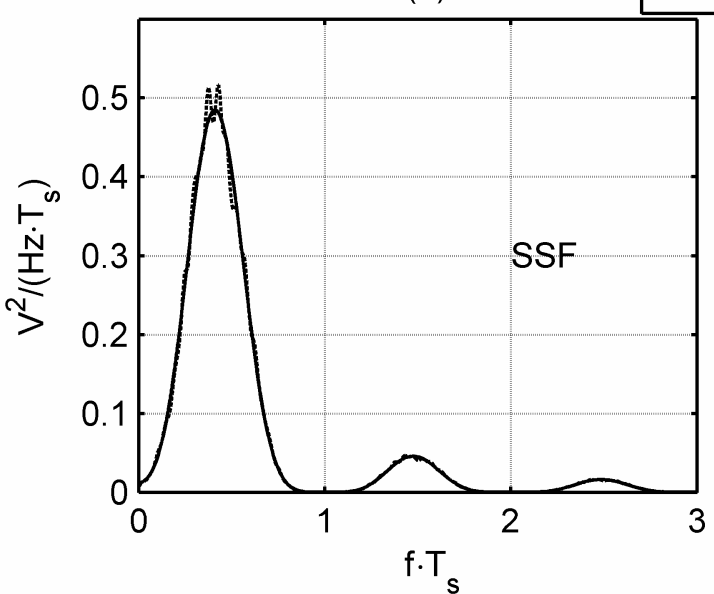

(c)

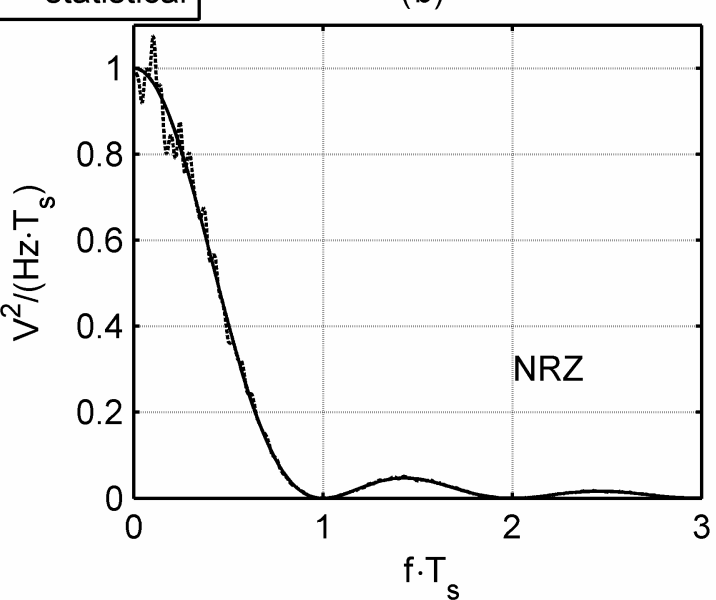

(d)

Fig. 12. PSDs (from analytical and from statistical simulations). Smooth lines are for 'analytical', jagged lines are for 'statistical'. (a) 2PWM (2 level, 3 tap). (b) 3PWM (3 level, 3 tap). (c) 3-tap SSF. (d) Polar NRZ. 


\subsection{Reducing the number of transitions in the 2PWM scheme}

A possible disadvantage of the 2PWM scheme, presented in the previous sections, is its large number of transitions - and the resulting large width of the PSD. The output voltage switches twice in each singlet. There might be unnecessary transitions in the scheme, adding power in higher frequencies that are outside of the signal band. We want to see whether the amount of transitions can be decreased by moving to another scheme. In this final section, we decrease the number of transitions per second of the PWM multitap filter by adjusting the 2PWM scheme that was presented above. The goal is to keep the eye opening the same, while making the PSD narrower.

We still have an extra degree of freedom with the 2PWM scheme. This degree of freedom is the placing of the pulse in the singlet: whether the pulse is placed in the middle of the bit time, or at the left or right side. Up until now, our PWM scheme placed the pulse in the middle. However, looking back at the 1-tap PWM pulse shape from Chapter 4, we see that this pulse first has a positive voltage, and then a negative voltage. So it is placed on the left side (which is the best for a channel having predominantly post-cursor ISI). There is only one transition inside the singlet instead of two in the 3PWM and 2PWM schemes. To reduce the number of transitions, we can change our 2PWM scheme. We choose to move the pulse to the left of the singlet. This new scheme is illustrated in Fig. 13, dubbed the 2PWM-L scheme (' $\mathrm{L}$ ' from 'left').

A: $-11-1$

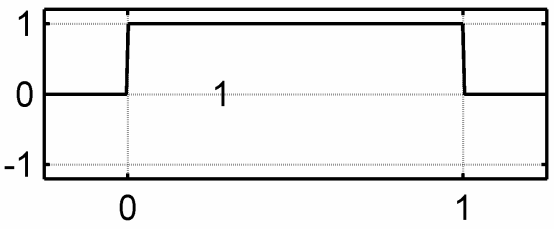

B: -111
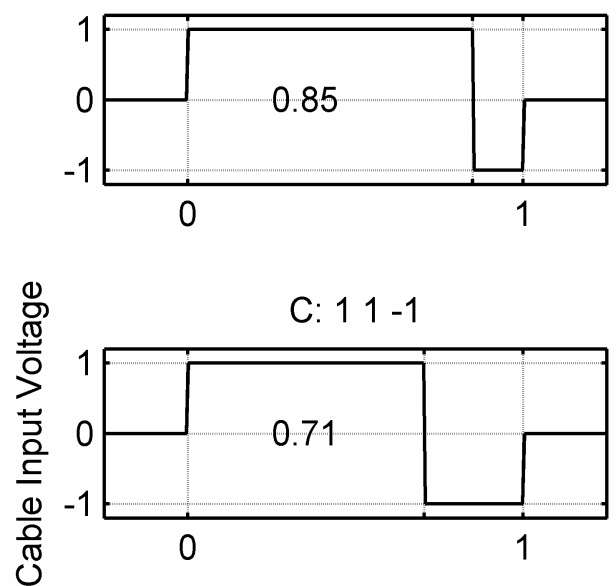

D: 111

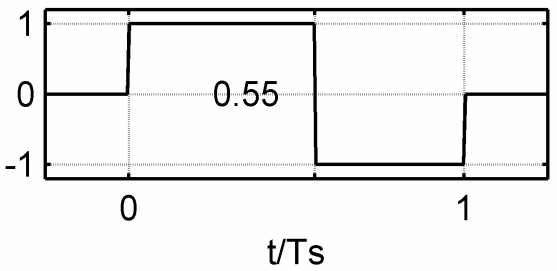

$-A: 1-11$

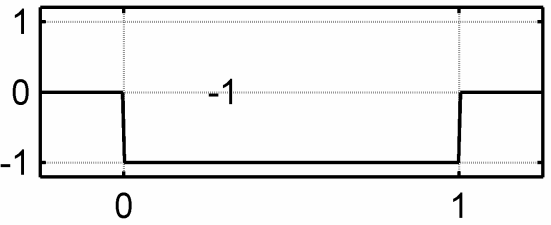

-B: $1-1-1$
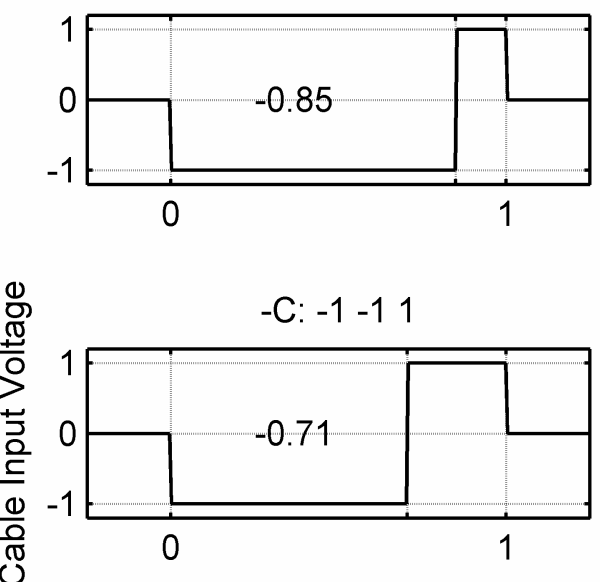

-D: $-1-1-1$

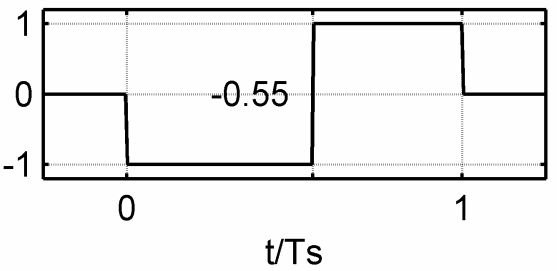

Fig. 13. Singlet shapes for 2PWM-L scheme. 
The 2PWM-L singlet is defined as follows:

$$
y_{2 P W M-L}(t)=\left\{\begin{array}{cc}
0, & t<0, \\
\operatorname{sign}(\psi), & t<|\psi| T_{s}, \\
-\operatorname{sign}(\psi), & t>|\psi| T_{s}, \\
0, & t>T_{s},
\end{array}\right.
$$

where, at first, the same parameter values for $\psi$ (denoting pulse-width and polarity) as in the 2PWM scheme are used.

Judging by the transient simulation and eye diagram in Fig. 14, we can easily see that the 2PWM-L scheme does not fit well to the channel. (The same channel is used as in the simulations with the previously discussed schemes.) Shifting the pulses to the left side of the singlets has changed the behavior of the filter in such a way that it is no longer enough to derive the pulse width simply from the SSF singlet height. The phase relations between the singlets are changed compared to the previous schemes, because the length of time that they are moved to the left depends on their width. We need to compensate for this by using different pulse-widths.

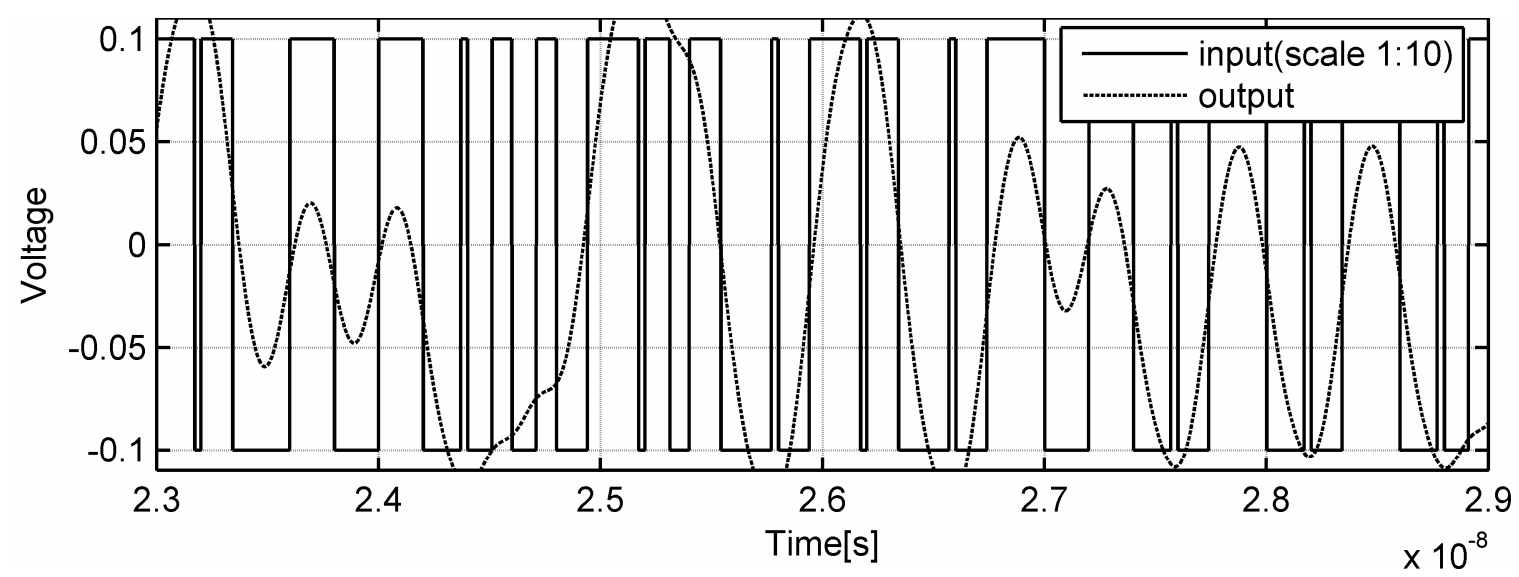

(a)

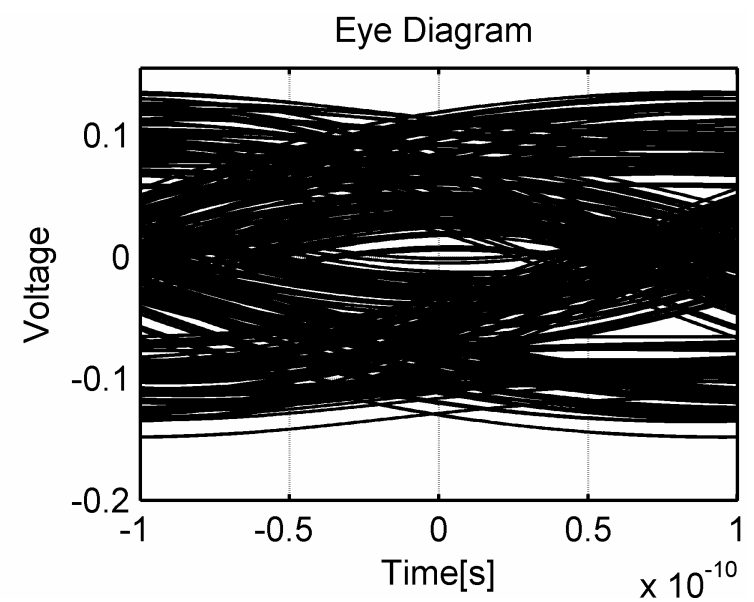

(b)

Fig. 14. Response of example channel to $2 \mathrm{PWM}-\mathrm{L}$ scheme, $\mathrm{T}_{\mathrm{s}}=200 \mathrm{ps}$. (a) Time domain.

(b) Eye diagram. 
After some trial-and-error simulation runs, it was discovered that swapping singlets $\mathrm{B} \& \mathrm{C}$ (and $-\mathrm{B} \&-\mathrm{C}$ ) provided a solution to the problem. This is equivalent to calculating the singlet pulse-widths from the SSF filter taps with taps 1 and 3 swapped. This new scheme incorporating the swap, is denoted as 2PWM-LBC. Mathematically, we define this scheme as follows. In Eq. 21, $\psi$ is replaced by $\psi_{\text {alt }}$, where $\psi_{\text {alt }}$ is given by:

$$
\left|\psi_{\text {alt }}\right|=\left(\left|\alpha_{\text {alt }}\right|+1\right) / 2,
$$

and $\alpha_{\text {alt }}$ is given by (compare Eq. 4):

$$
\alpha_{a l t}=w_{1} \cdot b_{n-1}+w_{2} \cdot b_{n}+w_{3} \cdot b_{n+1},
$$

where $b$ is the bit sequence, and $w$ represents the tap weight values. This scheme is illustrated in Fig. 15.

In Fig. 16(b) the simulated time domain response of the channel to the 2PWM-LBC scheme is shown. For comparison, the response to the 2PWM scheme is repeated in Fig 16(a). Indeed, an open eye is seen and the number of transitions is lower (at 43) compared to the 2PWM scheme (at 51), just as we desired. Fig 17 compares the eye diagrams. The goal of keeping the eye opening the same is achieved.

A: $-11-1$

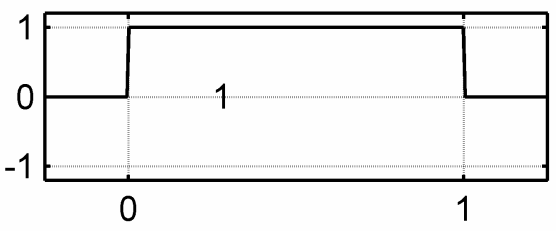

B: -111
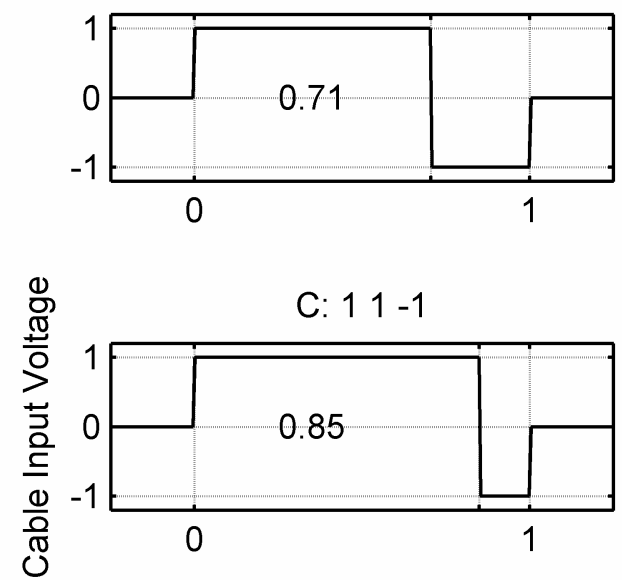

D: 111

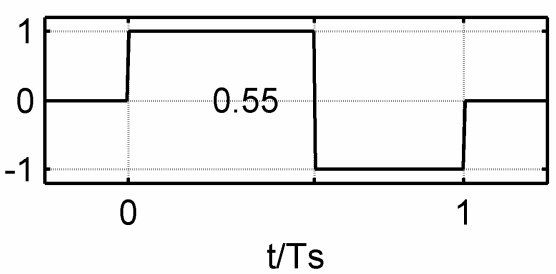

$-A: 1-11$

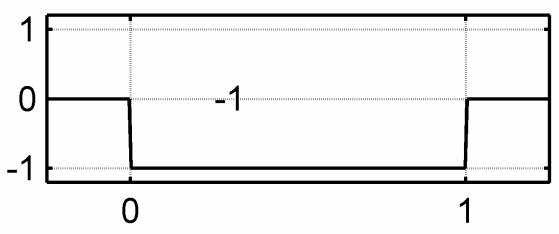

-B: $1-1-1$
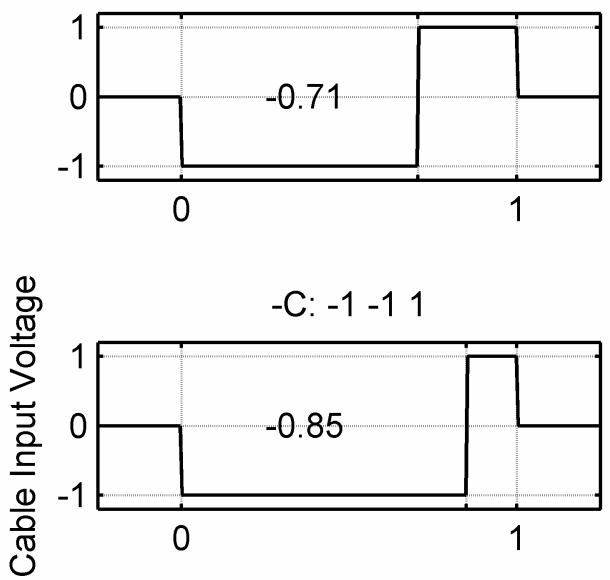

-D: $-1-1-1$

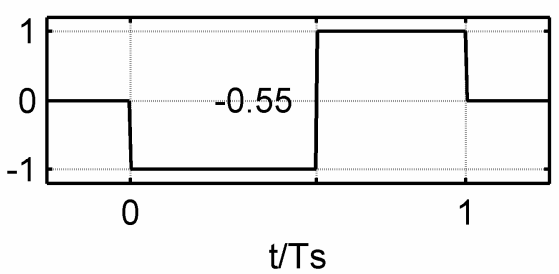

Fig. 15. Singlet shapes for the 2PWM-LBC scheme. 


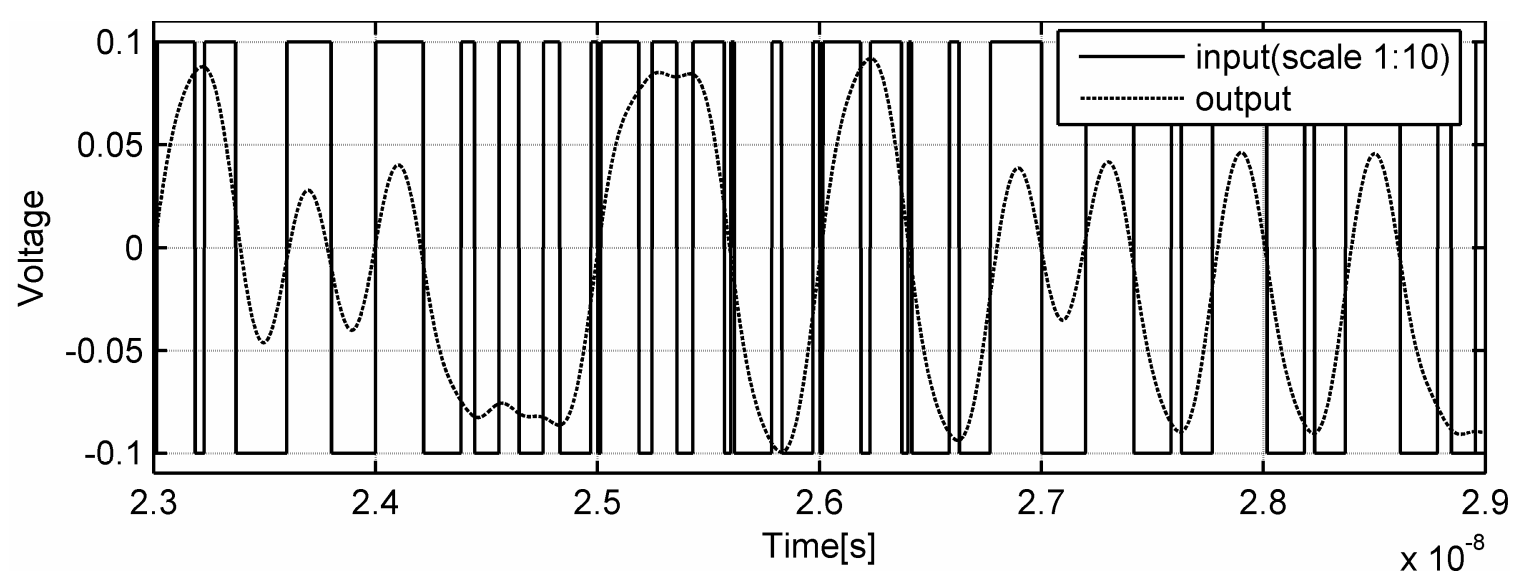

(a)

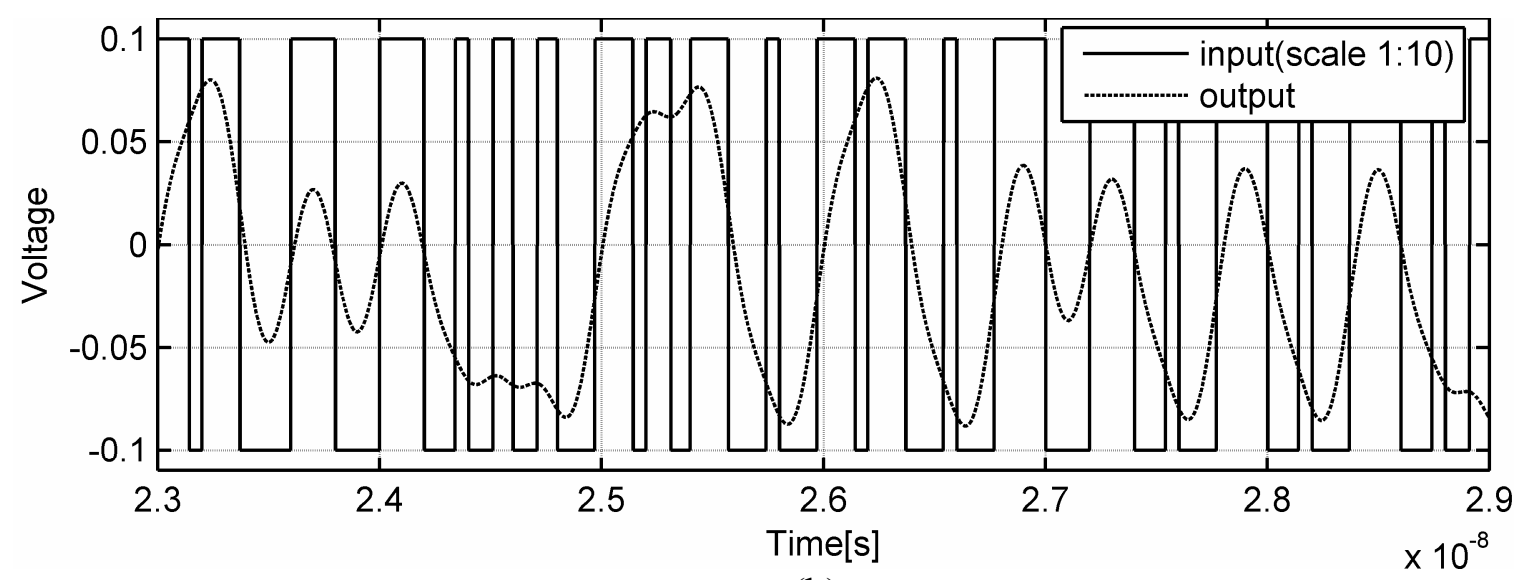

(b)

Fig. 16. Simulated transient response of example channel to 2PWM and to 2PWM-LBC, and transmitter outputs (=channel inputs), $\mathrm{T}_{\mathrm{s}}=200 \mathrm{ps}$. Both PWM filters have three taps and two output voltage levels. (a) 2PWM (51 transitions). (b) 2PWM-LBC (43 transitions).

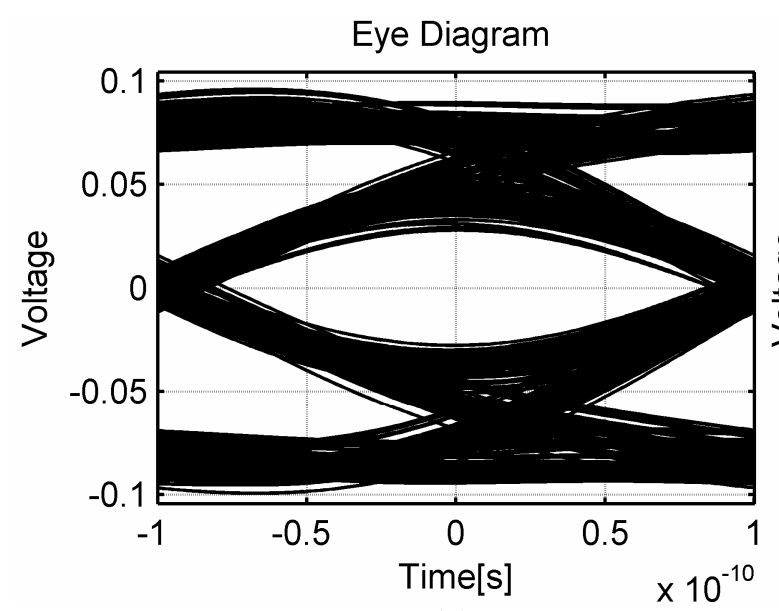

(a)

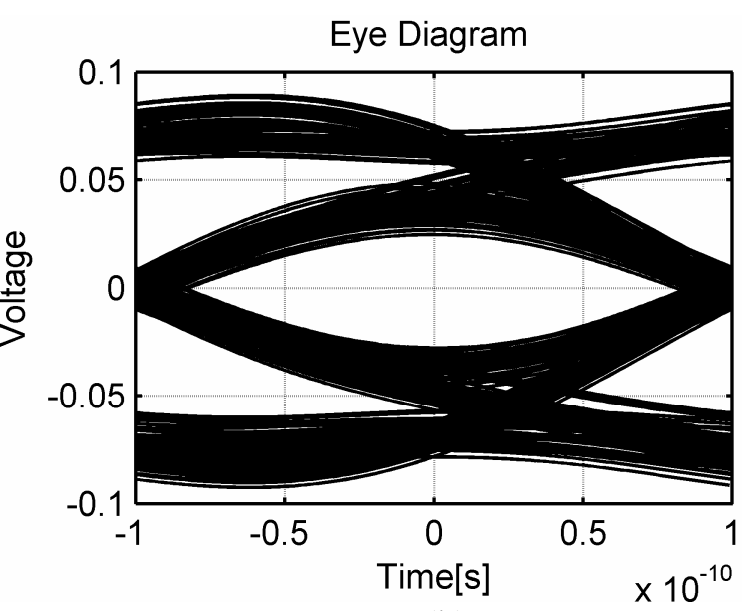

(b)

Fig. 17. Eye diagrams of response of example channel to 2PWM input and to 2PWM-LBC input, $\mathrm{T}_{\mathrm{s}}=200$ ps. (a) 2PWM. (b) 2PWM-LBC. 
Next, we would like to know whether the PSD of the 2PWM-LBC scheme is narrower than that of the 2PWM scheme. Fig. 18 shows the autocorrelations and PSDs of the 2PWM-L and 2PWM-LBC schemes described above. In Fig. 19, the PSDs are compared to those of the 3tap SSF and the 2PWM scheme. In Fig. 19, we can clearly see that the PSD for 2PWM-L is indeed very different from the others (in the baseband). The PSD for the 2PWM-LBC scheme (with B\&C swapped) again matches the PSD of 3-tap SSF more closely. The second lobe for the 2PWM-LBC scheme has moved to the left a little bit (the PSD is narrower) compared to the 2PWM scheme, indicating that the switching frequency is lower. However, the power spectral density around $0.7 \mathrm{~Hz} \cdot T_{S}$ has increased.

As an aside, we note that the PWM scheme described in Chapter 4 is in fact a subset of the 2PWM-L and 2PWM-LBC schemes. We can obtain the previous PWM scheme by making the pulse-widths for singlets $\{\mathrm{A}, \mathrm{B}, \mathrm{C}, \mathrm{D}\}$ in the $2 \mathrm{PWM}-\mathrm{L}$ scheme equal to $\{d, d, d, d\}$, where $d$ is the duty-cycle of the PWM scheme from Chapter 4.

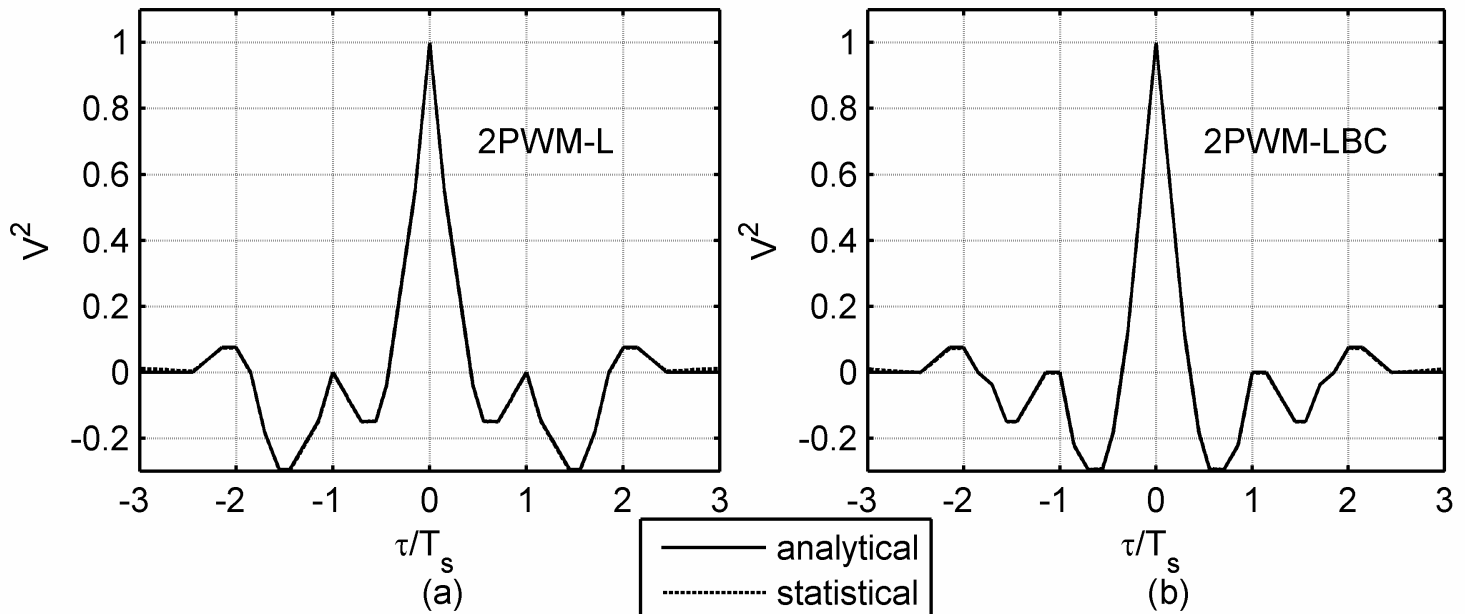

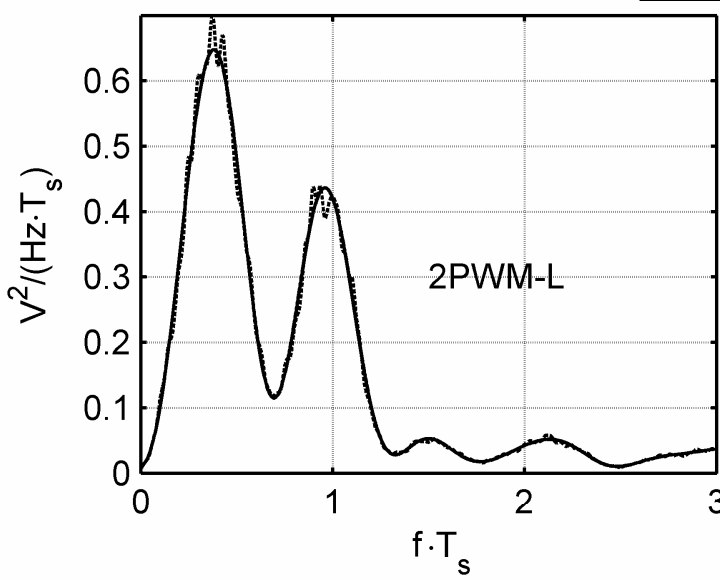

(c)

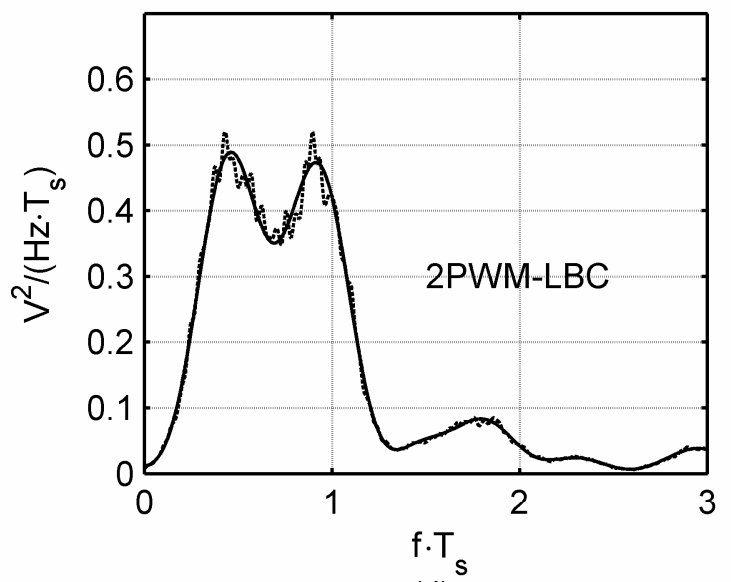

(d)

Fig. 18. Autocorrelations and PSDs for 2PWM-L and 2PWM-LBC schemes (from analytical and from statistical simulations). In some plots only the 'analytical' line can be seen because the 'statistical' line is right below it. (a) Autocorrelation for 2PWM-L. (b) Autocorrelation for 2PWM-LBC. (c) PSD for 2PWM-L. (d) PSD for 2PWM -LBC. 


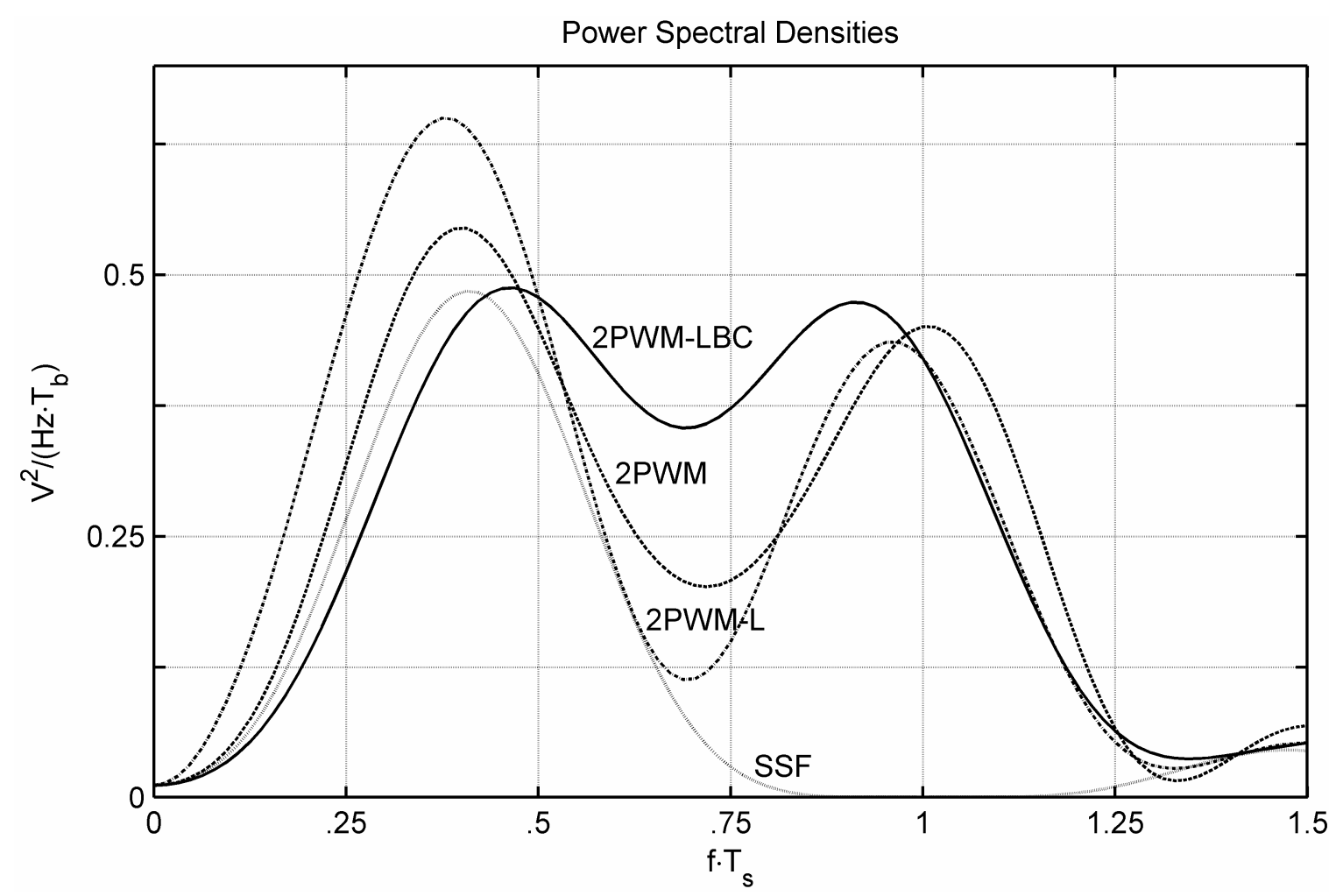

Fig. 19. PSDs for 3-tap SSF, 2PWM, 2PWM-L and 2PWM-LBC schemes.

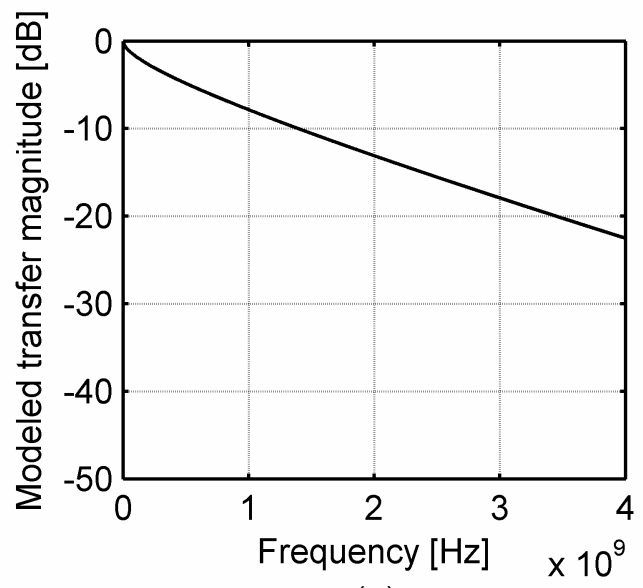

(a)

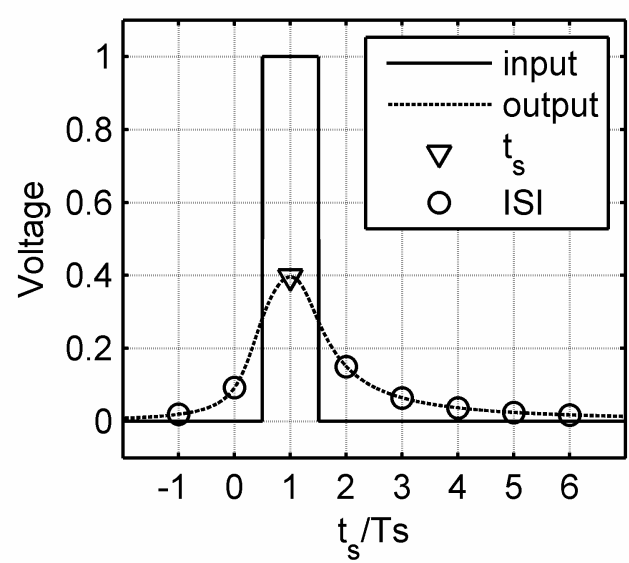

(b)

Fig. 20. Alternative (theoretical) channel used for time domain simulations. (a) Channel loss.

(b) pulse response $\left(T_{s}=200 \mathrm{ps}\right)$.

The question may arise of whether the 2PWM-LBC scheme also works for channels with lower loss levels. To evaluate this, time domain simulations were done with an alternative channel. The loss and pulse response for this channel are shown in Fig. 20. The channel has approximately $15 \mathrm{~dB}$ loss at the Nyquist frequency of $2.5 \mathrm{GHz}$. (The bit rate is again chosen to be $5 \mathrm{~Gb} / \mathrm{s}$.)

In Fig. 21, the eye diagrams of the response of this alternative channel to the 2PWM-L scheme (Fig. 21(a)) and to the 2PWM-LBC scheme (Fig. 21(b)) are shown. Clearly, the channel response to the 2PWM-LBC scheme shows a larger eye width, indicating that for this alternative channel, the phase relations between the singlets in the 2PWM-LBC scheme are also a better fit than those in the 2PWM-L scheme. 


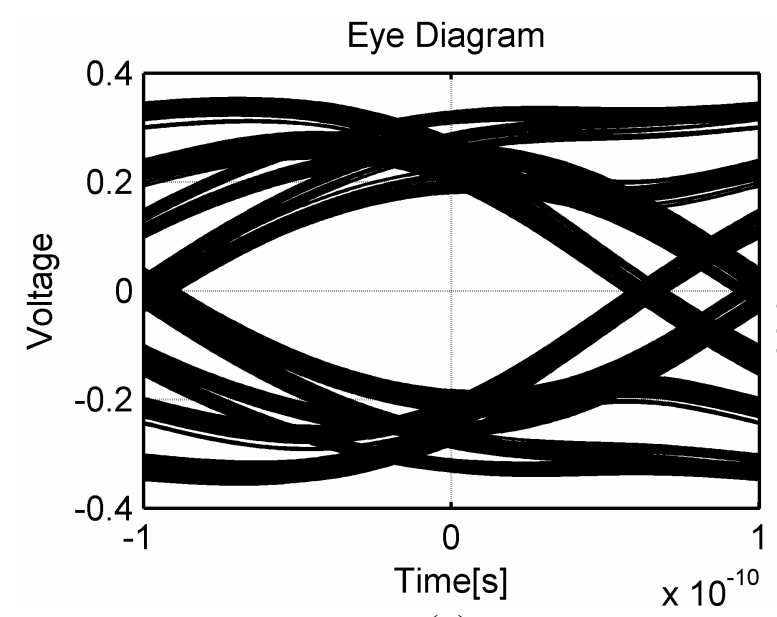

(a)

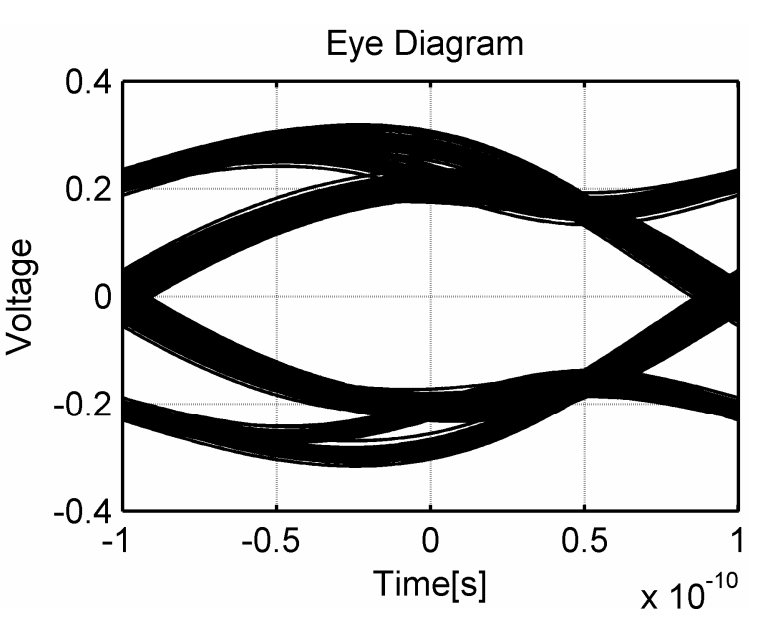

(b)

Fig. 21. Eye diagrams of response of alternative channel to 2PWM-L input and to 2PWMLBC input, $T_{s}=200 p s$. (a) 2 PWM-L. (b) 2PWM-LBC.

In conclusion, we have found an alternative 3-tap, 2-voltage-level PWM scheme that a gives a comparable eye opening as the 2PWM scheme while making less transitions per second. The pulse-widths were found by calculating them from the SSF filter taps with taps 1 and 3 swapped.

\subsection{Conclusions}

We can extend the PWM pre-emphasis technique to a multitap version. The duty-cycle of this multitap PWM filter is a function of multiple bits instead of a function of only the current bit, as in Chapter 4. In this way, more complex filter transfer functions can be constructed, as with multitap FIR filters. We can use this for example to cancel reflections on backplane PCBs. The transmitter needs to switch between only two voltages, again allowing a transmitter implementation that is based on timing accuracy instead of on amplitude accuracy. The correct switch timing needs to be found as a function of multiple bits, in order to create the desired (effective) filter transfer function. This can be done by deriving the pulse-widths from the SSF filter taps by using a 'same-area' approach.

Three 3-tap PWM filters were discussed:

1) ' $3 \mathrm{PWM}$ ': three output voltage levels $(-1 \mathrm{~V}, 0 \mathrm{~V}$ and $1 \mathrm{~V})$,

2) '2PWM': two output voltage levels (-1V and $1 \mathrm{~V})$,

3) '2PWM-LBC': two output voltage levels (-1V and $1 \mathrm{~V})$ and a reduced number of transitions.

The ' $3 P W M$ ' filter transmits, during the time length of one bit, first $0 \mathrm{~V}$, then $1 \mathrm{~V}$, then $0 \mathrm{~V}$ again. The '2PWM' filter transmits, during the time length of one bit, first $-1 \mathrm{~V}$, then $1 \mathrm{~V}$ and then $-1 \mathrm{~V}$ again. The '2PWM-LBC' filter transmits first $1 \mathrm{~V}$, then $-1 \mathrm{~V}$ (less transitions). The voltage levels are inverted when the equivalent FIR filter would otherwise output a negative voltage.

Time domain simulations show eye openings nearly equal to those of the equivalent 3-tap SSF filter. 
The power spectral density functions of the 3-tap PWM filters are calculated using their autocorrelation functions. At frequencies around the Nyquist frequency, these filters have a higher power spectral density than the SSF filter due to harmonic signals, inherent to PWM.

A system designer should weigh up this side-effect of PWM against the abovementioned advantage of using a transmission equalizer that needs to switch between only two voltages. As long as the channel has a low-pass transfer function, the high-frequency part of the spectrum will be filtered out by the channel.

The '2PWM-LBC' filter reduces the number of transitions compared to the '2PWM' scheme. This reduces the HF energy transmitted, since the PSD is narrowed. However, shifting the pulses to the left disturbs their phase relations. Using transient simulations, it was found that the receiver eye diagram can be opened up again by using pulse-widths calculated from the SSF filter taps with taps 1 and 3 swapped. The resulting eye opening is nearly the same as for the '2PWM' scheme. 


\section{Chapter 6}

\section{Conclusions}

In this chapter, the thesis is summarized (section 6.1), the original contributions are listed (section 6.2), and finally suggestions for future research are given in section 6.3.

\subsection{Summary of the thesis}

First, the main research questions are repeated in subsection 6.1.1. Following that, a perchapter summary is given.

\subsubsection{Main research questions}

Copper channels suffer from attenuation and dispersion, caused by the skin effect and dielectric loss. Furthermore, they suffer from reflections, caused by impedance discontinuities. As a consequence, the achievable bit rate is limited by intersymbol interference: neighboring symbols interfere with each other.

The aim of this research was to find new equalization and modulation techniques that can be used to increase the data rate over copper interconnects while maintaining an acceptable bit error rate. These techniques must be compatible with future CMOS generations.

\subsubsection{Chapter 2}

Chapter two describes a practical model for copper cables and PCBs, for use in transient simulations. The model, incorporating both the skin effect and dielectric loss, gives a causal impulse response because the complex skin and dielectric impedances both comply with the Kramers-Kronig relations.

The model is fitted to coaxial and twisted pair cables and to a PCB. Model parameters for these channels are given.

\subsubsection{Chapter 3}

An analog OFDM system - based on analog mixers and integrate-and-dump blocks - is presented and analyzed. The impact of timing non-idealities such as jitter and duty-cycle deviations is analyzed.

The main findings were as follows.

- $\quad$ Jitter causes crosstalk between the in-phase and quadrature channels. Given a certain RMS jitter variance, low frequency tones can carry more bits than higher frequency tones for the same error rate, because the same time shift gives a different phase error at different frequencies.

- Duty-cycle deviations cause crosstalk between carriers at different frequencies because the OFDM orthogonality is lost. 
- Harmonics from the switching mixers hinder the use of a large number of subcarriers, because of spectral overlap.

It is concluded that, because of the high sensitivity to timing errors, this analog OFDM system is currently infeasible and traditional PAM systems are more feasible (and more straightforward to implement) for copper channels with monotonously decreasing transfer functions. For copper channels with nulls in the transfer function and for copper channels that suffer from ingress noise, OFDM offers important advantages (for example in ADSL).

\subsubsection{Chapter 4}

A transmit equalizer for copper cables and PCBs can be made by having a transmitter switch between only two voltages. During the time length of one bit, if a ' 1 ' bit needs to be transmitted, first a positive voltage is transmitted and then a negative voltage, and vice versa if a ' 0 ' bit needs to be transmitted. By adjusting the ratio of the duration of these two voltages (the duty-cycle), the filter can be adjusted to the channel. This PWM filter thus has only one 'knob' (adjustment parameter).

This technique provides an alternative to the usual symbol-spaced FIR (SSF) pre-emphasis filters. The technique relies on timing resolution instead of on amplitude resolution, as the FIR filters do. This can be advantageous in the light of CMOS scaling - with ever increasing switching speeds and decreasing voltage headroom.

The transfer function of the PWM filter happens to fit very well to copper channels. The PWM filter more closely matches copper channels than both a 2-tap SSF filter and a 2-tap half-symbol spaced FIR filter. At the Nyquist frequency, the calculated magnitude of the PWM filter transfer function has a value of one while its derivative is non-zero, unlike with the 2-tap SSF filter. The PWM filter transfer function can be seen as a higher order filter with only one adjustment parameter. Time domain simulations with a skin-effect-only channel show that approximately twice the bit rate can be achieved compared to a 2-tap SSF for the same peak distortion.

A normal pulse amplitude modulated (PAM) signal is received at the end of the channel. Analysis shows that the eye opening at the receiver is unchanged compared to a 2-tap symbolspaced FIR (SSF) filter (assuming that they both have the same voltage headroom). Therefore, the receiver circuits can remain unchanged.

Two $0.13 \mu \mathrm{m}$ CMOS chips and one 90nm CMOS chip were made as a proof-of-concept.

Measurements with these prototype chips show successful reception of data transmitted over coaxial and twisted pair copper cables, with up to $30 \mathrm{~dB}$ loss at the Nyquist frequency, with BER $10^{-12}$, compared to $20 \mathrm{~dB}$ for a 2 -tap SSF filter (and $30 \mathrm{~dB}$ for more complex equalizers with more adaptation parameters). A bit rate of $5 \mathrm{~Gb} / \mathrm{s}$ was achieved over all channels. The cable loss consisted of different combinations of skin effect and dielectric loss. On an FR4 microstrip PCB trace, $25 \mathrm{~dB}$ loss compensation was measured.

\subsubsection{Chapter 5}

We can extend the PWM pre-emphasis technique to a multitap version. The duty-cycle of this multitap PWM filter is a function of multiple bits instead of a function of only the current bit, 
as in Chapter 4. In this way, more complex filter transfer functions can be constructed, as with multitap FIR filters. The transmitter needs to switch between only two voltages, again allowing a transmitter implementation that is based on timing accuracy instead of on amplitude accuracy. The correct switch timing needs to be found as a function of multiple bits, in order to create the desired (effective) filter transfer function. This can be done by deriving the pulse-widths from the SSF filter taps by using a 'same-area' approach. Time domain simulations show eye openings nearly equal to those of the equivalent 3-tap SSF filter.

The power spectral density functions of the 3-tap PWM filters are calculated using their autocorrelation functions. At frequencies around the Nyquist frequency, these filters have a higher power spectral density than the SSF filter due to harmonic signals, inherent to PWM. A system designer should weigh up this side-effect of PWM against the abovementioned advantage of using a transmission equalizer that needs to switch between only two voltages. As long as the channel has a low-pass transfer function, the high-frequency part of the spectrum will be filtered out by the channel.

\subsection{Original contributions}

The discovery that by applying pulse-width modulation (PWM) pre-emphasis to wireline equalization, $30 \mathrm{~dB}$ of channel loss at the Nyquist frequency $f_{N}$ can be overcome (with BER $<10^{-12}$ ), versus only $20 \mathrm{~dB}$ with the commonly used 2-tap symbol-spaced FIR (SSF) pre-emphasis filter.

- $\quad$ The discovery that $30 \mathrm{~dB}$ loss compensation can be achieved using a PWM equalizer with only a single adjustment parameter, namely the duty-cycle.

Time-domain simulations which show that the PWM filter achieves approximately twice the bit rate of a 2-tap SSF for the same peak distortion on a theoretical skineffect-only channel.

- $\quad$ Calculations which show that the eye height at the receiver is the same, whether PWM pre-emphasis or 2-tap SSF is used, because both filters have a transfer function of one at $f_{N}$.

A comparison of the transfer functions of the PWM pre-emphasis filter and of the 2-tap SSF filter. At the Nyquist frequency, the calculated magnitude of the PWM filter transfer function has a value of 1 while its derivative is non-zero, unlike with the 2-tap SSF filter.

Realizations of the PWM pre-emphasis transmitter in both $0.13 \mu \mathrm{m}$ and $90 \mathrm{~nm}$ CMOS chips.

Several multitap PWM schemes - especially one scheme with a reduced number of transitions - for a 3-tap PWM filter. Such multitap PWM filters can provide more complex transfer functions, as with a multitap FIR filter.

Calculations which show that in an 'analog' OFDM system, jitter and duty-cycle variations cause crosstalk between the in-phase and quadrature channels at a given frequency, and between subcarriers at different frequencies, both of which limit the achievable bit rate. 


\subsection{Recommendations for future research}

A more generalized mathematical analysis should be made of the multitap PWM filter. More links should be made to existing PWM theory and schemes.

Using a multitap PWM filter, it is possible to construct complex filter transfer functions. We should think of applications such as canceling reflections on PCBs. The multitap PWM filters should be implemented and tested.

Low jitter is essential to achieve high loss compensation with PWM pre-emphasis. Therefore, it is important to further explore low-jitter PWM circuits, as this directly impacts on the performance of the PWM pre-emphasis transmitter.

- $\quad$ It is possible to combine PWM pre-emphasis with multilevel PAM systems to improve the spectral efficiency (if the SNR is sufficiently high). 


\section{Appendix A}

\section{Diffusion equation for skin effect}

As an interesting aside, related to the 'Nanowire' $\mathrm{PhD}$ project in our group researching onchip communication, we note that the response of the skin-effect-only copper channel, and a characteristically terminated on-chip RC-limited wire are the same. In both cases, a diffusion equation can be used to describe the channel [Ramo], [Kerst]. For the skin effect in a cylindrical conductor, the magnetic field is written in the diffusion equation. In an on-chip RC-limited wire, the voltage is written in the diffusion equation (charge diffusion). More detail is given below.

In an RC-limited wire, the diffusion equation is [Dally-3]:

$$
\frac{\partial^{2} V}{\partial x^{2}}=R C \frac{\partial V}{\partial t}
$$

For the skin effect, the diffusion equation is [Kerst]:

$$
\frac{\partial^{2} B}{\partial x^{2}}=\sigma \mu \frac{\partial B}{\partial t}
$$

where $B$ is the magnetic field. For a cylindrical conductor with an axial current, this equation becomes [Kerst]:

$$
\frac{\partial B_{\theta}}{\partial t}=\frac{1}{\mu \sigma}\left(\frac{\partial^{2} B_{\theta}}{\partial r^{2}}+\frac{1}{r} \frac{\partial B_{\theta}}{\partial r}-\frac{B_{\theta}}{r^{2}}\right),
$$

with $r$ the direction of the radius and where $B_{\theta}$ is the azimuthal magnetic field.

The diffusion process in the case of the skin effect in a cylindrical conductor is that of the magnetic field into the conductor. At time $t=0$, the magnetic field is only present on the outer edge of the conductor, whilst at $t=\infty$ the field has penetrated the entire conductor. In the intervening time, a diffusion process takes place in the radial direction. 


\section{Appendix B}

\section{Jitter during the integration period}

Jitter during the integration period $T_{c}$ will effectively lead to duty cycle distortion, which causes inter-carrier interference. We have to make sure that this effect does not invalidate the analysis in Chapter 3. From [McNeill], the jitter variance as a function of measurement time is:

$$
\sigma_{t}=\kappa \sqrt{\tau}
$$

where $\kappa$ is a time domain figure of merit. When we fill in $T_{c}$ for $\tau$ in Eq. 1 and divide by $T_{c}$, we obtain the following formula:

$$
\frac{\sigma_{t}}{T_{c}}=\frac{\kappa}{\sqrt{T_{c}}}
$$

To provide a state-of-the-art estimation for $\kappa$, we use the following formulas from [McNeill]:

$$
\begin{gathered}
S_{\phi}(f)=\frac{N}{f^{2}}, \\
\kappa=\frac{\sqrt{N}}{f_{0}} .
\end{gathered}
$$

Using a phase noise of $-99.5 \mathrm{dBc} / \mathrm{Hz}$ at $1 \mathrm{MHz}$ offset for the ("state-of-the-art") three-stage ring oscillator in [Eken] we calculate its $\kappa \approx 2 \cdot 10^{-9} \sqrt{\mathrm{s}}_{\mathrm{s}}$. By substituting this value into Eq. 2 and assuming that $T_{c}>1 \cdot 10^{-10} \mathrm{~s}$, we can calculate that:

$$
\frac{\sigma_{t}}{T_{c}}<2 \cdot 10^{-4}
$$

In order for the correlation plot to be used for calculating bit error rates in the order of $1 \cdot 10^{-12}$, we calculate the $7 \sigma$ variation to be approximately $1 / 1000^{\text {th }}$ of a period, having little or no effect on carrier orthogonality. 


\section{Appendix C}

\section{Tables for autocorrelation calculations}

As explained in Chapter 5, the autocorrelation function $R_{x x}(\tau)$ is calculated by summing three components $R_{0}(\tau), R_{1}(\tau)$ and $R_{2}(\tau)$ as follows:

$$
R_{x x}(\tau)=R_{2}\left(\tau+2 T_{s}\right)+R_{1}\left(\tau+T_{s}\right)+R_{0}(\tau)+R_{1}\left(-\tau-T_{s}\right)+R_{2}\left(-\tau-2 T_{s}\right) .
$$

The tables used for calculating these three components, showing the possible combinations of singlets $v_{k}(i, j)$ and their probability, are given below. First the table for $R_{0}(\tau)$ is given, next for $R_{l}(\tau)$ and finally for $R_{2}(\tau)$.

The following property of the cross-correlation is used in the calculations:

$$
R_{x,-y}(\tau)=R_{-x, y}(\tau)=-R_{x, y}(\tau)
$$

A shorthand notation is used when writing down the correlations, e.g. $R_{A B}$ means:

$$
R_{A B}=\int_{0}^{T_{s}} y_{1}(t) y_{2}(t+\tau) d t,
$$

where $y_{1}(t)$ is the singlet ' $\mathrm{A}$ ' and $y_{2}(t)$ is the singlet ' $\mathrm{B}$ ' (see Table 1 in Chapter 5).

\section{C.1. $R_{0}(\tau)$}

\begin{tabular}{|r|rrr|l|l|l|}
\hline \multicolumn{3}{|l|}{ Singlet } & $v_{0}(i, j)$ & Probability & Term \\
\hline A & -1 & 1 & -1 & $v_{0}(1,1)$ & $1 / 8$ & AA \\
\hline -A & 1 & -1 & 1 & $v_{0}(2,1)$ & $1 / 8$ & AA \\
\hline B & -1 & 1 & 1 & $v_{0}(3,1)$ & $1 / 8$ & BB \\
\hline - B & 1 & -1 & -1 & $v_{0}(4,1)$ & $1 / 8$ & BB \\
\hline C & 1 & 1 & -1 & $v_{0}(5,1)$ & $1 / 8$ & CC \\
\hline -C & -1 & -1 & 1 & $v_{0}(6,1)$ & $1 / 8$ & CC \\
\hline D & 1 & 1 & 1 & $v_{0}(7,1)$ & $1 / 8$ & DD \\
\hline$-D$ & -1 & -1 & -1 & $v_{0}(8,1)$ & $1 / 8$ & DD \\
\hline \multicolumn{8}{|c|}{ Table $1 . R_{0}(\tau)}$. &
\end{tabular}

Adding up the terms in the above table, we obtain $R_{0}(\tau)$ :

$$
\begin{aligned}
& R_{0}(\tau)=\frac{1}{8}\left(R_{A A}+R_{A A}+R_{B B}+R_{B B}+R_{C C}+R_{C C}+R_{D D}+R_{D D}\right) \\
& =\frac{1}{4}\left(R_{A A}+R_{B B}+R_{C C}+R_{D D}\right) .
\end{aligned}
$$


C.2. $R_{1}(\tau)$

\begin{tabular}{|c|c|c|c|c|c|c|c|}
\hline \multicolumn{2}{|c|}{ Singlet 1} & \multicolumn{2}{|c|}{ Singlet 2} & $v_{1}(i, j)$ & \multicolumn{2}{|c|}{ Probability } & Term \\
\hline \multirow[t]{8}{*}{$\mathrm{A}$} & \multirow{8}{*}{$\begin{array}{lll}-1 & \mathbf{1} & -\mathbf{1}\end{array}$} & $\mathrm{A}$ & $\begin{array}{lll}-1 & 1 & -1\end{array}$ & & & - & \\
\hline & & $-\mathrm{A}$ & $\begin{array}{lll}1 & -1 & 1 \\
\end{array}$ & $v_{1}(1,1)$ & $1 / 8 \cdot 1 / 2$ & $1 / 16$ & $A \cdot-A$ \\
\hline & & $\mathrm{B}$ & $\begin{array}{lll}-1 & 1 & 1\end{array}$ & & & - & \\
\hline & & $-\mathrm{B}$ & $\begin{array}{lll}\mathbf{1} & \mathbf{- 1} & -1 \\
\end{array}$ & $v_{1}(1,2)$ & $1 / 8 \cdot 1 / 2$ & $1 / 16$ & $A \cdot-B$ \\
\hline & & $\mathrm{C}$ & $\begin{array}{lll}1 & 1 & -1\end{array}$ & & & - & \\
\hline & & $-\mathrm{C}$ & $\begin{array}{lll}-1 & -1 & 1\end{array}$ & & & - & \\
\hline & & $\mathrm{D}$ & $\begin{array}{lll}1 & 1 & 1 \\
\end{array}$ & & & - & \\
\hline & & $-\mathrm{D}$ & $\begin{array}{lll}-1 & -1 & -1\end{array}$ & & & - & \\
\hline & & & & & \multicolumn{3}{|r|}{$-1 / 16(\mathrm{AA}+\mathrm{AB})$} \\
\hline \multirow[t]{8}{*}{$\mathrm{B}$} & \multirow[t]{8}{*}{$\begin{array}{lll}-1 & 1 & 1\end{array}$} & $\mathrm{~A}$ & $\begin{array}{lll}-1 & 1 & -1 \\
\end{array}$ & & & - & \\
\hline & & $-\mathrm{A}$ & $\begin{array}{lll}1 & -1 & 1 \\
\end{array}$ & & & - & \\
\hline & & $\mathrm{B}$ & $\begin{array}{lll}-1 & 1 & 1 \\
\end{array}$ & & & - & \\
\hline & & $-B$ & $\begin{array}{lll} & -1 & -1\end{array}$ & & & - & \\
\hline & & $\mathrm{C}$ & $\begin{array}{lll}1 & 1 & -1 \\
\end{array}$ & $v_{1}(2,1)$ & $1 / 8 \cdot 1 / 2$ & $1 / 16$ & $B \cdot C$ \\
\hline & & $-\mathrm{C}$ & $\begin{array}{lll}-1 & -1 & 1 \\
\end{array}$ & & & - & \\
\hline & & $\mathrm{D}$ & $\begin{array}{lll}1 & 1 & 1 \\
\end{array}$ & $v_{1}(2,2)$ & $1 / 8 \cdot 1 / 2$ & $1 / 16$ & $B \cdot D$ \\
\hline & & $-\mathrm{D}$ & $\begin{array}{lll}-1 & -1 & -1 \\
\end{array}$ & & & - & \\
\hline & & & & & \multicolumn{3}{|r|}{$1 / 16(\mathrm{BC}+\mathrm{BD})$} \\
\hline \multirow[t]{8}{*}{$\mathrm{C}$} & \multirow[t]{8}{*}{$\begin{array}{lll}1 & 1 & -1\end{array}$} & $\mathrm{~A}$ & $\begin{array}{lll}-1 & 1 & -1 \\
\end{array}$ & & & - & \\
\hline & & $-\mathrm{A}$ & $\begin{array}{lll}1 & -1 & 1 \\
\end{array}$ & $v_{1}(3,1)$ & $1 / 8 \cdot 1 / 2$ & $1 / 16$ & $\mathrm{C} \cdot-\mathrm{A}$ \\
\hline & & $\mathrm{B}$ & $\begin{array}{lll}-1 & 1 & 1 \\
\end{array}$ & & & - & \\
\hline & & $-\mathrm{B}$ & $\begin{array}{lll}1 & -1 & -1 \\
\end{array}$ & $v_{l}(3,2)$ & $1 / 8 \cdot 1 / 2$ & $1 / 16$ & $\mathrm{C} \cdot-\mathrm{B}$ \\
\hline & & $\mathrm{C}$ & $\begin{array}{lll}1 & 1 & -1 \\
\end{array}$ & & & - & \\
\hline & & $-\mathrm{C}$ & $\begin{array}{lll}-1 & -1 & 1 \\
\end{array}$ & & & - & \\
\hline & & $\mathrm{D}$ & $\begin{array}{lll}1 & 1 & 1 \\
\end{array}$ & & & - & \\
\hline & & $-\mathrm{D}$ & $\begin{array}{lll}-1 & -1 & -1 \\
\end{array}$ & & & - & \\
\hline & $\begin{array}{lll} & & \\
1 & 1 & 1\end{array}$ & & & & \\
\hline \multirow[t]{8}{*}{$\mathrm{D}$} & \multirow{8}{*}{$\begin{array}{lll}1 & 1 & 1 \\
\end{array}$} & $\mathrm{~A}$ & $\begin{array}{lll}-1 & 1 & -1 \\
\end{array}$ & & $-1 / 10(C A+C B)$ & - & \\
\hline & & $-\mathrm{A}$ & $\begin{array}{lll}1 & -1 & 1 \\
\end{array}$ & & & - & \\
\hline & & $\mathrm{B}$ & $\begin{array}{lll}-1 & 1 & 1 \\
\end{array}$ & & & - & \\
\hline & & $-\mathrm{B}$ & $\begin{array}{lll}1 & -1 & -1 \\
\end{array}$ & & & - & \\
\hline & & $\mathrm{C}$ & $\begin{array}{lll}1 & 1 & -1 \\
\end{array}$ & $v_{1}(4,1)$ & $1 / 8 \cdot 1 / 2$ & $1 / 16$ & $\mathrm{D} \cdot \mathrm{C}$ \\
\hline & & $-\mathrm{C}$ & $\begin{array}{lll}-1 & -1 & 1 \\
\end{array}$ & & & - & \\
\hline & & $\mathrm{D}$ & $\begin{array}{lll}1 & 1 & 1 \\
\end{array}$ & $v_{l}(4,2)$ & $1 / 8 \cdot 1 / 2$ & $1 / 16$ & $\mathrm{D} \cdot \mathrm{D}$ \\
\hline & & $-\mathrm{D}$ & $\begin{array}{lll}-1 & -1 & -1 \\
\end{array}$ & & & - & \\
\hline & & & & & & & $1 / 16(\mathrm{DC}+\mathrm{DD})$ \\
\hline
\end{tabular}




\begin{tabular}{|c|c|c|c|c|c|c|c|c|}
\hline \multirow[t]{8}{*}{$-A$} & \multirow[t]{8}{*}{$\begin{array}{lll}1 & -1 & 1\end{array}$} & A & -1 & $1 \quad-1$ & $v_{1}(5,1)$ & $1 / 8 \cdot 1 / 2$ & $1 / 16$ & $-A \cdot A$ \\
\hline & & $-A$ & $1-$ & 11 & & & - & \\
\hline & & $\mathrm{B}$ & -1 & 11 & $v_{l}(5,2)$ & $1 / 8 \cdot 1 / 2$ & $1 / 16$ & $-A \cdot B$ \\
\hline & & $-B$ & $1-$ & $1-1$ & & & - & \\
\hline & & $\mathrm{C}$ & 1 & $1 \quad-1$ & & & - & \\
\hline & & $-\mathrm{C}$ & $-1-$ & 11 & & & - & \\
\hline & & $\mathrm{D}$ & 1 & $\begin{array}{ll}1 & 1\end{array}$ & & & - & \\
\hline & & $-\mathrm{D}$ & $-1-$ & $1 \quad-1$ & & & - & \\
\hline & & & & & & \multicolumn{3}{|r|}{$-1 / 16(\mathrm{AA}+\mathrm{AB})$} \\
\hline \multirow[t]{8}{*}{$-\mathrm{B}$} & \multirow[t]{8}{*}{$\begin{array}{lll}1 & -1 & -1\end{array}$} & $\mathrm{~A}$ & -1 & $1 \quad-1$ & & & - & \\
\hline & & $-A$ & $1-$ & 11 & & & - & \\
\hline & & $\mathrm{B}$ & -1 & 11 & & & - & \\
\hline & & $-\mathrm{B}$ & $1-$ & $1 \quad-1$ & & & - & \\
\hline & & $\mathrm{C}$ & 1 & $1 \quad-1$ & & & - & \\
\hline & & $-\mathrm{C}$ & $-1-$ & 11 & $v_{1}(6,1)$ & $1 / 8 \cdot 1 / 2$ & $1 / 16$ & $-B \cdot-C$ \\
\hline & & $\mathrm{D}$ & 1 & 11 & & & - & \\
\hline & & $-\mathrm{D}$ & $-1-$ & $1 \quad-1$ & $v_{1}(6,2)$ & $1 / 8 \cdot 1 / 2$ & $1 / 16$ & $-B \cdot-D$ \\
\hline & & & & & & \multicolumn{3}{|r|}{ 1/16(BC+BD) } \\
\hline \multirow[t]{8}{*}{$-\mathrm{C}$} & \multirow[t]{8}{*}{$\begin{array}{lll}-1 & -1 & 1\end{array}$} & $\mathrm{~A}$ & -1 & $1-1$ & $v_{1}(7,1)$ & $1 / 8 \cdot 1 / 2$ & $1 / 16$ & $-C \cdot A$ \\
\hline & & $-A$ & $1-$ & 11 & & & - & \\
\hline & & $\mathrm{B}$ & -1 & 11 & $v_{1}(7,2)$ & $1 / 8 \cdot 1 / 2$ & $1 / 16$ & $-\mathrm{C} \cdot \mathrm{B}$ \\
\hline & & $-\mathrm{B}$ & $1-$ & $1-1$ & & & - & \\
\hline & & $\mathrm{C}$ & 1 & $1 \quad-1$ & & & - & \\
\hline & & $-\mathrm{C}$ & $-1-$ & 11 & & & - & \\
\hline & & $\mathrm{D}$ & 1 & 11 & & & - & \\
\hline & & $-\mathrm{D}$ & $-1-$ & $1 \quad-1$ & & & - & \\
\hline & & & & & & \multicolumn{3}{|r|}{$-1 / 16(C A+C B)$} \\
\hline \multirow[t]{8}{*}{$-\mathrm{D}$} & \multirow[t]{8}{*}{$\begin{array}{lll}-1 & -1 & -1\end{array}$} & $\mathrm{~A}$ & -1 & $1 \quad-1$ & & & - & \\
\hline & & $-A$ & $1-$ & 11 & & & - & \\
\hline & & $\mathrm{B}$ & -1 & 11 & & & - & \\
\hline & & $-B$ & $1-$ & $\begin{array}{ll} & -1 \\
\end{array}$ & & & - & \\
\hline & & $\mathrm{C}$ & 1 & $\begin{array}{ll}1 & -1 \\
\end{array}$ & & & - & \\
\hline & & $-\mathrm{C}$ & $-1-$ & 11 & $v_{1}(8,1)$ & $1 / 8 \cdot 1 / 2$ & $1 / 16$ & $-\mathrm{D} \cdot-\mathrm{C}$ \\
\hline & & $\mathrm{D}$ & 1 & $\begin{array}{ll}1 & 1 \\
\end{array}$ & & & - & \\
\hline & & $-\mathrm{D}$ & $-1-$ & $1 \quad-1$ & $v_{1}(8,2)$ & $1 / 8 \cdot 1 / 2$ & $1 / 16$ & $-\mathrm{D} \cdot-\mathrm{D}$ \\
\hline & & & & & & & & 1/16(DC+DD $)$ \\
\hline
\end{tabular}

Table 2. $R_{l}(\tau)$.

Adding up the terms in the above table, we obtain $R_{l}(\tau)$ :

$$
\begin{aligned}
& R_{1}(\tau)=\frac{1}{16}\left(\begin{array}{l}
-R_{A A}-R_{A B}+R_{B C}+R_{B D}-R_{C A}-R_{C B}+R_{D C}+R_{D D} \\
-R_{A A}-R_{A B}+R_{B C}+R_{B D}-R_{C A}-R_{C B}+R_{D C}+R_{D D}
\end{array}\right) \\
& =\frac{1}{8}\left(-R_{A A}-R_{A B}+R_{B C}+R_{B D}-R_{C A}-R_{C B}+R_{D C}+R_{D D}\right) .
\end{aligned}
$$


C.3. $R_{2}(\tau)$

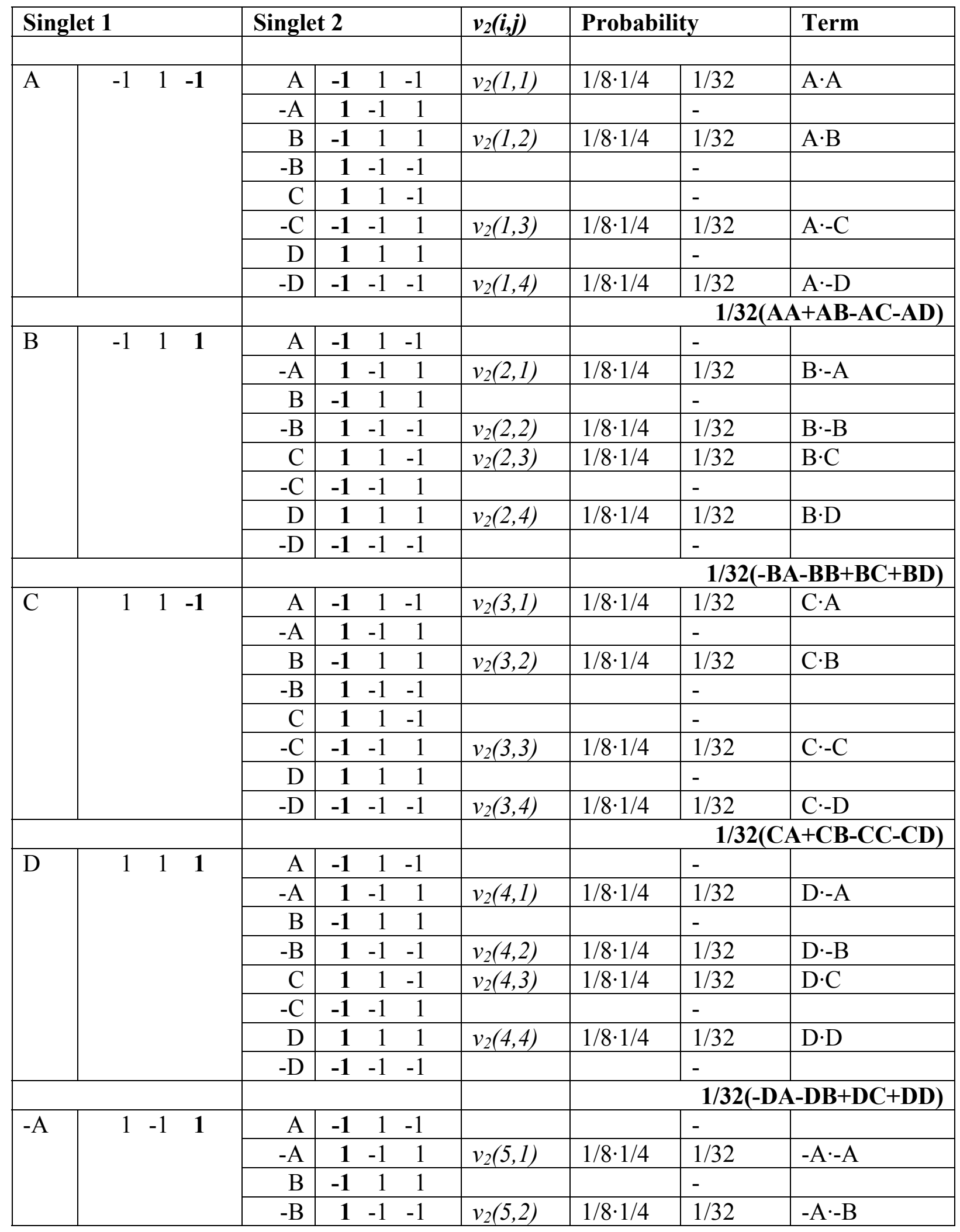




\begin{tabular}{|c|c|c|c|c|c|c|c|}
\hline & & $\mathrm{C}$ & $\begin{array}{lll}1 & 1 & -1 \\
\end{array}$ & $v_{2}(5,3)$ & $1 / 8 \cdot 1 / 4$ & $1 / 32$ & $-A \cdot C$ \\
\hline & & $-\mathrm{C}$ & $\begin{array}{lll}-1 & -1 & 1 \\
\end{array}$ & & & - & \\
\hline & & $\mathrm{D}$ & $\begin{array}{lll}1 & 1 & 1 \\
\end{array}$ & $v_{2}(5,4)$ & $1 / 8 \cdot 1 / 4$ & $1 / 32$ & $-A \cdot D$ \\
\hline & & $-\mathrm{D}$ & $\begin{array}{lll}\mathbf{- 1} & -1 & -1\end{array}$ & & & - & \\
\hline & & & & & \multicolumn{3}{|c|}{ 1/32(AA+AB-AC-AD) } \\
\hline \multirow[t]{8}{*}{$-B$} & \multirow[t]{8}{*}{$\begin{array}{lll}1 & -1 & -1\end{array}$} & $\mathrm{~A}$ & $\begin{array}{lll}\mathbf{- 1} & 1 & -1 \\
\end{array}$ & $v_{2}(6,1)$ & $1 / 8 \cdot 1 / 4$ & $1 / 32$ & $-B \cdot A$ \\
\hline & & $-\mathrm{A}$ & $\begin{array}{lll}1 & -1 & 1 \\
\end{array}$ & & & - & \\
\hline & & $\mathrm{B}$ & $\begin{array}{lll}-1 & 1 & 1 \\
\end{array}$ & $v_{2}(6,2)$ & $1 / 8 \cdot 1 / 4$ & $1 / 32$ & $-B \cdot B$ \\
\hline & & $-\mathrm{B}$ & $\begin{array}{lll}\mathbf{1} & -1 & -1 \\
\end{array}$ & & & - & \\
\hline & & $\mathrm{C}$ & $\begin{array}{lll}1 & 1 & -1 \\
\end{array}$ & & & - & \\
\hline & & $-\mathrm{C}$ & $\begin{array}{lll}-\mathbf{- 1} & -1 & 1 \\
\end{array}$ & $v_{2}(6,3)$ & $1 / 8 \cdot 1 / 4$ & $1 / 32$ & $-B \cdot-C$ \\
\hline & & $\mathrm{D}$ & $\begin{array}{lll}1 & 1 & 1\end{array}$ & & & - & \\
\hline & & $-\mathrm{D}$ & $\begin{array}{lll}-1 & -1 & -1 \\
\end{array}$ & $v_{2}(6,4)$ & $1 / 8 \cdot 1 / 4$ & $1 / 32$ & $-B \cdot-D$ \\
\hline & & & & & \multicolumn{3}{|c|}{ 1/32(-BA-BB+BC+BD) } \\
\hline \multirow[t]{8}{*}{$-\mathrm{C}$} & \multirow{8}{*}{$\begin{array}{lll}-1 & -1 & 1\end{array}$} & A & $\begin{array}{lll}\mathbf{- 1} & 1 & -1 \\
\end{array}$ & & & - & \\
\hline & & $-A$ & $\begin{array}{lll}1 & -1 & 1 \\
\end{array}$ & $v_{2}(7,1)$ & $1 / 8 \cdot 1 / 4$ & $1 / 32$ & $-C \cdot-A$ \\
\hline & & $\mathrm{B}$ & $\begin{array}{lll}-1 & 1 & 1 \\
\end{array}$ & & & - & \\
\hline & & $-\mathrm{B}$ & $\begin{array}{lll}1 & -1 & -1 \\
\end{array}$ & $v_{2}(7,2)$ & $1 / 8 \cdot 1 / 4$ & $1 / 32$ & $-C \cdot-B$ \\
\hline & & $\mathrm{C}$ & $\begin{array}{lll}1 & 1 & -1 \\
\end{array}$ & $v_{2}(7,3)$ & $1 / 8 \cdot 1 / 4$ & $1 / 32$ & $-\mathrm{C} \cdot \mathrm{C}$ \\
\hline & & $-\mathrm{C}$ & $\begin{array}{lll}\mathbf{- 1} & -1 & 1 \\
\end{array}$ & & & - & \\
\hline & & $\mathrm{D}$ & $\begin{array}{lll}1 & 1 & 1 \\
\end{array}$ & $v_{2}(7,4)$ & $1 / 8 \cdot 1 / 4$ & $1 / 32$ & $-C \cdot D$ \\
\hline & & $-\mathrm{D}$ & $\begin{array}{lll}-\mathbf{1} & -1 & -1\end{array}$ & & & - & \\
\hline & & & & & \multicolumn{3}{|c|}{ 1/32(CA+CB-CC-CD) } \\
\hline \multirow[t]{8}{*}{$-\mathrm{D}$} & \multirow[t]{9}{*}{$\begin{array}{lll}-1 & -1 & -1\end{array}$} & A & $\begin{array}{lll}\mathbf{- 1} & 1 & -1 \\
\end{array}$ & $v_{2}(8,1)$ & $1 / 8 \cdot 1 / 4$ & $1 / 32$ & $-D \cdot A$ \\
\hline & & $-\mathrm{A}$ & $\begin{array}{lll}1 & -1 & 1 \\
\end{array}$ & & & - & \\
\hline & & $\mathrm{B}$ & $\begin{array}{lll}\mathbf{- 1} & 1 & 1 \\
\end{array}$ & $v_{2}(8,2)$ & $1 / 8 \cdot 1 / 4$ & $1 / 32$ & $-\mathrm{D} \cdot \mathrm{B}$ \\
\hline & & $-\mathrm{B}$ & $\begin{array}{lll}1 & -1 & -1 \\
\end{array}$ & & & - & \\
\hline & & $\mathrm{C}$ & $\begin{array}{lll}1 & 1 & -1 \\
\end{array}$ & & & - & \\
\hline & & $-\mathrm{C}$ & $\begin{array}{lll}\mathbf{- 1} & -1 & 1 \\
\end{array}$ & $v_{2}(8,3)$ & $1 / 8 \cdot 1 / 4$ & $1 / 32$ & $-\mathrm{D} \cdot-\mathrm{C}$ \\
\hline & & $\mathrm{D}$ & $\begin{array}{lll}\mathbf{1} & 1 & 1 \\
\end{array}$ & & & - & \\
\hline & & $-\mathrm{D}$ & $\begin{array}{lll}-\mathbf{- 1} & -1 & -1 \\
\end{array}$ & $v_{2}(8,4)$ & $1 / 8 \cdot 1 / 4$ & $1 / 32$ & $-\mathrm{D} \cdot-\mathrm{D}$ \\
\hline & & & & & & $1 / 32$ & -DB+DC+DD) \\
\hline
\end{tabular}

Table 3. $R_{2}(\tau)$.

Adding up the terms in the above table, we obtain $R_{2}(\tau)$ :

$$
\begin{gathered}
R_{2}(\tau)=\frac{1}{32}\left(\begin{array}{l}
R_{A A}+R_{A B}-R_{A C}-R_{A D}-R_{B A}-R_{B B}+R_{B C}+R_{B D} \\
+R_{C A}+R_{C B}-R_{C C}-R_{C D}-R_{D A}-R_{D B}+R_{D C}+R_{D D} \\
+R_{A A}+R_{A B}-R_{A C}-R_{A D}-R_{B A}-R_{B B}+R_{B C}+R_{B D} \\
+R_{C A}+R_{C B}-R_{C C}-R_{C D}-R_{D A}-R_{D B}+R_{D C}+R_{D D}
\end{array}\right) \\
=\frac{1}{16}\left(\begin{array}{l}
R_{A A}+R_{A B}-R_{A C}-R_{A D}-R_{B A}-R_{B B}+R_{B C}+R_{B D} \\
+R_{C A}+R_{C B}-R_{C C}-R_{C D}-R_{D A}-R_{D B}+R_{D C}+R_{D D}
\end{array}\right) .
\end{gathered}
$$




\section{References}

[Abidi] A.A. Abidi and R.G. Meyer, "First zero crossing analysis," IEEE J. Solid-State Circuits, vol. 48, no. 6, pp. 794-802, Dec. 1983.

[Amirkhany-1] A. Amirkhany, V. Stojanović and M.A. Horowitz, "Multi-tone signaling for high-speed backplane electrical links," in Proc. GLOBECOM, Dec. 2004, pp. 1111-1117.

[Amirkhany-2] A. Amirkhany, A. Abbasfar, J. Savoj, M. Jeeradit, B. Garlepp, V. Stojanović, and M. Horowitz, "A 24Gb/s Software Programmable Multi-Channel Transmitter," in Symp. VLSI Circuits Dig. Tech. Papers, Jun. 2007, pp. 38-39.

[Arabi] T.R. Arabi, A.T. Murphy, T.K. Sarkar, R.F. Harrington, A.R. Djordjević, "On the Modeling of Conductor and Substrate Losses in Multiconductor, Multidielectric Transmission Line Systems." IEEE Transactions on Microwave Theory and Techniques, vol. 39, no. 7, pp. 1090-1097, Jul. 1991.

[Balan] V. Balan, J. Caroselli, J.-G. Chern, C. Chow et al., "A 4.8-6.4Gbps serial link for backplane applications using decision feedback equalization", IEEE J. Solid-State Circuits, vol. 40, no. 9, pp. 19571967, Sep. 2005.

[vdBeek] R.C.H. van de Beek, C.S. Vaucher, D.M.W. Leenaerts, E.A.M. Klumperink, and B. Nauta, "A 2.5-10-GHz Clock Multiplier

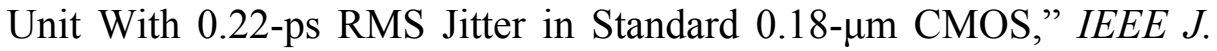
Solid-State Circuits, vol. 39, no. 1, pp. 1862-1872, Nov. 2004.

[Bingham] J.A.C. Bingham, "Multicarrier modulation for data transmission: an idea whose time has come," IEEE Commun. Mag., vol. 28, pp. 5-14, May 1990.

[Bode] H.W. Bode, "Relations between attenuation and phase in feedback amplifier design”, Bell System Tech. J., vol. 19, pp. 421-454, July 1940.

[Brianti] G. Brianti, "Distortion of fast pulses in coaxial cables: numerical analysis and applications," CERN 65-10, 1965. (Available at http://cdsweb.cern.ch.)

[Chipman] R.A. Chipman, Transmission Lines. New York: McGraw-Hill, 1968.

[Choi]

J.-S. Choi, M.-S. Hwang and D.-K. Jeong, “A 0.18- $\mu$ mCMOS 3.5-Gb/s continuous-time adaptive cable equalizer using enhanced low-frequency gain control method," IEEE J. Solid-State Circuits, vol. 39, no. 3, pp. 419-425, Mar. 2004. 
[Couch]

[Curtins]

[Dally-1]

[Dally-2]

[Dally-3]

[Djordjević]

[Eken]

[vEtten]

[Farjad-Rad]

[Gai]

[Gardiol]

[Grivet]

[Guillemin]
L.W. Couch, Digital and analog communication systems, Prentice-Hall, 2001.

H. Curtins, A.V. Shah, "Pulse Behavior of Transmission Lines with Dielectric Losses," IEEE Transactions on Circuits And Systems, vol. Cas-32, no. 8, Aug. 1985.

W.J. Dally and J. Poulton, "Transmitter equalization for $4 \mathrm{~Gb} / \mathrm{s}$ signaling," IEEE Micro, vol. 17, no. 1, pp. 48-56, Jan.-Feb. 1997.

W.J. Dally, M.-J.E. Lee, F.-T. An, J. Poulton and S. Tell, "Highperformance electrical signaling," in Proc. Fifth International Conference on Massively Parallel Processing, Jun. 1998, pp. 11-16.

W.J. Dally and J.W. Poulton, Digital Systems Engineering: Cambridge University Press, 1998.

A.R. Djordjević, R.M. Biljic, V.D. Likar-Smiljanic, T.K. Sarkar, "Wideband Frequency-Domain Characterization of FR-4 and TimeDomain Causality," IEEE Transactions on Electromagnetic Compatibility, vol. 43, no. 4, pp. 662-667, Nov. 2001.

Y.A. Eken and J.P. Uyemura, "A 5.9-GHz Voltage-Controlled Ring

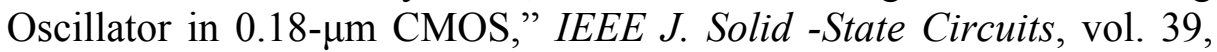
no. 1, pp. 230-233, Jan. 2004.

W.C. van Etten, private communication.

R. Farjad-Rad, C.K. Yang, M. Horowitz, and T. Lee, "A 0.3- $\mu \mathrm{m}$ CMOS $8 \mathrm{~Gb} / \mathrm{s}$ 4-PAM serial link transceiver," IEEE J. Solid-State Circuits, vol. 35 , no. 5, pp. 757-764, May 2000.

W. Gai, Y. Hidaka, Y. Koyanagi, J.H. Jiang, H. Osone, and T. Horie, "A 4-channel $3.125 \mathrm{~Gb} / \mathrm{s} / \mathrm{ch}$ CMOS transceiver with $30 \mathrm{~dB}$ equalization," in Symp. VLSI Circuits Dig. Tech. Papers, Jun. 2004, pp. $138-141$.

F.E. Gardiol, Lossy Transmission Lines. Norwood, MA: Artech House, 1987.

P. Grivet and P.W. Hawkes, The Physics of Transmission Lines at High and Very High Frequencies. New York: Academic Press, 1970.

E.A. Guillemin, Theory of Linear Physical Systems. New York:

Wiley, 1963. 
[Hall]

[Johnson]

[Jonscher]

[Kennedy]

[Kerst]

[Krishna]

[Kudoh]

[Lee]

[Llopart]

[Lucky]

[Maillard]

[McNeill]

[Nam]
S. Hall, T. Liang, H. Heck, and D. Shykind, "Modeling requirements for transmission lines in multi-gigabit systems," IEEE 13th Topical Meeting on Electrical Performance of Electronic Packaging, pp. 67-70, 2004.

H. Johnson and M. Graham, High-Speed Digital Design: A Handbook of Black Magic, Prentice Hall, 1993.

A.K. Jonscher, "Dielectric relaxation in solids," J. Phys. D: Appl. Phys. 32, pp. R57-R70, 1999.

J. Kennedy and R. Eberhart, "Particle swarm optimization," in Proc.

IEEE Int. Conf. on Neural Networks, vol. IV, 1995, pp. 1942-1948.

D.W. Kerst and J.C. Sprott, "Electrical circuit modeling of conductors with skin effect," J. Appl. Phys. 60 (2), 1986.

K. Krishna, D.A. Yokoyama-Martin, A. Caffee, C. Jones, M. Loikkanen, J. Parker et al., "A multigigabit backplane transceiver core in $0.13-\mu \mathrm{m}$ CMOS with a power-efficient equalizer architecture", IEEE J. Solid-State Circuits, vol. 40, no. 12, pp. 2568-2666, Dec. 2005.

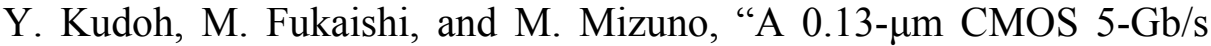
10-m 28 AWG cable transceiver with no-feedback-loop continuoustime post-equalizer," IEEE J. Solid-State Circuits, vol. 38, no. 5, pp. 741-746, May 2003.

M.-J.E. Lee, W.J. Dally, and P. Chiang, "Low-power area-efficient high-speed I/O circuit techniques, "IEEE J. Solid-State Circuits, vol. 35, pp. 1591-1599, Nov. 2000.

X. Llopart and M. Campbell, "First test measurements of a $64 \mathrm{k}$ pixel readout chip working in single photon counting mode," Nuclear Instruments and Methods in Physics Research A, 509, pp. 157-163, 2003.

R.W. Lucky, J. Salz and E.J. Weldon Jr., Principles of Data Communication, McGraw-Hill, 1968.

X. Maillard, Novel circuits and principles for inter-chip communication, PhD. thesis, Vrije Universiteit Brussel, 2005.

J. McNeill, "Jitter in ring oscillators," IEEE J. Solid-State Circuits, vol. 32, no. 6, pp. 870-879, June 1997.

J.-J. Nam and H.-J. Park, "An all-digital CMOS duty cycle correction circuit with a duty-cycle correction range of 15 -to- $85 \%$ for multi-phase applications", IEICE Trans. Electron., vol. E88-C, no. 4, pp. 773777,April 2005. 
[Nielsen]

[Odlyzko]

[Payne]

[vdPlaats]

[Proakis]

[Ramo]

[Razavi]

[Ren]

[Schelkunoff]

[Schinkel-1]

[Schinkel-2]

[Stojanović]

[Stonick]
K. Nielsen, "A review and comparison of PWM methods for analog and digital input switching power amplifiers," in $102^{\text {nd }}$ Convention of the AES, Mar. 1997.

A. Odlyzko, "Internet growth: Myth and reality, use and abuse," $J$. Computer Resource Management, vol. 102, pp. 23-27, 2001.

R. Payne, P. Landman, B. Bhakta, S. Ramaswamy, S. Wu, J.D. Powers et al., "A $6.25-\mathrm{Gb} / \mathrm{s}$ binary transceiver in $0.13-\mu \mathrm{m}$ CMOS for serial data transmission across high loss legacy backplane channels", IEEE $J$. Solid-State Circuits, vol. 40, no. 12, pp. 2646-2656, Dec. 2005.

J. van der Plaats, The coaxial cable in the time and frequency domain, private communication.

J.G. Proakis, Digital Communications, 3rd ed. New York: McGrawHill, 1995.

S. Ramo, J.R. Whinnery, T. van Duzer, Fields and Waves in Communication Electronics. New York: Prentice Hall, 1993.

B. Razavi, Design of analog CMOS integrated circuits, McGraw-Hill, 2001.

J. Ren, H. Lee, Q. Lin, B. Leibowitz, E-H. Chen, D. Oh, F. Lambrecht, V. Stojanović, C.-K. Ken Yang, J. Zerbe, "Precursor ISI Reduction in High-Speed I/O," in Symp. VLSI Circuits Dig. Tech. Papers, Jun. 2007, pp. 134-135.

S.A. Schelkunoff, "The electromagnetic theory of coaxial transmission lines and cylindrical shields," Bell Syst. Tech. J., vol. 13, pp. 532-579, Oct. 1934.

D. Schinkel, E. Mensink, E.A.M. Klumperink, A.J.M. van Tuijl and B. Nauta, "A 3Gb/s/ch Transceiver for RC-limited On-Chip Interconnects," in IEEE Int. Solid-State Circuits Conf. (ISSCC) Dig. Tech. Papers, Feb. 2005, pp. 386-387.

D. Schinkel, E. Mensink, E.A.M. Klumperink, A.J.M. van Tuijl, and B. Nauta, "A 3-Gb/s/ch Transceiver for 10-mm Uninterrupted RCLimited Global On-Chip Interconnects," IEEE J. Solid-State Circuits, vol. 41, no.1, pp. 297-306, Jan. 2006.

V. Stojanović, A. Ho, B. Garlepp, F. Chen et al., "Adaptive equalization and data recovery in a dual mode (2PAM/4) serial link transceiver," in Symp. VLSI Circuits Dig. Tech. Papers, Jun. 2004, pp. 348-351.

J.T. Stonick, G.-Y.Wei, J.L. Sonntag, and D.K. Weinlader, "An adaptive PAM-4 5-Gb/s backplane transceiver in $0.25 \mu \mathrm{m}$ CMOS," IEEE J. Solid-State Circuits, vol. 38, no. 3, pp. 436-443, Mar. 2003. 
[Svensson]

[Weinstein]

[Weldon]

[Westergaard]

[Zerbe]

[Zogakis]
C. Svensson and G.E. Dermer, Time Domain Modeling of Lossy Interconnects, IEEE Transactions on Advanced Packaging, Vol. 24, No. 2, May 2001

S.B. Weinstein and P.M. Ebert, "Data Transmission by FrequencyDivision Multiplexing Using the Discrete Fourier Transform," IEEE Trans. Communication Technology, vol. COM-19, no.5, pp.628-634, Oct. 1971.

J.A. Weldon et al., "A 1.75-GHz highly integrated narrow-band CMOS transmitter with harmonic-rejection mixers," IEEE J. Solid-State Circuits, vol. 36, pp. 2003-2015, Dec. 2001.

P. Westergaard, T.O. Dickson, and S.P. Voinigescu, "A 1.5V 20/30 $\mathrm{Gb} / \mathrm{s}$ CMOS backplane driver with digital pre-emphasis," in Proc. IEEE Custom Integrated Circuits Conf. (CICC), Oct. 2004, pp. 23-26.

J.L. Zerbe, C.W. Werner, V. Stojanović, F. Chen et al., "Equalization and clock recovery for a $2.5-10-\mathrm{Gb} / \mathrm{s}$ 2-PAM/4-PAM backplane transceiver cell," IEEE J. Solid-State Circuits, vol. 38, no. 12, pp. 2121-2130, Sep. 2003.

T.N. Zogakis and J.M. Cioffi, "The effect of timing jitter on the performance of a discrete multitone system," IEEE Trans. Commun., vol. 44, pp. 799-808, July 1996. 


\section{Samenvatting}

$\mathrm{Er}$ is een grens aan de snelheid waarmee (digitale) gegevens in de vorm van elektrische stromen via koperkabels en printplaten kunnen worden verzonden. Het zogenaamde huideffect en de diëlectrische verliezen veroorzaken signaaldemping en -dispersie, die de maximaal haalbare transmissiesnelheid beperken. Tevens kunnen er signaalreflecties optreden als er discontinuïteiten zitten in de elektrische impedantie. Als gevolg van al deze effecten ontstaat er intersymbool-interferentie: naburige symbolen interfereren met elkaar.

Het doel van dit promotieonderzoek is het vinden van nieuwe egalisatie- en modulatietechnieken die kunnen worden ingezet om de maximaal haalbare transmissiesnelheid over koperkanalen te verhogen. Voorwaarde daarbij is wel dat foutenkans (dat symbolen incorrect worden ontvangen) niet omhoog gaat. De nieuwe technieken moeten tevens goed aansluiten bij toekomstige generaties van technologieën voor het maken van geïntegreerde circuits (ICs).

In Hoofdstuk 2 wordt een praktisch simulatiemodel besproken voor koperkabels en printplaten. Dit model kan gebruikt worden voor tijddomeinsimulaties met een hoge tijdsresolutie. Zowel het huideffect als de diëlectrische verliezen worden gemodelleerd. Een causale impulsresponsie wordt gegarandeerd doordat de complexe impedanties van het huideffect en van de diëlectrische verliezen voldoen aan de Kramers-Kronig relaties. De juiste modelparameters worden gevonden door de modelvoorspelling gelijk te stellen aan de resultaten van metingen aan drie coaxiale koperkabels, een gevlochten aderpaar en een koperspoor op een printplaat.

In Hoofdstuk 3 wordt een analoge implementatie van een OFDM-systeem ('Orthogonal Frequency Division Multiplexing') beschreven. Deze analoge implementatie is gebaseerd op analoge vermenigvuldigers en integreer-schakelingen. Zo'n systeem is gevoelig voor onnauwkeurigheden in de synchronisatie, zoals fluctuaties in de signaalfase ('jitter') en afwijkingen in de duty-cycle. De invloed hiervan wordt geanalyseerd. De bevindingen zijn:

- Jitter veroorzaakt overspraak tussen het in-fasekanaal en het kwadratuurkanaal in een gegeven OFDM-subband. Gegeven een zekere RMS-variantie in de jitter kunnen, voor gelijke bitfoutenkans, op laagfrequente subbanden meer bits gemoduleerd worden dan op hoogfrequente subbanden. De oorzaak hiervan is dat eenzelfde tijdsverschuiving een grotere fasefout geeft bij hogere frequenties.

- $\quad$ Onnauwkeurigheden in de duty-cycle verzoorzaken overspraak tussen subbanden op verschillende frequenties omdat de OFDM-orthogonaliteit verloren gaat.

- Harmonischen ten gevolge van de schakelende vermenigvuldigers maken het gebruik van veel subbanden onmogelijk door spectrale overlap.

De conclusie is dat dit analoge OFDM-systeem, door de hoge gevoeligheid voor onnauwkeurigheden in de synchronisatie, momenteel niet haalbaar is en dat traditionele PAM systemen beter haalbaar zijn (en eenvoudiger te implementeren). De laatste genieten dus de voorkeur voor koperkanalen met een monotoon afnemende overdrachtsfunctie, zonder diepe nulpunten in de frequentieoverdrachtsfunctie.

In Hoofdstuk 4 wordt beschreven hoe een egalisatieschakeling voor koperkabels en printplaten gemaakt kan worden door de zender te laten schakelen tussen slechts twee 
spanningen. In het geval dat een ' 1 '-bit verstuurd moet worden, wordt gedurende één bittijd eerst een positieve spanning op de lijn gezet en vervolgens een negatieve spanning. Het omgekeerde wordt gedaan wanneer een '0'-bit moet worden verzonden. Door het instellen van de verhouding tussen de positieve en negatieve spanning (de duty-cycle), kan het filter aangepast worden aan het kanaal. Dit zogenaamde 'pulsbreedtemodulatiefilter' (PWM-filter) heeft dus slechts één knop (instelparameter) nodig.

Deze techniek is een alternatief voor de veel toegepaste FIR-filters (lineaire filters met een eindige impulsresponsie). De PWM-filtertechniek doet zijn voordeel met de tijdsnauwkeurigheid die inherent aanwezig is in nieuwe technologieën voor geïntegreerde schakelingen. We zien steeds hogere schakelsnelheden (en een steeds hogere tijdsnauwkeurigheid). FIR-filters echter vereisen een meer nauwkeurige amplitude, iets dat steeds lastiger wordt in de nieuwe technologieën. Met elke technologiegeneratie wordt namelijk de spanningsruimte kleiner en neemt de relatieve amplitudenauwkeurigheid af.

De overdrachtsfunctie van het PWM-filter past zeer goed op die van de koperkanalen. Het PWM-filter past beter op de kanalen dan een 2-taps FIR-filter, zowel één met een tapafstand van een hele symbooltijd (SSF) als één met een tapafstand van een halve symbooltijd. Het PWM-filter kan gezien worden als een filter van een hogere orde met slechts één instelparameter. Tijddomeinsimulaties van een theoretisch kanaal met uitsluitend huideffect, laten zien dat ongeveer tweemaal de transmissiesnelheid behaald kan worden als met een 2tap SSF filter, bij gelijke piekdistortie.

Aan de uitgang van het kanaal is een normaal pulsamplitudegemoduleerd (PAM) signaal beschikbaar. Uit berekeningen blijkt dat de oogopening aan de ontvangstkant hetzelfde is als die bij een 2-taps FIR-filter (aangenomen dat zowel het FIR-filter als het PWM-filter dezelfde spanningsruimte kunnen benutten). De ontvangstcircuits kunnen dus ongewijzigd blijven.

Twee $0.13 \mu \mathrm{m}$ CMOS-chips en een 90nm CMOS-chip zijn gemaakt om het PWM-filter te testen. Metingen met deze prototypes laten het volgende zien: een succesvolle ontvangst van de gegevens is mogelijk met een bitfoutenkans (BER) van $10^{-12}$. Dit is gemeten over coaxiale en gevlochten-paar koperkabels, met tot $30 \mathrm{~dB}$ verlies op de Nyquist frequentie. Deze gemeten verliescompensatie is aanzienlijk hoger dan de maximaal haalbare $20 \mathrm{~dB}$ voor een 2 -tap SSFfilter (en $30 \mathrm{~dB}$ voor meer complexe egalisatiecircuits met meer instelparameters). Een bitsnelheid van $5 \mathrm{~Gb} / \mathrm{s}$ werd bereikt op alle kanalen. De verschillende kabels hebben elk een andere verhouding van huideffect-verlies t.o.v. diëlectrisch verlies, en alle kanalen hebben $30 \mathrm{~dB}$ totaal verlies (beide effecten tezamen) op een frequentie van $2.5 \mathrm{GHz}$. Op een FR4printspoor werd $25 \mathrm{~dB}$ aan verliescompensatie behaald.

In Hoofdstuk 5 wordt besproken hoe de PWM-filtertechniek kan worden uitgebreid naar een versie met meerdere filtertaps. De duty-cycle van dit multitap PWM-filter is een functie van meerdere bits. Zo kunnen meer complexe overdrachtsfuncties gemaakt worden, zoals dat ook kan met multitap FIR-filters. De zender hoeft nog steeds slechts te schakelen tussen twee spanningen, zodat het weer mogelijk is om een implementatie te maken die gebruik maakt van de hoge tijdsresolutie die beschikbaar is in de nieuwe generaties CMOS-technologieën. Om de juiste timing van de schakelmomenten te vinden, wordt de methode van het gelijke oppervlak gebruikt. M.b.v. tijddomein simulaties wordt geillustreerd dat de oogopeningen vrijwel gelijk zijn aan die bij het equivalente 3-taps SSF filter. 
De vermogensspectraaldichtheidsfuncties (PSD-functies) van de 3-taps PWM-filters laten bij frequenties rondom de Nyquist frequentie een hogere vermogensdichtheid zien dan die van het FIR-filter. De aanwezigheid van harmonischen is inherent aan PWM. Een systeemontwerper zal deze bijwerking van PWM moeten afwegen tegen het eerder genoemde voordeel van het gebruik van een egalisatietechniek waarbij de zender slechts hoeft te schakelen tussen twee spanningen. Zolang het kanaal het karakter heeft van een laagdoorlaatfilter, zal het hoogfrequente gedeelte van het spectrum uitgefilterd worden. 


\section{List of publications}

[Furtado] H. Furtado, J. Schrader, A. Marchioro and P. Moreira, "Delay 25 an ASIC for timing adjustment in LHC," 11th Workshop on Electronics for LHC and future Experiments, Heidelberg, Sep. 2005. (Available at http://hc-workshop2005.web.cern.ch/lhc-workshop-2005/ParallelSessionsA/24-HugoFurtado.pdf.)

[Schrader-1] J.H.R. Schrader, E.A.M. Klumperink, J.L. Visschers and B. Nauta, "Data communication in read-out systems: how fast can we go over copper wires?," Nuclear Instruments and Methods in Physics Research Section A, vol. 531, no. 1-2, pp. 221-227, Sep. 2004.

[Schrader-2] J.H.R. Schrader, E.A.M. Klumperink, J.L. Visschers and B. Nauta, "Jitter Limitations on a Gigabit Copper Multi-Carrier System," in Proc. 12th ProRISC workshop on Circuits, Systems and Signal Processing, Nov. 2004, pp. 594-598.

[Schrader-3] J.H.R. Schrader, E.A.M. Klumperink, J.L. Visschers and B. Nauta, "Jitter limitations on multicarrier modulation," in Int. Symp. Circuits and Systems, Jun. 2005, pp. 6090-6093.

[Schrader-4] J.H.R. Schrader, E.A.M. Klumperink, J.L. Visschers and B. Nauta, "CMOS transmitter using pulse-width modulation pre-emphasis achieving $33 \mathrm{~dB}$ loss compensation at 5-Gb/s," in Symp. VLSI Circuits Dig. Tech. Papers, Jun. 2005, pp. 388-391.

[Schrader-5] J.H.R. Schrader, E.A.M. Klumperink, J.L. Visschers and B. Nauta, Equalization of Skin Effect Loss Dominated Channels using Pulse-Width Modulation Pre-Emphasis," in Proc. 12th ProRISC workshop on Circuits, Systems and Signal Processing, Nov. 2005, pp. 284-288.

[Schrader-6] J.-R. Schrader, E.A.M. Klumperink, J.L. Visschers and B. Nauta, "Pulse-Width Modulation Pre-Emphasis Applied in a Wireline Transmitter, Achieving $33 \mathrm{~dB}$ Loss Compensation at $5-\mathrm{Gb} / \mathrm{s}$ in $0.13-\mu \mathrm{m}$ CMOS," IEEE J. Solid-State Circuits, vol. 41, no. 4, pp. 990-999, Apr. 2006.

[Schrader-7] J.H.R. Schrader, E.A.M. Klumperink, J.L. Visschers and B. Nauta, "Wireline equalization using pulse-width modulation," in Proc. IEEE Custom Integrated Circuits Conf. (CICC), Sep. 2006, pp. 591-598. 


\section{Dankwoord}

Een proefschrift schrijf je niet alleen. Met de steun van heel veel vrienden en collega's is het tot stand gekomen. Bij deze wil ik een aantal mensen bedanken met wie ik met veel plezier heb samengewerkt de afgelopen jaren.

Ik dank mijn promotor prof. Bram Nauta voor zijn motivatie en zijn openheid van geest. Ook dank ik hem voor het creëren van de juiste randvoorwaarden voor mijn promotietraject. Bij de leerstoel ICD heb ik een bijzonder mooie tijd gehad. De fantastische belevenissen rondom een zeker bedrijf in Silicon Valley zullen me lang bijblijven. En: wanneer veranderen ze nou eens de naam 'Fir ballroom' in 'PWM ballroom'?

Eric Klumperink ben ik buitengewoon dankbaar voor het aanscherpen van mijn gedachten, zowel bij het vele overleg als voor wat betreft de talloze conceptversies van papers en van het proefschrift die hij van commentaar voorzag. Ik ben ervan overtuigd dat de kwaliteit van mijn schrijfwerk er dankzij Eric enorm op vooruit is gegaan.

Jan Visschers van het NIKHEF wil ik bedanken voor het initiëren van het promotieproject, en voor het organiseren van deelname in de IBM $0,13 \mu \mathrm{m}$ tape-out, precies op het juiste moment. Hij vertrouwde erop dat ik wel iets goeds op de chip zou zetten. Ook organiseerde hij mijn periode aan het CERN, waar ik met veel plezier heb gewerkt en vele leuke en interessante mensen heb ontmoet.

Prof. Van Etten heeft belangrijke bijdragen geleverd op het gebied van de communicatietheorie.

Aan Henk 'imponator' de Vries, Gerard Wienk, Frederik Reenders, Gerdien Lammers, Anne-Johan Annema en Ronan van der Zee wil ik bij deze ook mijn dank betuigen.

Ik herinner me vele interessante en gezellige (ook vaak off-topic) discussies bij de koffie met de collega-promovendi van ICD. Met Daniël Schinkel had ik nuttige gedachtenwisselingen bij het whiteboard. Simon 'potje' Louwsma, mijn kamergenoot, en Vincent Arkesteijn bedank ik voor een bijzonder leuke en gezellige tijd.

Rondom de tape-out data was ik veel op het CERN. Na een hele dag lay-outen gaat er niets boven een glaasje wijn en een stukje brie, op het CERN terras met uitzicht op de Mont-Blanc. Het was fijn om met de DelayChip een kleine bijdrage te kunnen leveren aan de Large Hadron Collider. Bij deze bedank ik David San Segundo Bello, Joop Rövekamp, Ruud Kluit, Vladimir Gromov, Erik Heijne, Bert van Koningsveld, Xavier Llopart, Rafael Ballabriga Sune, Lukas Tlustos en Paulo Moreira.

De studenten Michiel van Elzakker, Chen-Hai Li, Gaurav Nucche Pradhan en Gerrit Ledderhof, wil ik bedanken voor hun werk in het kader van mijn onderzoek.

Bijzonder belangrijk zijn mijn familie en mijn vrienden. Ik ben mijn ouders zeer dankbaar voor de motivatie die ze me hebben gegeven, en voor alle steun en hulp. Ook bedank ik mijn zussen Willemien en Frederieke en tante Tineke. Met mijn vrienden Michiel Brederveld en Gert-Jan Bakker kon ik altijd mijn ervaringen delen.

Tot slot wil ik Dolly bedanken, die lief en leed met me heeft gedeeld en die voor mij steeds een grote steun is geweest, net zoals mijn kinderen Jeroen en Suzanne. Jullie drieën geven me elke dag veel liefde en plezier, en doen me beseffen waar het in het leven om draait. Er is meer in het leven dan alleen het schrijven van een proefschrift...

Jan-Rutger Schrader. 


\section{About the author}

Jan-Rutger Schrader was born on September 27, 1976, in Enkhuizen, The Netherlands. He received the M.S. degree in Electrical and Electronics Engineering from the University of Twente, Enschede, The Netherlands in 2001. For the past five years he has been working toward the Ph.D. degree in the IC Design Group of the CTIT Research Institute and the Department of Electrical Engineering, Mathematics and Computer Science at the same University. His research interests include high speed data-communication and mixed-mode CMOS circuits.

(http://www.schrader-ic.com) 


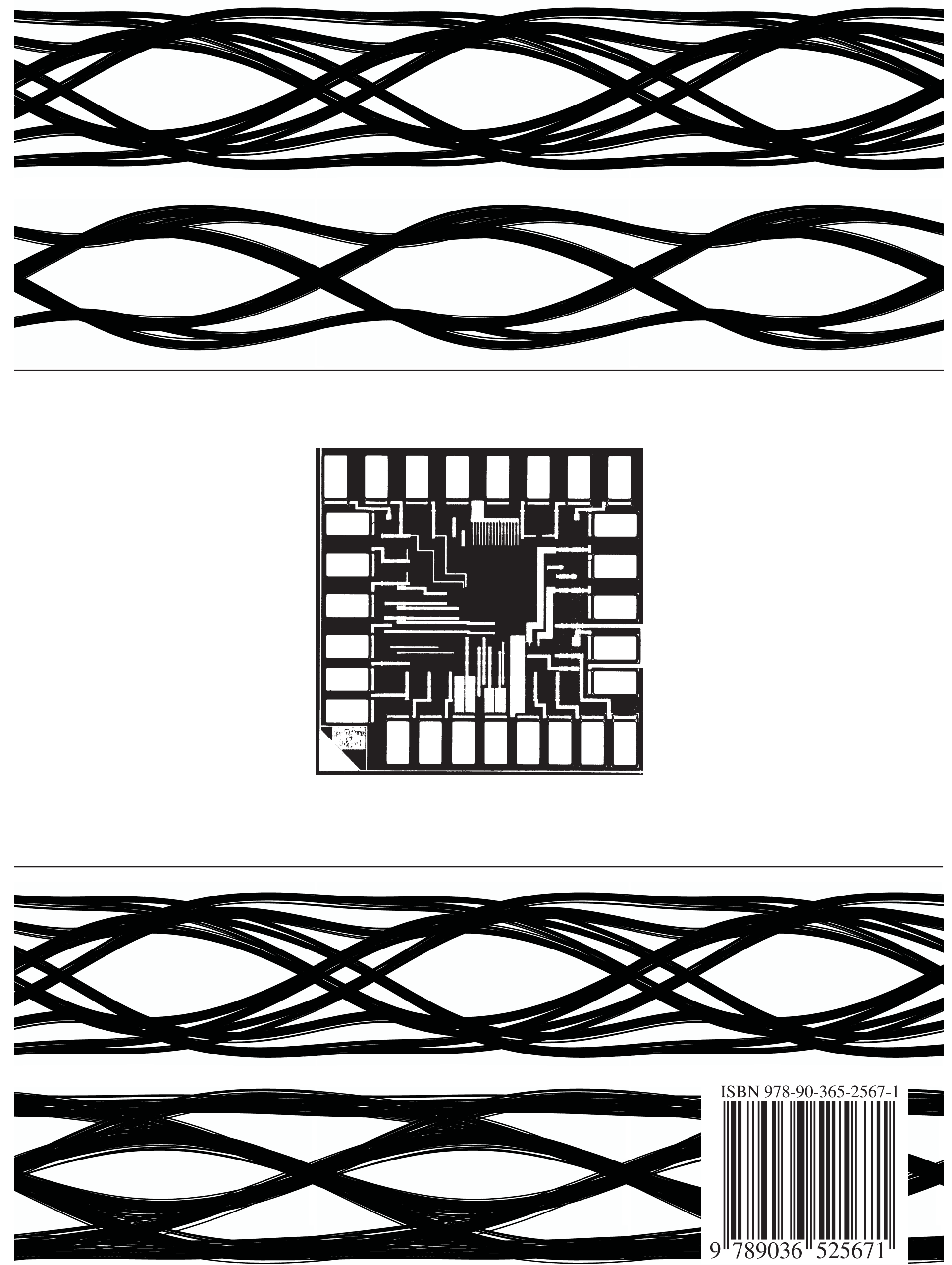